\title{
Receptomics, design of a microfluidic receptor screening technology
}




\section{Thesis committee}

\section{Promotors}

Prof. Dr Robert D. Hall

Special professor Plant Metabolomics

Wageningen University \& Research

Prof. Dr Renger F. Witkamp

Professor of Nutritional Biology and Health

Wageningen University \& Research

\section{Co-promotor}

Dr Maarten A. Jongsma

Senior Scientist, Business Unit Bioscience

Wageningen University \& Research

\section{Other members}

Prof. Dr Jean-Paul Vincken, Wageningen University \& Research

Prof. Dr Sander Kersten, Wageningen University \& Research

Dr Maik Behrens, Technical University of Munich, Germany

Dr Jakob Ley, Symrise AG, Germany

This research was conducted under the auspices of the Graduate School VLAG (Advanced studies in Food Technology, Agrobiotechnology, Nutrition and Health sciences) 


\title{
Receptomics, design of a microfluidic receptor screening technology
}

\author{
Margriet Roelse
}

Thesis

submitted in fulfilment of the requirements for the degree of doctor

at Wageningen University

by the authority of the Rector Magnificus,

Prof. Dr A.P.J. Mol

in the presence of the

Thesis Committee appointed by the Academic Board

to be defended in public

on Tuesday 29 October 2019

at 4 p.m. in the Aula. 


\section{Margriet Roelse}

Receptomics, design of a microfluidic receptor screening technology,

194 pages

PhD thesis, Wageningen University, Wageningen, the Netherlands (2019)

With references, with summary in English

ISBN: 978-94-6395-081-7

DOI: https://doi.org/10.18174/498013 


\section{Table of contents}

Chapter 1 Introduction

Chapter 2 A generic microfluidic biosensor of GPCR-activation

- monitoring cytoplasmic $\mathrm{Ca}^{2+}$ flux in human HEK293 cells

Chapter 3 Metabolomics meets functional assays: coupling LC-MS and microfluidic cell-based receptor-ligand analyses

Chapter 4 Calcium imaging of GPCR activation using arrays of reverse transfected HEK293 cells in a microfluidic system

Chapter 5 The effect of calcium buffering and calcium sensor type on the sensitivity of an array-based bitter receptor screening assay

Chapter 6 Statistical models discriminating between complex samples measured with microfluidic receptor-cell arrays

References

Summary

Nederlandse samenvatting

Abbreviations

Acknowledgements

About the author

List of publications 188

Overview of completed training activities 

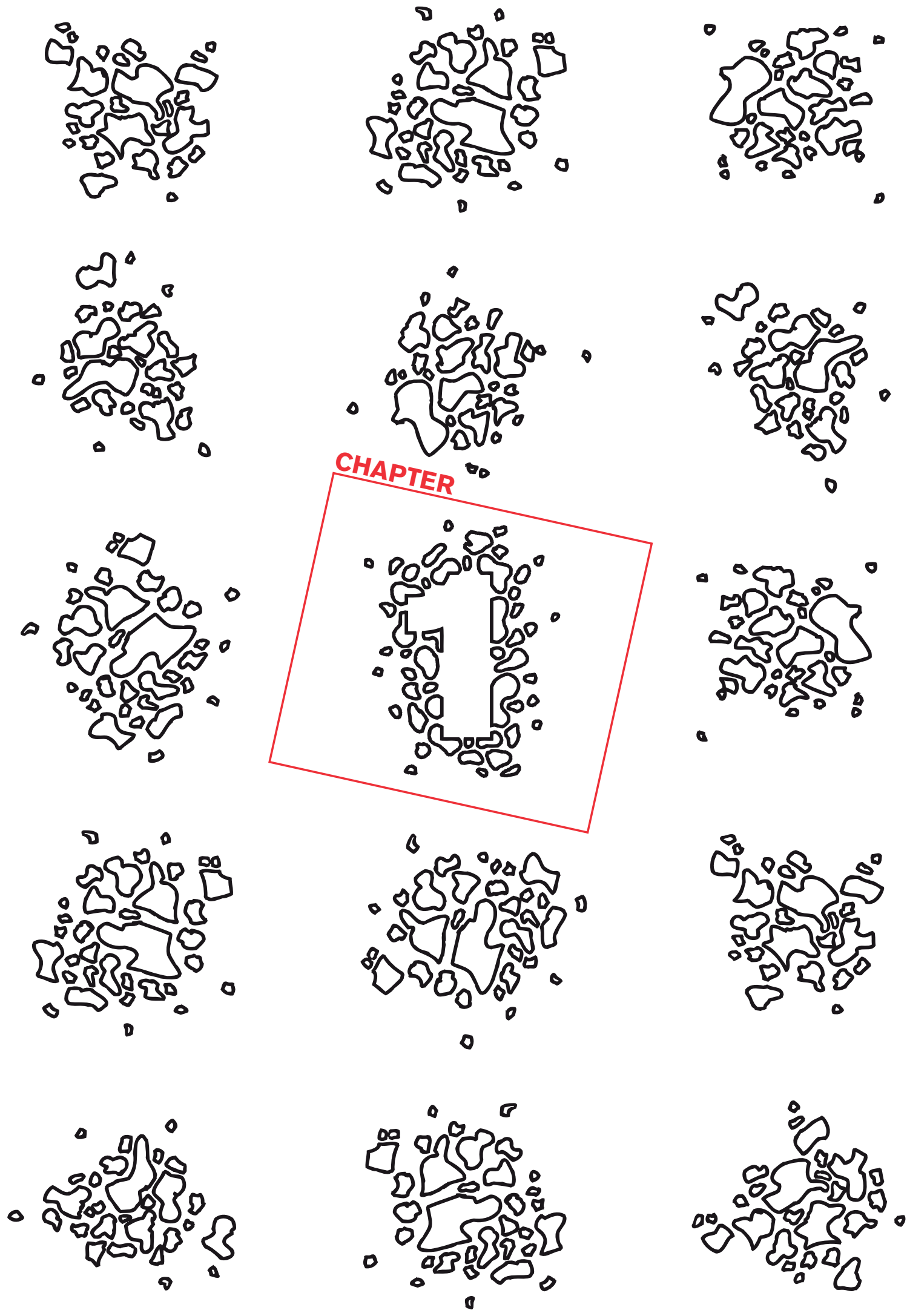


\section{Introduction}




\section{G protein-coupled receptors}

Eukaryotes - including animals, plants, fungi, and protozoa - rely on specialised receptor molecules to receive and to process information both from their environment and their internal milieu. In terms of their modes of action and molecular structures, receptors represent a diverse group of cellular components. Probably the largest structural class in the animal kingdom is comprised of the $\mathrm{G}$ protein coupled receptors, or GPCRs. These proteins, embedded in cellular membranes, including those inside the cell, are activated by a large variety of molecules and other signals such as hormones, pheromones, amino-acids, peptides, proteins, lipids, odorants, drugs, sugars, nucleotides and photons. Activation of GPCRs triggers diverse signal transduction pathways that lead in turn, to cellular responses such as changes in enzyme activity, hormone or neurotransmitter secretion, cell migration or changes in membrane potential of nerve cells to elicit a sense of taste or smell.

With an estimated size of about 800 different members, the GPCR gene family is one of the largest in the human genome [1]. The GPCRs can be divided into 6 classes based on structure and sequence similarity $[2,3]$ as shown in Table 1.

\section{Table 1, GPCR classes}

\begin{tabular}{lll}
\hline Class & Type & Features \\
\hline Class A & Rhodopsin-like & $\begin{array}{l}\text { Comprises ca. 80\% of human GPCRs including Rhodopsin (light), } \\
\text { olfactory and taste receptors }\end{array}$ \\
Class B & Secretin receptor family & $\begin{array}{l}\text { Large N-terminal extracellular domain } \\
\text { [4], ligands are typically peptides or hormones }\end{array}$ \\
Class C & $\begin{array}{l}\text { Metabotropic glutamate/ } \\
\text { pheromone }\end{array}$ & $\begin{array}{l}\text { Receptor homo- or heterodimers, Venus Flytrap (VFT) domain, and } \\
\text { cysteine-rich hinge. Includes sweet and umami receptors [5] }\end{array}$ \\
Class D & $\begin{array}{l}\text { Fungal mating } \\
\text { pheromone receptors }\end{array}$ & $\begin{array}{l}\text { Two subfamilies of yeast GPCRs; STE2 and STE3 involved in sensing } \\
\text { mating pheromones of opposite yeast mating haploid types [6] }\end{array}$ \\
Class E Cyclic AMP receptors & $\begin{array}{l}\text { GPCR type specifically expressed on Dictyostelium discoideum cell } \\
\text { surface [7] }\end{array}$ \\
& & $\begin{array}{l}\text { Frizzled GPCR mediates Wnt binding [8] and the smoothened GPCR } \\
\text { mediates hedgehog signalling [9] }\end{array}$ \\
\hline
\end{tabular}

Even though this family of proteins is large and diverse, its basic structure has been well preserved over the course of evolution [10]. The receptor proteins are embedded in the cell membrane. Like stitches, the amino-acid string swings up and down seven times through the lipid bilayer. For the majority of GPCRs, the N-terminus (or start) of the protein is oriented to the outside or luminal side of the cell membrane and the C-terminus tail is oriented to the inside or cytoplasmic side. There are three extracellular loops and three intracellular loops, each with unique properties and functions [11] (see Figure 1). Meanwhile, the 
complete 3D structure of some GPCRs have been elucidated providing insight into the molecular mechanisms of ligand binding. This information can be used for computational and structure-based discovery of new ligands [12].

\section{Ligand-gated ion channels}

Besides GPCRs, there are also ion channels which can be activated by the binding of specific ligands. Binding of a ligand to these ion channels changes the ion channel conformation allowing the passage of ions into the cell which has a direct effect on the cells polarization [13-15]. In Chapter 3, we used an example of this receptor type as a model, the transient receptor potential channel vanilloid 1 (TRPV1) ion channel. TRPV1 is the receptor for capsaicin, a pungent compound found in chili peppers. The receptor is however, a promiscuous cation channel, with a high preference for calcium, that can be activated by low $\mathrm{pH}$ as well as by physical factors such as heat $\left(>42^{\circ} \mathrm{C}\right)$ and membrane depolarization. More background information is provided in Chapter 3.

\section{GPCRs involved in taste perception}

Taste receptors, located in 'onion' shaped taste buds within the tongue tissue, are specialized receptors for delivering the sensation of taste. Less known but equally important, the same receptors are also expressed in many non-buccal tissues, and are likely to be involved in a variety of physiological functions not related to taste [16].

Molecules from food or other substances which enter through the mouth and can dissolve in the saliva are screened by the taste receptors and their responses are subsequently analyzed and interpreted by the brain. There are 5 distinct taste sensations which can be discriminated by the tongue; sweet, salty, bitter, sour and umami, the latter also known as protein or delicious taste [17]. There is one more controversial taste sensation, the taste of fat or fatty acids $[18,19]$, but evidence is not conclusive.

Salty and sour tastes are sensed by sodium ion channels and proton ion channels respectively [20-22]. The sweet and umami tastes are recognized by the class $C$ receptors that form dimers of two GPCRs [23]. The heterodimer of TAS1R2 and TAS1R3 mediates the sweet sense from sugars as well as non-caloric sweeteners [23-25]. While some studies [26] suggest that T1R3 alone may form a homodimer which senses sweeteners, the evidence is scarce and seems limited to specialized cells.

The heterodimer of TAS1R1 and TAS1R3 mediates the umami taste [23]. The umami receptor is activated by L-Glutamate as well as some other amino acids and can be enhanced by allosteric modulators like inosine-5'-monophosphate (IMP) or guanosine-5'monophosphate (GMP) $[27,28]$. This synergistic effect of IMP and GMP to mono sodium glutamate (MSG) is known to enhance the umami receptor activation by binding to a secondary binding site in TAS1R1 and stabilizing the receptor-ligand interaction. Similar allosteric modes of action have been suggested for the sweet receptor [29, 30] and may also apply to other type C GPCRs. Next to the TAS1R1/TAS1R3 umami receptor there are 
other class C receptors involved in L-Glutamate sensing; the mGluR1 and mGLuR4 receptors (so-called metabotropic glutamine receptors) can sense glutamate, but these are not linked to taste physiology. Glutamate also functions as a neurotransmitter, which is reflected by the predominant distribution of mGluRs in both brain and neurological tissues [31].

Bitter taste is recognized by the TAS2R receptor family of which 25 GPCRs have been putatively identified in humans [32]. The ability to taste bitter substances is considered as an evolutionarily-developed mechanism that allows mammals to recognize toxins in plants. Since these toxins are structurally very diverse, a wide range of bitter receptors have evolved to cover different classes of compounds. These bitter taste receptors have evolved into either broadly-tuned ones or more-specialized ones. Three TAS2R members (TAS2R10, -R14 and -R46) have a broad spectrum of agonists while TAS2R members R3, R5, R8, R13, R49, R50 and R9 are activated by only a few agonists known so far. While most bitter receptors have been de-orphanised, meaning that at least one agonist has been found, there are still a few orphan bitter receptors with unknown ligands, in particular TAS2R19, $-R 42,-R 45$, and $-R 60$. Bitter taste receptors are activated by compounds in concentrations ranging from nanomolar to millimolar [33]. The sensitivity of the bitter taste receptors is generally in accordance with the toxicity of their ligands; the more toxic compounds are therefore sensed at lower concentrations [34].

Apart from taste receptor cells in the tongue, researchers have found taste GPCRs in many other tissues. These include, but are not limited to the respiratory epithelia, gastrointestinal tissue, reproductive organs and the brain [16, 35-37]. The function of sweet taste receptors in the gut probably involves the control of blood sugar levels in a glucose-dependent manner. Activation of gut sweet receptors triggers the release of the Glucagon-like peptide 1 (GLP-1) hormone which, in turn, stimulates the release of insulin from the pancreas. Insulin then decreases blood sugar levels [38]. Bitter receptor expression in the nasal cavity is involved in detecting irritants and bacterial infections in order to evoke an inflammatory response from the upper respiratory innate immune system [39]. The observation that taste receptors not only function in taste cells, but also as sensors linked to various functions of the human physiology makes them interesting targets to study [40].

\section{GPCR signal transduction}

The G protein coupled receptor signaling, or signal transduction, starts with the interaction of an agonist with the extracellular domains of the receptor. The ligand fits between the extracellular loops like a key in a lock and stabilizes the receptor into an active conformation (step one in Figure 1) [41]. Some receptors are specifically tuned and only certain types of molecules fit the uniquely shaped binding pocket. In contrast, some GPCRs are less specific and can interact with many different molecules. Just like turning a key that sets the internal mechanism of the lock in motion, the interaction with the ligand causes conformational changes of the receptor protein. The extracellular loops change position to fit the newly bound molecule. The motion is carried through the transmembrane domains and, in turn, 


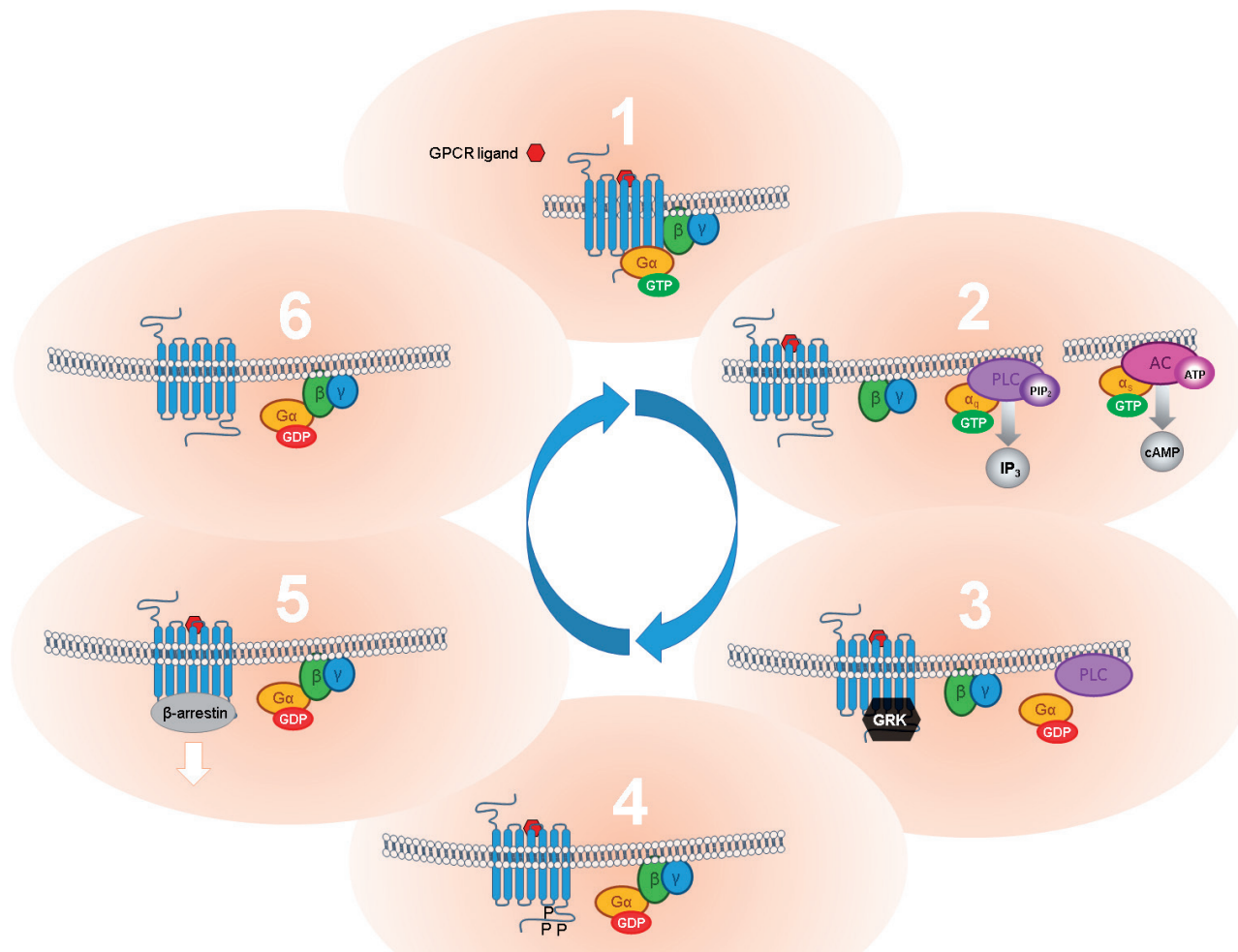

Figure 1, Canonical GPCR signal transduction for Gaq and Gas. Step 1 shows ligand binding and receptor activation. In the G $\alpha$-protein, GDP is exchanged to GTP. Step 2 shows G protein dissociation and interaction of the $\mathrm{G} \alpha$ subunit with an effector component of its signal transduction pathway. In this example the $G \alpha_{q}$ protein interacts with PLC which converts $\mathrm{PIP}_{2}$ into $\mathrm{IP}_{3}$, and the $G \alpha_{s}$ protein interacts with AC which converts ATP into CAMP, see paragraph G $\alpha$ pathways. Step 3 shows the hydrolysis of GTP back to GDP on the Ga protein and GRK interaction with the receptor. Step 4 shows the phosphorylated receptor and the reformation of the $G$ protein complex. Step 5 shows the recruitment of the receptor by $\beta$-arrestin leading to internalization of the receptor. Step 6 shows the receptor after recycling back to the plasma membrane.

affects the intracellular loops to change their conformation. These conformational changes lead to a more stable or "closed" receptor conformation which is called the activated state. This new conformation of the intracellular loops allows a $\mathrm{G}$ protein complex to interact with the intracellular part of the GPCR.

Inside the cell, $\mathrm{G}$ protein complexes are either docked at the intracellular side of the receptor or reside attached to the cell membrane in close proximity of the GPCR. A G protein complex is composed of three regions; alpha, beta and gamma. The $\mathrm{G} \alpha$ is the leading part because this protein interacts most with the intracellular side of the receptor and initiates the next step in the signal transduction. 
The $\mathrm{G} \alpha$ protein carries a guanosine diphosphate (GDP) molecule. When the Ga protein interacts with the receptor, the $\mathrm{G} \alpha$ protein reshapes to take up one inorganic phosphate allowing the conversion of GDP into guanosine triphosphate (GTP). Now the whole G protein complex is affected because as a consequence of the GDP to GTP exchange, the G

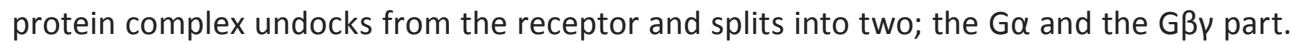
Both parts are now free to play their roles in the further transduction of the intracellular signal (step two in Figure 1). The fate of the signal depends on the $G$ protein type as shown in Table 3; the $G$ proteins can take different routes to influence cellular processes. Once the $\mathrm{G} \alpha$ is released from the receptor, signaling can be prolonged by binding of a new $\mathrm{G}$ protein complex or receptor de-activation is initiated.

The G $\beta \gamma$ proteins are able to recruit G protein-coupled receptor kinases (GRK's) to the receptor [42]. Without a $G$ protein complex, the ligand-bound receptor is exposed for phosphorylation. The GRK's phosphorylate the receptor at Serine $(S)$ or Threonine (T) amino acids in the third intracellular loop or the C-terminal tail (steps three and four in Figure 1). The Ga protein-GTP is hydrolyzed back to GDP with the aid of so-called regulator proteins (RGS or regulators of G protein signaling). After this conversion, the $\beta \gamma$-parts can bind again. This regenerated inactive $G$ protein complex then resides on the membrane ready for a new interaction (step three and four in Figure 1).

Phosphorylation of the receptor stimulates $\beta$-arrestin to interact with the receptor. Betaarrestin signals the recruitment of clathrin and AP- 2 which then internalizes the receptor. What happens then depends on receptor type. For example, class A receptors lose $\beta$-arrestin, are dephosphorylated and recycled back to the membrane, whereas class $B$ receptors keep $\beta$-arrestin and are labeled (ubiquitylated) for degradation by the lysosome. Receptors that are recycled back to the plasma membrane are in open conformation, meaning that they are without a ligand and thus are ready to be re-activated and start the signal transduction again. [41, 43-45]

\section{Orthosteric and allosteric regulation of GPCRs}

The activation of the GPCR sets a whole chain of events in motion which ultimately leads to a cellular response. The target molecule, or agonist, that interacts with the receptor stabilizes the active conformation of the GPCR. However, with such a large diversity of molecules available, receptors have evolved to fit various molecules. For example, the class C GPCRs are known for their large extracellular domain, called the Venus Flytrap domain (VFT). This additional domain serves as an antenna, or trap, for many different molecules. The human sweet receptor which is a heterodimer of two class C GPCRs (hTAS1R2 and R3), has extracellular sites tuned for a wide range of molecules, including monosaccharides, disaccharides, polysaccharides, artificial and natural sweeteners and even sweet proteins $[25,46]$. These agonists all bind on different sites, either on the VFT, the hinge between the receptor and the VFT, or on the extracellular loops of the receptor. Every site on the receptor that is able to bind an agonist and stabilizes the GPCR into an active conformation 
Table 2, ligand types and effect on GPCR

\begin{tabular}{|c|c|c|c|}
\hline Ligand type & Binding site & Signaling effect & Example \\
\hline Full agonist & orthosteric & Maximal activation of GPCR & $\begin{array}{l}\text { Substance } P \text { is a agonist to the } \\
\text { Neurokinin receptor [47] }\end{array}$ \\
\hline Partial agonist & orthosteric & $\begin{array}{l}\text { Partial activation and partial } \\
\text { inactivation }\end{array}$ & $\begin{array}{l}\text { Cannabidiol is a partial agonist to the } \\
\text { dopamine receptor [48] }\end{array}$ \\
\hline Inverse agonist & orthosteric & Opposite effect of agonist & $\begin{array}{l}\text { Cannabidiol is a partial agonist to the } \\
\text { GPR12 receptor [49] }\end{array}$ \\
\hline $\begin{array}{l}\text { Competitive } \\
\text { antagonist }\end{array}$ & orthosteric & $\begin{array}{l}\text { Competition between } \\
\text { agonist and antagonist for } \\
\text { the same site }\end{array}$ & $\begin{array}{l}\text { CP-96,345 is a non-peptide antagonist } \\
\text { to NK1 [50] }\end{array}$ \\
\hline $\begin{array}{l}\text { Irreversible } \\
\text { antagonist }\end{array}$ & orthosteric & Termination of signaling & $\begin{array}{l}\text { Phenoxybenzamine is an irreversible } \\
\text { antagonist to } \alpha \text {-adrenoreceptor [51] }\end{array}$ \\
\hline PAM & allosteric & & IMP or GMP and the hTAS1R1 $[27,28]$ \\
\hline NAM & allosteric & & Lactisole and the hTAS1R3 [27] \\
\hline
\end{tabular}

is called an orthosteric site. Orthosteric sites, therefore, have direct effect on the structure and activation of the GPCR. Besides agonists, other molecules may interact with the orthosteric sites. Some molecules partially stabilize the GPCR structure and are, therefore, called partial agonists resulting in a partial or lowered activation compared to the full agonists. Molecules that bind to the orthosteric sites, but have no stabilizing effect on the GPCR structure are called antagonists. They are like keys that cannot turn in the lock and therefore do not set the GPCR chain of events into action.

Besides orthosteric sites, the GPCR may also have allosteric sites. These are distinct from the orthosteric sites, but have an effect on receptor stabilization. The target molecules for the allosteric sites are called allosteric modulators. When an allosteric modulator binds to the allosteric site, it changes the affinity and activity of the orthosteric site. There are positive allosteric modulators (PAM's) which aid an agonist to stabilize the GPCR active conformation of the receptor, and there are negative allosteric modulators (NAM's) which obstruct the stabilization of the GPCR by for example locking the 7TM domains in an inactive conformation.

\section{G protein signalling pathways}

Depending on the type and functionality of the receptors, their signaling is steered by the $G$ proteins that are co-expressed in a specific tissue type. In $\mathrm{G}$ protein signaling there are two independent pathways; the $G \alpha$ and the $G \beta \gamma$ pathway. Both the $G \alpha$ and the $G \beta \gamma$ pathways will now be discussed with a focus on olfaction and taste receptors. 


\section{Ga pathways}

There are many different $\mathrm{G} \alpha$ proteins known in humans. These $\mathrm{G} \alpha$ proteins may be coexpressed in the same tissue or cells and may even couple to the same receptor. This variability of the $\mathrm{G}$ protein coupling is called biased agonism (where the agonist-induced conformational changes set a preference for a Ga-type)[52, 53]. For example, the $G \alpha_{\text {Gustducin }}$ is expressed in gustatory tissue in combination with taste receptors [54]. But taste receptors are able to couple other $\mathrm{G} \alpha$-proteins as well, like $\mathrm{G} \alpha_{\mathrm{i} / \mathrm{o}}$ [55] and $\mathrm{G \alpha}_{12}$ [56]. Different agonists can, therefore, bind to the same taste receptor but induce different cellular effects.

Table 3, Human G protein families

\begin{tabular}{|c|c|c|c|c|c|}
\hline $\begin{array}{l}\text { Galpha } \\
\text { family }\end{array}$ & members & $\begin{array}{l}\text { Signal } \\
\text { transduction }\end{array}$ & $\begin{array}{l}\text { Second } \\
\text { messengers }\end{array}$ & Example type of receptors & Refs \\
\hline Gs & $G \alpha_{s^{\prime}} G \alpha_{\text {oLF }}$ & $\begin{array}{l}\text { Activation of } \\
\text { adenylate cyclase }\end{array}$ & cAMP up & Olfactory receptors & {$[57]$} \\
\hline \multirow[t]{2}{*}{$\mathrm{Gi}$} & $\begin{array}{l}G \alpha_{i}, G \alpha_{o^{\prime}} \\
G \alpha_{z}\end{array}$ & $\begin{array}{l}\text { Inhibition of } \\
\text { adenylate cyclase }\end{array}$ & cAMP down & $\begin{array}{l}\text { Chemokine receptors, } \\
\text { Adrenoreceptors, Serotonin } \\
5-\mathrm{HT}_{1} \text { receptors, Histamine } \\
\mathrm{H}_{3} \text { and } \mathrm{H}_{4} \text {, Dopamine } \mathrm{D}_{2} \text {-like } \\
\text { receptors }\end{array}$ & [58-63] \\
\hline & $\begin{array}{l}\mathrm{G} \alpha_{\text {Transducin' }} \\
\mathrm{G} \alpha_{\text {Gustducin }}\end{array}$ & $\begin{array}{l}\text { Activation of } \\
\text { phosphodiesterase }\end{array}$ & $\begin{array}{l}\text { cAMP/cGMP } \\
\text { down }\end{array}$ & Taste receptors and rhodopsins & {$[54,64-66]$} \\
\hline $\mathrm{Gq}$ & $\begin{array}{l}\mathrm{G} \alpha_{q^{\prime}}, \mathrm{G} \alpha_{11^{\prime}} \\
\mathrm{G} \alpha_{14^{\prime}} \\
\mathrm{G} \alpha_{15^{\prime}}, \mathrm{G} \alpha_{16}\end{array}$ & $\begin{array}{l}\text { Activation of } \\
\text { phospholipase } \mathrm{C}, \\
\mathrm{IP}_{3} \text { pathway }\end{array}$ & $\begin{array}{l}\mathrm{IP}_{3} \text { and } \\
\text { calcium up }\end{array}$ & $\begin{array}{l}\mathrm{NK} 1 \text {, Beta-adrenoreceptors; } \\
\text { Serotonin } 5-\mathrm{HT}_{4}, 5-\mathrm{HT}_{6} \text { and } \\
5-\mathrm{HT}_{7} \text {; Dopamine } \mathrm{D}_{1} \text {-like } \\
\text { receptors, Histamine } \mathrm{H}_{2}\end{array}$ & {$[67,68]$} \\
\hline $\mathrm{G} 12 / 13$ & $\mathrm{G} \alpha_{12}, \mathrm{G} \alpha_{13}$ & $\begin{array}{l}\text { Activation of the } \\
\text { Rho family of } \\
\text { GTPases }\end{array}$ & & $\begin{array}{l}\text { NK1 and various proteins } \\
\text { responsible for cytoskeleton } \\
\text { regulation }\end{array}$ & {$[67,69]$} \\
\hline \multicolumn{6}{|c|}{ Gbeta and gamma family } \\
\hline $\mathrm{G} \beta_{3} / \mathrm{G} \gamma_{13}$ & & $\begin{array}{l}\text { Activation of } \\
\text { phospholipase } C, \\
\mathrm{IP}_{3} \text { pathway }\end{array}$ & calcium up & Taste receptors & {$[66]$} \\
\hline
\end{tabular}

G $\alpha$ proteins can be divided into four classes as shown in Table 3. Each type of Ga leads to a different signaling pathway and these will be discussed separately.

The $\mathrm{G} \alpha_{s}$-type proteins activate the cAMP pathway, named after the second messenger cyclic adenosine mono-phosphate, and target the membrane-bound adenyl cyclase (AC). The AC catalyzes the conversion of ATP to CAMP. When CAMP increases inside the cell it can exert its effect via protein kinase $A$ (PKA) or it can bind to cyclic-nucleotide-gated (CNG) ion channels in the cell membrane. When these ion channels are activated they transport $\mathrm{Na}^{+}$or $\mathrm{Ca}^{2+}$ ions across the plasma membrane. The influx of these ions depolarize the cell and activate $\mathrm{Ca}^{2+}$ and $\mathrm{Cl}^{-}$gated ion channels. A sequence of polarization and de-polarization events is initiated, allowing a signal to travel via neurons and synapses to the brain. These 
action potentials are found for example in olfactory sensory neurons. When an odorant binds to an olfactory receptor (OR), the $\mathrm{G \alpha}_{\mathrm{OLF}}$ pathway is activated leading to an increase in cAMP production $[57,70]$.

In contrast, $G \alpha_{i / o}$ proteins inhibit AC activity and thereby decrease levels of cAMP. The activity of PKA is decreased causing opposite effects compared to $G \alpha_{s}$ type signalling. This group of $\mathrm{G} \alpha$ proteins also includes $\mathrm{G} \alpha_{\text {Transducin }}$ and $\mathrm{G} \alpha_{\text {Gustducin }}$. Both $\mathrm{G}$ proteins are very similar in structure and function [65] with transducin being involved in photo transduction and eyesight and gustducin in taste perception. Activation of $\mathrm{Ga}_{\text {Transducin/Gustducin }}$ leads to the phosphodiesterase-mediated decrease in cAMP (see Figure 2).

The $G \alpha_{q^{\prime}} G \alpha_{11}$ but also $G \alpha_{15 / 16}$ proteins that activate the inositol triphosphate or IP ${ }_{3}$ pathway target membrane bound phospholipase C (PLC). The PLC hydrolyses phosphatidylinositol 4,5-bisphosphate $\left(\mathrm{PIP}_{2}\right.$ ) to $\mathrm{IP}_{3}$ and diacylglycerol (DAG). DAG targets protein kinase $\mathrm{C}$ (PKC) which plays a role in phosphorylation of other effectors leading to a cellular response [71]. $I_{3}$ can activate the $I P_{3}$ receptors/ion channels on the endoplasmic reticulum (ER) within the cell which then opens to release calcium ions into the cytoplasm.

\section{Chimeric Ga pathway}

In specialized tissues like the sensory epithelium of the nose and tongue, where olfactory and taste receptors are expressed, highly-specific Ga type subunits bind to these receptors. The downstream signaling pathways of $G \alpha_{\text {Gustducin }}$ and $G \alpha_{\text {oLF }}$ involve many tissue-specific genes that are not readily expressed in the cell lines commonly used in high throughput screening assays $[72,73]$. In order to steer the signalling pathway of these receptor types towards a more suitable route for GPCR screening cell lines, G $\alpha$ chimera's have been developed. Examples are $\mathrm{G \alpha}_{15}$ (human) and $\mathrm{G \alpha}_{16}$ (rodent), which can promiscuously bind to many receptors [74] and have been used as templates for these chimera's. A C-terminal chimeric $\mathrm{G} \alpha$ protein of $\mathrm{G} \alpha_{16}$ with 44 residues of gustducin $\left(\mathrm{G \alpha}_{16 \mathrm{GUST} 44}\right)$ is able to functionally bind with heterologous expressed bitter receptors [75], see Figure 2B. Likewise, a chimera of $\mathrm{G \alpha}_{15}$ with 47 residues of $\mathrm{G} \alpha_{\text {oLf }}\left(\mathrm{G \alpha}_{15 \mathrm{OLF} 47}\right.$, [76]) has been developed for the coupling to odorant receptors and steer the pathway towards calcium.

\section{GBy pathway}

Besides the $\mathrm{G} \alpha$ signal transduction pathways, the $\mathrm{G} \beta \gamma$ proteins also play a part in the cellular response. G $\beta \gamma$ may affect ion channels, PLC and other signaling effectors. In bitter taste receptors the $G \beta_{3}$ and $G \gamma_{13}$ proteins activate Phospholipase C Beta 2 (PLC 32 ) to activate the $\mathrm{IP}_{3}$ signaling route and increase intracellular calcium. 


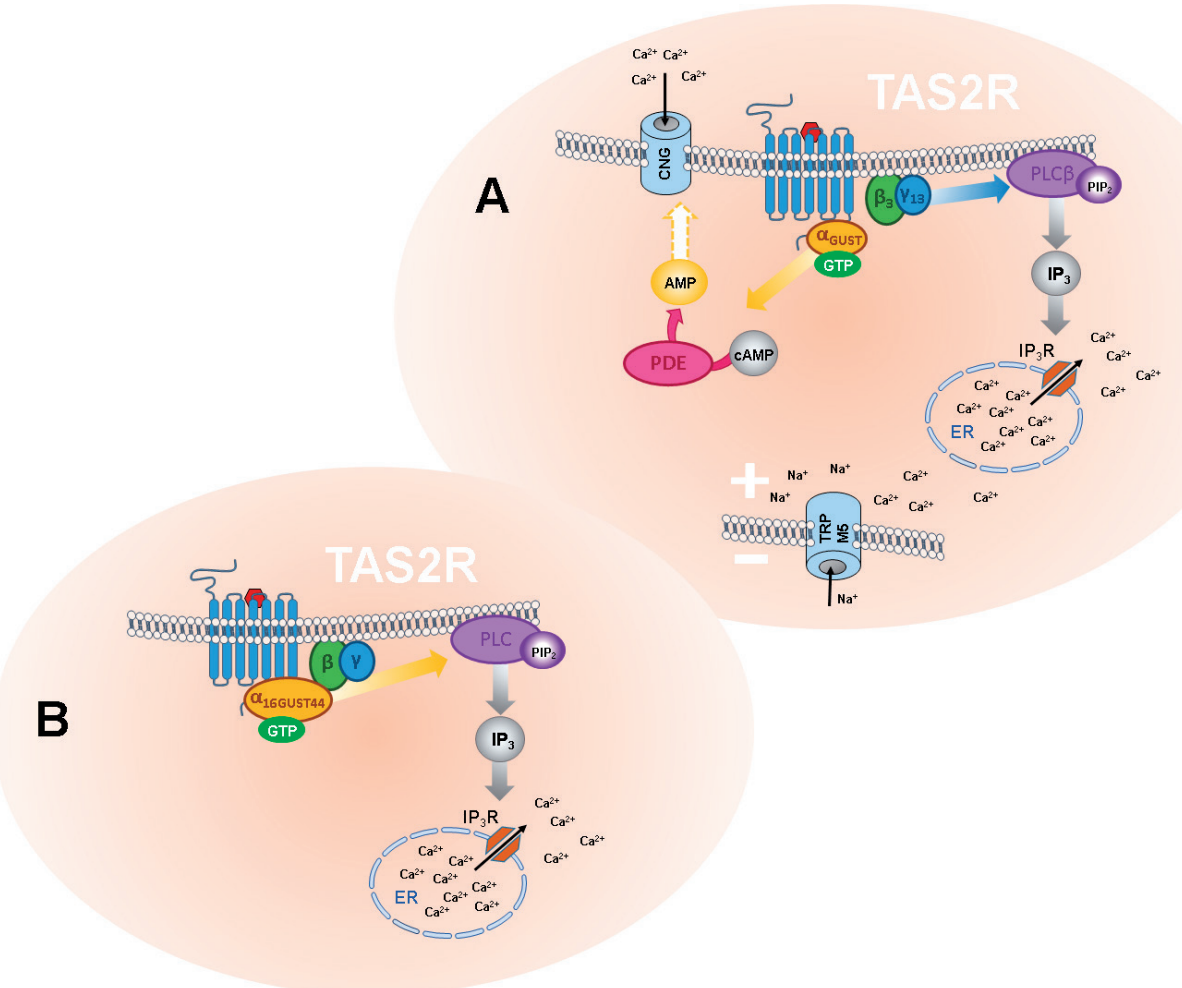

Figure 2, A Signalling pathway in bitter taste cells. $G \alpha_{\text {Gustducin }}$ activates phosphodiesterase (PDE) which converts CAMP into AMP. The cyclic nucleotide inhibited ion channels (CNG) which are blocked by cAMP can open and allow passage of calcium ion into the cells. At the same time, the $G \beta \gamma_{\text {Gustducin }}$ proteins activate phospholipase beta 2 to initiate the $\mathrm{IP}_{3}$ pathway increasing calcium from intracellular calcium stores. The increase of intracellular calcium from both $\mathrm{G} \alpha$ and $\mathrm{G} \beta \gamma$ pathways stimulate TRPM5 ion channels to depolarize the cell initiating an action potential.[66] [77] B, Signaling pathway in screening assay cells. Using chimera $G$ protein $\mathrm{G}_{16 \mathrm{GUST4}}$ the bitter taste receptor can directly couple to the $\mathrm{IP}_{3}$ pathway resulting in a calcium increase in HEK293 cells [75]

\section{Reverse transfection}

Receptor research in general and receptor-ligand pairing research in particular require an effective and efficient screening approach. Reverse transfection is one such approach with huge potential. Almost two decades ago, Ziauddin and Sabatini published a breakthrough technology which had the potential to greatly improve our efficiency in receptor analysis research; a simple method to make a living cell array on a carrier surface [78]. Later, this method was adapted to create various modified versions of reverse transfected cell arrays [79], including the printing of RNAi [80], cDNA, viral vectors and even compound libraries. All sorts of reverse transfected cell arrays have since been designed over the years, printed on for example, the surface of a glass microscope slide or inside a well of a 96 wells plate 
[81]. Methods have been developed to prepare highly densely populated transfected cell microarrays $[80,82]$ by creating hydrophobic barriers between the printed DNA spots. These barriers prevent cross-contamination of arrayed spots and the migration of cells. Some groups have even prepared an integrated microfluidic cell-culture platform that allows for on-chip transfections in small reaction chambers $[83,84]$. The level of complication and sophistication that has been reached over the years is extensive and has resulted in specialized niches. One of these niches includes the expression of GPCRs in a cell array format which was previously done by printing DNA arrays inside multi-well plates [81] or in fabricated micro reaction wells [85]. The combination of a GPCR cell array in a microfluidic flowcell is, however, a new field of application which will be explored in this thesis.

\section{GPCR screening assays}

GPCRs are important pharmacological targets (30-40\% of medicines target a GPCR, [8688]) and are therefore commonly used in high throughput screening assays to develop new drugs. These assays test compound libraries, either natural or synthetic, in an automated manner [89]. Strategies to detect GPCR activation involve live cell assays expressing target GPCRs and monitoring of the GPCR activation chain of events. Events like changes in second messenger (calcium, cAMP or $\mathrm{IP}_{3}$ ) levels can be monitored [90, 91], using for example, beta-arrestin [92] or expressed reporter genes (CRE - luciferase, [93]). High throughput platforms are now available for almost every signalling pathway [94]. These micro-well plate based high-throughput screening platforms of compound libraries are well established. Furthermore, microarray and microfluidic technologies may hold potential for miniaturization, automation, and biosensor integration [94-96] (Figure 3).

\section{Receptomics}

The term receptomics was first coined in 2004 to describe the systematic study of the receptorome, i.e. the collection of receptors and ligand-gated ion channels that serve as sensors and potential drug targets[101, 102]. Covering an estimated $8 \%$ of the total human genome [101] this protein class is significantly represented. Receptor cell microarrays, especially in combination with microfluidics, are considered as a very promising road towards reducing the cost and increasing the throughput of screening receptor libraries [94]. Only few studies have combined GPCR microarrays with microfluidics so far. In some of those studies, each spot of the cell array was enclosed by a chamber that allowed individual fluidic control $[84,103,104]$. These micro-chambers mimic a multi-well plate where each well or chamber is individually addressable for measurements by different compounds/ samples. However, a set-up applying controlled fluidics to individual chambers is relatively complex and inefficient for a generic "receptomics-type" screening of GPCR libraries which aims to screen the entire library with the same compound or extract rather than a single receptor with a library of compounds [100, 105, 106]. 


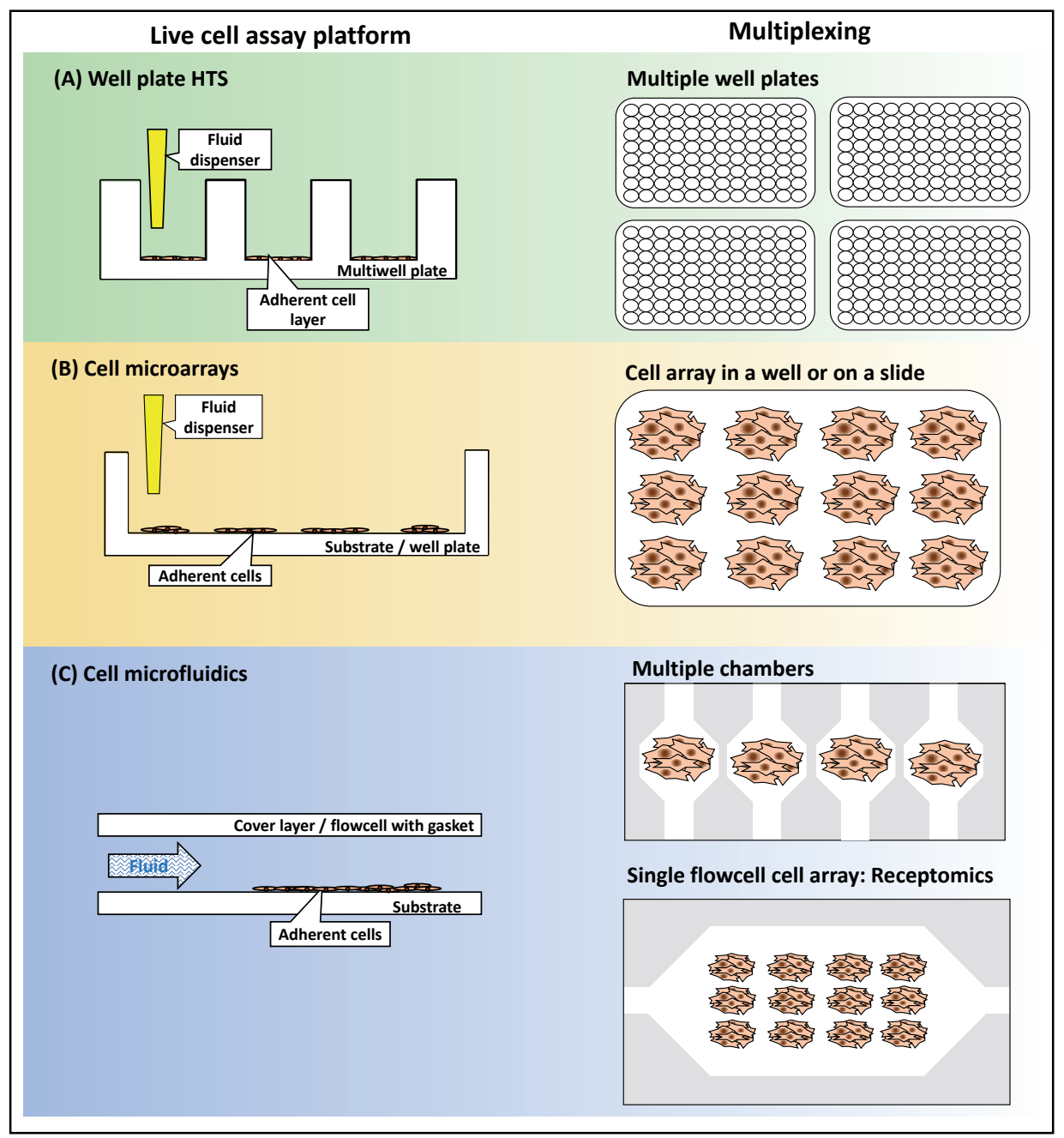

Figure 3, Receptor cell assay platforms (diagram adapted from Martins et al 2012). A, The multiwell plate format; cells are seeded on the surface of a multi well plate and (top-down) transfected with receptor coding plasmid DNA. Calcium dye loading, subsequent washing steps and test sample dosing are done using fluid dispensers. The test conditions are multiplexed by using multiple wells and multiple plates making this platform compatible with automation but at relatively high costs and low assay flexibility [90]. B, Receptor cell arrays are created within one well chamber by printing cells [97] or by printing receptor coding plasmid DNA into a micro well and by reverse transfection with cells $[81,85]$. Arrays are co-transfected with fluorescent indicator proteins to locate the array. Array washing and sample dosing are done using fluid dispensers. This combines the HTS nature of multi-well plates with the possibility to screen multiple receptors and controls in a single well. C, In microfluidic platforms the cells are cultured or assembled into a microfluidic system [98, 99]. A system can be designed so that different channels address different chambers with (reverse transfected) cells analogous to $\mathbf{A}$ but with the option of repeated exposures [84] or a reverse transfected array can be enclosed in one large flowcell, sequentially addressing all spots at once with different samples analogous to $\mathbf{B}$ but with the option of repeated challenges and larger arrays [100]. 


\section{Large potential}

The function(s) and ligand-specificity of many GPCRs are still unknown. These receptors are therefore called "orphan" receptors $[1,107]$. Additionally, the large number of single nucleotide polymorphisms (SNP's) and splicing variants of the receptor or ion-channel genes further increases their diversity for potential ligands, as well as the potential to couple to different $G$ protein complexes [108-110]. As a consequence, the number of potential receptor conformations is remarkably high. Receptomics projects may promote the systematic screening of large compound libraries. Such treasure-hunt like projects have therefore the potential to deliver many new compound leads.

\section{Aims and layout of the thesis}

The receptomics platform described in this thesis has led to a novel enabling technology. The technology was developed to enable applications for sensing taste-relevant molecules and bioactivity of molecules in extracts by monitoring the activation of GPCRs. The combination of existing technologies, such as reverse transfection to create living cell arrays, calcium imaging, microfluidics to allow controlled and repeated sample exposure, and the development of specific software for the statistical analysis, could enable new types of GPCR studies that provides data in a novel format and allows novel applications. In this thesis the aim is to present the technical as well as biological development of a fully functional receptomics platform. We have studied exposure-controlled receptor responses in stable cell lines and in reverse transfected cell arrays; we have determined key parameters in gene dosing that control the sensitivity and robustness of the receptor responses; we have developed specialized software and statistical approaches for the analysis of injection series and we have laid out a methodology for the analysis of complex mixtures. This thesis also further discusses the potential directions of future developments, focussing in particular on the potential benefits of a microfluidic receptomics assay.

The chapters in this thesis cover different steps in the development of the receptomics technology.

In Chapter 2 we describe the general principles, setup and characterization of the microfluidic biosensor to measure GPCR activation via imaging of $\left[\mathrm{Ca}^{2+}\right]$ changes in recombinant human HEK293 cells. These cells expressed a combination of the Neurokinin 1-receptor and Cameleon YC3.6 protein as calcium indicator.

In Chapter $\mathbf{3}$ we show how the biosensor, coding for the transient receptor potential channel vanilloid 1 (TRPV1) ion channel and the Cameleon YC3.6 calcium sensor, can be used to determine bioactivity in chilli pepper varieties by measuring LC-MS-purified fractions in sequential injections as well as measuring bioactivity in the crude extract. 
In Chapter 4 we provide an extensive characterization of the preparation and measurement of reverse transfected cell arrays using fluorescent proteins. The response of the Neurokinin 1-receptor in relation to its gene dose in reverse transfection is studied as well as the response behaviour during repeated activations.

In Chapter $\mathbf{5}$ we switch to the bitter taste receptor family and aim to enhance the sensitivity and robustness of the receptor assay by changing the calcium sensor type and adjusting the sensor gene dose.

In Chapter 6 we present a linear mixed model for a spot-based analysis and comparison of receptor responses. A host cell response, induced by ATP, is used to show that specific bitter receptor responses from compound spikes are additive to the host cell response and can be retrieved from a host cell response signal by means of comparative analysis.

The general discussion places the receptomics technology in the context of existing receptor screening technologies and discusses the current limitations and potential benefits. The microfluidic nature of the platform is discussed in relation to potential novel applications and the promise of new technological developments to overcome certain limitations is outlined. 

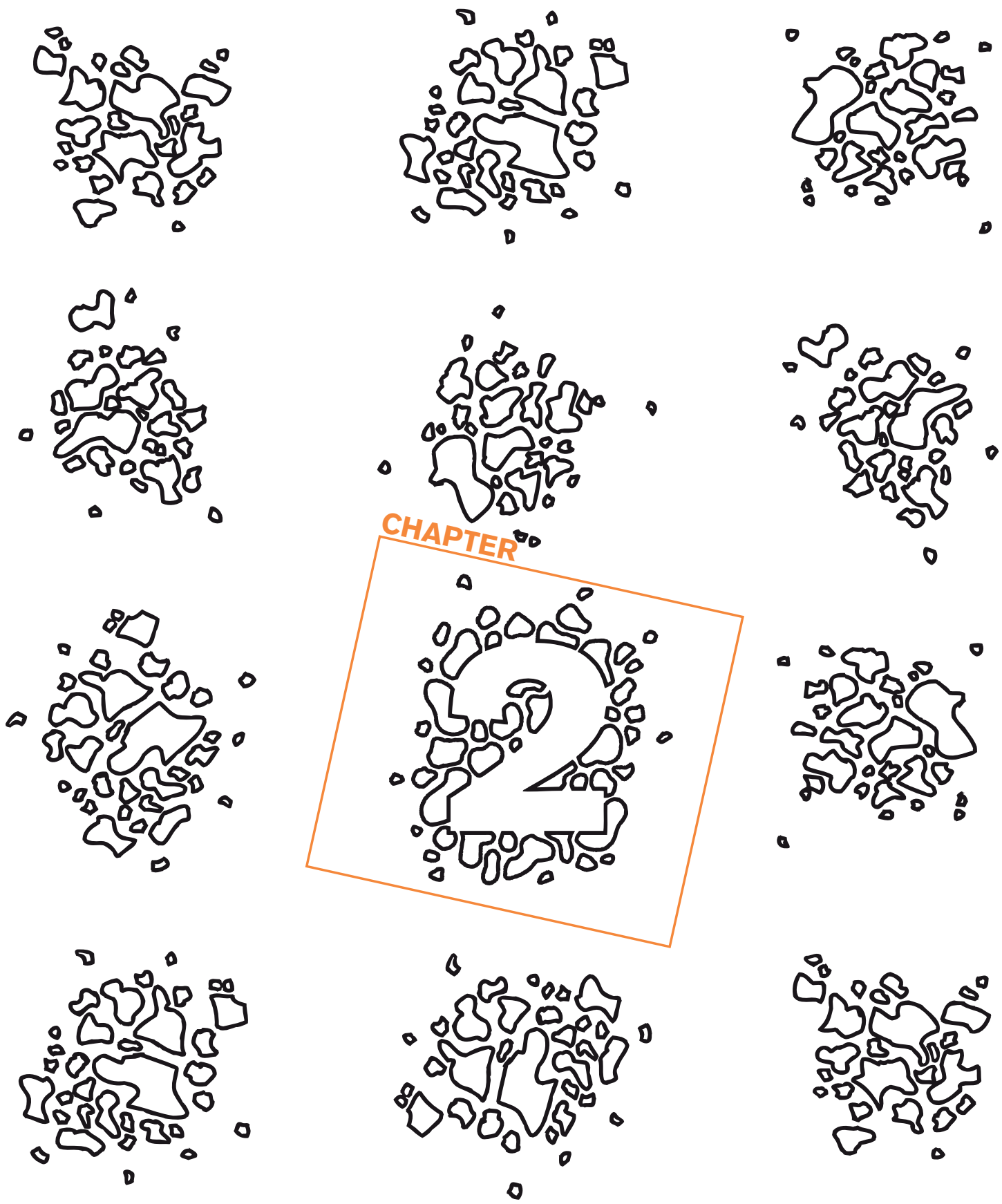

-
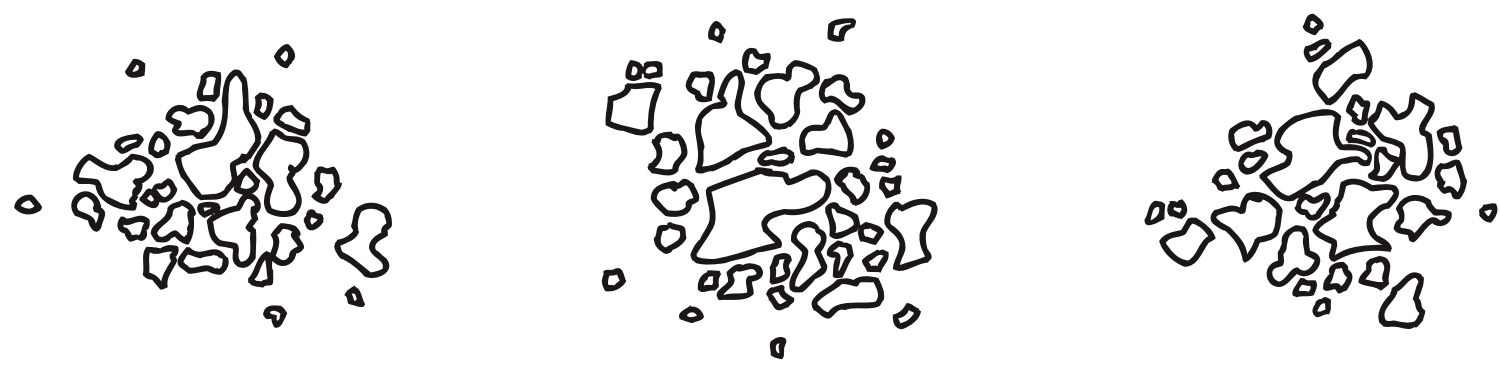


\section{A generic microfluidic biosensor of GPCR-activation - monitoring cytoplasmic $\mathrm{Ca}^{2+}$ flux in human HEK293 cells}

Margriet Roelse, Norbert C.A. de Ruijter, Elwin X. Vrouwe and Maarten A. Jongsma 


\section{Abstract}

Cell lines expressing recombinant G-protein coupled receptors (GPCRs) are activated by specific ligands resulting in transient $\left[\mathrm{Ca}^{2+}\right]$ rises that return to basal levels in 30-60 seconds. Yellow Cameleon 3.6 (YC3.6) is a genetically encoded calcium indicator which can be coexpressed to monitor these cytosolic [ $\left.\mathrm{Ca}^{2+}\right]$ changes in real-time using Förster (Fluorescence) resonance energy transfer (FRET). On this basis, we designed the prototype of a generic microfluidic biosensor of GPCR activation, imaging $\left[\mathrm{Ca}^{2+}\right]$ changes in recombinant human HEK293 cells, which express a combination of a GPCR (Neurokinin 1) and YC3.6. An internal reference for non-specifically induced $\left[\mathrm{Ca}^{2+}\right]$ changes were YC3.6 cells without GPCR but expressing a red fluorescent protein (mCherry) for identification. These cell lines were grown as a mixed population in a flow cell with a volume of $\sim 50 \mu$ and a flow cell surface of $170 \mathrm{~mm}^{2}$. Cells were activated by brief exposures to specific and non-specific analytes using an injection valve with a flexible sample volume (tested range 5 to $100 \mu$ ) at a flow speed of $100 \mu \mathrm{l} / \mathrm{min}$. A flow cell surface of $0.2 \mathrm{~mm}^{2}$ with 50 cells was imaged every 2-4 seconds to obtain signal kinetics. The lower limit of detection was $30 \mathrm{pM}$ Substance $P(S P, 2 \mathrm{pg} / 50 \mu \mathrm{l})$, and reproducible responses to repeated injections every 3 minutes were obtained at $1 \mathrm{nM}$ SP. This biosensor was designed for $\sim 50$ cells for statistical reasons, but at a lower limit of 1 receptor- and 1 reference-cell, specific ligand detection is still feasible. 


\section{Introduction}

G protein-coupled receptors (GPCRs) in humans represent a family of around 800 genes which play key roles in many physiological or disease-related processes and also determine our sense of smell and taste. For this reason these receptors are favorite targets for both the pharmaceutical and food industries [111-115]. Microtiter plate based high-throughput screening platforms are well established, but microfluidic technologies hold potential for miniaturization and automation that are predicted to redefine the format and application areas of GPCR screening assays [116-120].

The most common methods of measuring GPCR activation in vivo in real time are by recording concentration dynamics in generic secondary messengers such as $\left[\mathrm{Ca}^{2+}\right]$, cAMP and $\mathrm{IP}_{3}$ using various indicator dyes $[121,122]$. The concentration of cytosolic $\left[\mathrm{Ca}^{2+}\right]$ is very low (nM range) when cells are not triggered. External signals can cause the rapid release of micromolar concentrations of calcium into the cytosol from organelles like the endoplasmic reticulum (ER), mitochondria and nuclei or from extracellular sources [123]. Once released into the cytosol, the $\left[\mathrm{Ca}^{2+}\right]$ rise is transient and levels fall back to basal levels within less than one minute after the ligand is washed away due to uptake by the ER and mitochondria. In cell populations concentrations of cytosolic $\left[\mathrm{Ca}^{2+}\right]$ are directly related to the concentrations of the activating ligand, except for a subclass of GPCR receptors, which couple to Ga proteins (e.g. $G_{i / o^{\prime}}, G_{s^{\prime}} G_{12 / 13}$ ) that, depending on the cell line, actually inhibit this response or bypass it. In those cases, overexpression of specific promiscuous $\mathrm{G} \alpha$ proteins or CAMP-gated ion channels can steer the response towards the desired [ $\mathrm{Ca}^{2+}$ assay [124]. The duration of the $\left[\mathrm{Ca}^{2+}\right]$ transient depends a.o. on receptor sensitivity and ligand concentration and duration.

The increase of $\left[\mathrm{Ca}^{2+}\right]$ concentration in the cytosol resulting from GPCR signaling can be measured by small molecule organic indicators like fura-red, indo-1 and many others [122], but genetically encoded calcium indicators (GECls), such as cameleon and GECO can also be used [125-129]. The choice of the calcium indicator type largely depends on the spatial and temporal resolution that is required to monitor calcium signaling processes. Current advantages of small molecule indicators are their expanded dynamic range for measuring $\left[\mathrm{Ca}^{2+}\right]$ concentrations and faster response kinetics. The use of $\mathrm{GECls}$, however, offers advantages in other areas. Here, we used a $\mathrm{GECl}$, because it can be fused to any receptor protein of interest allowing the measurement of $\left[\mathrm{Ca}^{2+}\right]$ in micro-domains in its immediate vicinity. Furthermore, co-expression of $\mathrm{GECl}$ with receptor genes allows that only transfected cells are monitored. In case of stably transfected lines, the GECls are immediately available for measurements and do not leak out over time, which is an important advantage when they are used in diagnostics and microfluidic biosensors. Cameleon YC3.6 is a GECI with a suitable dynamic range for measuring calcium dynamics from GPCR activation, while displaying acceptable response kinetics $[130,131]$. Calcium signaling from YC3.6 is based 
on fluorescence energy transfer (FRET) that can occur when the two fluorescent proteins of the yellow cameleon molecule; CFP and YFP are in close vicinity. These fluorescent proteins are linked together by a calmodulin domain and M13 peptide. The M13 peptide binds calcium-bound calmodulin, and thereby changes the conformation of the sensory part upon calcium binding.

Co-expression of GECl and GPCR proteins can be achieved from a polyprotein by employing a $2 \mathrm{~A}$ polypeptide sequence between the two proteins [132]. This has the advantage that the $\mathrm{GECl}$ expression level is correlated with the GPCR density on the membrane. The $2 \mathrm{~A}$ peptide of 20 amino acids is cloned to link the coding sequences of the two recombinant proteins. During translation of the $2 \mathrm{~A}$ part of the polyprotein the ribosomal machinery fails to create a peptide bond between two specific amino acids of the $2 \mathrm{~A}$ peptide, but continues translation of the remaining mRNA molecule. This results in the expression of both proteins at equimolar levels $[133,134]$.

Despite their predicted importance only very few studies have shown successful methods of studying $\left[\mathrm{Ca}^{2+}\right.$ changes in microfluidic formats [135] and with recombinant expressed GPCRs $[129,136]$. These reports have neither explored the limits of detection, nor the dynamics of the cell response under different conditions. Also at a practical level, the robustness of the set-ups for automated, repeated dosage of analytes was not explored [116]. In this study we developed a biosensor of GPCR activation, based on the co-expression of a GPCR and a GECI in human HEK293 cells. A new commercially available microfluidic set up is described, which allows sequential injections of small and defined volumes of ligands at short time intervals, while maintaining a constant flow of medium across the cells contained in a resealable flow cell. As model GPCR, we used the Neurokinin 1 receptor (NK1) for this study. Upon interaction with its substrate Substance P (SP), the NK1 receptor activates the $\mathrm{G} \alpha$ subunit and effects downstream signalling by $\mathbb{I P}_{3}$, resulting in an increase of intracellular calcium ions by release from the ER [47, 67, 137]. Calcium responses are monitored in real-time using a confocal fluorescent microscope setup. Reference cells monitoring endogenous activation of the cell line at the experimental conditions, thus allow determination of the specificity of activation in relation to the GPCR response. Addition of a known concentration of a fluorescent marker to the flow cell microfluidics provides a semiquantitative real-time correlation of extracellular ligand concentrations to intracellular $\left[\mathrm{Ca}^{2+}\right]$ concentration changes. 


\section{Material and Methods}

\section{DNA constructs}

The NK1 receptor gene was purchased at the UMR cDNA Resource Center of the University of Missouri-Rolla, USA (cat. Nr. TACR100000). The YC3.6 gene in pCDNA3 (Invitrogen) was obtained from Roger Tsien (USA). The NK1 gene was cloned into pSNAPf (New England Biolabs) without stop codon using the EcoRV and EcoRI sites of MCS1. Oligo's of T2A (GAATTCGAGGGCAGAGGAAGTCTTCTAACATGC GGTGACGTGGAGGAGAATCCCGGCCCTGGATCC=EGRGSLLTCGDVEENPGP) and T2ANC (GAATTCGAGGGCAGAGGAAGTCTTCTAACATGCGGTGACGTGGAGGAGAAT GCCGCACCTGGATCC=EGRGSLLTCGDVEENAAP) were hybridized and cloned into pSNAPf using ECoRI and BamHI replacing the SNAPf gene. The YC3.6 gene is cloned without start codon, but with stop codon into MCS2 using BamHI and Xhol. Control construct mCherryT2A-YC3.6 was cloned by PCR (FW 5'ATATGGCGCGCC ATGGTGAGCAAGGGCGAGGA and RV 5' ATATGAATTCCTTGTA CAGCTCGTCCATGC) from plasmid pENTRmCherry (Life Technologies Europe BV, Bleiswijk, NL), using Ascl and EcoRI restriction sites. Plasmids containing single CFP and YFP; pcDNA3-CFP (Addgene plasmid 13030) and pcDNA3-YFP (Addgene plasmid 13033) were obtained from Addgene Inc.

\section{Cell culture and transfection}

HEK293 cells (Invitrogen, 11631-017) were grown as a monolayer in cell culture medium (Dulbecco's Modified Eagle Medium (DMEM) containing 1x MEM non-essential Amino Acids, 1x Penicillin-Streptomycin (Invitrogen, 15140-122) and 10\% Fetal bovine serum FBS (Invitrogen, 26140)). Cells were transfected with expression vectors pNK1-T2A-YC3.6, pNK1T2ANC-YC3.6 and mCherry-T2A-YC3.6 using Effectene transfection reagent (Qiagen 301425). Selection with Geneticin at $600 \mu \mathrm{g} / \mathrm{ml}$ (Invitrogen) was started 24 hour after transfection and maintained for several weeks until stable expressing cell cultures were obtained. From a stable expressing cell culture a clonal line with intermediate YC3.6 expression, visually identified based on mean fluorescence intensity, was selected.

\section{Western blotting}

Cells were harvested at about $80 \%$ confluency, and resuspended in 1/5 volume RIPA buffer (Sigma-Aldrich, R0278) containing complete protease inhibitor cocktail (Roche 11697498 001). Samples were denatured in reducing SDS-PAGE sample buffer, separated on $12.5 \%$ SDS PAGE gel and transferred to nitrocellulose membrane by Western blotting. The GFP protein and spectral variants CFP and YFP were detected with mouse anti-GFP (Roche 11 814460 001) 1:5000 diluted in blocking buffer and sheep anti-mouse HRP (Jackson Immuno 515-035-062) 1:5000 diluted in blocking buffer. 


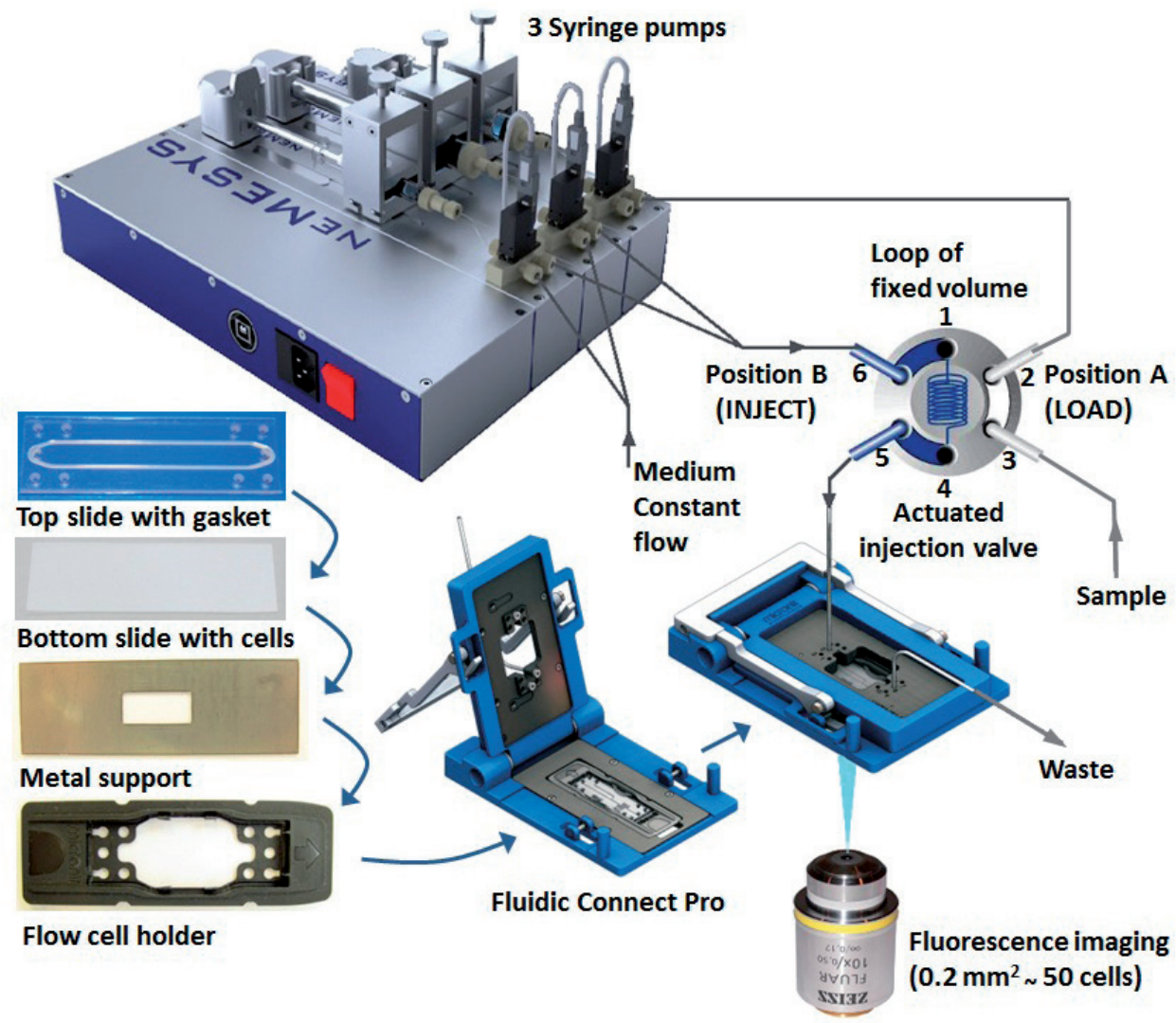

Figure 1, Diagram of the microfluidic biosensor setup. The microfluidic flow cell of $50 \mu \mathrm{l}$ is assembled in the blue Fluidic Connect Pro flow cell holder by mounting the indicated parts. Closing the holder connects the capillaries for dosing media and samples. Two syringe pumps maintain a constant flow of cell media by alternated uptake and dosing into the flow cell through ports 5 and 6 of the injection valve. Ports 2 and 3 are used to fill and empty/wash an injection loop using the third syringe. Switching the injection valve from LOAD to INJECT assures that samples of various volumes depending on the loop size are injected into the flow cell and vice versa that the loop can be reloaded. Fluorescence changes in the cell monolayer are imaged from below.

\section{Flow cell assembly and microfluidics}

All imaging was performed in resealable borosilicate glass flow cells (Micronit Microfluidics B.V., Enschede, The Netherlands). Temperatures were maintained at $22^{\circ} \mathrm{C}$ (RT) unless otherwise noted. Recombinant HEK293 cells were seeded on a disposable $15 \times 45 \mathrm{~mm}^{2}$ borosilicate cover-slide of standard (1.5 \#) $175 \mu \mathrm{m}$ thickness, coated with $100 \mu \mathrm{g} / \mathrm{ml}$ poly-LLysine (Sigma P6282) at a density of $\sim 3 \times 10^{5}$ cells $/ \mathrm{ml}$. The slides were incubated overnight in a sterile petridish in a $\mathrm{CO}_{2}$ incubator at $37^{\circ} \mathrm{C}$. Next, a re-usable borosilicate glass slide of $15 \mathrm{x}$ $45 \mathrm{~mm}^{2}$ and $1.1 \mathrm{~mm}$ thickness with a central $0.3 \mathrm{~mm}$ high gasket of $5 \times 43 \mathrm{~mm}$ (defining a flow cell inner surface area of $\sim 170 \mathrm{~mm}^{2}$ ) was placed over the cover-slide with cells to create the flow cell with cells inside (Fig. 1, Micronit Microfluidics BV, Enschede, The Netherlands). Two 
fluidic ports in the slide, $40 \mathrm{~mm}$ apart, served for microfluidic connections. The assembled flow cell was placed on a metal supporting plate of $15 \times 45 \mathrm{~mm}^{2}$ with opening of $5 \times 11 \mathrm{~mm}^{2}$ at the bottom to prevent deformation of the thin lower cover slide. The slides were sealed together in a Fluidic Connect Pro flow cell holder (Micronit Microfluidics B.V., Enschede, the Netherlands), which allowed immediate fluidic connection of the flow cell to a set of syringe pumps (Nemesis, Cetoni $\mathrm{GmbH}$ ) that controlled a constant flow of cell culture medium (100 $\mu \mathrm{l} / \mathrm{min}$; port 5 and 6 in Fig. 1). Injection of samples loaded in a sample loop was performed using an injection valve (Upchurch, V-451); 5-100 $\mu$ loop volume between port 1 and 4 for injection and port 2 and 3 for loading and rinsing of the sample loop.

\section{FRET imaging using confocal laser scanning microscopy}

Imaging of cameleon YC3.6 was performed with a Zeiss LSM 510-META 18 confocal laser scanning microscope on an Axiovert 200M equipped with a 20x 0.5 NA Plan-Neofluar DIC objective using the $458 \mathrm{~nm}$ line from a $30 \mathrm{~mW}$ Ar laser. With a series of detectors in the META channel a stack of $10.7 \mathrm{~nm}$ spectrally resolved images were recorded, called Lambda Stacks (465-610 nm). This allowed pixel-by-pixel collection and separation of the simultaneously recorded CFP and YFP emissions, based on reference spectra taken at identical imaging settings. Imaging was done at the cellular mid-plane with wide pinholes of 2.91 AU (Airy Unit) corresponding with a $10 \mu \mathrm{m}$ thick optical slice. The emission fingerprintbased CFP and YFP intensities of regions of interest were plotted in 2 second interval time series and their ratiometric FRET signals analysed. For this, HEK293 cells were transfected with reference plasmids pcDNA-CFP or pcDNA-YFP. Both cultures were used to record reference lambda stacks required for spectral unmixing at $458 \mathrm{~nm}$ excitation. An additional reference emission spectrum for fluorescein was recorded at $458 \mathrm{~nm}$ with imaging settings as used during experiments, to allow spectral unmixing in the presence of this internal dilution standard. Fluorescein concentrations were below saturating concentrations in all experiments to avoid errors in spectral unmixing. Confocal imaging of TAMRA-labelled Substance P (SPTAMRA, Anaspec cat nr. 61202) was done with HeNe laser of $543 \mathrm{~nm}$ and pinhole at $1 \mathrm{AU}$ to obtain the maximal z-resolution.

\section{Results and discussion}

\section{DNA constructs for biosensor cell lines}

To enable a $\mathrm{GECl}$-based system, genetic constructs were developed to co-express a model G-protein coupled receptor, Neurokinin 1 (NK1), and the GECl Cameleon YC3.6 from a single transcript, but as separate proteins (Fig. 2A). The two genes were separated by either the native T2A peptide linker, resulting in two translation products, or the mutated non-cleavable version T2ANC, resulting in a single multiprotein [132-134, 138]. The aim of using a $2 \mathrm{~A}$ construct was to obtain correlated expression levels of the receptor unit and the 

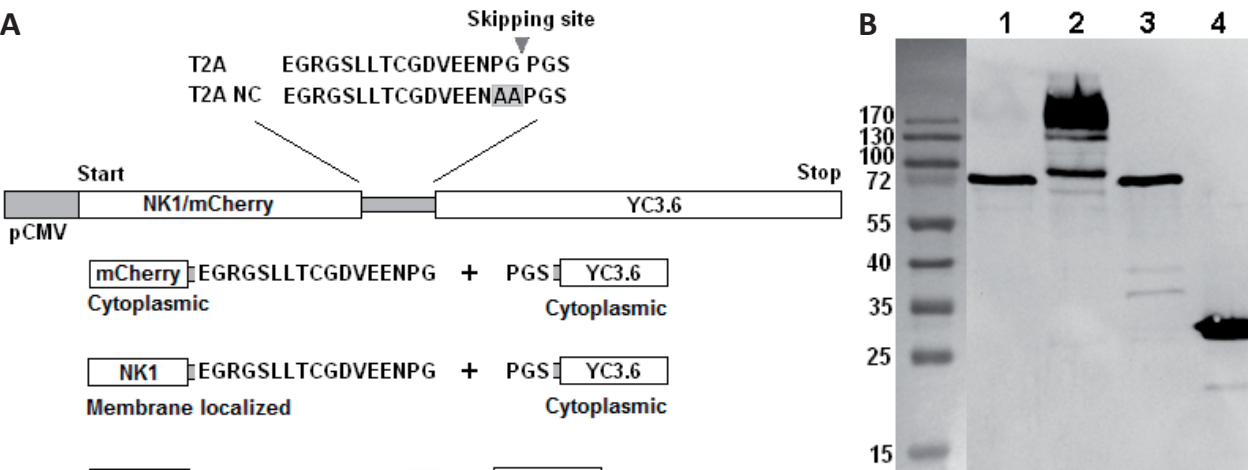

\begin{tabular}{|ll|}
\hline NK1 EGRGSLLTCGDENAAPGS & YC3.6 \\
\hline
\end{tabular}

Membrane localized

Figure 2, DNA and protein constructs to generate green GPCR and red reference cells. A, Top bar the DNA construct used for the transformation of HEK293 cells with either a GPCR target receptor (NK1) as biosensor or a red protein (mCherry) to tag reference cells. Lower three bars represent the expressed proteins. B, Western blot identifying YC3.6 containing polypeptides. Protein extracts of HEK293 cell lines expressing various constructs were separated by $12.5 \%$ SDS-PAGE, blotted to nitrocellulose membrane, and detected with anti-GFP antibody. The molecular weight of GFP is 27kDa, YC3.6 is $72 \mathrm{kDa}$ and NK1 is $45 \mathrm{kDa}$. Lane 1: pNK1-T2A-YC3.6; Lane 2: pNK1-T2ANC-YC3.6; Lane 3: YC3.6; Lane 4: GFP.

genetically encoded calcium indicator. The aim of using the T2ANC construct was to allow the visualization of the receptor on the plasma membrane and creating a single molecule capable of both extracellular sensing and monitoring of intracellular $\left[\mathrm{Ca}^{2+}\right]$ proximal to the plasma membrane.

The HEK293 cell line used to express these DNA constructs also carries native receptors on its surface which can also generate $\left[\mathrm{Ca}^{2+}\right]$ changes signals which may interfere with the signal from the NK1 receptor. To distinguish specific responses induced by the targeted receptor from non-specific cell responses, it is necessary, to measure in parallel reference cells, which do not ectopically express any recombinant $\left[\mathrm{Ca}^{2+}\right]$ changes inducing receptor, but do possess the fluorescent calcium reporter YC3.6. To recognize such cells we replaced the gene for the receptor with the one encoding red fluorescent protein, mCherry (Fig. 2A).

\section{Subcellular localization of the membrane receptor and calcium-indicator protein}

For all constructs, stable HEK293 clonal cell lines were made by selecting recombinant cell lines with stable integrated plasmid DNA and sub-culturing into a clonal line. The T2A and T2ANC peptides determine whether the protein fusions are expressed as one or two separate proteins. To verify the presence of the expected translation products and the efficiency of the T2A-induced ribosome skipping, protein extracts were analysed by Western blot using an antibody against GFP and spectral variants CFP and YFP (Fig. 2B). A YC3.6 expressing cell line (without the mCherry protein or receptor) showed a $72 \mathrm{kDa}$ band, 

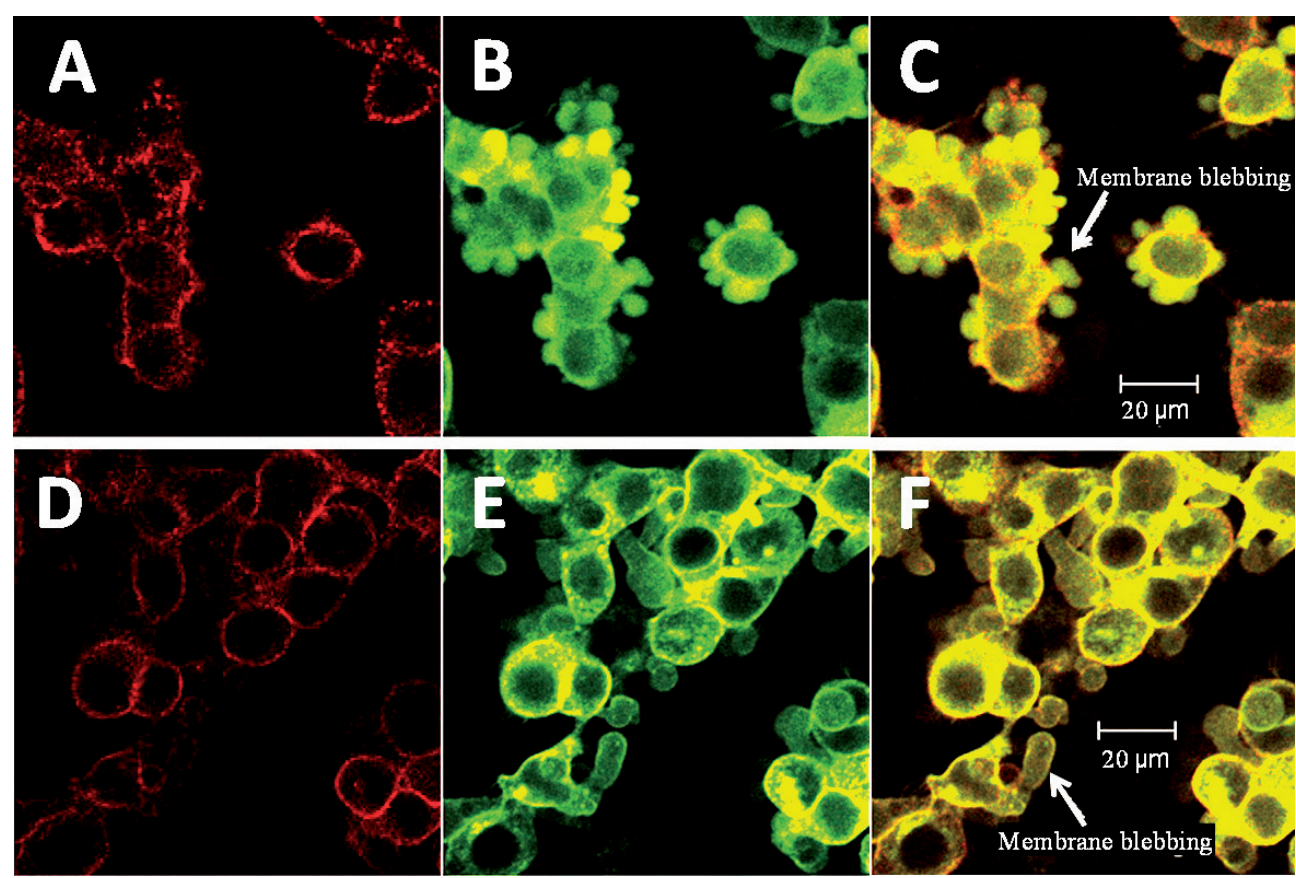

Figure 3, Confocal images of HEK 293 cells expressing cytoplasmic cameleon from NK1-T2A-YC3.6. A, B and C, and membrane associated cameleon from NK1-T2ANC-YC3.6. D, E and F, shortly after adding 50nM SPTAMRA. Pictures $\mathbf{A}$ and $\mathbf{D}$ show SPTAMRA localized on the cell membrane by excitation at $543 \mathrm{~nm}$. B and E show cameleon YC3.6 localization by direct excitation of YFP at $514 \mathrm{~nm}$. B shows cytoplasmic localization of YC3.6 and $\mathbf{E}$ shows localization of YC3.6 on the membrane and in intracellular compartments like the ER. $\mathbf{C}$ and $\mathbf{F}$ are overlay images of $\mathbf{A}+\mathbf{B}$ and $\mathbf{D}+\mathbf{E}$ respectively. SP-induced membrane blebbing is indicated.

which corresponds with the expected size of the cameleon protein. The cell line expressing the NK1 receptor and YC3.6 linked by the T2A polyprotein linker showed a single band with a size of $72 \mathrm{kDa}$ as well, which indicates that the first protein, the NK1 receptor, is fully cleaved from YC3.6 and that T2A-mediated ribosome skipping is very efficient. The cell line expressing the NK1 receptor and YC3.6 linked by the non-cleavable T2ANC peptide was expected to yield a fusion protein of $117 \mathrm{kDa}$. Next to two minor bands of $100 \mathrm{kDa}$ and $130 \mathrm{kDa}$ most signal, however, is of higher molecular weight. Since cameleon is fused to a membrane protein, which is known to resist complete SDS denaturation, it probably represents poorly dissolved fusion protein.

To demonstrate the functional plasma membrane localization of the NK1 receptor, SPTAMRA was used [139]. Confocal imaging showed that SPTAMRA is bound to the plasma membrane of cells expressing recombinant NK1 receptor from both T2A and T2ANC constructs (Fig. 3A, D). SPTAMRA did not bind to the membrane of WT cells, since HEK293 cells do not endogenously express the NK1 receptor (results not shown). Differences between the two constructs 
were clearly visible from the localization of the cameleon protein (Fig. 3B, E). The T2A construct releases the cameleon protein which accumulates in the cytoplasm. The T2ANC construct on the other hand fuses the cameleon protein to the cytoplasmic C-terminus of the membrane bound NK1 receptor, and as a result membrane co-localization of YC3.6 and $\mathrm{SP}^{\text {TAMRA }}$ on the membrane is visible in Fig. $3 \mathrm{~F}$, but not in $2 \mathrm{C}$.

Previous studies [67] have shown that binding of SP to NK1 results in activation of the downstream signalling pathway leading to a temporal rise in cytoplasmic calcium ion concentrations (section 3.5) and, also, irregular bulges of the plasma membrane known as membrane blebbing as clearly visible for both constructs in Fig. 3.

\section{Design of a resealable microfluidic flow cell}

We aimed to develop a biosensor capable of specifically measuring GPCR-activating analytes in a series of samples using fluorescence imaging. For best optical and chemical properties and easy handling, a resealable flow cell was designed composed of two borosilicate glass slides separated by a gasket of fluorinated elastomer which included a microfluidic inlet and outlet (Fig. 1). The slides were sealed together in a Fluidic Connect Pro flow cell holder (Micronit), which allowed immediate fluidic connection to a set of 3 syringe pumps that provided a constant flow of cell culture medium, and, using an injection valve $(5-100 \mu \mathrm{l}$ loop volume), seamless introduction of samples. Cells remained adherent to the poly-lysine modified surface at flow speeds below $400 \mu \mathrm{l} / \mathrm{min}$. The flow cell dimensions were $170 \mathrm{~mm}^{2}$ $x 0.3 \mathrm{~mm}$, but in principal the surface could be $800 \mathrm{x}$ smaller as we monitored an area of only $\sim 0.2 \mathrm{~mm}^{2}$ containing $\sim 50$ cells.

\section{Image acquisition and analysis}

After excitation with $458 \mathrm{~nm}$ a confocal laser microscope collected image emission spectra every 2 seconds for $512 \times 512$ pixels. This image series was automatically analysed by spectral unmixing on a pixel-by-pixel base to provide a value of the change in FRET (YFP/CFP ratio) resulting from the cytosolic calcium transients. For image processing we applied a custom made plugin named PRI-FRET on ImageJ (v1.46). This plugin can set lower and upper threshold values on the YFP and CFP images to remove background noise and saturated intensity pixels, and subsequently ratio and average relative intensities of all remaining dynamic YFP and CFP pixels over time. All ratio averages were subsequently normalized by subtraction of the averages of those ratios obtained before injection.

Without an internal reference to remove non-specific responses this biosensor would be a general sensor of $\left[\mathrm{Ca}^{2+}\right]$ changes inducing analytes, but not of a particular GPCR. As we only observe statically within a single flow channel, we introduced a method for automatic detection and subtraction of non-specific signals by mixing the GPCR cells 3:1 with red coloured reference cells expressing the red fluorescent protein mCherry instead of the receptor (Fig. 2A). We made use of the fact that mCherry has an excitation optimum of 543 

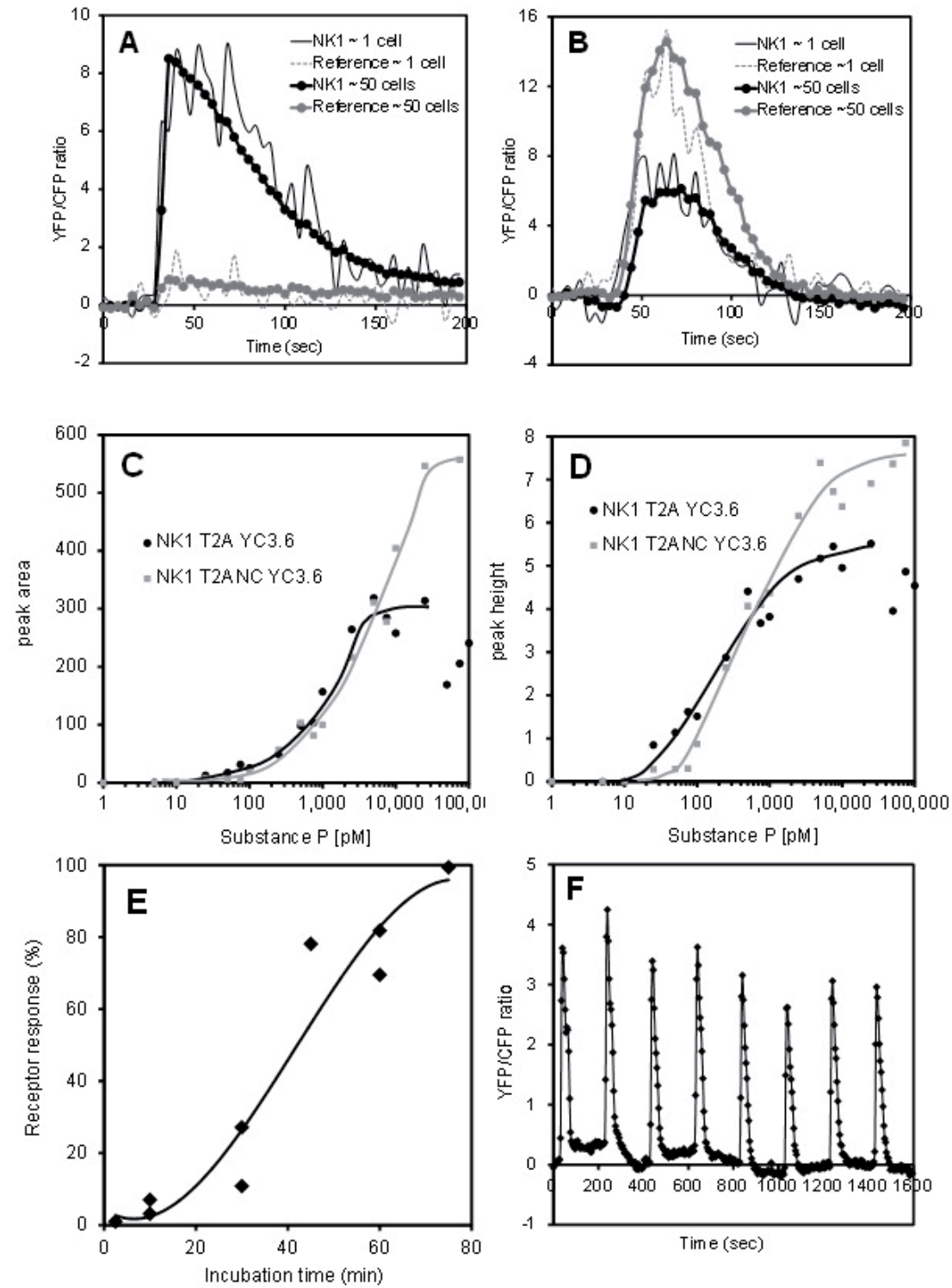

Figure 4, NK1-biosensor evaluation in terms of specificity, sensitivity, dose-response, time-torecovery, and re-usability. Cameleon FRET ratioing monitors $\left[\mathrm{Ca}^{2+}\right]$ changes in the mixed NK1 and $m$ Cherry reference cells. Response profiles of ca 50 mixed cells were imaged every 2 seconds in one field of view $\left(0.2 \mathrm{~mm}^{2}\right)$ while injecting $A, 10 \mathrm{nM} \mathrm{SP}$ and $B, 2 \mu \mathrm{M}$ ionomycin. Red mCherry cell pixels were graphically separated from the green NK1 cell pixels. Injection of the NK1-specific ligand SP yielded only a $\left[\mathrm{Ca}^{2+}\right]$ changes in NK1 expressing cell(s), and injection of the non-specific ionophore ionomycin yielded $\left[\mathrm{Ca}^{2+}\right]$ changes in both NK1 and reference cells. The signals for both 50 and single cells are given. Dose-response curves based the integrated peak area $\mathbf{C}$ and the maximum peak height $\mathbf{D}$, are plotted against the ligand concentration. In $\mathbf{E}$ the recovery time of the NK1 receptor response after a saturating injection of $50 \mathrm{nM}$ SP is shown. In $\mathbf{F}$ the response of NK1 cells to injections repeated every 200 seconds with lower $1 \mathrm{nM}$ concentrations of SP is shown. Images were taken every 4 seconds. All experiments $\mathbf{A}-\mathbf{F}$ were performed at a flow rate of $100 \mu \mathrm{l} / \mathrm{min}$ and with $50 \mu \mathrm{l} \mathrm{sample.}$ 
$\mathrm{nm}$, which does not excite the YFP or CFP proteins of YC3.6. Processing of images with a mix of receptor and reference cells was done by input of a reference cell image. Before or after the FRET time series a single mCherry image was made to determine which cameleon pixels were derived from reference cells. The software then generated separate normalized ratio curves for the receptor and reference cells.

\section{Specificity}

To test the GPCR biosensor specificity Fig. 4A and B show the results after injection of an NK1-specific ligand versus a non-specific ionophore for calcium ions across membranes. Plotted are the signal averages from single cells and $\sim 50$ cells in the same view. The receptor specific ligand SP (10 nM) increased FRET signals only in the receptor cells and not in mCherry cells (Fig. 4A). Injection of $2 \mu \mathrm{M}$ ionomycin (Free Acid, S. conglobatus, Calbiochem) resulted in responses in both NK1 receptor and mCherry reference cells. Thus this design allows that non-specific signals from mCherry cells can be deducted automatically from signals obtained from the GPCR cell fraction to ensure that the recorded responses are GPCR-specific.

\section{Sensitivity range}

To determine the lower and upper sensitivity range of the NK1 receptor, response curves were made for both the T2A and T2ANC receptor constructs of NK1. Injections of $50 \mu \mathrm{I}$ SP at concentrations ranging from $1 \mathrm{pM}$ to $100 \mathrm{nM}$ at a flow speed of $100 \mu \mathrm{l} / \mathrm{min}$ were performed to measure the activation of the NK1 receptor. At concentrations above $1 \mathrm{nM}$ SP a fresh slide was used for each measurement to avoid effects of receptor saturation from previous runs. Fig. $4 C$ and $D$ show the correlation of peak area and height of transient FRET ratio curves with SP concentration. For the NK1-T2A-YC3.6 clone the optimal SP concentration range (linear part of the S-curve) based on measured peak heights (Fig. 4D) was 25-2500 pM, whereas for peak area (Fig. 4C) it ranged from 100-5000 pM. For the NK1-T2A-NCYC3.6 clone the optimal SP concentration range based on peak height (Fig. 4D) was 1002500 pM and for peak area (Fig. 4C) it ranged from 250-25000 pM. In both dose response curves the T2ANC cell line reached higher maximum values. The cell lines were clonal lines, which meant that all cells express approximately the same number of receptor molecules on the membrane. The response differences may be explained by the possibility that clone NK1-T2ANC-YC3.6 has more or stronger expressed copies of the NK1 receptor gene, and, therefore, a higher density of receptors available on the surface compared to the NK1-T2AYC3.6 clone. Higher densities of receptors lead to stronger activation of the transient [ $\left.\mathrm{Ca}^{2+}\right]$ changes and higher maximum values in the dose-response curve.

\section{Measurement throughput and regeneration time}

Earlier studies describe that after activation, the NK1 receptor internalizes in endosomes followed by receptor dissociation from the ligand and receptor recycling back to the 
membrane, while the ligand is degraded internally [47]. As a result there is a transient drop in the number of available receptor sites. This phenomenon is especially relevant for a biosensor application in which cells are repeatedly exposed to potentially high concentrations of activating ligands which would saturate and remove available receptor sites from the membrane.

In Fig. 4E we show, that, while maintaining cells at RT and $100 \mu \mathrm{l} / \mathrm{min}$ continuous flow of fresh cell culture medium, full recovery of the FRET signal, after exposing the receptor to a saturating dose of $50 \mathrm{nM} \mathrm{SP}$, is a gradual process taking around 80 minutes to complete. In biosensor measurements knowledge of this recovery curve helps to interpret the reliability of subsequent measurements after the response has passed a certain threshold value.

To demonstrate the throughput of the biosensor to yield accurate responses to repeated injections of $1 \mathrm{nM} \mathrm{SP}$, an experiment was done repeating 8 injections every 200 seconds using the NK1-T2A-YC3.6 cell line (Fig. 4F). A slight fluctuation in peak height was observed, probably due to fact that data points are 4 seconds apart, thus missing the maximum peak height between two subsequent scans. A larger time interval between data points (4 seconds) was chosen to prevent photo bleaching of the YC3.6 fluorophore during continuous measurements for 30 minutes. The limited but apparent signal reduction of around $10 \%$ across the 8 measurements may reflect a reduction in receptor density due to the repeated activations at that concentration and frequency.

\section{Relationship of injected sample volume to flow cell volume}

Our test samples were injected via fused silica capillaries of $0.15 \mathrm{~mm}$ inner diameter $\left(0.018 \mathrm{~mm}^{2}\right)$ and released into a flow cell of cross section $0.3 \mathrm{~mm}$ height $\times 4 \mathrm{~mm}$ width $(1.2$ $\mathrm{mm}^{2}$ ). This 68-fold expansion in cross-section slows the linear flow speed, and increases the surface area over which diffusion can occur of sample analyte with media ahead and behind it. For the standard flow cell of $50 \mu$ volume we determined the peak shapes of both the analyte concentrations and the biosensor responses after injecting 5, 50 and $100 \mu \mathrm{l}$ of 1 $\mathrm{nM} \mathrm{SP}$ at a continuous flow of $100 \mu \mathrm{l} / \mathrm{min}$. The relative concentrations of analyte contacting the cells in the microscope field of view, were determined by mixing SP samples with an inert fluorescent dye as internal standard ( $1 \mu \mathrm{M}$ fluorescein). By taking injection volumes $2 \mathrm{x}$ larger and 10x smaller than the flow cell volume, we were able to characterize the changes in the shape of the sample concentration profile due to increased diffusion and laminar flow effects. Fig. 5 shows the profiles of the sample concentration and FRET ratio response after injection of $100 \mu \mathrm{l} \mathrm{SP}$ (Fig. 5A), $50 \mu \mathrm{l} \mathrm{SP}$ (Fig. 5B) or $5 \mu \mathrm{l} \mathrm{SP} \mathrm{(Fig.} \mathrm{5C).} \mathrm{Table} 1$ summarizes the results of Fig. 5 by giving the deviation from the expected value if there would be no diffusion or other dispersive effects. When no dispersive effects would take place, the fluorescein signal from the $5 \mu$ l loop would be expected to have the same peak height as the larger injected volumes with the same sample concentration, a duration of 3 seconds 
Table 1, Effects of sample volume in relation to flow cell volume $(50 \mu \mathrm{l})$ on the sample concentration (fluorescein) and FRET ratio response characteristicsa

\begin{tabular}{|c|c|c|c|c|c|}
\hline & $\begin{array}{l}\text { Injected } \\
\text { volume }\end{array}$ & $\begin{array}{c}\text { Fluorescein } \\
\text { characteristics }^{\mathrm{a}}\end{array}$ & $\begin{array}{c}\text { Deviation from } \\
\text { the expected } \\
\text { value }(\%)^{a}\end{array}$ & $\begin{array}{l}\text { FRET response } \\
\text { characteristics }^{\text {a }}\end{array}$ & $\begin{array}{c}\text { Deviation from } \\
\text { the expected } \\
\text { value }(\%)^{a}\end{array}$ \\
\hline \multirow[t]{3}{*}{ Peak height } & $100 \mu \mathrm{l}$ & $69 \mathrm{FU}$ & $0 \%$ & $9.9 \mathrm{RU}$ & $0 \%$ \\
\hline & $50 \mu \mathrm{l}$ & $68 \mathrm{FU}$ & $-2 \%$ & $9.6 \mathrm{RU}$ & $-3 \%$ \\
\hline & $5 \mu \mathrm{l}$ & $19 \mathrm{FU}$ & $-72 \%$ & $3.9 \mathrm{RU}$ & $-60 \%$ \\
\hline \multirow[t]{3}{*}{ FWHM $^{b}$} & $100 \mu \mathrm{l}$ & $64 \mathrm{sec}$ & $+6 \%$ & $21.5 \mathrm{sec}^{\mathrm{c}}$ & n.d. ${ }^{d}$ \\
\hline & $50 \mu l$ & $32 \mathrm{sec}$ & $+6 \%$ & $22.0 \mathrm{sec}$ & n.d. \\
\hline & $5 \mu \mathrm{l}$ & $13 \mathrm{sec}$ & $+332 \%$ & $10.5 \mathrm{sec}$ & n.d. \\
\hline \multirow[t]{3}{*}{ Peak area } & $100 \mu \mathrm{l}$ & $4388^{e}$ & $+6 \%$ & $356^{e}$ & n.d. ${ }^{d}$ \\
\hline & $50 \mu \mathrm{l}$ & 2280 & $+10 \%$ & 236 & n.d. \\
\hline & $5 \mu \mathrm{l}$ & 282 & $+36 \%$ & 47 & n.d. \\
\hline
\end{tabular}

\footnotetext{
a Substance $\mathrm{P}(1 \mathrm{nM})$ mixed with $1 \mu \mathrm{M}$ fluorescein as internal standard wasinjected into a flow cell of $50 \mu \mathrm{l}$ in volumes of 100,50 or $5 \mu \mathrm{l}$. The effect of substrate dilution is quantified relative to $100 \mu \mathrm{l}$ injected in the $50 \mu \mathrm{l}$ flow cell.

${ }^{\mathrm{b}}$ Full Width at Half Maximum (FWHM) measured in seconds

'The main calcium response peak, not including the right shoulder.

${ }^{\mathrm{d}}$ The deviation from the expected value for FRET signal areas was not determined since longer exposure times influence peak areas in a non-linear manner.

${ }^{\mathrm{e}}$ The peak area (FU or RU x sec) includes the peak shoulder.
}

(FWHM) and a peak area proportional to the injected quantity. Peak height, however, was $72 \%$ lower than the injected concentration value, and peak width over 4 times broader due to diffusion. Peak area was $128 \%$ the expected value, possibly due to slower speeds of the compound close to the flow cell surface where the cells are and measurements are taken. The results show that sample diffusion has pronounced effects on sample concentration when the sample volume is much smaller (10x) than the flow cell volume. Also the FRET ratio response signals deviated from the expected values. Peak height was $60 \%$ lower than the expected value in correspondence with the $72 \%$ lower sample concentration. Peak width, when including the shoulder peak, was shorter than sample exposure time at long exposures of 60 seconds (Fig. 5A), the same at sample exposures of 30 seconds (Fig. $5 \mathrm{~B}$ ), and longer at sample exposures of around 13 seconds (Fig. $5 \mathrm{C}$ ). This shows how these responses follow their own internal dynamics after the initial trigger. This is also visible from the two longer exposure times, which show a peak response plus an additional shoulder, which is the result of calcium oscillations [121]. The longer the receptor is exposed to the ligand, the more and the longer these oscillations occur. These peak shoulders are not very informative and the larger volumes passed through the sensor are potentially wasteful. We conclude, therefore, that the optimal sample volumes for GPCR-based cellular biosensors are equal to the volume of the biosensor flow cell and apply flow rates that allow the entire sample to be passed in less than 30 seconds. 


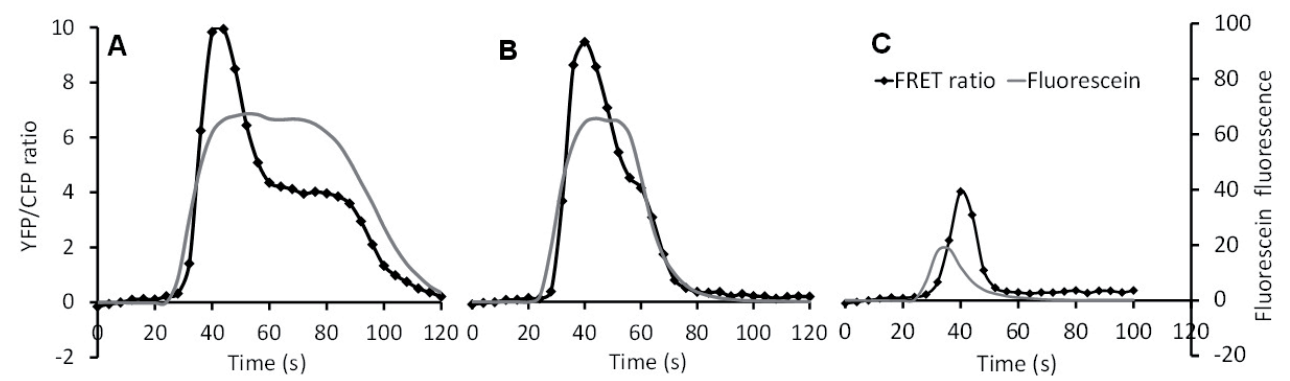

Figure 5, Activation of the NK1 receptor by different volumes of $1 \mathrm{nM} \mathrm{SP}$ using a fluorescent marker as internal standard of the ligand concentration. Injections were done of $100 \mu \mathrm{l}(\mathrm{A}), 50 \mu \mathrm{l}(\mathrm{B})$, and $5 \mu \mathrm{l}$ (C) of $1 \mathrm{nM}$ SP into a $50 \mu \mathrm{l}$ flow cell at a constant flow rate of $100 \mu \mathrm{l} / \mathrm{min}$. The YFP/CFP ratio is plotted on the left-axis axis and the fluorescein intensity on the right-axis. Fluorescein intensity $(1 \mu \mathrm{M})$ is measured in the background. Measurements were taken every 4 seconds.

\section{Conclusions}

The presented NK1 GPCR biosensor provides proof of principle for GPCR biosensors in general as systems with large potential for highly sensitive, flexible and quantitative microfluidic sensing devices. Unlocking the potential of utilizing the 800 human members of the GPCR gene family (and of genes of other organisms) for a broad array of biosensing needs in the medical, pharmaceutical, environmental, food and cosmetic domains promises to be an exciting area of research and development. The unique components of this GPCR biosensor device provide important potential advantages over other available detection systems.

1. Cameleon - Cameleon YC3.6 is a genetically encoded calcium indicator which allows immediate measurement of cytoplasmic calcium ions concentrations, independent of indicator concentration, due to ratiometric imaging. Other systems require loading of small molecule indicators, that might get sequestered or leak out over time compromising measurement accuracy. The use of this $\mathrm{GECl}$, furthermore reduces costs of chemicals and time, and improves reproducibility of measurements.

2. T2A for correlated concentrations of receptor and calcium indicator - The use of T2A or T2ANC allows a direct assessment of the receptor concentration and localization in any evaluated cell. Low expression of cameleon will be directly correlated to low expression of the receptor. This aids in the selection of the desired expression levels in clonal cell lines and provides an internal check that the receptor is expressed.

3. High content imaging - The flow cell is embedded in a chip holder, which provides a microscope viewing window, that currently allows the use of microscope objectives for detailed imaging with $20 x$ objectives. Other designs of the current commercially available chipholder could allow spatial access of objectives with shorter working 
distances and higher numerical apertures to obtain images with further increased optical resolution and thus provide information about subcellular processes.

4. Sensitivity - The sensitivity of ligand detection will typically depend on the $\mathrm{EC}_{50}$ for the chosen receptors. In our example with NK1 and SP we could detect ca. 30 pM SP in $50 \mu$ of sample corresponding to $2 \mathrm{pg}$ of analyte. However, the system operates on sample loop injections that are optimal when they approach the flow cell volume. The commercial flow cell we used was $\sim 50 \mu \mathrm{l}$ with a surface area of $170 \mathrm{~mm}^{2}$. Large reductions in flow cell volume are possible in the current design as the volume depends on the circumference of the gasket which is 850 times larger now than the monitored area of $0.2 \mathrm{~mm}^{2}$.

5. In- or at-line measurements - The procedure of injecting samples from an injection loop into the biosensor device can be easily automated and is potentially suitable for in- or at-line measurements. We estimate that a cell based biosensor needs to be replaced every 8 hours and is potentially capable of 100 measurements.

6. Data analysis - Images were processed based on an ImageJ plugin which can be automated to provide online digital graphic outputs of activities in samples.

7. Low cost detection - The system was operated on an advanced Zeiss confocal microscope using spectral unmixing. However, a normal fluorescence microscope could be used as well since YFP and CFP measurements are well possible using excitation and emission filters and even CFP measurements alone could provide a reliable output (although CFP concentration dependent). Furthermore, as signal intensity is more important for biosensors than localization confocality is not needed to measure signalling events.

The system also carries certain potential disadvantages:

1. Throughput - Even though the frequency of measurements could be raised to a sample every 3 minutes, these are sequential, and there are issues of fluorescence bleaching and receptor internalization which at high imaging frequencies and high doses affect subsequent measurements. A possible solution to this problem would be to use the current flow cell principle to design highly parallel flow cells which can be addressed sequentially and/or in parallel at optimal frequencies to ensure reliability and to improve throughput [140].

2. Handling and assembly must be done by skilled laboratory personnel - like maintaining cell cultures and operating a fluidic system and microscope. This is also true of microtiter plate based cell assays, but could be simplified if dedicated instruments will be developed.

3. Transcriptional fusions based on the T2A-peptide can also be a disadvantage Some receptors do not allow high expression in cell lines. Selecting low expressors will then also reduce the signal intensity from cameleon. In some cases these two proteins may therefore better be uncoupled. A stable cell line with high expression 
of cameleon could be used for transfection of receptor proteins using a different selectable marker, thus avoiding this potential issue.

4. In a situation that a sample contains mixed compounds which trigger cumulative specific and non-specific responses, the net difference between the GPCR and reference cell line responses may still contain information of the specific response. The visibility of this difference will however depend on the relative induced signal strength.

\section{Acknowledgements}

We thank Gerrit Polder from WUR-Biometris for designing Image J plugin PRI-FRET, JanWillem Borst from WUR Biochemistry for getting us started on FRET measurements of cameleon, Doug Golenbock, principal investigator, who submitted pcDNA3-CFP and pcDNA3-YFP to the Addgene plasmid bank, and Harrie Verhoeven and Joachim Goedhart for critically reading the manuscript. Financial support was obtained from Nano4Vitality program 2008-I, and from NanoNextNL, a micro- and nanotechnology consortium of the Government of the Netherlands and 130 partners. 

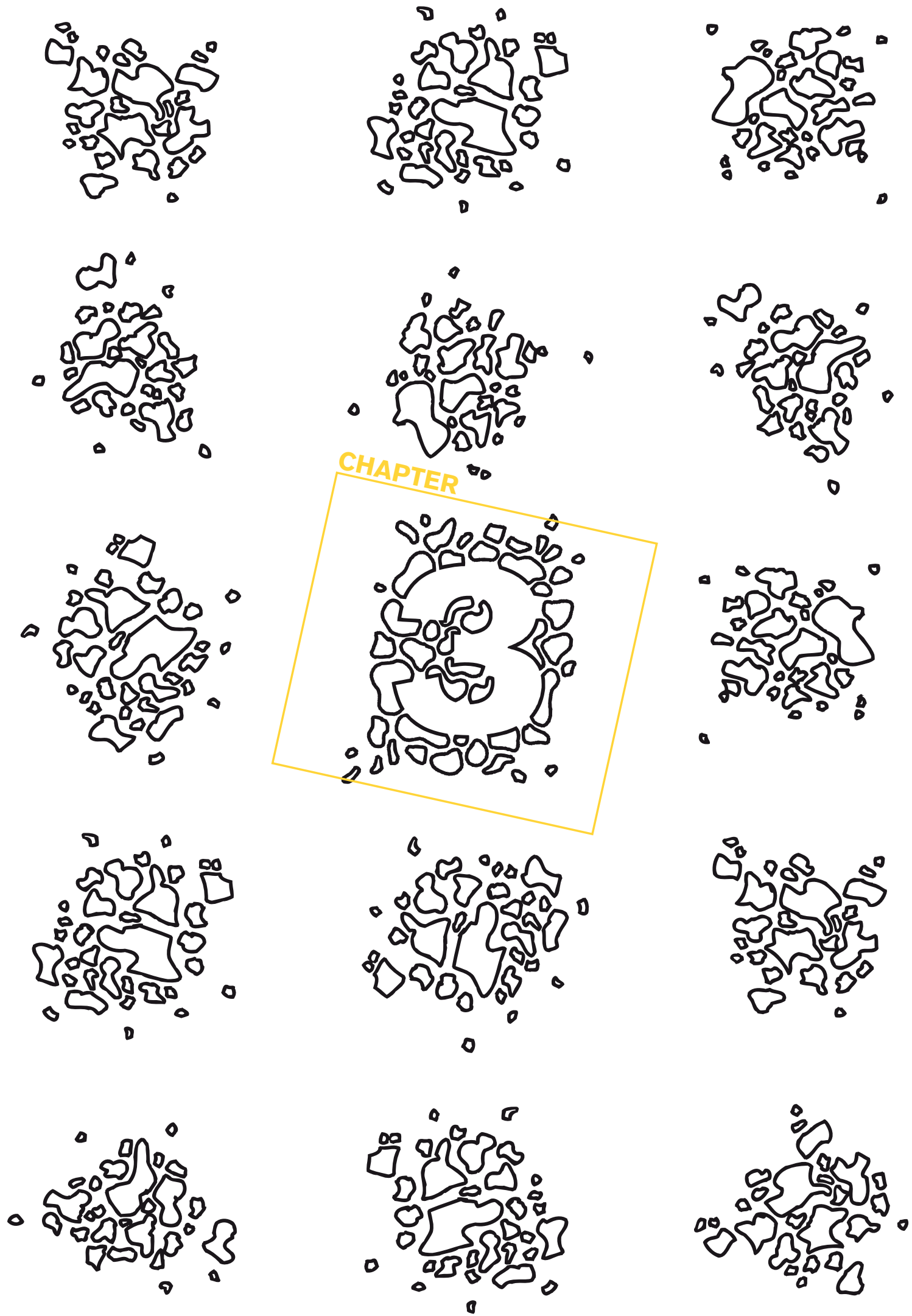


\section{Metabolomics meets}

\section{functional assays: coupling}

LC-MS and microfluidic

cell-based receptor-ligand

analyses

Maurice G.L. Henquet *, Margriet Roelse *, Ric C.H. de Vos, Bert Schipper, Gerrit Polder, Norbert C.A. de Ruijter, Robert D. Hall, and Maarten A. Jongsma

*These authors contributed equally to this article 


\begin{abstract}
Metabolomics has become a valuable tool in many research areas. However, generating metabolomics-based biochemical profiles without any related bioactivity is only of indirect value in understanding a biological process. Therefore, metabolomics research could greatly benefit from tools that directly determine the bioactivity of the detected compounds. We aimed to combine LC-MS metabolomics with a cell based receptor assay. This combination could increase the understanding of biological processes and may provide novel opportunities for functional metabolomics. We developed a flow through biosensor with human cells expressing both the TRPV1, a calcium ion channel which responds to capsaicin, and the fluorescent intracellular calcium ion reporter, YC3.6. We have analysed three contrasting Capsicum varieties. Two were selected with contrasting degrees of spiciness for characterization by HPLC coupled to high mass resolution MS. Subsequently, the biosensor was then used to link individual pepper compounds with TRPV1 activity. Among the compounds in the crude pepper fruit extracts, we confirmed capsaicin and also identified both nordihydrocapsaicin and dihydrocapsaicin as true agonists of the TRPV1 receptor. Furthermore, the biosensor was able to detect receptor activity in extracts of both Capsicum fruits as well as a commercial product. Sensitivity of the biosensor to this commercial product was similar to the sensory threshold of a human sensory panel. Our results demonstrate that the TRPV1 biosensor is suitable for detecting bioactive metabolites. Novel opportunities may lie in the development of a continuous functional assay, where the biosensor is directly coupled to the LC-MS.
\end{abstract}




\section{Introduction}

Metabolomics has become well-established as a valuable approach to investigate the metabolite profiles of living organisms. The technologies used are now firmly embedded in medical, microbial and plant research and are providing detailed snapshots of unique chemical fingerprints of biological responses. [141, 142]. We are however, still facing two significant technical and biological challenges. Firstly, determining the chemical identity (annotation) of the myriad of metabolites now detectable, but often never observed or studied before [143]. Secondly, determining their potential bioactivity. Generating metabolomics-based biochemical profiles without any associated assessment of the function of the extract or its biochemical component parts is only of indirect value in understanding a biological process [144]. This paper focuses on the latter aspect by coupling LC-MS mass identification with a flow through bioactivity screening on living cells.

Many metabolites interact with organisms at the cellular level through binding with membrane-bound receptors such as G-protein coupled receptors (GPCRs) and ligand gated ion channels (LGICs) $[145,146]$. Some of these receptors already have well-described specific ligand interactions. Many others however, are still poorly or even fully uncharacterized. In order to characterize individual receptors and find their equivalent ligands, a multiwell plate, cell-based end point assay is often the approach employed [147-150]. This screening process could be improved if the LC-MS analysis could be directly coupled to a continuous biological assay. For that, a flow assay format would be required to allow a dynamic monitoring of bioactivity in the course of an analysis and the direct association of a metabolite to a biological response. Here, we have chosen the transient receptor potential channel vanilloid 1 (TRPV1) ion channel as a model sensor. This ion channel has been wellstudied for its role in pain and heat perception. TRPV1 is a promiscuous cation channel, with a high preference for calcium, that can be activated by low $\mathrm{pH}$ as well as by physical factors such as heat $\left(>42^{\circ} \mathrm{C}\right)$ and membrane depolarization [151]. However, TRPV1 was first identified as a receptor for capsaicin; a pungent compound found in foods containing chili peppers.

We used capsaicin as standard to test the general properties of the TRPV1 biosensor which uses the intracellular calcium ion sensor, YC3.6, to detect the cytosolic calcium changes that are induced by the activation of the TRPV1 ion channel $[95,131]$. We tested the TRPV1 biosensor using fruit pericarp extracts from three contrasting Capsicum varieties. Two were selected with contrasting degrees of spiciness for characterization by HPLC coupled to high mass resolution MS. LC-MS fractions from one pepper extract were screened in a semi-continuous manner, to identify the different bioactive compounds. To confirm biological relevance and complete the proof of concept, we have performed an analysis of the sensation of pungency of tabasco by comparing results from the TRPV1 biosensor with a human taste panel. All findings are discussed in the context of the potential to use this microfluidic biosensor as the first significant step towards an online functional metabolomics tool. 


\section{Materials and Methods}

\section{Plasmid construction}

The TRPV1 receptor gene was obtained as a cDNA clone from GeneCopoeia (W1312) and cloned into PT2A-YC3.6 without a stop codon using the Ascl and EcoRI sites. The expression vector containing T2A [132], [133] and YC3.6 in a single reading frame and the control construct mCherry-T2A-YC3.6 have been previously described [95]. Plasmids containing single CFP (cyan fluorescent protein) and YFP (yellow fluorescent protein), pcDNA3CFP (Addgene plasmid 13030) and pcDNA3-YFP (Addgene plasmid 13033) were used for recording reference spectra.

\section{Cell culture and transfection}

HEK293H cells (ThermoFisher Scientific, 11631-017) were grown as a monolayer in DMEM (Dulbecco's modified eagle medium, Gibco, 21063-029) containing 1x non-essential amino acids (Gibco, 11140-035), 1x Penicillin-Streptomycin (Gibco, 15140-122) and 10\% FBS (Foetal bovine serum, Gibco, 26140). Cells were transfected with expression vectors PTRPV1-T2A-YC3.6 or pmCherry-T2A-YC3.6 using Effectene transfection reagent (Qiagen, 301425). Selection with Geneticin at $600 \mu \mathrm{g} / \mathrm{ml}$ (Gibco, 10131-027) was initiated $24 \mathrm{~h}$ after transfection and maintained for several weeks until stable insertion lines were obtained. From a mixed culture of insertion lines, based on mean fluorescence intensity, a suitable clonal line with intermediate YC3.6 expression was selected for all further experimentation.

\section{Pepper and tabasco extracts}

Three Capsicum lines from a large pepper genotype collection (nrs 12, 18 and 28, representing $C$. annuum, $C$. chinense and $C$. frutescens, respectively) were chosen for their reported contrasting levels of capsaicinoids [152]. Approximately $500 \mathrm{mg}$ frozen pepper pericarp (fruits excluding seeds stored at $-80^{\circ} \mathrm{C}$ ) per variety or $500 \mathrm{mg}$ tabasco suspension (a commercial water and vinegar extract of Capsicum frutescens var. tabasco) was diluted to $25 \%(\mathrm{w} / \mathrm{v}$ ) with $1.5 \mathrm{ml} 100 \%$ methanol. The samples were sonicated for 15 minutes, centrifuged, filtered through a $0.2 \mu \mathrm{M}$ PTFE membrane into an amber glass vial and stored at $-20^{\circ} \mathrm{C}$ prior to analysis. Aqueous-methanol extracts were analysed by HPLC (see 2.5 ) and directly diluted 1:300 or 1:3000 in buffer used in the biosensor assay.

\section{LC-MS-fractionation of pepper extracts}

Our standard LC-MS-based metabolomics profiling platform, composed of a HPLCPDA-LTQ-Orbitrap FTMS system (Thermo Scientific), was extended with a chip-based nano-electrospray ionization source/fractionation robot (NanoMate Triversa, Advion BioSciences), mounted between the PDA (photodiode array detector) outlet and the inlet of the LTQ-Orbitrap FTMS hybrid system [153]. The sample injection volume was $10 \mu \mathrm{l}$. For chromatographic separation a Luna C18 analytical column $(150 \times 2.0 \mathrm{~mm}, 3 \mu \mathrm{m}$ particle 
size; Phenomenex) was used with $\mathrm{H}_{2} \mathrm{O}$ and acetonitrile as carriers, both containing $0.1 \% \mathrm{v} / \mathrm{v}$ formic acid, at a flow rate of $190 \mu \mathrm{l} / \mathrm{min}$ and a column temperature of $40^{\circ} \mathrm{C}$. A linear gradient from 5 to $65 \%$ acetonitrile in MQ water was applied over a period of $45 \mathrm{~min}$, followed by 15 min washing and equilibration before the next injection. Isopropanol (at a flow of $30 \mu \mathrm{l} / \mathrm{min}$ using a syringe pump) was added to the LC eluent flow between the PDA and the NanoMate via a T-junction, to ensure a stable nanospray into the FTMS. The NanoMate source was operated in negative electrospray ionization mode with a HD_A_384 chip at a spray voltage of $1.7 \mathrm{kV}$. The NanoMate was used in the LC coupling mode with fraction collection. The total flow into the NanoMate $(220 \mu \mathrm{l} / \mathrm{min})$ was split using different capillary tubes for both MS spray $(0.480 \mu \mathrm{l} / \mathrm{min})$ and for 96 -wells plate fractionation $(219.5 \mu \mathrm{l} / \mathrm{min})$. LC-fractions were collected every $28 \mathrm{~s}$ (i.e., $102.6 \mu \mathrm{l}$ ) into a 96 wells plate (Twin tec, Eppendorf) cooled to $10^{\circ} \mathrm{C}$. After collection, the plate was quickly sealed and stored at $-20^{\circ} \mathrm{C}$ before further usage. The fractions were assayed on the TRPV1 biosensor as either pools or as separate 96 -wells fractions. To firstly create the 8 pools of 12 fractions, half of the well volume (51.3 $\mu l)$ was used. The pooled and individual fractions were freeze-dried, in order to remove the LC solvent, and then dissolved in $10 \mu \mathrm{I}$ DMSO (Dimethylsulfoxide). Since $10 \mu$ l extract was injected and only half of each fraction was re-dissolved in $10 \mu \mathrm{I}$ DMSO the sample was two times diluted compared to the original extract. Both pooled (8 pools of 12 fractions) and individual fractions were $200 x$ or $1000 x$ diluted in assay buffer, resulting in a 400x or 2000x dilution from the original extract, depending on their bioactivity.

\section{Quantification of capsaicinoids in tabasco}

Capsaicinoids in the tabasco product and pericarp extracts were quantified essentially as described for pepper pericarp [152], by injecting $10 \mu \mathrm{l}$ of the $75 \%$ methanol extract

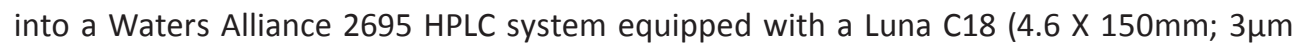
particles) at $40^{\circ} \mathrm{C}$ and a Waters 2996 PDA detector. A 30 min linear gradient of 50 to $75 \%$ acetonitrile in $\mathrm{MQ}$ water (both acidified with $0.1 \%$ formic acid) was applied to separate the capsaicinoids. The areas under the chromatographic peaks detected at $280 \mathrm{~nm}$ were used to calculate the concentrations of the individual and total capsaicinoids, using commercial standards (capsaicin and dihydrocapsaicin) from Sigma (St. Louis, USA).

\section{TRPV1-biosensor assay}

Assays using the TRPV1 cell lines were performed with a assay buffer of $100 \mu \mathrm{l} / \mathrm{min}$ DMEM medium containing $1 x$ non-essential amino acids, $1 x$ Penicillin-Streptomycin and $10 \%$ FBS. Extract and fraction samples, diluted in assay buffer, were injected with an injection loop volume of $50 \mu \mathrm{l}$ which is equal to the flowcell volume. Data series including the control cell line mCherry-T2A-YC3.6 were generated by mixing the two cell types at a 3:1 ratio of TRPV1:mCherry expressing cells. Both cell lines accurately reported receptor activations that induced subcellular cytoplasmic $\mathrm{Ca}^{2+}$ ion rise and fall. Imaging of Cameleon YC3.6 was performed using a Zeiss LSM 510-META 18 confocal laser scanning microscope as previously 
described in Roelse et al, 2013. Rapid simultaneous acquisition of CFP and YFP emission allowed the use of FRET (Förster resonance energy transfer) ratios as a monitor of ligand induced calcium dynamics. The ImageJ plugin PriFRET was used for the image analysis and pixel-based YFP/CFP ratio calculations according to the following equation:

$$
f(t)=\frac{\sum_{x>q}(y) / p}{\sum_{x>q}(c) / p}--\sum_{t=1}^{n} \frac{\sum_{x>q}(y) / p}{\sum_{x>q}(c) / p} / n
$$

Where: $f$ is the YFP/CFP ratio as a function of timestep $t ; y$ and $c$ are the pixel values of the YFP and CFP images respectively. One of the images YFP or CFP, selected by the user, is thresholded, taking only values larger than $q$ into account. The threshold value can be set by the user or calculated automatically, where it optimizes for a maximum $f(t)$ ratio. The function is normalized by subtracting the average YFP/CFP ratio over the first $n$ time steps. For the experiments described in this paper the threshold $q$ was set at zero and the normalization steps $n$ at 5. For each experiment, the first injection was done after a minimum of 5 images. The PriFRET ImageJ plugin is publically available at the WageningenUR website; http://www.wageningenur.nl/en/show/prifret.htm

\section{Human sensory panel evaluation}

A sensory panel experiment using tabasco was performed by the taste research company "Centrum voor Smaak onderzoek B.V." in Wageningen, The Netherlands. A panel of 41 humans were given a series of 6 samples, representing a dilution series of 5 samples from the pure tabasco product and 1 blank control without tabasco. Dilutions of tabasco were prepared in bottles of $1.5 \mathrm{~L}$ commercial mineral water (Spa Blauw). To prevent visual colour and density differences between the tabasco dilutions, $7 \mathrm{~g}$ of tomato sauce (Grand Italia Soffritto traditionale) was added to each $1.5 \mathrm{~L}$ mineral water. The panel members were given the tabasco dilutions in random order and asked to score the percentage perceived pungency. Individual scoring ranges were first normalized so that the highest and lowest score of each individual would have the same value. All intermediate scores were recalculated proportionally to maintain the relative differences. An ANOVA analysis was performed to determine significance of perceived taste differences between subsequent tabasco dilutions and results were tested for reliability using the Statistical Package for the Social Sciences software (SPSS version 15.0).

\section{Results and discussion}

\section{Metabolomics and functional assays on pepper extracts}

We first analysed pericarp extracts of three different Capsicum lines (lines 12, 18 and 28) that were chosen for their contrasting capsaicinoid levels. These three pepper lines were 

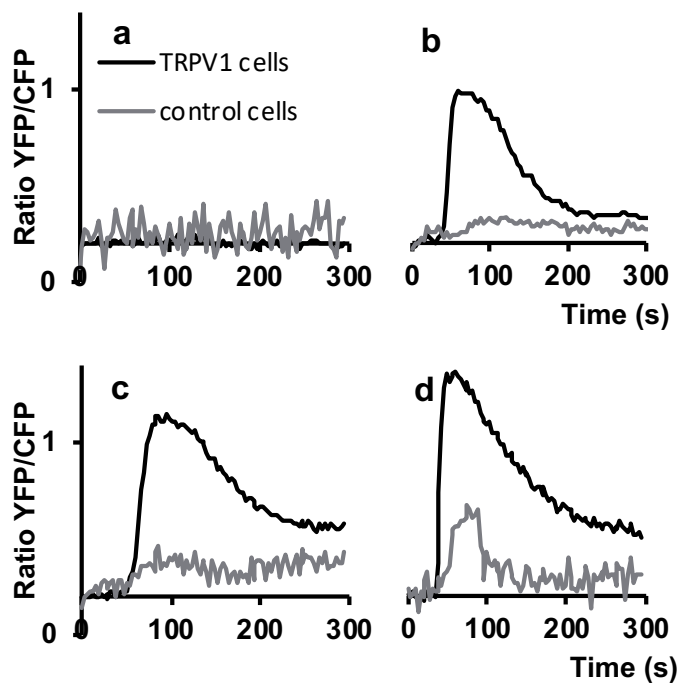

Figure 1, TRPV1 and control cell responses to crude pepper extracts. Cameleon calcium sensor YFP/CFP ratio changes in HEK239 cells were measured after injection of pericarp extracts from three different pepper lines with or without capsaicin spikes. The flow-cell contained both TRPV1expressing cells (black traces) and control cells (grey traces). a Line 12, 300X diluted; $\mathbf{b}$ line 12, 600X diluted and spiked with $50 \mathrm{nM}$ capsaicin; c line 18, 300X diluted; $\mathbf{d}$ line 28, 3000X diluted.

analysed for capsaicinoids by targeted HPLC-PDA (Table 1) and their levels corresponded well to previously determined levels [152].

Functional assays of these pepper extracts were subsequently performed with the TRPV1 biosensor. Figure 1 shows the TRPV1 and control cell responses to these pepper extracts. Line 12 showed no response at a 300x dilution of the extract (Figure 1a), corresponding to the HPLC analysis indicating the undetectable levels of both capsaicin and dihydrocapsaicin (Table 1). When line 12 extract was spiked with $50 \mathrm{nM}$ capsaicin, a normal TRPV1 cell response was observed, showing that the previously observed lack of response is not due to a masking effect by this line 12 (Figure 1b). In contrast to line 12, line 18 induced a clear response in the TRPV1 cells when exposed to a 300x dilution of the aqueous-methanol extract (Figure 1c) representing a final concentration of $24 \mathrm{nM}$ total capsaicinoids in the bioassay (Table 1). No response was observed with a $3000 x$ dilution of line 18 , representing $2.4 \mathrm{nM}$ of capsaicinoids (data not shown). The most pungent accession, line 28 (Figure $1 \mathrm{~d}$ ), which contained an approximately $110 x$ higher level of capsaicinoids compared to line 18 (Table 1), gave the highest signal even for the highest dilution tested (3000x). At this dilution, representing $274 \mathrm{nM}$ capsaicinoids, a brief, relative minor response in control cells lacking the exogenous TRPV1 receptor was observed (Fig 1d). This calcium response in control cells indicates the activation of an endogenous receptor to either the relative high capsaicinoids concentrations or an unknown component only present in the 3000x diluted Capsicum line 28 extract. Such responses from control cells were not observed in the $300 x$ diluted extract 
Table 1, HPLC quantification of capsaicinoids in crude extracts of three varieties of red chili peppers and a tabasco extract

\begin{tabular}{lccc}
\hline & $\begin{array}{c}\text { Capsaicin }(\mathbf{m g} / \mathbf{1 0 0} \mathrm{g} \\
\mathrm{FW})\end{array}$ & $\begin{array}{c}\text { Dihydrocapsaicin } \\
(\mathbf{m g} / \mathbf{1 0 0} \mathrm{g} \mathrm{FW})\end{array}$ & $\begin{array}{c}\text { Capsaicinoids in extract } \\
(\boldsymbol{\mu M})^{\mathrm{a}}\end{array}$ \\
\hline Line 12 & n.d. & n.d. & n.d. \\
Line 18 & 0.9 & n.d. & 7.2 \\
Line 28 & 75.3 & 25.3 & 821.8 \\
Tabasco & 14.4 & 9.2 & 192.6 \\
\hline
\end{tabular}

FW fresh weight, $n . d$. not detectable ( $<0.1 \mathrm{mg} / 100 \mathrm{~g} \mathrm{FW}$ )

${ }^{a}$ sum of capsaicin and dihydrocapsaicin the crude extract

of line 18, containing $24 \mathrm{nM}$ capsaicinoids. Therefore the control cell response is most likely caused by the high capsaicinoid concentration of line 28 .

The specific differential responses of the TRPV1 biosensor to the various Capsicum extracts at appropriate dilutions demonstrate that the microfluidic assay can be used on methanol plant extracts dissolved in cell culture medium without a need for further sample clean-up or compound purification. Furthermore, there appears to be a direct and (semi) quantitative relationship between the TRPV1 biosensor response, detected as a change in YFP/CFP fluorescence ratio, and the concentration of ligands present in the extracts (in this case to capsaicinoids), as analytically quantified by HPLC-PDA. This quantitative relationship is shown for pure capsaicin in section 3.3 of the results.

\section{Metabolomics and functional assays on pepper compounds}

The pericarp extracts of the two most contrasting pepper lines 12 and 28 were subjected to HPLC-PDA coupled to both chip-based nano-ESI with accurate mass Orbitrap FTMS and fractionation into 96 wells plates. The overlaid LC-MS chromatograms showed clear differences between these two red pepper lines in their metabolite profiles (Figure 2a, upper panel). As it was not the aim of the present study to determine all differentially accumulating compounds using comprehensive comparative metabolomics approaches, only the most obvious peaks were specified (Table 2), using the annotations previously reported by [154]. In general, line 28 , which showed highest activity in the TRPV1 bioassay, contained relatively high levels of capsaicinoids, while the TRPV1-inactive line 12, contained relatively high levels of quercetin-type flavonoids.

To identify which specific compounds in pepper line 28 interact with the TRPV1 ion channel, 96 LC-fractions each of $28 \mathrm{sec}$ of elution time were collected in a micro-well plate, using a TriVersa Nanomate fractionation robot, to be tested for their bioactivity. Initially, 8 pools $(\mathrm{A}-\mathrm{H})$, each containing 12 consecutive LC-fractions, were semi-continuously tested in the TRPV1 biosensor. The results in Figure 2a (lower panel) indicate a significant TRPV1-specific response, i.e. a clear signal above the baseline drift of the control cells, for only pools $\mathrm{G}$ and $H$. Subsequently, we analysed the individual fractions from these pools $G$ and $H$, at $400 x$ or 


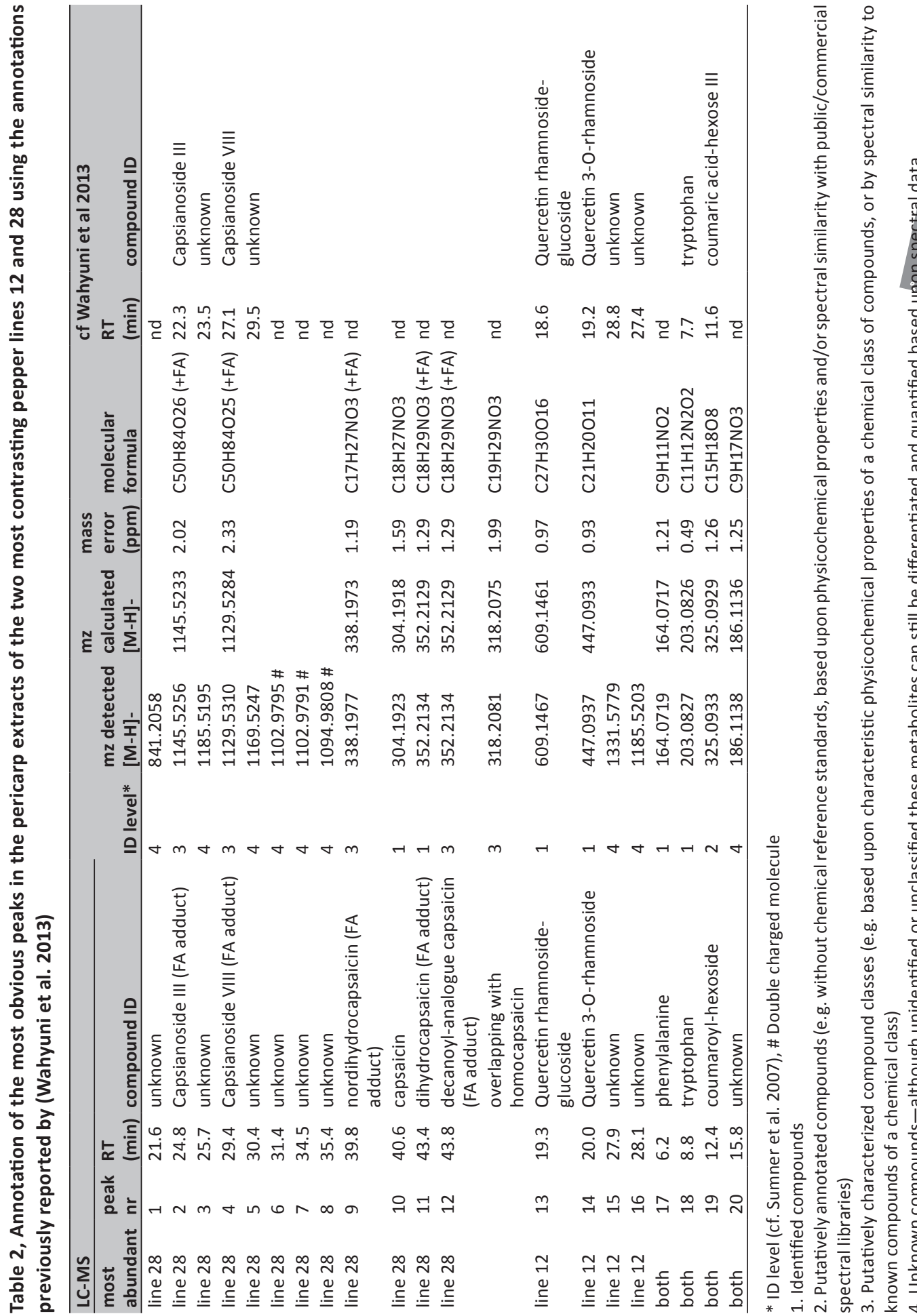



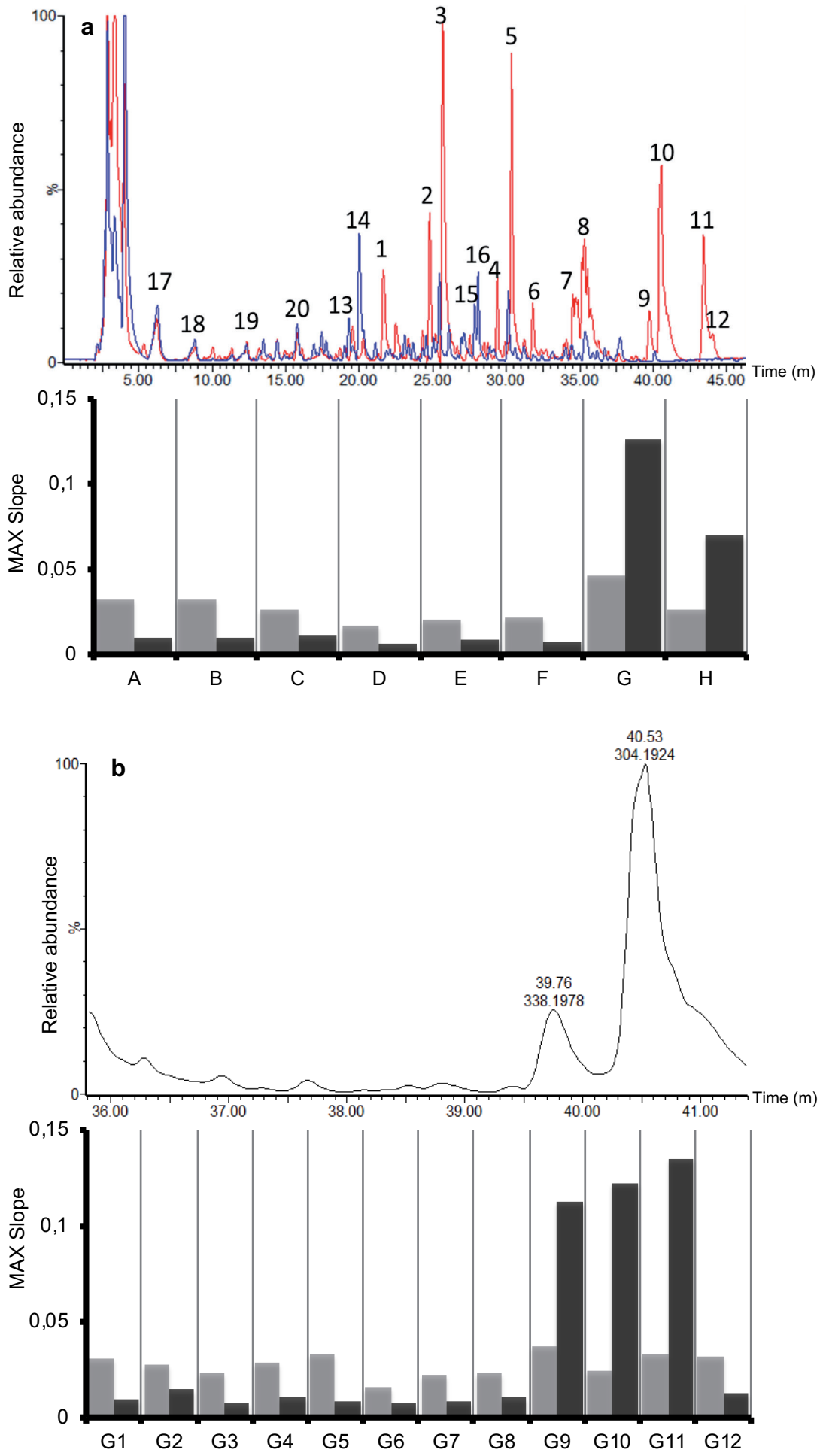

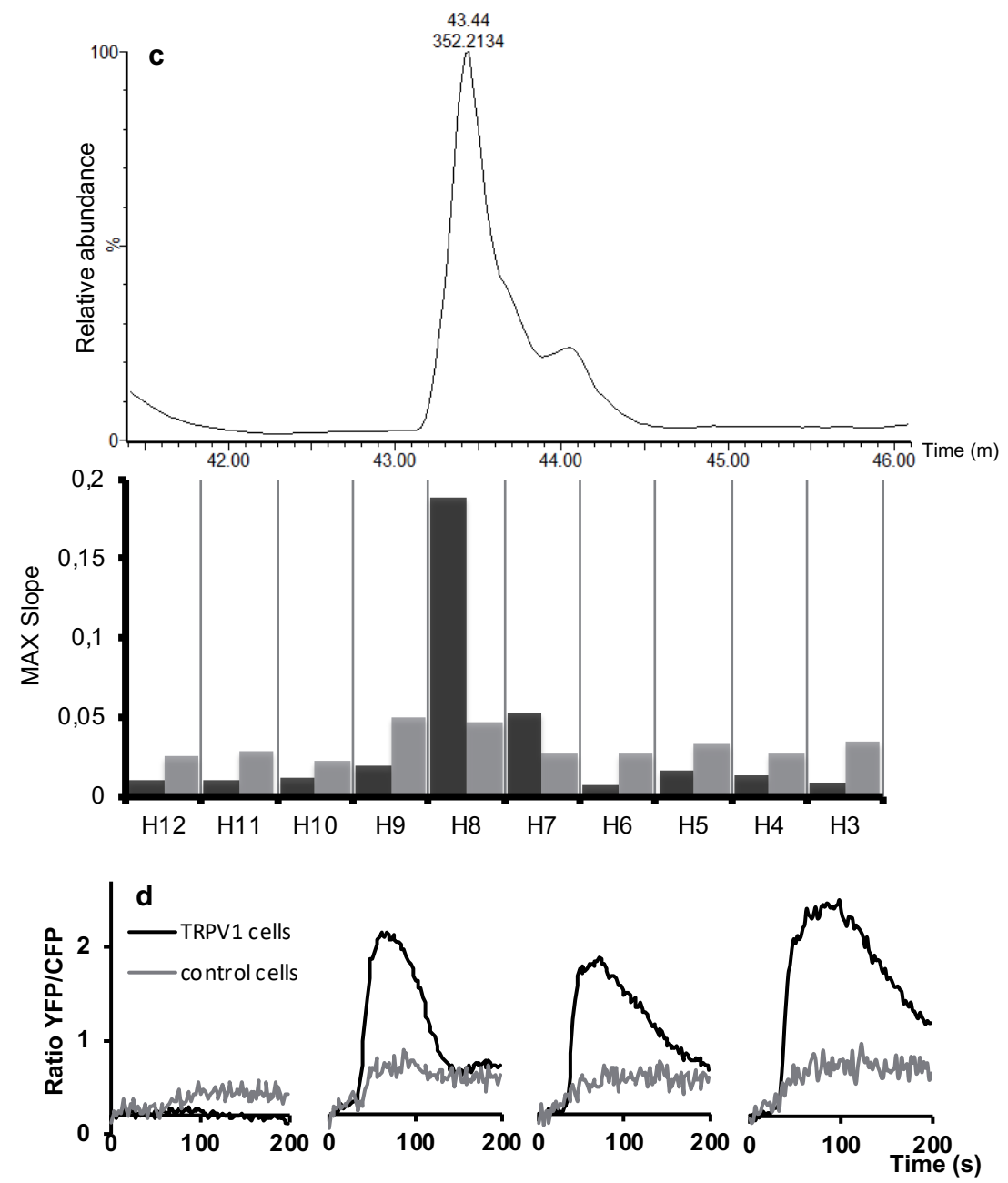

Figure 2, TRPV1 and control cell responses to LC-MS fractions of line 28. a, Overlaid LC-MS chromatograms (ESI neg mode; base peak intensity) of lines 28 (red) and 12 (blue). Chromatograms are at the same $\mathrm{Y}$-scale to enable direct comparison of the metabolite profiles of both pepper lines. The highest peaks were labelled (1-20) and annotated (Table 2). The NanoMate fractions obtained during LC-MS analysis were subsequently pooled in eight pools of 12 fractions as indicated with A-H. Responses (maximum slope from moving average of five measurements) to the pooled fractions are displayed below the chromatograms: TRPV1 in black and control in grey bars. b, and c, Details of LC-MS chromatogram and TRPV1 responses of line 28 corresponding to the individual fractions of bioactive pools $\mathrm{G}$ b and $\mathrm{H}$ c. All LC fractions were diluted 400X, except fractions G10 and G11 (coded with asterisk) which were diluted $2000 \mathrm{X}$, as compared to the original pericarp extract. d, Cameleon calcium sensor YFP/CFP ratio curves; from left to right: individual fractions G8, G9, G10 and G11 at 400X dilution (G8 and G9) and 2000X dilution (G10 and G11) 
2000x dilution of the original pericarp extract. The TRPV1 cells responded to the individual fractions G9, G10, G11, H7 and H8 (Figure $2 \mathrm{~b}$ and c). The control cells showed a relatively high calcium response with fraction G9, G10 and G11, this control response is caused by the high concentrations of capsaicinoids in these fractions. (Figures $2 b$ and d). In all cases, TRPV1 responding LC-fractions contained compounds detectable by their UV absorbance at $280 \mathrm{~nm}$, indicating the presence of an aromatic (phenolic) backbone. Analysis of the accurate mass data associated with these bioactive LC-MS peaks revealed several known pepper capsaicinoids, including nordihydrocapsaicin, capsaicin, dihydrocapsaicin and homocapsaicin, as well as less, known capsaicin derivatives (Supplementary Table 1). To our knowledge, we have shown that both nordihydrocapsaicin and dihydrocapsaicinare are also true agonists of the human TRPV1 ion channel, in addition to capsaicin.

\section{Dynamics of the TRPV1 biosensor}

The performance of the biosensor was characterized in more detail by measuring its response to different capsaicin concentrations in the sensor. Each capsaicin concentration was tested twice. Figure 3a shows the TRPV1 responses of the calcium indicator, expressed as the normalized YFP/CFP ratio, to a range of capsaicin concentrations. These data demonstrate that, in our flowcell system, HEK293 cells expressing TRPV1 are able to detect capsaicin in a dose-dependent manner within the concentration range of 5-500 nM. Concentrations above $500 \mathrm{nM}$ were not assayed because these may lead to desensitization of the TRPV1 ion channel $[155,156]$. To better visualize the dynamics of the system, the exposure time -or dose time- of 30 seconds is shown as a transparent grey area (Figure 3a). Related to this dose time we observed four response features: 1) The rate of response, or slope, appeared to be dose dependent. High concentrations of capsaicin showed a fast rise, while low concentrations showed a slower rise. 2) The response duration also appeared to be dose dependent: high concentrations showed a slow return to baseline levels, while low concentrations showed a faster return. 3) At the two highest capsaicin concentrations tested, 250 and $500 \mathrm{nM}$, the fluorescence ratios remained their maximum values for about 30 and 50 seconds, respectively, even after the ligand solution had passed through the flow cell. We call this an elongated response maximum. 4) In contrast, low concentrations of capsaicin, $<50 \mathrm{nM}$, showed a delayed response which only reached its maximum at 50 to 80 seconds, i.e. at least 20 seconds after the ligand solution had already passed through. We call this a delayed response maximum. These observed differences in dynamics give us greater insights into the receptor-ligand interactions at different ligand concentrations and hence represents a significant advantage of such a microfluidic system as compared to the currently used, multi-well, endpoint assay.

Differences in response dynamics of TRPV1 may be explained by existing models for TRPV1 binding stages [15, 157-159]. These models describe partial and full binding stages for capsaicin which affect the opening of the ion channel to partial and full opening, respectively. These binding stages of the TRPV1 ion channel are an effect of the ligand 

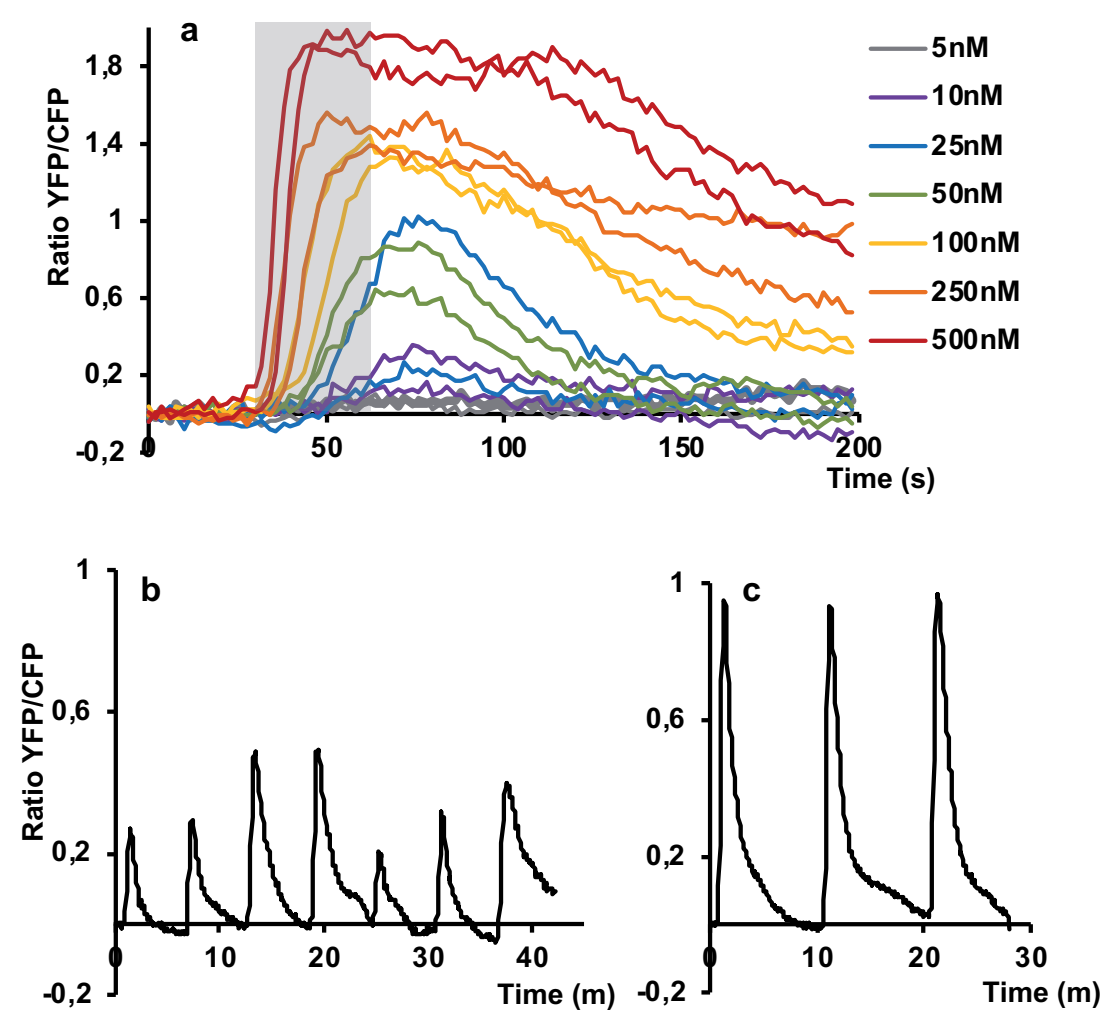

Figure 3, TRPV1 response to capsaicin. a, Cameleon calcium sensor YFP/CFP ratio changes upon injection of increasing capsaicin concentrations. Cell were exposed to 5-500 nM capsaicin for 30 $\mathrm{s}$ (indicated by the grey area) and their response followed up to $200 \mathrm{~s}$ after injection. $\mathbf{b}$, Cameleon calcium sensor YFP/CFP ratio changes upon repeated exposure to $30 \mathrm{nM}$ capsaicin, by injecting seven times at intervals of 6 min. c, YFP/CFP ratio changes upon $50 \mathrm{nM}$ doses injected three times at intervals of $10 \mathrm{~min}$.

binding to the ligand specific binding sites, of which there are at least two according to Hui et al 2003. At "high" concentrations of capsaicin, all binding sites on the TRPV1 receptor are occupied resulting in a full opening of the ion channel, while at "low" concentrations there is only a partial occupation of the available binding sites and therefore only a partial ion channel opening. The results we observed in the differential response dynamics of our TRPV1 biosensor at the various ligand concentrations (Fig 3a) fit well with such model.

Two experiments with repeated capsaicin injections were performed to obtain some insight into TRPV1 response and stability upon repeated exposure to ligands: I) 7 times 30 $\mathrm{nM}$ capsaicin at 6 min intervals and II) 3 times injections with $50 \mathrm{nM}$ capsaicin at $10 \mathrm{~min}$ intervals. 

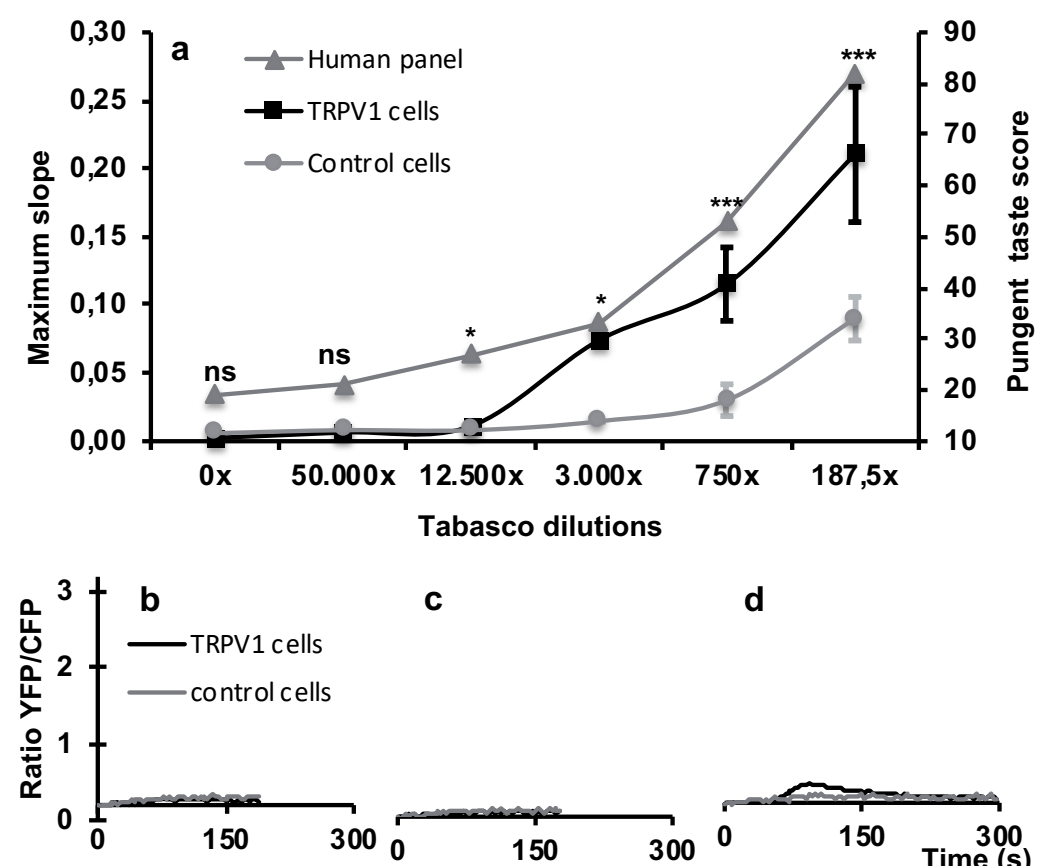

C

d
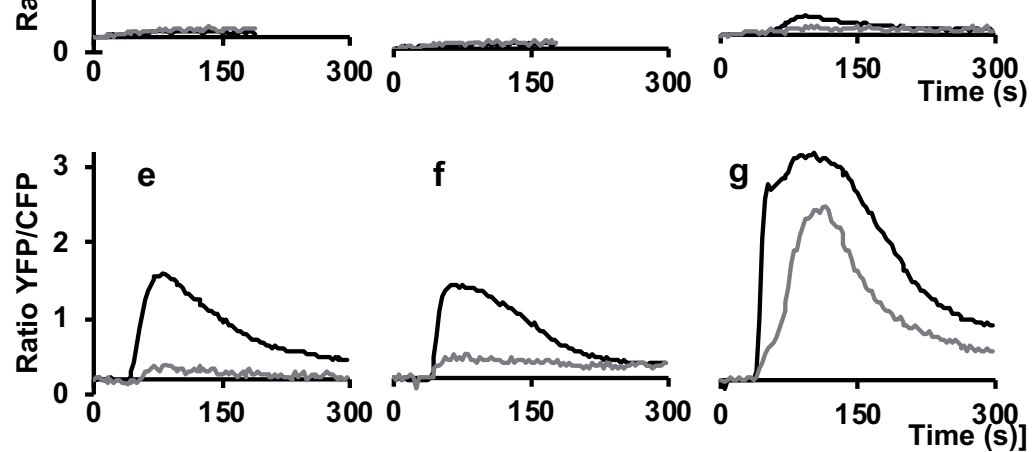

Figure 4, TRPV1 and taste panel responses to tabasco dilutions. a, benchmarking of the TRPV1 biosensor with human test panel results for detecting pungency of tabasco samples. The maximum increase rate of the Cameleon YFP/CFP ratio changes in the biosensor is plotted on the left axis (black squares TRPV1 cells; grey circles control cells). The result of the taste panel assay (grey triangles) is plotted on the right axis as a pungency score from 0 to 100 ; ns not significant, ${ }^{*}=p$ B 0.05 ; $^{* *}$ $=\mathrm{p} \mathrm{B} 0.01 ;{ }^{* *}=\mathrm{p} \mathrm{B} 0.001 . \mathrm{b}-\mathrm{g}$, Cameleon calcium sensor YFP/CFP ratio changes upon increasing tabasco concentrations (water control, 50.000X, 12.000X, 3.000X, 750X and 187.5X for plot $\mathbf{b}-\mathbf{g}$, respectively) in TRPV1 expressing cells (black curves) and control cells (grey curves).

At the relative lower concentration and intervals (Figure $3 \mathrm{~b}$ ), the response tended to vary considerably (standard deviation of 0.103 ), but without an obvious decline in sensitivity. In contrast, in the second experiment with the longer interval, and higher ligand concentration (Figure $3 \mathrm{c}$ ), we observed a highly reproducible pattern with signals fully returning to baseline values and with successive response levels equivalent to the previous ones (standard deviation of 0.014 ). The relatively high variation at lower concentrations may be due either to the shorter recovery periods ( 6 minutes) or may reflect the lower consistency 
in response at a ligand concentration close to the detection limit (as is also evident in Figure 3a). Nevertheless, while more detailed and dedicated experiments are required related to the injection frequency, uniformity and stability of the biosensor response, it is clear that this system has potential for sequential use in semi-quantitative / qualitative bioactivity screens.

\section{Biosensor response benchmarked against a human sensory panel}

To allow direct prediction of analysed plant samples to human taste, it is necessary to compare the TRPV1 biosensor system with in vivo taste perception by human individuals. For this reason, the TRPV1 biosensor was benchmarked against a human taste panel using a dilution series (187-50.000x) of a commercial tabasco sauce sample (Figure $4 \mathrm{a}$ and b) which had also been analysed for its capsaicinoid content. The most diluted tabasco sample was indistinguishable from the blank control by both the sensory panel and the biosensor assay. The tabasco pepper has a Scorville scale score ranging from 30.000 to $70.000 \mathrm{SHU}$, meaning that a dilution in this order of magnitude does not evoke a hot or pungent sensation. The taste panel however, was able to recognize the second-most diluted $(12.500 \mathrm{x})$ tabasco sample, just as the biosensor assay was able to detect a small signal for this dilution. This dilution corresponded to a capsaicin concentration of $\sim 15 \mathrm{nM}$ (Table 1). Higher concentrations (lower dilutions) gave increasingly large, responses from the panel as well as in the biosensor assay. With the two highest concentrations in the biosensor assay (equivalent to 250 and $\sim 1000 \mathrm{nM}$ capsaicin, respectively), the control cells also showed a response, likely caused by either too high concentrations of capsaicinoids or a native response to other compounds present in the tabasco. These results emphasise the importance of using control cells for determining ligand or matrix-induced a specific reactions and the use of the appropriate dilution series for more quantitative analyses of specific bioactivities. Interestingly, the sensitivity thresholds of in vivo (taste panel) and in vitro (receptor microfluidics) measurements were essentially the same, so that these in vitro results can be extrapolated directly to predict human sensory experience.

\section{Potential for online functional metabolomics}

The results presented were designed to provide a starting point for combining LC-MS analysis with bioactivity assays in series. Existing, online biochemical assays for anti-oxidant detection linked to LC-MS based component identification have already demonstrated the advantages of such online methods [160-162]. Our study provides the proof of concept that a microfluidic assay with living cells using analytical-scale LC-MS fractions can deliver detectable signals that allow the identification of bioactive molecules present in (crude) plant extracts. The next technical challenge is to design a truly continuous online functional metabolomics application in a flow through system using living cells as the biosensor. A continuous flowcell assay could allow direct measurement of LC output in a single experiment without the need for clean-up / extraction / re-dissolving steps which can 


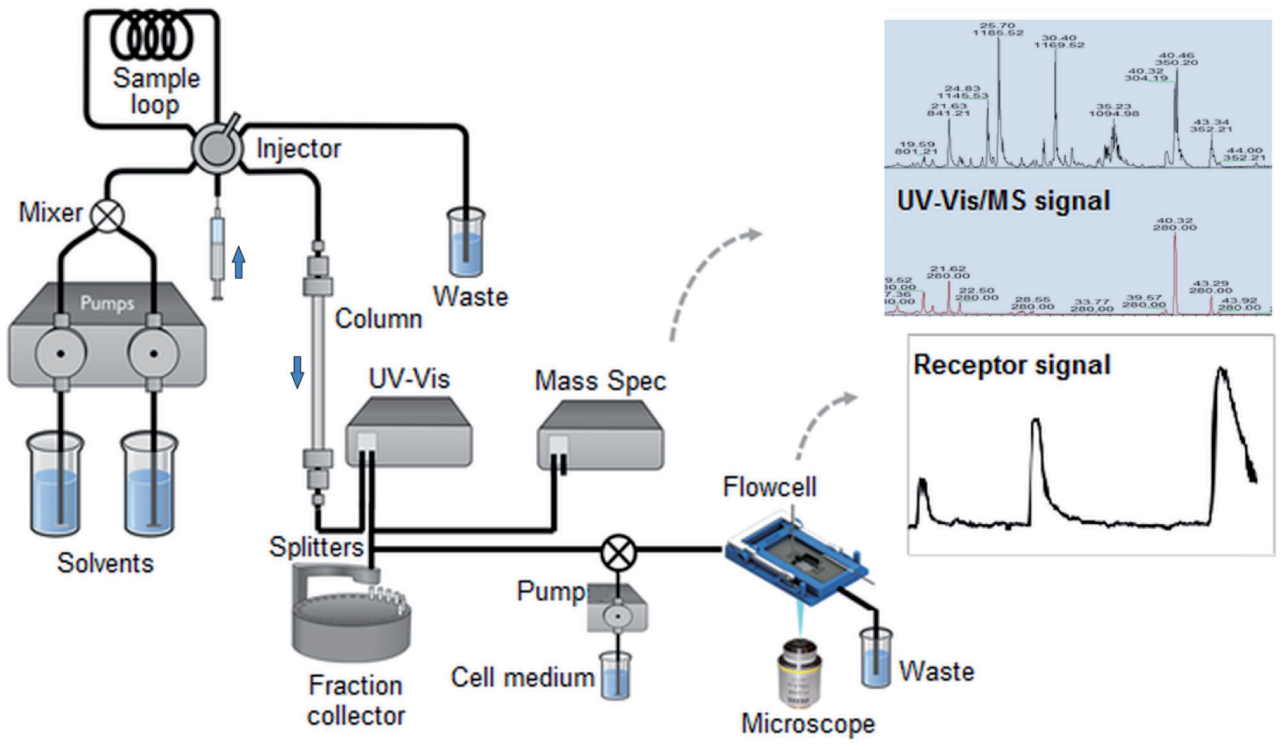

Figure 5, Scheme of an online functional metabolomics setup. The microfluidic flowcell with sensory cells is placed in a microscopic fluorescence detector, which will continuously monitor the cellular response of sample compounds eluting from the LC column. Simultaneous detection of compounds by UV-Vis lower chromatogram) and MS (upper chromatogram) can provide identification of bioactive compounds. LC-fractions, preferably separated into individual compounds, are collected as well in order to keep a compound record of the run when further functional analysis or metabolite identification experiments are required. This figure is modified from www.galenica.cl

lead to compound degradation or modification. Additionally, measuring in a flow-through system readily allows the direct study of receptor dynamics. Figure 5 shows a schematic representation of how such an online LC-MS-based metabolomics-biosensor platform could be realized.

There are, however, some key issues that still need to be addressed to realize such an online biosensor platform. Firstly, there is the issue that the LC solvent maybe incompatible with cell measurements. Acetonitrile or methanol, formic acid, low or high $\mathrm{pH}$, and deviant osmolality are toxic to cells. However, there are several ways to deal with this. Toxicity may be reduced or avoided by significant post column dilution of the sample, prior to cell exposure. Such a dilution is realistic, the plant LC fractions in this study could be diluted with cell culture medium up to 2000 times and still give a clearly measurable sensor response. Alternatively, detoxification of the running buffer may also be achieved by techniques like online post-column solvent evaporation, such as recently developed by [163]. Here the solvent leaving the analytical LC column is evaporated online and the compounds subsequently re-dissolved online in deuterated solvent needed for the NMR analysis.

A second issue is the long-term cell viability and the potential for repeated re-activation of the biosensor cells. In our study with the TRPV1 expressing HEK293 cells, the cells remained vital for at least 1 hour at room temperature while continuously imaging the receptor 
activation. Repeated dosage of up to $50 \mathrm{nM}$ capsaicin produced reproducible activation peaks. At higher dosages of capsaicin, the sensor can easily become saturated. Sample concentration should therefore be carefully monitored and adjusted to avoid receptor over exposure. These are key aspects that need further optimization in the current biosensor setup. However, an investment in time and effort is highly justified as online functional metabolomics using a cell based assay will provide valuable opportunities in automation / high throughput biochemical and bioactivity screening.

\section{Concluding remarks}

Here we have shown that a flowcell containing the HEK239 cell line expressing the TRPV1 ion channel coupled to intracellular calcium sensing, in combination with an appropriate control cell line, provides a sensitive method for semi-continuous functional metabolomics. TRPV1 was activated, resulting in dynamic intracellular calcium levels, both when cells are stimulated with the pure ligand, capsaicin, as well as with crude extracts of capsaicinoidcontaining Capsicum accessions. Additionally, we have shown that this biosensor compares in sensitivity to a human sensory panel. Furthermore, through application of this system we have been able to determine that, next to the well-known ligand capsaicin, nordihydrocapsaicin and dihydrocapsaicin are true agonists of the human TRPV1 ion channel in hot pepper. A next step will be to tackle the next technical challenges, and meet all biological demands needed to convert this system into a truly continuous and high throughput online functional metabolomics tool.

\section{Acknowledgments}

We acknowledge the Centrum voor Smaakonderzoek (CSO) for performing the taste panel study. This work is supported by NanoNextNL, a collaborative project of the Dutch government and 130 partners, and by the Netherlands Metabolomics Centre, which is part of the Netherlands Genomics Initiative / Netherlands Organisation for Scientific Research.

\section{Compliance with ethical standards}

\section{Conflict of interest}

All authors declare that there are no conflicts of interest related to this manuscript.

\section{Ethical approval}

This manuscript does not report on any experiments for which ethical approval was required.

\section{Open Access}

This article is distributed under the terms of the Creative Commons Attribution 4.0 
International License (http://creativecommons.org/licenses/by/4.0/), which permits unrestricted use, distribution, and reproduction in any medium, provided you give appropriate credit to the original author(s) and the source, provide a link to the Creative Commons license, and indicate if changes were made.

\section{Electronic supplementary material}

The online version of this article (doi:10.1007/s11306-016-1057-y) contains supplementary material, which is available to authorized users. 


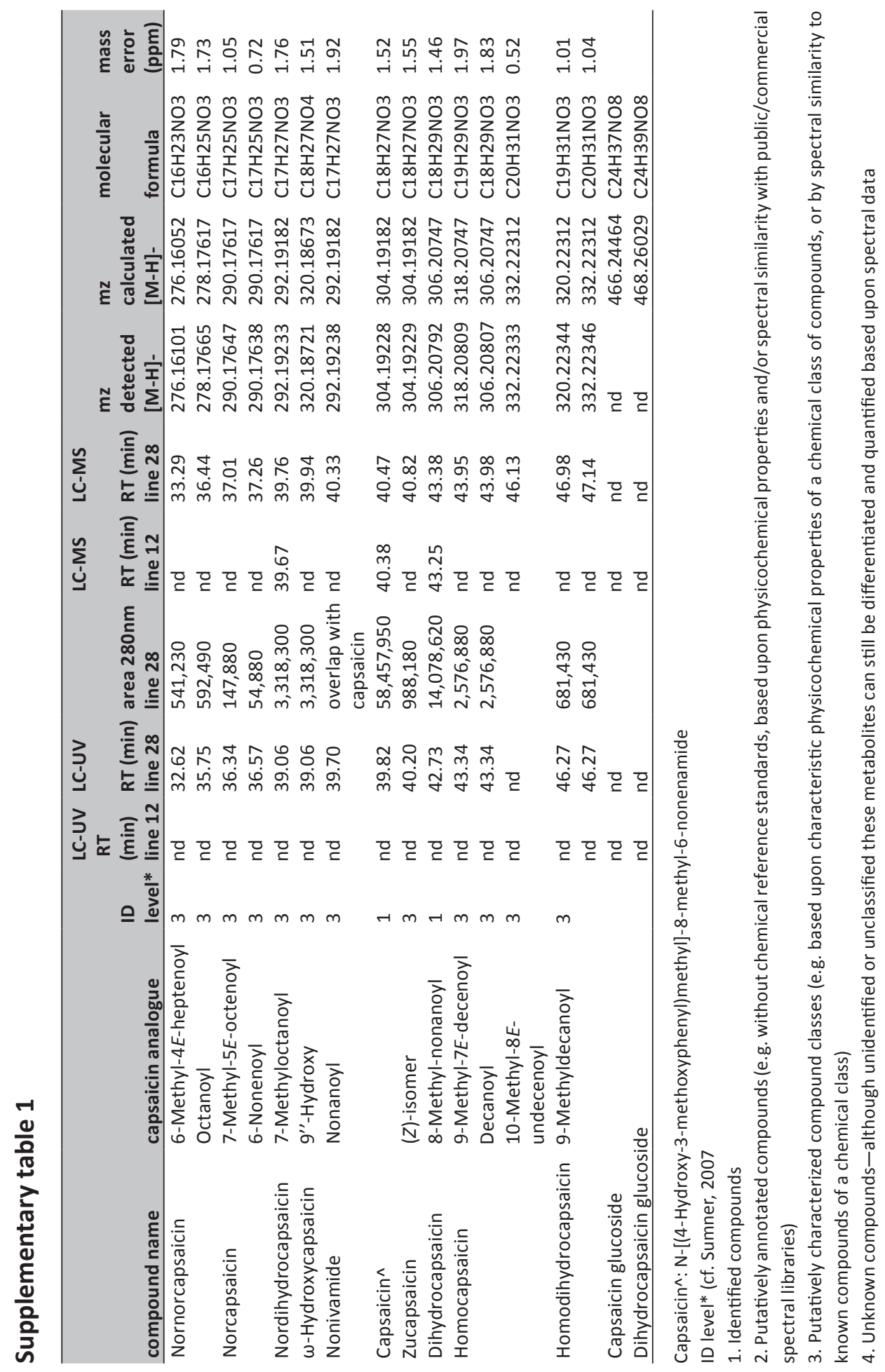



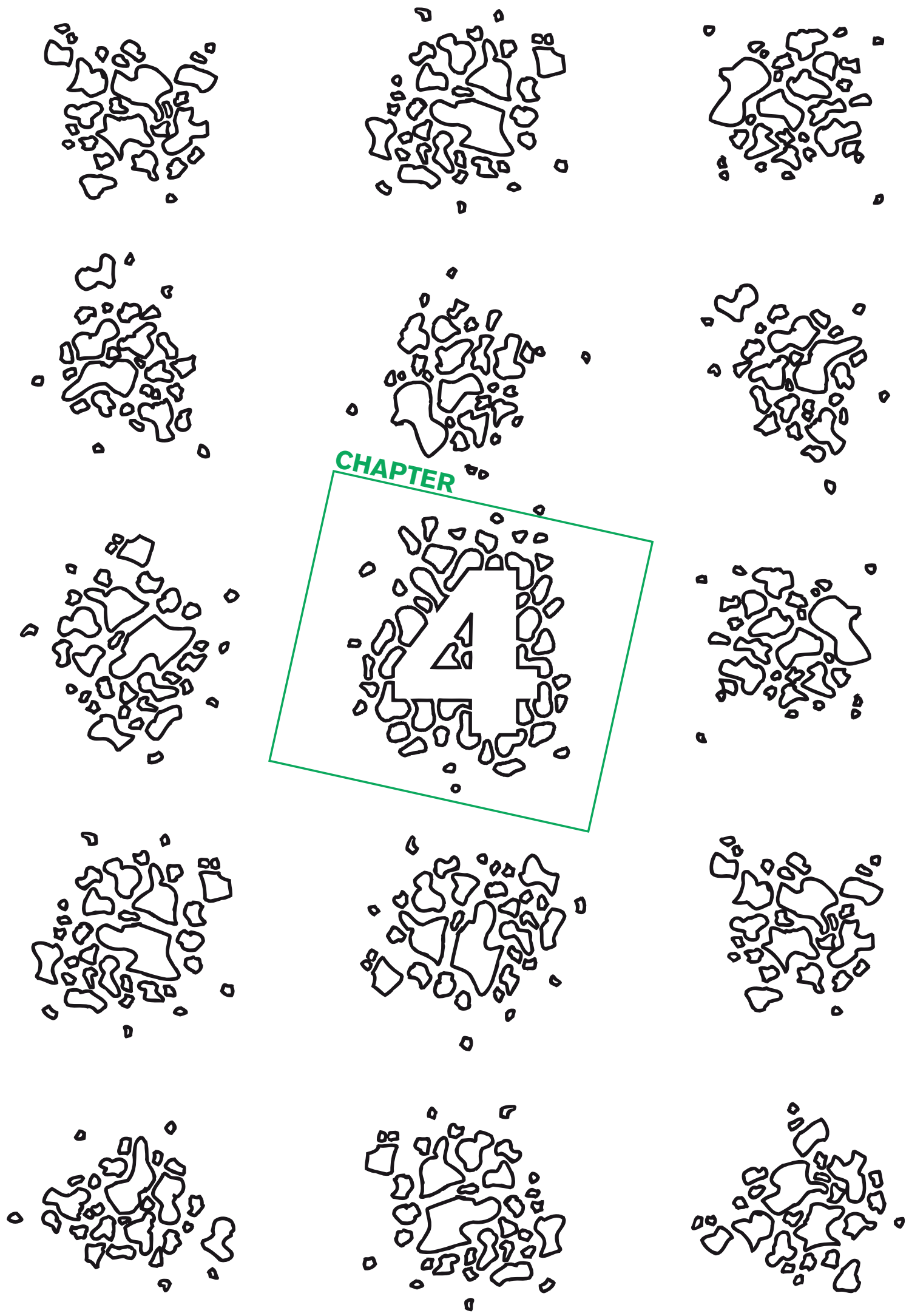


\section{Calcium imaging of GPCR activation using arrays of reverse transfected HEK293 cells in a microfluidic system}

Margriet Roelse, Maurice G.L. Henquet, Harrie A. Verhoeven, Norbert C.A. de Ruijter, Ron Wehrens, Marco S. van Lenthe, Renger F. Witkamp, Robert D. Hall, and Maarten A. Jongsma 


\begin{abstract}
Reverse-transfected cell arrays in microfluidic systems have great potential to perform large-scale parallel screening of G protein-coupled receptor (GPCR) activation. Here we report preparation of a novel platform using reverse transfection of HEK293 cells, imaging by stereo-fluorescence microscopy in a flowcell format, real-time monitoring of cytosolic calcium ion fluctuations using the fluorescent protein Cameleon, and analysis of GPCR responses to sequential sample exposures. To determine the relationship between DNA concentration and gene expression we analyzed cell arrays made with variable concentrations of plasmid DNA encoding fluorescent proteins and the Neurokinin 1 (NK1) receptor. We observed pronounced effects on gene expression of both the specific and total DNA concentration. Reverse transfected spots with NK1 plasmid DNA at $1 \%$ of total DNA still resulted in detectable NK1 activation when exposed to its ligand. By varying the GPCR DNA concentration in reverse transfection, the sensitivity and robustness of the receptor response for sequential sample exposures was optimized. An injection series is shown for an array containing NK1 receptor, bitter receptor TAS2R8 and controls. Both receptors were exposed 14 times to alternating samples of two ligands. Specific responses remained reproducible. This platform introduces new opportunities for high throughput screening of GPCR libraries.
\end{abstract}




\section{Introduction}

Reverse transfection is a technique to create arrays of transiently transfected cells on a solid surface. Since the first publication of Ziauddin and Sabatini in Nature in 2001 [78], the technique has found versatile application addressing different cellular and biological questions using printed cDNAs, siRNAs, viruses and even chemical compounds [164-169]. The generation of cell arrays through reverse transfection using plasmid DNA involves three basic steps: (i) printing a DNA micro-array, (ii) coating the DNA microarray with transfection reagents, and (iii) seeding with adherent cells to cover the pre-treated DNA micro-array. Steps (i) and (ii) are sometimes combined [166, 170, 171], but typically these three basic steps can transform a printed DNA microarray into a transfected cell array via efficient contact-mediated uptake of plasmid DNA by the adhering cells. This is visualized in Figure 1, steps 1 and 2, Video S1 and Figure S1. Protein expression efficiency depends, to a great extent, on correct formation and delivery of DNA transfection complexes. These aspects have been studied for both surface-immobilized and solution-mediated transfection systems [172-174]. Other important parameters influencing protein expression efficiency are the cell cycle $[175,176]$ the concentration [177-179], and the purity of plasmid DNA [180]. Higher plasmid concentrations have a direct effect on intracellular plasmid copy numbers in both the cytoplasm and the nucleus up to a certain maximum. Cohen et al [177] have shown that with increasing DNA levels, the maximum gene expression depends on cell type, the vector used and the method of transfection, so that no single protocol can be applied in all situations.

Only few studies have combined reverse transfection with microfluidics [84, 181-183]. In those studies, each spot of the cell array was enclosed by a chamber that allowed individual fluidic control. These micro-chambers mimic a multi-well plate where each well or chamber is individually used for measurements. However, a set-up applying controlled fluidics to individual spots is relatively complex for generic screening of GPCR libraries. This merited developing a system allowing simultaneous exposure of multiple receptors within a single chamber, thereby simplifying the set-up and increasing the efficiency of screening and analysis. In this article we show how we have developed such a screening system involving an array in a single flowcell and a stereomicroscope to simultaneously image all spots over time (Figure 1, steps 3 and 4, Video S1 and Figure S1).

Reverse-transfected cell arrays have been used before to screen GPCR libraries, but those arrays were small and spotted in the static environment of 96 well plates $[81,85]$. These setups did not allow for a sequential microfluidic screening of samples. The latter enables not only a dynamic analysis of receptor-ligand interaction, but also a more powerful spotbased, statistical analysis of sequential injections. In the present study, we placed a reverse transfected cell array in a re-sealable microfluidic chamber that covered the entire array (Figure S1). This set-up allowed routine monitoring of the ionic calcium responses of each individual spot to sequentially injected samples. As a proof-of-concept, we used the NK1 

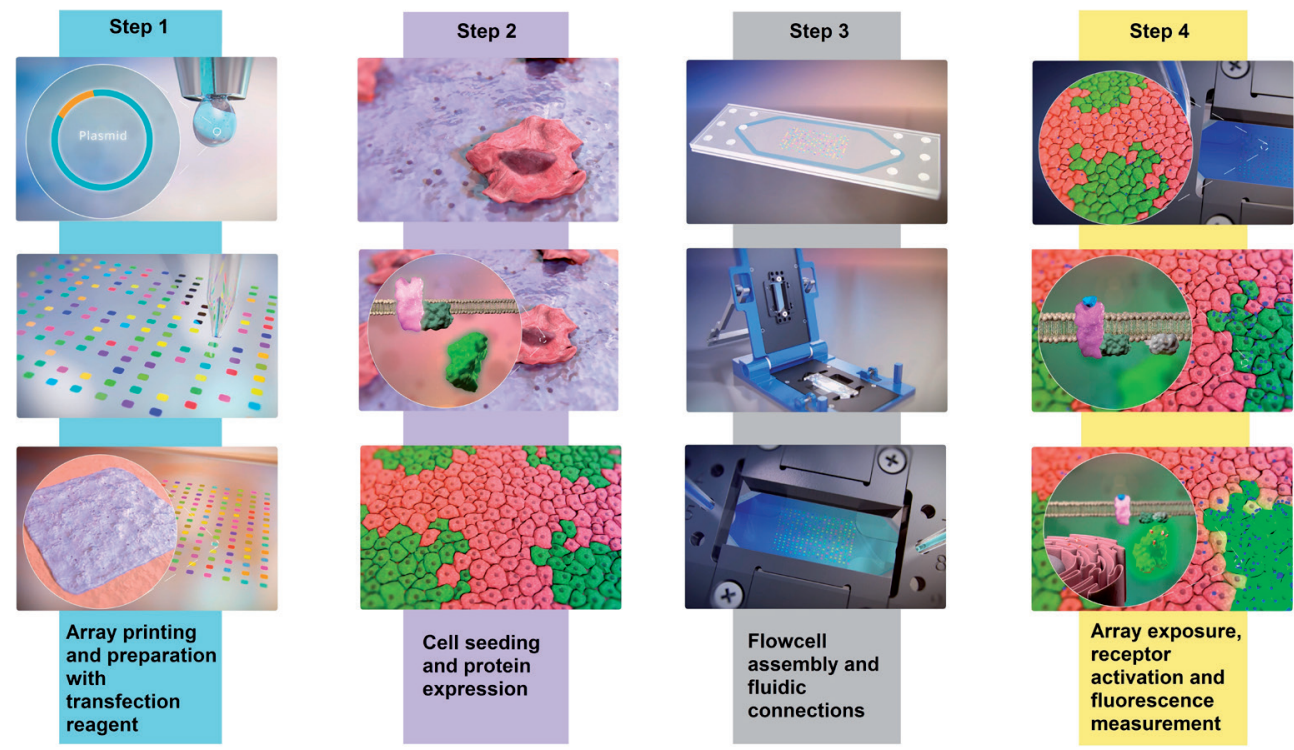

Figure 1, Schematic overview of array preparation and measurement in the microfluidic system. Steps 1 and 2 show array preparation involving DNA spot contact printing, overlay with transfection reagent, subsequent seeding of cells and expression of DNA after cell division. Step 3 shows the assembly of the flowcell and placement into the Micronit FluidConnect Pro flowcell holder. Once the holder is closed, fluidic connections are established and the microfluidic system produces a pressure controlled flow. Specific volumes of samples are injected sequentially into the constant flow to stimulate cell responses on the micro array (Step 4). Continuously, the entire array within the flowcell is imaged to capture the fluorescence from the calcium indicator YC3.6 over time and allow high-throughput screening. See also Supplementary Materials Video S1 and Figure S1.

receptor which was previously characterized in this flowcell $[95,96]$ and the bitter taste receptor hTAS2R8 [33]. The activation of these receptors by their respective ligands led to a transient increase in the cytoplasmic calcium ion concentration via a signal transduction pathway involving uncoupling of the $\mathrm{G}$ proteins, activation of phospholipase $\mathrm{C}$, inositol trisphosphate $\left(\mathrm{IP}_{3}\right)$ production, and opening of an $\mathrm{IP}_{3}$-gated calcium channel of the endoplasmic reticulum $[67,137]$. Such calcium responses are transient and were monitored real-time using the fluorescent calcium-ion sensor protein Cameleon YC3.6 [125, 131, 184] by means of FRET imaging. Previously, we expressed the NK1 and YC3.6 genes from a single plasmid. Here, we have designed and applied a more flexible system using separate plasmids for each gene. This enabled the modulation of receptor gene expression, independently of calcium sensor expression, by modifying plasmid DNA ratios. In this paper, we focused on the parameters of the reverse transfection procedure to optimize GPCR response in our microfluidic system and on the appropriate data analysis. In such a system, the following properties are paramount for optimal use: protein expression efficiency; transfection uniformity over the array; co-transfection and a reproducible response to repeated challenges (robustness). The potential of this system for high throughput screening of GPCR receptor libraries is discussed. 


\section{Materials and Methods}

An animation of the array preparation, flowcell assembly and array measurement is presented in Supplementary Materials Video S1 and Figure 1

\section{Expression vectors}

The NK1 receptor gene was purchased at the UMR cDNA Resource Center (University of Missouri-Rolla, cat.nr. TACR100000). The hTAS2R8 (NCBI Reference Sequence: NM_023918.1) bitter receptor gene was PCR amplified from genomic DNA from HEK293 cells and cloned into a pcDNA3 expression vector. This vector contained a DNA sequence encoding the first 45 amino acids of the rat somatostatin receptor type 3 to improve membrane targeting of the bitter receptor protein. The $G \alpha_{16 G U S T 4}$ gene in vector pcDNA3 was a gift from Dr Takashi Ueda (Nagoya City University, Nagoya, Japan). The YC3.6 gene in pCDNA3 (ThermoFisher scientific, Waltham, MA) was a gift from Prof. Roger Tsien (UC San Diego). Plasmid containing CFP and RFP were from Addgene (\#13030 and \#13032 respectively). All plasmids used were of similar size and carried the same CMV promotor.

\section{Cleaning and printing of DNA arrays}

Glass slides (15x45 mm) were cleaned with 2\% Hellmanex solution (Hellma Analytics, Müllheim, Germany) by dipping the slides in the solution, sonication for 15 minutes and subsequent incubation for a maximum of 2 hours at room temperature. The slides were then thoroughly rinsed and incubated overnight at room temperature in ultra-pure water to remove any residue left by the Hellmanex solution. After incubation, the slides were dried in a $50^{\circ} \mathrm{C}$ incubator and coated for one hour with poly-L-lysine $100 \mu \mathrm{g} / \mathrm{ml}$ solution (Sigma, St. Louis, MO, P1274). The slides were then rinsed with ultra-pure water, dried and stored at room temperature in a dust free container until use. The slides were printed by a Microgrid II DNA printer (BioRobotics) using modified SMP3 pins (arrayit) which were slightly blunted to generate square $\sim 200 \mu \mathrm{m} \times 200 \mu \mathrm{m}$ spots (Figure S2B). Slides were printed at $\sim 55 \%$ relative humidity and left to dry for one hour after printing. The spots were initially printed using a $400 \mu \mathrm{m}$ grid spacing, but as the resulting transfected cell spots appeared at risk of cross-contamination because of slight cellular motility, spot spacing was subsequently increased to $600 \mu \mathrm{m}$ (Figure S2D). The print solution was composed of purified plasmid DNA (Qiagen, Plasmid Mini kit, 12123), 0.4\% gelatine (Sigma, G9391) and $50 \mathrm{mM}$ sucrose dissolved in ultra-pure water. The gelatine was prepared fresh before use by dissolving $1 \%$ gelatine in ultra-pure water at $60^{\circ} \mathrm{C}$ for 15 minutes and filtering it through a $0.2 \mu \mathrm{m}$ filter. After printing, the slides were stored at room temperature in a dust-free and dry container. Slides could be stored for up to 6 months without loss of transfection yields. Print solutions were freshly made, but can be stored at $-20^{\circ} \mathrm{C}$ and re-used after adjusting volumes and heating to $60^{\circ} \mathrm{C}$ for 15 minutes. 


\section{Cell culture and reverse transfection}

HEK293H cells (ThermoFisher scientific, Waltham, MA11631-017) were maintained as a monolayer in DMEM (Gibco, 21063-029) containing 1x MEM non-essential Amino Acids (Gibco, 11140-035), 1x Penicillin-Streptomycin (Gibco, 15140-122) and 10\% FBS (Gibco, 26140 ) in a $37{ }^{\circ} \mathrm{C}$ incubator with $5 \% \mathrm{CO}_{2}$. HEK293H cells stably expressing the chimeric $\mathrm{G}$ protein subunit $\mathrm{G} \alpha_{16 \mathrm{GUST} 44}$ [75] were used for the expression of hTAS2R8. The reverse transfection method was adapted from the Sabatini lab description [78]. In short, we prepared a transfection mix from the Effectene kit with transfection reagents (Qiagen, 301425) by mixing $75 \mu \mathrm{l}$ EC buffer with $8 \mu$ l enhancer, pre-incubating for 5 minutes at room temperature and then adding $12.5 \mu \mathrm{l}$ Effectene lipids. The transfection mix was vortexed for 10 seconds and carefully applied, dropwise, onto the glass surface submerging the entire $15 \times 18$ spot array. The slides were incubated for 20 minutes at room temperature. The transfection mix was removed by tilting the array and carefully pipetting off the transfection mix. Once the slides were air-dried, a flexiPERM chamber (Greiner, 90032039) that was cut to the appropriate size, was mounted over the array (Figure S1A). The $0.9 \mathrm{~cm}^{2}$ chamber was filled from the edge with HEK293H cells $\left(2.2 \times 10^{5}\right.$ cells $\left./ \mathrm{cm}^{2}\right)$ and incubated at $37^{\circ} \mathrm{C}$ incubator with $5 \% \mathrm{CO}_{2}$ for at least 27 hours before use.

\section{Flowcell and array imaging setup}

The reverse transfected arrays ( 270 spots and $\sim 100 \mathrm{~mm}^{2}$ ) were mounted with a resealable borosilicate glass flowcell (Micronit Microfluidics B.V, Resealable FC 4515, Extended 10 $\mathrm{mm}$ ) and fitted in a flowcell holder (Micronit Microfluidics B.V., Fluidic Connect PRO Chip Holder with 4515 Inserts, Figure S1A). The arrays were imaged by a Leica fluorescent stereo microscope (Leica M205FA with DFC 345 FX camera and 2.0x PlanApo objective with NA 0.35) fitted with a 0.32x C-Mount and filters for CFP (ET CFP 10447409, exc. 436/20 and em. 480/40) and YFP FRET (ET FRET 10450566, exc. 436/20 and em. 535/30). Lamp intensity (Osram, HXP-R 120W/45C VIS) was set at maximum and exposure time was 400 msec. Large cell arrays as shown in Figure S1B and S3 were imaged at maximal field of view (FOV) in order to image fluorescent signal from the entire array. Compared to the confocal microscope images of Figure $2 \mathrm{~B}\left(\sim 0.16 \mathrm{~mm}^{2}\right)$, this led to a loss of cellular resolution due to the larger working distance, but a $\sim 625 x$ larger area that was imaged. From Figure S3B it is evident that due to this zooming out, cellular contours were lost and that a single pixel of about $11 \mu \mathrm{m}$ diameter, can cover one or partially two cells. In the time series acquisition a $2 x$ binning was used resulting in a $23 \times 23 \mu \mathrm{m}$ pixel size on chip, which corresponds with single HEK cells in the sample. The variation in fluorescent protein expression between cells is therefore correlated with the variation in individual pixel intensity. The graph in Figure S3C shows the pixel intensity against the ranked pixels of the enlarged section. At 8-bit resolution, pixels with a fluorescence intensity of 256 have reached the detector limit. Pixels that represent the background in the selection (e.g. at the edge of this section) have a background intensity of 0- 20, and pixels with intensities between $\sim 20$ and 256 
represent those with fluorescent protein expression. To distinguish fluorescent pixels from the background we set a lower limit (threshold) using the background algorithm in the CellProfiler software. On large arrays expressing the receptor proteins, calcium responses from receptor activation were imaged in time. A continuous flow of $100 \mu \mathrm{l} / \mathrm{min}$ culture medium or microscopy buffer ( $\mathrm{NaCl} 130 \mathrm{mM}, \mathrm{KCl} 5 \mathrm{mM}$, Glucose $10 \mathrm{mM}, \mathrm{CaCl}_{2} 2 \mathrm{mM}, \mathrm{HEPES}$ $10 \mathrm{mM}$ at $\mathrm{pH}$ 7.4) was applied for 10 minutes before the samples were injected. Sample injections were done using a manual injection valve (Upchurch, V-451) containing a 50 $\mathrm{\mu l}$ loop. Channel gains were maintained after initial separate adjustment for CFP and YFP FRET channels to measure fluorescence intensity amplitudes and minimize pixel saturation of the reverse transfected array. Images were taken every 3-5 sec (Figure S1B).

\section{Live imaging of reverse transfection time course}

A series of images of the reverse transfected cell array was taken to monitor cell motility and fluorescence intensity in time. Minimal light stress during acquisitions was achieved by spinning disk confocal imaging (Andor-Revolution system with Yokogawa disk) on a Nikon Ti Eclipse inverted microscope equipped with autofocus (PFS3), piezo stage (ASI XY-LE) and EMCCD camera (Andor Ixon888, 1024x1024 pixels, 13×13 $\mu \mathrm{m}$ ). Optimal physiological conditions were maintained by a stage top incubator (Tokai Hit, Shizuoka, Japan) at $37^{\circ} \mathrm{C} \pm$ $0.1^{\circ} \mathrm{C}$ with continuous humidified $5 \% \mathrm{CO}_{2}$ flow. Images were acquired every 30 minutes over a period of 33 hours with a 10x CFI Plan-Fluor NA 0.3). Signal expression from CFP, YFP or RFP was monitored in corresponding domains of the reverse transfected cell array. Images were recorded with MetaMorph Imaging series 7.8.3 (Molecular Devices).

\section{Data analysis of fluorescent protein expression}

Pixel intensity data were obtained from the Leica software (LAS AF Lite, version 3.1.0). A grid of equally sized regions of interest (ROI) was created, each containing one array spot. The intensity histogram of each ROI was then exported to a spreadsheet program. There, the pixels were sorted against the intensity value and numbered, starting with the highest intensity value. When ranked pixels from different reverse transfection conditions (e.g. input DNA concentration) were compared, a ranking range was chosen that yielded a value between the upper and lower thresholds for each condition. To avoid skewed data comparisons, only pixels in this dynamic range rather than an $\mathrm{ROI}$ average were used to yield a valid analysis of DNA concentration effects.

\section{Data analysis of calcium responses}

The images recorded on the Leica fluorescence stereo microscope were first analyzed using CellProfiler software version 2.1.1 [185]. Two CellProfiler pipelines were created. In the first pipeline, one YFP image was used to create a mask of the array. This mask separates signal pixels from background pixels and is based on the threshold algorithm given in the pipeline. In the second pipeline, a grid overlay was created to define the array spot positions. The 
mask image from the first pipeline was used as overlay on every CFP and YFP image of the recorded time series. The intensity values of the pixels within the mask for both CFP and YFP images were measured and the signal was defined by calculating YFP/CFP ratios. Peak heights were then determined for all spots and all injections by measuring the range of the signal within the first 40 cycles. In order to be able to compare receptor responses to different types of injection a linear mixed model was constructed relating the injection type to the receptor type. Since different spots of the same receptor type can sometimes have quite different baseline responses, the spot number was taken into account as a random factor. The outcome of the model consists of estimated responses (including confidence intervals) of each receptor type to all injection types. The estimated differences with a reference injection (here the blank) can then be visualized, including confidence intervals calculated for each injection type separately. Model calculations were performed using in house software (publication in preparation)

\section{Results}

Time course of protein expression after reverse transfection at the cell and spot level Protein expression of fluorescent proteins was followed live and used to establish the timecourse of protein accumulation both at the single cell and the reverse-transfected spot levels. Fluorescence emission of a reverse transfected spot carrying the RFP plasmid DNA was imaged using a spinning disk confocal fluorescence microscope at 30 minute intervals for a period of $33 \mathrm{~h}$. (Figure 2, Video S2). Imaging was initiated directly after seeding the cells on top of a DNA array pre-treated with Effectene. Cells became fully attached to the surface in ca. $5 \mathrm{~h}$, initially covering about $50 \%$ of the slide. At 3-5 h post cell inoculation, the first cells started to show detectable fluorescence above background levels. To follow this in detail, we counted the number of transfected cells at each time point and measured the total fluorescence intensity of the image (Figure 2A). It should be noted that accurate counting of fluorescent cells was difficult, because these tended to overlap and move between images which is a sign of vitality in HEK293 cells [186]. Together with effects of cell division this did result in fluctuations of the number and intensity of (ranked) cells (Figure $2 \mathrm{~A}, \mathrm{C}$ ). The total fluorescence of the image showed a nearly linear increase between 14 - 30 $h$, post inoculation (Figure $2 \mathrm{~A}$ ). We also observed a large variation in fluorescence intensity between the transfected cells as shown in Figure 2B, even though the spot was transfected with a fixed RFP-encoding plasmid DNA concentration. The range in fluorescence intensity between individual cells increased over time. To understand this, we ranked the fluorescence intensities from high to low for each time point, and plotted the average intensities in pools of 10 cells per rank, also from high to low, as shown in Figure 2C. We observed that the fluorescence of cells did not decrease in time or increase faster when detected later. Thus, the figure shows that cell groups with early expression of RFP indeed reach the highest 

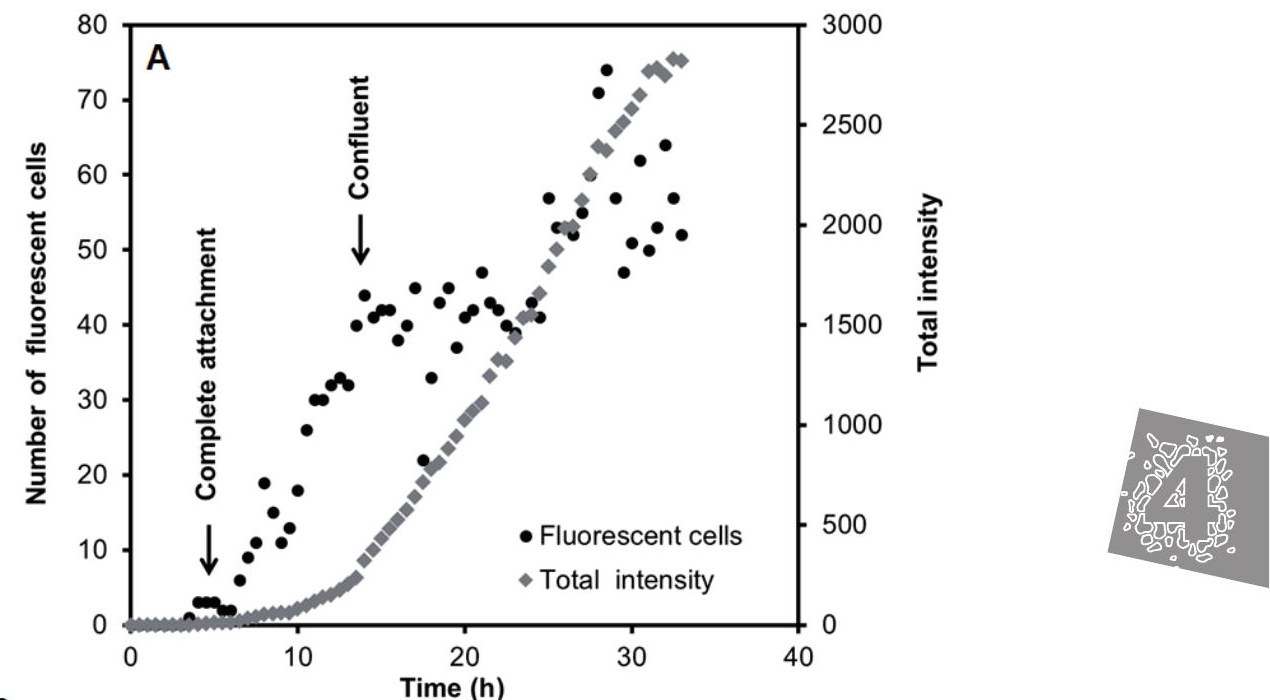

B
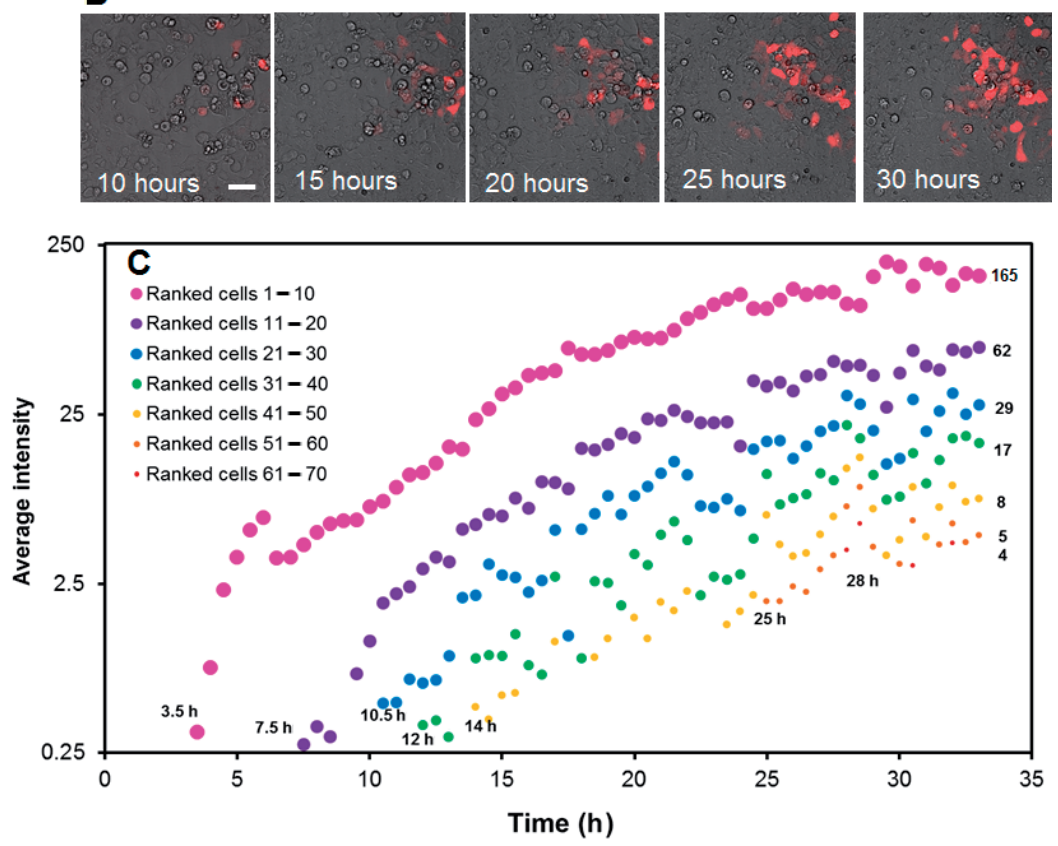

Figure 2, Time course of RFP expression after reverse transfection. A, Graph of the number of fluorescent cells (left axis, black dots) and the total fluorescence of all cells (right axis, open diamonds) in time. Based on bright field images, the complete cell attachment and the moment when complete confluence of the cell layer was reached are indicated in the figure. B, Overlay of bright field and RFP fluorescence at 10, 15, 20, 25, and 30 hours after inoculation. Scale bar $=50 \mu \mathrm{m}$. C, Graph of the average intensity within pools of ranked fluorescent cells over the time course of the reverse transfection. The pools represent up to 10 fluorescent cells ranked from the highest to lowest expressing cells at each timeframe. The start time of each pool is indicated in the graph, and the average intensity of the pool at $33 \mathrm{hrs}$ post transfection is shown on the right within the graph. 
final level. The delay in visible RFP expression in subsequent classes of cells is not only characterized by a time delay but also by lowered maxima. For example, a 4 hour delay in expression of the first two classes, resulted in a circa 2.5 fold lower RFP expression level after 30 hours, and a 10 hour delay in a 30 fold lower expression. Since the variation in RFP expression within the reverse transfected spot was large, we investigated, given the intercellular variation in the expression of proteins, how large reverse transfected DNA arrays could best be imaged by fluorescence stereomicroscopy and how expression relates to the DNA dosage.

\section{Effect of DNA concentration on protein expression}

To determine the effect of DNA concentration on protein expression, an array was prepared using various concentrations of CFP plasmid DNA. A fluorescence image of a representative part of this array is shown in Figure 3A, with over-exposed pixels in blue. Like Figure 1, each spot in Figure 3 contains pixels with a wide range of fluorescence intensity levels which reflects the differences in cellular expression levels. In these recordings the detector gain was deliberately set to allow some saturation at the highest expressing cells in order to keep the low expressing cells within the detection range. With increasing DNA concentration an increase in both fluorescence intensity and number of pixels per spot was observed (Figure $3 B$, Figure S2), which also increased the number of saturated pixels. If saturated pixels would be included in an analysis of the effect of different total DNA concentrations, expression efficiency would be underestimated. Vice versa, leaving out underexposed pixels with no

Figure 3, The effect of total DNA concentration and coding DNA concentration on protein expression * displayed as pixel intensity. The boxplots of Figure 3 show the distribution of spot intensities per category. Each category includes about 10 spots. The black line indicates the median value of the data distribution, the box represents the first and third quarter of the data distribution and the whiskers represent the minimum and maximum value of the data set. The open circles are outliers in the data set. A, Fluorescence image of 4 spot types representing from left to right 10, 20, 30 and 40 $\mathrm{ng} / \mu \mathrm{l}$ total DNA. The image is shown with a range indicating LUT where over-exposed pixels display in blue. Scale bar $=200 \mu \mathrm{m}$. B, Average spot intensity (based on pixels above background intensity including saturated pixels) of spots containing total DNA at concentration of $10 \mathrm{ng} / \mu \mathrm{l}$ ( $\mathrm{n}=9$ spots), $20 \mathrm{ng} / \mu \mathrm{l}$ ( $\mathrm{n}=8$ spots), $30 \mathrm{ng} / \mu \mathrm{l}$ ( $\mathrm{n}=9$ spots) and $40 \mathrm{ng} / \mu \mathrm{l}$ ( $\mathrm{n}=8$ spots). Numbers above the boxplots indicate the average amount of pixels of the spots. C, Pixel ranking of spots containing $0 \mathrm{ng} / \mu \mathrm{l}, 10$ $\mathrm{ng} / \mu \mathrm{l}, 20 \mathrm{ng} / \mu \mathrm{l}, 30 \mathrm{ng} / \mu \mathrm{l}$ and $40 \mathrm{ng} / \mu \mathrm{l}$ total DNA. The grey dashed lines show an upper limit (with value 256) and a lower threshold (background level with value 20 ). The grey area (pixel 110-212) is the range of pixels where all spots can be compared using the same pixel range and within the upperand lower limits. D, Boxplots of CFP fluorescence intensity of all pixels in range (grey bar) as indicated in C with increasing total CFP plasmid DNA concentration. E, Boxplots of CFP fluorescence intensity of all pixels in range with $10 \mathrm{ng} / \mu \mathrm{l}$ ( $\mathrm{n}=9$ spots), $20 \mathrm{ng} / \mu \mathrm{l}(\mathrm{n}=9$ spots) and $30 \mathrm{ng} / \mu \mathrm{l}$ ( $\mathrm{n}=9$ spots) total DNA and constant $10 \mathrm{ng} / \mu \mathrm{l} \mathrm{CFP}$ plasmid DNA. F, Boxplots of CFP fluorescence intensity of all pixels in range with increasing CFP plasmid DNA concentration and constant total DNA concentration. The array of Figure $3 F$ was measured on a different array (all conditions $n=26$ spots) with a 12 bit intensity scale (4096 levels) leading to higher intensity values and a different pixel range for intensity comparison than $\mathbf{D}$ and $\mathbf{E}$. 
value leads to overestimation of expression efficiency. This is shown graphically in Figure $3 \mathrm{C}$ using a pixel ranking of all pixels in spots transfected using 0-40 ng/ $\mu$ l CFP plasmid DNA. In this plot the lower threshold separates signal from background so that spots generated with $0 \mathrm{ng} / \mu \mathrm{l}$ DNA show no fluorescence. Fluorescence of CFP spots was measured up to an intensity value of 256 , the limit of the detector. The grey bar in Figure $3 C$ shows a range of comparable, ranked pixels with non-saturated fluorescence above the lower threshold at all four concentrations tested. For these pixels, intensity reports the direct relationship between protein expression and DNA concentration.

In Figure 3D boxplots of all pixel intensities within the pixel range of Figure $3 \mathrm{C}$ are shown. A two-fold increase in CFP fluorescence is observed from 10 and $20 \mathrm{ng} / \mu \mathrm{l}$, but between
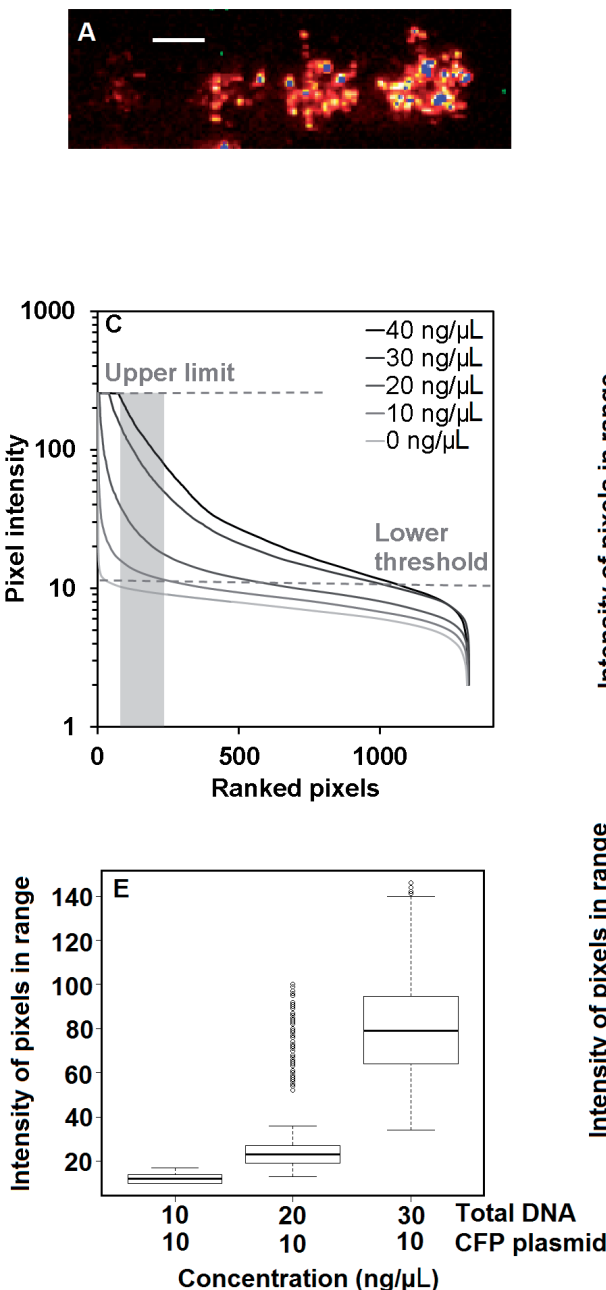

Concentration $(\mathrm{ng} / \mathrm{\mu L})$
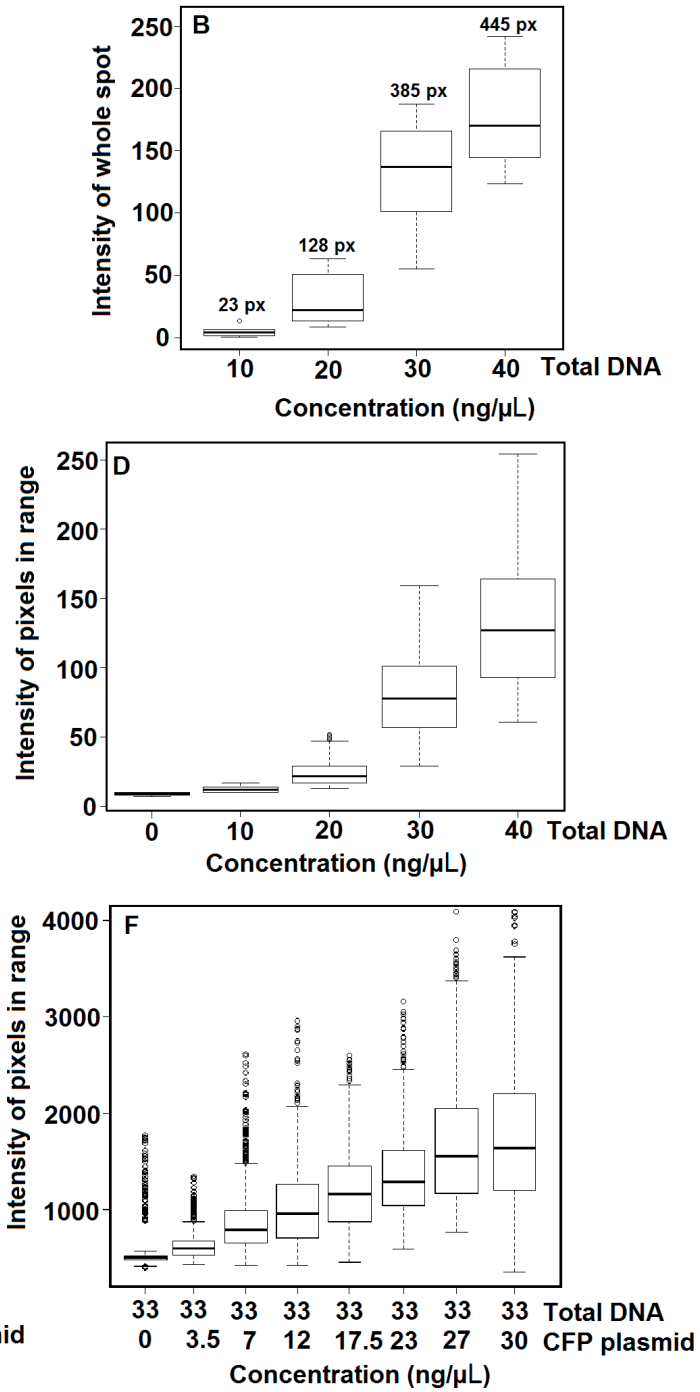
10 and $40 \mathrm{ng} / \mu \mathrm{l}$ there is almost a ten-fold increase in CFP expression, suggesting that the relationship between protein expression and total DNA concentration is not linear. Since standard deviations increase with increasing DNA concentration, a linear relationship with the log-transformed CFP intensities can be expected. This indeed leads to a high R2 value of 0.89 (compared to 0.68 for the untransformed CFP intensities).

\section{Effect of co-transfection on protein expression}

To investigate non-linear effects of DNA concentration on protein expression, two arrays were prepared keeping either the CFP plasmid DNA concentration or the total DNA concentration constant. With a constant CFP plasmid DNA concentration, the dependency of cell transfection on total DNA would be established and with a constant total DNA concentration, the dependency of CFP expression on DNA plasmid copy number would become visible. Figure 3E shows the intensity of CFP fluorescence with increasing total DNA concentration, but at a constant CFP plasmid DNA concentration of $10 \mathrm{ng} / \mu \mathrm{l}$. This figure follows the same pixel intensity increase as in Figure 3D. This indicates that, in this concentration range, CFP expression almost entirely depended on the total DNA concentration rather than the specific CFP plasmid DNA concentration. Figure 3F shows the intensity of CFP fluorescence with $33 \mathrm{ng} / \mu \mathrm{l}$ total DNA concentration and an increasing proportion of CFP plasmid DNA. Despite the large variation, there was a linear relationship between the concentration and the average CFP intensity, described by the following formula:

pixel intensity $=39.6[$ CFP $]+534$

This indicates a background fluorescence of 534 and a slope coefficient of 39.6 for the increase of CFP fluorescence in this array, with an $R^{2}$ value of 0.985 . Also at the highest DNA concentration no clear saturation effects were visible.

\section{Effect of DNA concentration on NK1 receptor calcium signaling}

Considering the observed effects of DNA concentration on protein expression, we next studied the effects of the coding DNA concentration on the functional activation of the G-protein coupled receptor neurokinin 1 (NK1). The transient cytoplasmic calcium signal was measured with the co-transfected calcium sensor protein Cameleon YC3.6. All spots were transfected with the same amount of calcium sensor resulting in similar total fluorescence levels between spots. The optimum gain was set to maximize the number of fluorescent pixels. This led to some overexposed pixels which were omitted from the analysis.

The first question we addressed was the relationship between receptor DNA concentration (ranging from $0.3 \mathrm{ng} / \mu \mathrm{l}$ to $15.8 \mathrm{ng} / \mu \mathrm{l}$ in print solutions of $33 \mathrm{ng} / \mu \mathrm{l}$ total DNA) and the receptor activation response. Transfected arrays were assembled in a microfluidic flowcell 

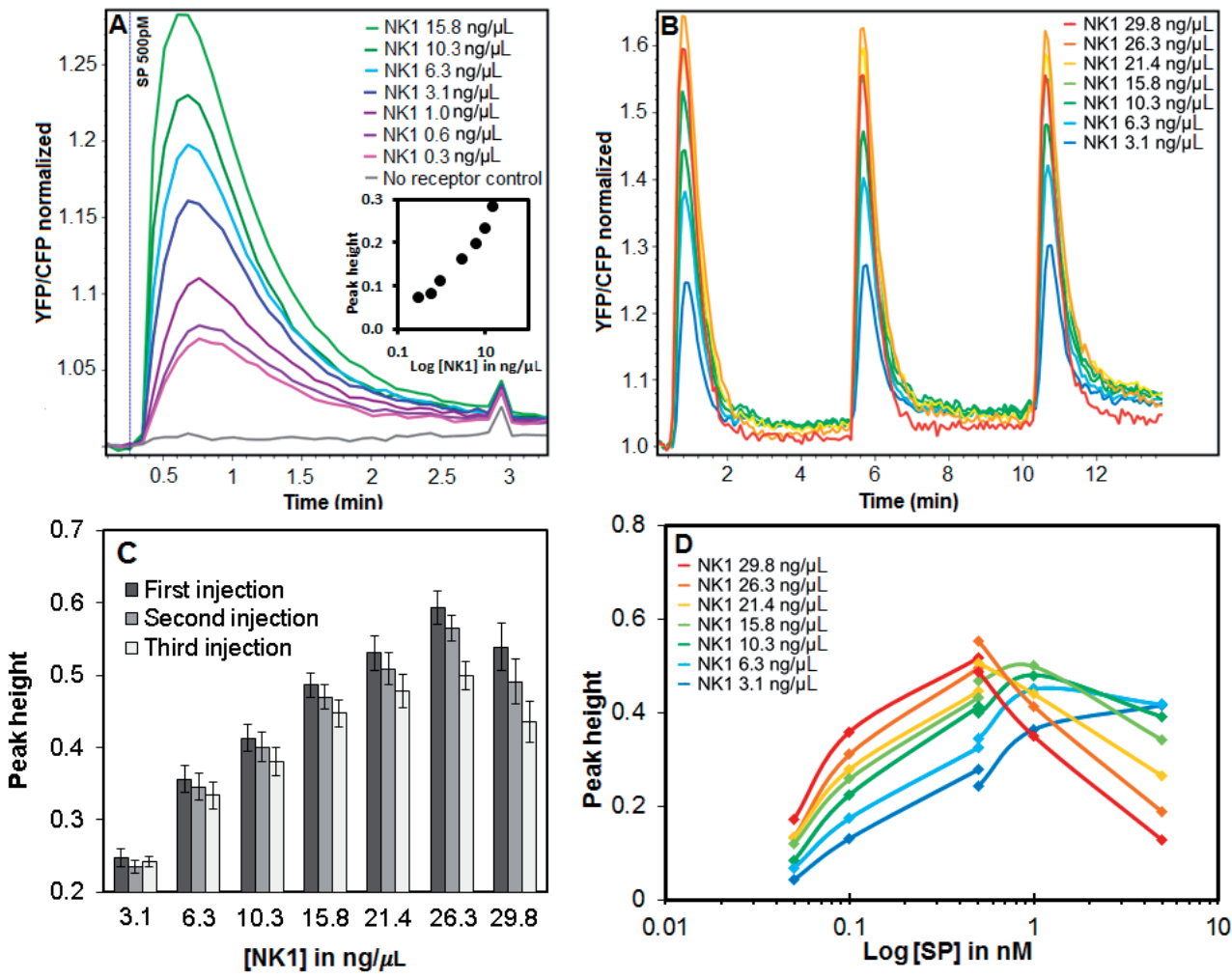

Figure 4, Calcium ion dynamics after SP exposure to arrays co-transfected with NK1 receptor and YC3.6 calcium sensor. Calcium ion dynamics are displayed in YFP/CFP signal ratio obtained from the YC3.6 calcium sensor and normalized by dividing the CFP and YFP signals by the average of the first images before sample exposure. A, Cytosolic calcium ion transients after 500 pM SP exposure rise with increasing NK1 plasmid DNA concentration ranging from $0.3 \mathrm{ng} / \mu \mathrm{l}$ to $15.8 \mathrm{ng} / \mu \mathrm{l}$ while total DNA concentration was kept at $33.3 \mathrm{ng} / \mu \mathrm{l}$. Inset chart shows a correlation between NK1 plasmid DNA concentration and peak height. B, Repeated injections of 500pM SP on an array with total DNA concentration of $33.3 \mathrm{ng} / \mu \mathrm{l}$ and NK1 plasmid DNA concentration ranging from $3.1 \mathrm{ng} / \mu \mathrm{l}$ to $29.8 \mathrm{ng} /$ $\mu \mathrm{l}$. C, Bar chart of B showing the peak height and standard error for each injection of 500pM SP at the different NK1 plasmid DNA concentration. D, Dose response curve for NK1 receptor and SP ranging from $50 \mathrm{pM}$ to $5 \mathrm{nM}$ SP. The curve was measured over two arrays as shown by the interruption in the curves. All values in this figure represent an average of over 20 spots each, distributed equally over an array.

with a continuous flow of $100 \mu \mathrm{l} /$ min culture medium. Samples of Substance P (SP), an 11 amino-acid neuropeptide which activates the NK1 receptor, were injected using a $50 \mu \mathrm{l}$ sample loop, resulting in a transient exposure to SP of about 30 seconds. Fluorescence emission of the calcium sensor protein was ratio-imaged every $5 \mathrm{sec}$. After 2 minutes the cytosolic calcium ion level had returned to basal levels, indicating a rapid and full recovery. SP was re-injected every 5 minutes. Figure $4 \mathrm{~A}$ shows the changes in fluorescence ratio of the YC3.6 sensor after injections of 500 pM SP. The inset of Figure 4A shows a linear 
correlation of the calcium signal maxima to the log of the concentrations between 0.6 and $10.3 \mathrm{ng} / \mu \mathrm{l}$ NK1 plasmid DNA. Spots with the lowest NK1 plasmid DNA concentration of $1 \%$ of total DNA yielded a calcium response which was only $\sim$-fold less than the response of spots with a 50-fold higher NK1 plasmid DNA concentration. In a second array (Figure $4 \mathrm{~B}$ ) we used an NK1 plasmid DNA concentration of 3-30 $\mathrm{ng} / \mu \mathrm{l}$ relative to $33 \mathrm{ng} / \mu \mathrm{l}$ total DNA. This array was exposed to three sequential injections of 500 pM SP. While there was response variation ( 3 to $10 \%$ in peak height) between spots, at the spot level, responses were highly reproducible and quickly returned to baseline after each stimulation. Yet, at all NK1 plasmid DNA concentrations except the spots with $\sim 3 \mathrm{ng} / \mu \mathrm{l}$ NK1 plasmid DNA, a slight decrease in peak height was observed for subsequent injections at that high SP concentration. The largest decrease in signal, about $20 \%$ of peak height, was found in the spots where the highest NK1 plasmid DNA concentration has been used (Figure 4C). Not surprisingly, two-way ANOVA showed that both NK1 concentration and injection number were highly significant $(p<1 e-4)$.

Using this array we made dose response curves by sequentially injecting $3 \times 50$ pM, 3x 100 pM and 2x 500 pM SP onto one array and 3x 500 pM, 3x 1 nM and 1x 5 nM SP onto a second one. The averages of peak heights are shown in Figure 4D. We observed increasing peak heights for spots with increasing NK1 plasmid DNA concentrations up to $500 \mathrm{pM} \mathrm{SP}$. Thereafter, the trend reversed for spots with $15.8 \mathrm{ng} / \mu \mathrm{l}$ or more NK1 plasmid DNA. After the 500 pM SP injection, the subsequent injections of 1 and $5 \mathrm{nM}$ SP resulted in increasingly lower peak heights for those spots with high NK1 plasmid DNA concentrations. In contrast, a single injection of $5 \mathrm{nM}$ SP on a freshly prepared array resulted in the highest peaks from spots with the highest NK1 plasmid DNA (data not shown). The lowered maxima for the final $5 \mathrm{nM}$ injection in spots with $15.8 \mathrm{ng} / \mu \mathrm{l}$ or more NK1 plasmid DNA were a result of the injection sequence of this experiment.

\section{Specific receptor responses to repeated challenges}

In the previous section we described that within a certain receptor DNA concentration and amount of ligand, repeated injections produce similar receptor responses. Receptor responses are reproducible with repeated injections. In this section we show how receptors respond to a series of repeated sample injections in a large array containing $>60$ replicates of three types of transfected spots; one carrying the NK1 receptor, one the bitter R8 receptor, and one the control with mock DNA, all printed at $25 \mathrm{ng} / \mu \mathrm{l}$ for high sensitivity and all with $25 \mathrm{ng} / \mu \mathrm{l}$ YC3.6 coding DNA of the calcium sensor. Reverse transfection was performed with HEK293 cells that stably expressed the chimeric Galpha ${ }_{16 G U S T 44}$ G-protein to allow calcium signaling from bitter receptors [75]. The ready array was mounted into the flowcell and dose response curves were determined for each receptor for activation by chloramphenicol and SP (Figure 5A). At a selected non-saturating concentration ligands for both receptors were then intermittently injected every 3 minutes during a period of $>50$ min (Figure 5B). We observed specific and reproducible response patterns for each 

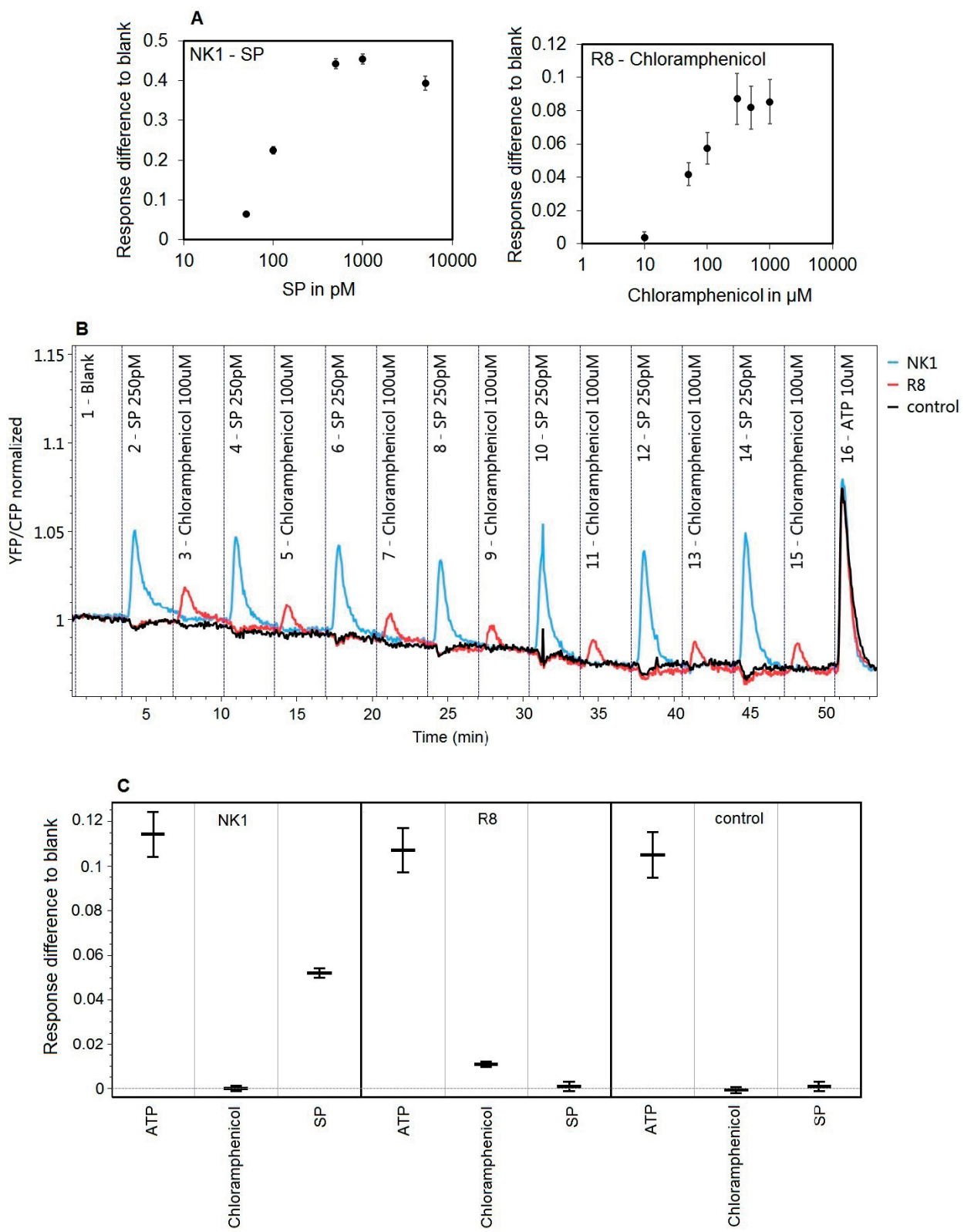

Figure 5, Repeated challenges of two distinct GPCR's by a series of samples. A, Dose response curves of both NK1 and TAS2R8 for SP and chloramphenicol on a different array. B, Average response profile of NK1, TAS2R8 and the no receptor control of the whole injection series. The first injection is a blank injection of assay buffer, injections 2 to 15 are sample exposures of 30 seconds to either $\mathrm{SP}$ at $250 \mathrm{pM}$ or Chloramphenicol at $100 \mu \mathrm{M}$ and the last injection 16 is ATP at $10 \mu \mathrm{M}$. Response profiles are an average of about 60 spots per spot type. For easier visual interpretation, responses were normalized by dividing the CFP and YFP signals by the average of the first images before sample exposure. C, Estimates and confidence intervals for receptor-specific responses (peak heights) to injections, compared to the blank injection. 
receptor / ligand combination relative to the control. Figure 5C summarizes receptorspecific responses to specific injections, expressed as differences with the response of the blank injection. NK1 gives a significant response to SP but not to Chloramphenicol; R8 shows the reverse behavior. All spots including the control respond to the last injection of ATP because it triggers the P2Y receptor which is natively expressed by the HEK293 host cells [187].

\section{Discussion}

In this study, we have laid out the methodology and critical steps to use reverse transfected cell arrays in microfluidic flowcells $[95,96]$ for the robust, quantitative screening of GPCR libraries.

Our first experiments were aimed at studying reverse transfection and expression efficiency and revealed considerable differences in protein expression levels between individual cells (see Video S2). These differences may partially derive from uneven deposition of DNA on the glass surface or random differences in DNA uptake between cells [188], but most likely mainly reflect the well-known effect of the variation in cell cycle within the cell population used during reverse transfection [175]. Before plasmid DNA is taken up by the nucleus it can be continuously broken down in the cytoplasm [176], and trafficking of plasmid DNA to the nucleus is only optimal during mitosis. As a result, the sub-population of cells transfected in their late S or G2 phases will transfer higher amounts of plasmid DNA to the nucleus during $M$ phase than those cells transfected during the G1 / G0 phases. Therefore, to generate a more homogeneous and high gene expression, one could either synchronize the cell cycle prior to reverse transfection $[189,190]$, or alternatively, add nuclease inhibitors during the reverse transfection process [191] to reduce plasmid breakdown. In our experiments we did not synchronize the cells because the transfected DNA was well expressed and allowed sufficient receptor readout in our cell line. However, with other cell lines, smaller spots or poor signals, it may be advisable to synchronize cells for higher and more homogeneous gene expression.

In large reverse transfected cell arrays, the variation in individual cell expression was captured in the variation of pixel intensities. To analyze these large cell arrays, we introduced thresholds to separate fluorescent pixels from background and over-exposed pixels. Resulting spots were defined as the collection of pixels with values between the upper and lower thresholds. Using this method we examined the optimal DNA concentration in the print solution by assessing the expression of fluorescent proteins and found a nonlinear increase in average fluorescence per spot with increasing total DNA concentration. Examination of the effect of DNA concentration in the print solution on protein expression revealed that, within a certain range, the total DNA concentration has a much greater influence on protein expression than the relative specific DNA concentration. The 10-fold 
increase in fluorescence signal that we observed could be accounted for almost entirely by the quantity of total DNA [192]. By contrast, when keeping the total DNA concentration constant, we observed a linear increase in fluorescence relative to the specific DNA concentration. A similar linear increase of fluorescent protein expression with constant total DNA was obtained by Woodruff et al. [84]. Overall the results indicate that, in the tested concentration range, the efficient nuclear uptake of DNA is controlled more by the total DNA concentration than by the specific DNA concentration, thus suggesting strong differences in DNA survival. Also other studies have shown that adding non-coding plasmid DNA can improve expression efficiency by serving as a protection buffer against nucleases in the cytoplasm [192].

The goal of our experiments was to develop a versatile microfluidic system to screen GPCRs libraries by sequentially injected samples. Co-transfection with the fluorescent cytosolic calcium ion sensor Yellow Cameleon YC3.6 enabled the monitoring of transient calcium ion changes and discrimination of transfected and non-transfected cells on the cell array. Additionally, co-transfection allowed us to tune expression of both genes independently and to analyse the effect of receptor coding DNA concentration separately from the calcium sensor concentration. We observed that the lowest concentration of specific, NK1-encoding DNA, $1 \%$ of total DNA $(0.3 \mathrm{ng} / \mu \mathrm{l})$, still yielded sufficient gene expression for a significant increase in intracellular calcium in response to 500 pM SP. This suggests that sufficient numbers of intact plasmids reach the cell nucleus to facilitate NK1 gene expression even at those ratios. Studies have shown that during a transfection event, individual cells can take up about $10^{5}$ to $10^{6}$ copies of a plasmid [178]. However, as discussed earlier, plasmids also need to reach the nucleus in order to be expressed, but are prone to rapid degradation by nucleases naturally present in the cytoplasm [176]. The nuclear plasmid copy number was determined by Cohen et al. in 2009 [177] to range 40-fold from 75 to 3000 copies per nucleus, and they also demonstrated a direct correlation between nuclear plasmid concentration and gene expression. We used a 50-fold difference in NK1 receptor DNA concentration in the spot mixes and found that the lowest concentration NK1 coding DNA resulted in only a 4-fold decrease in calcium ion signal. It will be interesting in the future to use the reverse transfected cell array format to evaluate the effect of receptor expression level on the signalling function of other GPCRs or ion-channels, because the highest expression level may not be optimal for gene function. Similarly, these arrays are highly suitable to evaluate permutated cofactor genes and to discover orphan receptor function.

We subjected the NK1 receptor cell array to sequential injections of ligand and monitored the calcium responses. This revealed that spots with higher amounts of NK1 plasmid DNA and higher response maxima were subject to losing signal strength more rapidly compared to spots with lower amounts of NK1 plasmid DNA. Such declining receptor responses are generally known for GPCRs and are thought to be caused by receptor internalization and recycling after (over) activation [47, 139]. Scarce endogenous co-factors of the signaling 
pathway (G-proteins, beta-arrestins, etc), specifically associated with the activated receptors may be depleted more rapidly in the presence of a high receptor density during this process of internalization, and possibly explain our observations $[67,193]$. Therefore, by controlling the GPCR DNA concentration in reverse transfection, the sensitivity and robustness (a reproducible response to repeated challenges) of the receptor response can be optimized. This is shown in the last experiment where we have exposed two different receptors sequentially to alternating samples. These receptors proved to be highly specific for their ligand since no cross reaction was observed and responses were highly reproducible at the given non-saturating concentrations. The analysis required an approach where inter-spot variation was eliminated, focusing only on comparisons within the time profile of individual spots. The mixed-model approach taken here, where each spot has a random intercept, provides a simple and natural way of fitting these data, demonstrating reproducible responses for sequential injected samples.

Overall, the GPCR library screening platform presented here is complementary to multiwellbased systems as it is simple, fast and efficient for screening receptor libraries with small amounts of ligand (see Video S1). It is perhaps less suitable for screening compound libraries because it depends on sequential rather than parallel injection. Previously, reverse transfection arrays encoding GPCR libraries were printed in static multiwell formats $[81,85]$ and not yet as part of a microfluidic platform. We believe that the microfluidic properties and the potentially very large size of the arrays creates significant potential to make this system a useful high throughput and high content screening tool for GPCR and ion-channel libraries. A single sample exposure of 50-150 microliters for 30 seconds (depending on flowspeed and flowcell volume) can reach hundreds of different receptors in parallel. This screening platform is in that sense complementary to systems with multiple microchambers that are independently addressed [84, 181]. On-chip corrections for fluorescence quenching and sample fluorescence could be implemented with Cameleon mutants that do not bind calcium for example. Receptor saturation levels could be monitored by including calibration injections. Cameleon expressing control spots lacking recombinant receptors can be used to monitor the build-up of intracellular calcium in time and endogenous cell responses and other artefacts affecting the intracellular calcium ion concentrations.

\section{Conclusion}

In conclusion, this paper has studied the main parameters for optimization of reverse transfection cell arrays for the detection of GPCR signaling in a microfluidic system. We observed an intrinsic, large variation in expression levels between the cells of one array spot. On optimization of protein expression efficiency we found that the total DNA concentration is a critical parameter to achieve high expression levels. Functional co-transfections were performed showing a fine-tuning of receptor expression levels by changing the input 
plasmid DNA concentration. Concentrations down to $1 \%$ of total DNA still yielded specific receptor responses. Finally, repeated receptor challenges showed a highly reproducible signal. These findings provide a confident outlook on introducing this system as a novel high throughput GPCR library screening tool.

\section{Supplementary Materials}

The following are available online at www.mdpi.com/1424-8220/18/2/602/s1: Video S1. Animation of the preparation, measurement and analysis of GPCR arrays. Video S2. Reverse transfection time laps movie. Supplemental Figure S1. Overview of slide preparation and slide measurement. Figure S2. Printed DNA array before and after reverse transfection. Figure S3. Fluorescence image of a large reverse-transfected cell array.

\section{Acknowledgments}

This work was supported by NanoNextNL, a micro- and nano-technology consortium of the government of the Netherlands and 130 partners. Imaging facilities were offered by the Wageningen Light Microscopy Centre and the Cell Biology and Immunology Group of Animal Sciences at Wageningen University and Research

\section{Author Contributions}

M.R., M.G.L.H. and H.A.V. conceived of and designed the experiments. M.R. performed the experiments and analysed the data. M.G.L.H. and M.S.v.L. contributed to the development of the data analysis tools. N.C.A.d.R. provided research equipment and technical support. R. W. developed statistical models. M.R. and M.A.J. wrote the paper, and R.F.W. and R.D.H. performed substantive revisions.

\section{Conflicts of Interest}

The authors declare no conflict of interest. The founding sponsors had no role in the design of the study; in the collection, analyses or interpretation of data; in the writing of the manuscript; nor in the decision to publish the results. 


\section{Supplementary Figures}
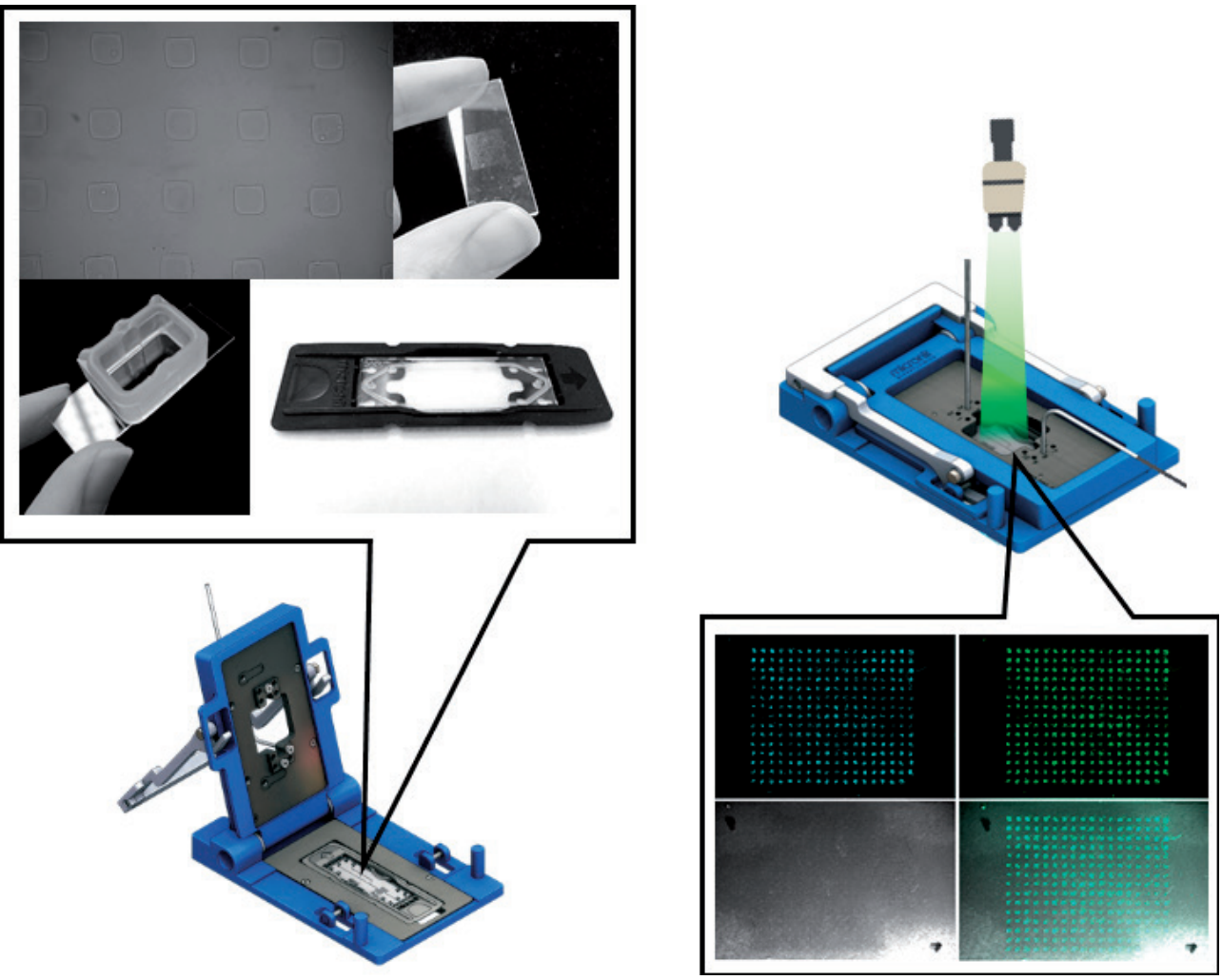

Figure S1, Overview of slide preparation and slide measurement. A, Uniform printed array (top left and right) with spot size of $200 \mu \mathrm{m}$ and a spot spacing of $600 \mu \mathrm{m}$. A flexiPERM chamber for cell growth mounted on the array (lower left), and an assembled flowcell (lower right) mounted in the flowcell holder below. B, Imaging setup using a stereo-fluorescence microscope that records both CFP and YFP FRET emissions of the whole cell array. The bright field image shows the uniform cell layer of HEK293 cells after growth in the flexiPERM chamber. 

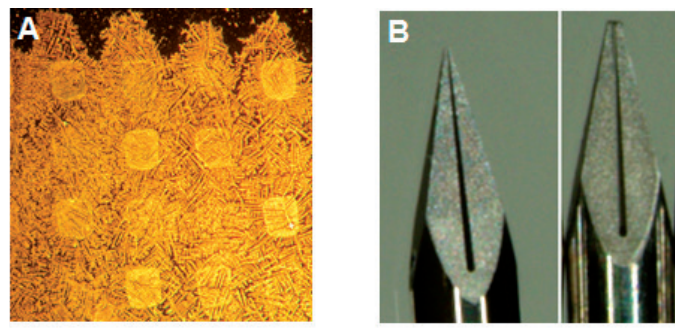

\begin{tabular}{|l|l|l|l|}
\hline 30 & 20 & 10 & 40 \\
\hline 10 & 40 & 30 & 20 \\
\hline 30 & 20 & 10 & 40 \\
\hline 10 & 40 & 30 & 20 \\
\hline
\end{tabular}

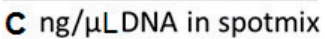

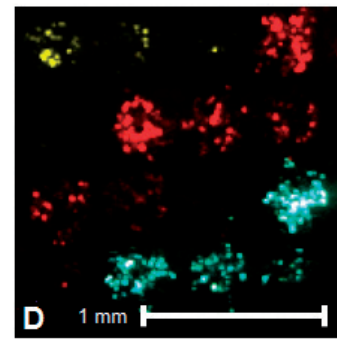

Figure S2, Printed DNA array before and after reverse transfection. A, Printed DNA array after effectene treatment and drying. Spot size is $200 \mu \mathrm{m}$, spot spacing is $400 \mu \mathrm{m}$. B, Hollow needles used to print spots. The sharp tip on the left yielded spots of about $100 \mu \mathrm{m}$ and the blunted one on the right yielded spots of $200 \mu \mathrm{m}$. C, Total DNA concentration in $\mathrm{ng} / \mu \mathrm{l}$ for the corresponding spots of $\mathbf{A}$. D, Array of A 24 hours after reverse transfection with HEK293 cells. Spots expressed the fluorescent proteins CFP, RFP or YFP. The spacing of $400 \mu \mathrm{m}$ risked spot cross-contamination therefore an interspot distance of $600 \mu \mathrm{m}$ was used in all other arrays.

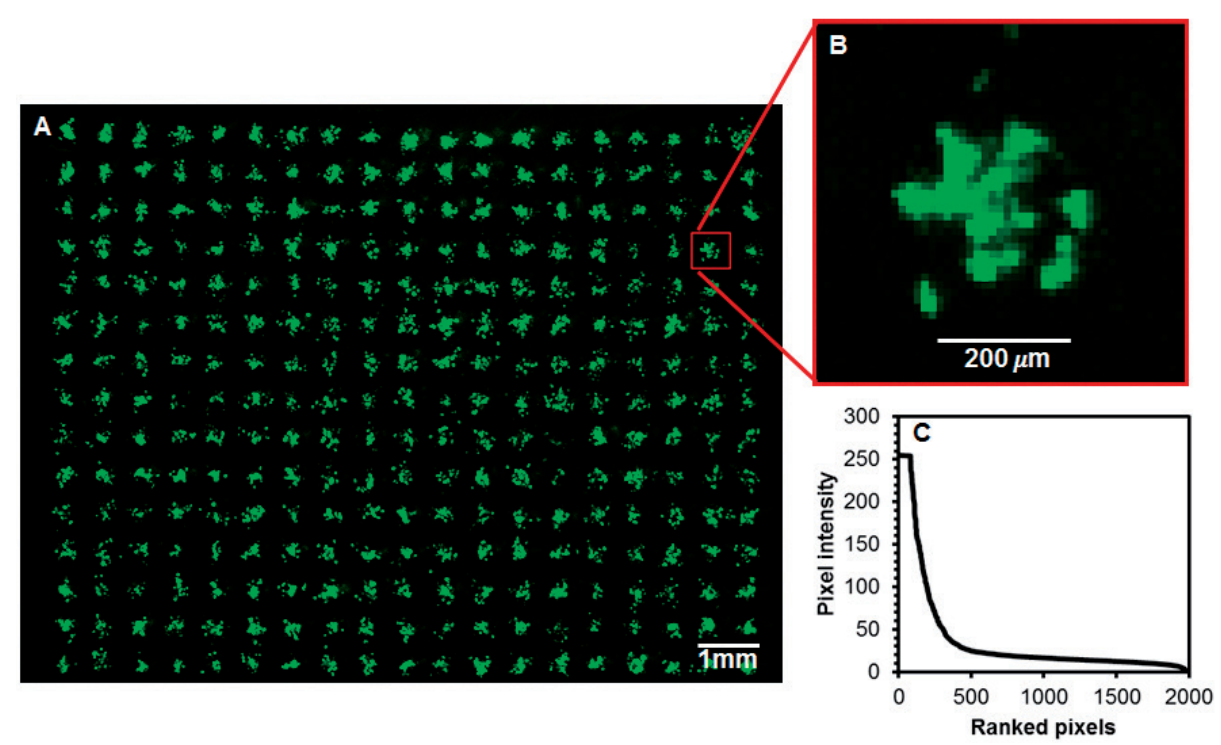

Figure S3, Fluorescence image of a large reverse transfected cell array. A, Large cell array of $15 \times 19$ $(9 \times 10.8 \mathrm{~mm})$ spots with diameters of $200 \mu \mathrm{m}$ and a spacing of $600 \mu \mathrm{m}$. B, Enlarged section( $\sim 45 \times 45$ pixels) where one pixel represents $\sim 11 \mu \mathrm{m}$ (no binning). C, Pixels of section B ranked from highest to lowest intensity. The distribution shows some saturated pixels at intensity of 256 , fluorescent protein expressing cells between intensity $\sim 20$ and 256 , and background pixels at intensities $<20$. 

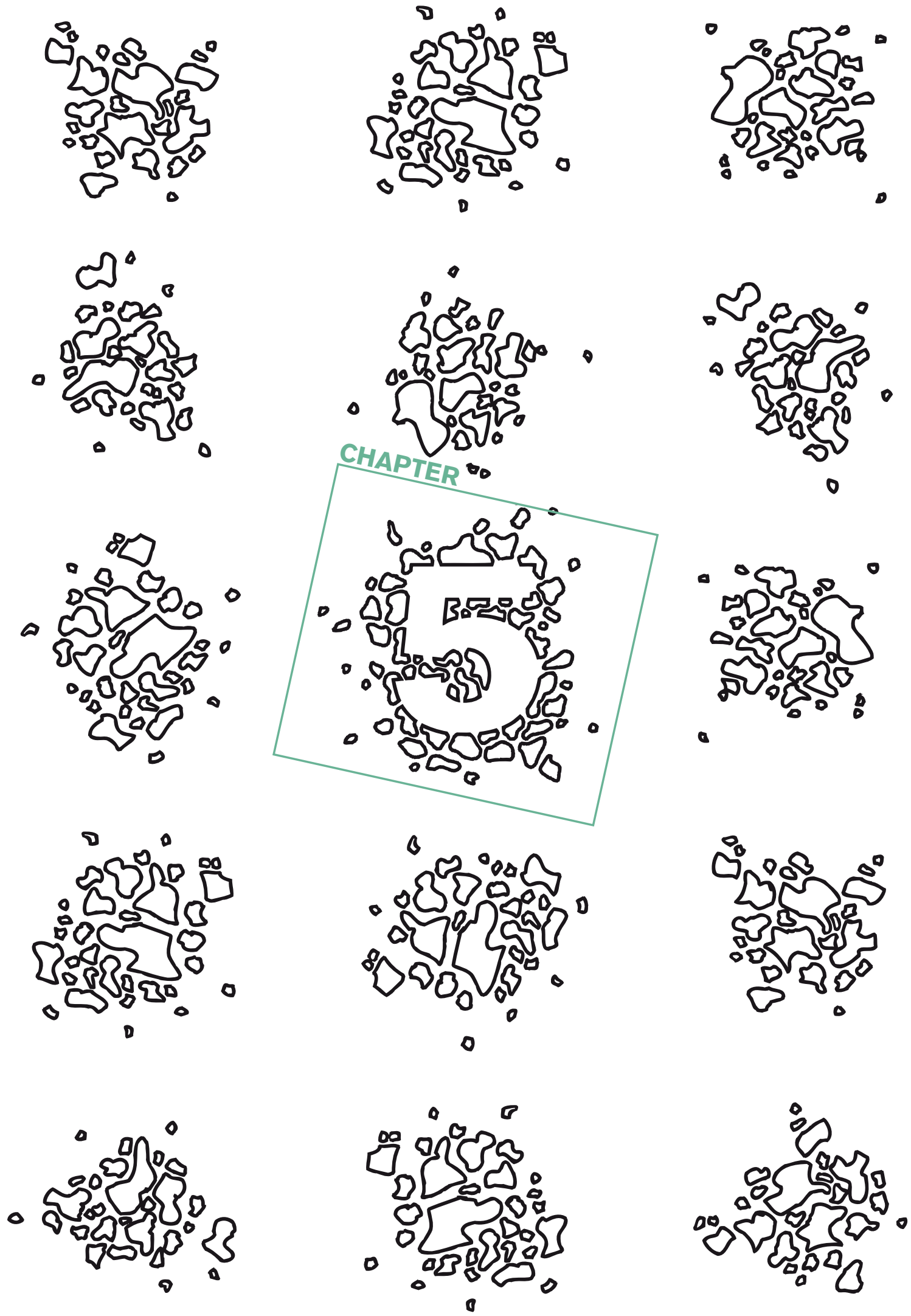


\section{The effect of calcium buffering and calcium sensor type on the sensitivity of an array-based bitter receptor screening assay}

Margriet Roelse, Ron Wehrens, Maurice G.L. Henquet, Renger F. Witkamp, Robert D. Hall and Maarten A. Jongsma 


\section{Abstract}

The genetically encoded calcium sensor protein Cameleon YC3.6 has previously been applied for functional $\mathrm{G}$ protein-coupled receptor screening using receptor cell arrays. However, different types of sensors are available, with a wide range in $\left[\mathrm{Ca}^{2+}\right]$ sensitivity, Hill coefficients, calcium binding domains and fluorophores, that could potentially improve the performance of the assay. Here, we compared the responses of three structurally different calcium sensor proteins (Cameleon YC3.6, Nano140 and Twitch2B) simultaneously, on a single chip, at different cytosolic expression levels and in combination with two different bitter receptors, TAS2R8 and TAS2R14. Sensor concentrations were modified by varying the amount of calcium sensor DNA that was printed on the DNA arrays prior to reverse transfection. We found that $\sim 2$ fold lower concentrations of calcium sensor protein, by transfecting 4 times less sensor-coding DNA, resulted in more sensitive bitter responses. The best results were obtained with Twitch2B, where, relative to YC3.6 at the default DNA concentration, a 4-fold lower DNA concentration increased sensitivity 60-fold and signal strength 5- to 10 -fold. Next we compared the performance of YC3.6 and Twitch2B against an array with 11 different bitter taste receptors. We observed a 2-8 fold increase in sensitivity using Twitch2B compared to YC3.6. The bitter receptor arrays contained 300 spots and could be exposed to a series of 18 injections within one hour resulting in 5400 measurements. These optimised sensor conditions provide a basis for enhancing receptomics calcium assays for receptors with poor $\mathrm{Ca}^{2+}$ signalling and will benefit future high throughput receptomics experiments. 


\section{Introduction}

Bitter taste receptors (T2Rs or TAS2Rs) are a class of G protein-coupled receptors, for which 25 subtypes have now been identified in the human genome. Some are broadly tuned (e.g. TAS2R10,-14,-46), whereas others are more specific (e.g. TAS2R3,-5,-9,-13,-20,-41,-45,-50). Next to this, there are also four still remaining with an orphan status (TAS2R19,-42,-45,-60) [194]. Interestingly, their expression and downstream signalling is not limited to the oral cavity and has been found to include other parts of the gastro-intestinal tract, respiratory and genitourinary systems, brain and immune cells $[16,195]$. These findings suggest roles beyond taste, which is further fuelling the search for endogenous and exogenous ligands.

In screening assays used thus far, a number of bitter receptors have shown poor signals that could be related to variable functional expression on the cell membrane [32, 196-199]. Receptors with lower functional expression may suffer from poor transport to or rapid internalization from the cell membrane [197], may have poor glycosylation [198] and with certain levels of constitutive activity may become phosphorylated by receptor kinases that target them for $\beta$-arrestin internalization and may become generally exhausted for signalling [55]. Also, when comparing cytosolic calcium signalling of neuropeptide receptor 1 (NK1) to the relatively strongly responding bitter receptor 8 (TAS2R8), we found that the signal amplitude for TAS2R8 was approximately 3-fold lower than for the NK1 receptor [100]. When signals are low, it is important to use a probe that is the most sensitive in the resting range of cytosolic calcium levels. Previously, in reverse transfected arrays with bitter receptors, calcium assays were performed using the Cameleon YC3.6 sensor protein, because of its wide dynamic range (5-6 fold change in the ratio of YFP/CFP upon $\mathrm{Ca}^{2+}$ binding in vitro) and brightness [131, 200]. The arrays were typically printed using two plasmids at identical concentrations; one encoding the G protein coupled receptor (GPCR) and one the calcium sensing protein. However, the effect of the concentration and type of sensor protein on the sensitivity and signal strength of the assay was not previously considered.

Since the first use of Cameleon calcium sensors [125], many variants of this ratiometric calcium probe have been described that are also based on the response of the FRET pair CFP and YFP [201, 202]. The palette of probes involves various modifications: The CFP and YFP proteins have been optimized to enhance their spectral properties [203], the YFP protein has been circularly permutated [131], the linker between CFP and YFP has been varied in length [204], and the calcium binding modality has been changed from calmodulin to troponin [205]. Ratio-imaging of YFP and CFP is advantageous because it is, in principle, independent of the expression level of the calcium sensor, and thus a direct reporter of the actual calcium concentration. For a receptomics platform this is relevant because the genetically encoded calcium sensor is transiently expressed in a receptor cell array which results in highly variable expression levels between cells within a spot. The genetically 
encoded property of this fluorescent probe further allows the identification of transfected cells prior to the start of the experiment and the correct positioning of the array under the microscope. Fluorescent calcium sensor proteins like the GECO probes [201] only show significant fluorescence emission upon calcium increase. This yields very low fluorescent signals of the array prior to the start of a GPCR activation experiment, which are more difficult to focus, position and analyse.

In this study we aimed to evaluate ratiometric probes with a calcium affinity that would ensure sufficient sensitivity for relatively minor calcium transients of bitter taste receptors above the resting levels of 100 nM in HEK293 cells [206]. Cameleon YC3.6 [131, 200, 204], Nano140 [204] and Twitch2B [205] have $K_{d}$ values of $\sim 140-250 n M$ that fit this criterion in principle, but these probes are distinctly different: YC3.6 and Nano140 both include a modified calmodulin domain, but with different cooperative binding and dissociation constants, and Twitch2B contains a troponin domain with only one calcium binding pocket and a more linear calcium binding curve. An added advantage of the use of troponin over calmodulin is that it does not potentially interact with host cell calmodulin binding proteins [202]. Next to sensor type, we also aimed to evaluate the effect of the cytosolic sensor concentration, because a high sensor concentration may increase signal-to-noise levels, but may also result in a lower signal intensity because of calcium buffering $[200,207]$.

\section{Materials and methods}

\section{Expression vectors}

Reverse transfected cell arrays were prepared and analysed as previously described in Roelse et al [100]. The genes encoding TAS2R1, TAS2R3, TAS2R4 (both FVS and SLN variants), TAS2R8, TAS2R10, TAS2R14, TAS2R16, TAS2R38 (both PAV and AVI variants), TAS2R39, TAS2R43 and TAS2R46 (see suppl. Table 1 for sequence information) were obtained from genomic DNA of HEK293 cells by PCR amplification and were cloned into pcDNA3 containing the N-terminal sstr3 tag (gift from Dr. Wolfgang Meyerhof, German Institute of Human Nutrition Potsdam-Rehbrücke, Germany). Plasmid, pcDNA YC3.6 was obtained from Prof. Roger Tsien (UC San Diego). Yellow Cameleon-Nano140 pcDNA3 was a gift from Takeharu Nagai (Addgene plasmid \# 51966), Twitch-2B pcDNA3 was a gift from Oliver Griesbeck (Addgene plasmid \# 49531)

\section{Receptomics assay}

DNA arrays comprising $15 \times 19$ spots were printed and processed as previously described [100]. Reverse transfected cell arrays were prepared using HEK293 cells stably transfected with $\mathrm{Ga}_{16 \mathrm{GUST44}}$ (a gift from Dr. Takashi Ueda, Nagoya City University, Nagoya, Japan). At 48 hours after transfection, the cell arrays were taken from the incubator, washed and 
incubated in assay buffer for 1 hour prior to the measurements. Spots then were typically composed of 25-50 cells and imaged by a similar number of pixels. All measurement series were performed using a $\sim 150 \mu$ l flowcell in the flowcell holder (Micronit Microfluidics B.V., Fluidic Connect PRO Chip Holder.) The assay buffer C1 ( $\mathrm{NaCl} 130 \mathrm{mM}, \mathrm{KCl} 5 \mathrm{mM}$, Glucose 10 $\mathrm{mM}, \mathrm{CaCl}_{2} 2 \mathrm{mM}$, HEPES $10 \mathrm{mM}$ at pH 7.4) was set at a continuous flow of $300 \mu \mathrm{l} / \mathrm{min}$ across the array and sample injections were performed with a volume of $150 \mu$ l (Waters 2795 autosampler). Agonists used in the injections were ATP (Sigma A6419), Chloramphenicol (Duchefa C0113.0100), Aristolochic acid (Sigma A5512), Denatonium benzoate (Wako 04623561), Picrotoxinin (Sigma P8390), PROP (Sigma P3755) and D-Salicin (Wako 199-00083). The arrays were imaged by a Leica fluorescent stereo microscope (Leica M205FA with DFC 345 FX camera and 2.0x PlanApo objective with NA 0.35) fitted with a 0.32× C-Mount and filters for CFP (ET CFP 10447409, excitation 436/20 and emission 480/40) and YFP FRET (ET FRET 10450566, exc. 436/20 and em. 535/30). Lamp intensity (Osram, HXP-R 120W/45C VIS) was set at maximum, and the exposure time was $400 \mathrm{~ms}$.

\section{Rmin and Rmax measurement}

The $R_{\min }$ and $R_{\max }$ signals were measured in a reverse transfected cell array containing mock transfected spots with either Twitch2B or YC3.6, according to the method described in [126]. In short, the array was equilibrated applying a $200 \mu \mathrm{l} / \mathrm{min}$ flow of $\mathrm{C} 1$ buffer for 10 minutes. First a blank injection of $300 \mu \mathrm{l} 1$ buffer was performed followed by a $300 \mu$ injection with $\mathrm{R}_{\text {min }}$ buffer ( $\mathrm{C} 1$ buffer without $\mathrm{CaCl}_{2}$ but supplemented with 3mM EGTA and $5 \mu \mathrm{M}$ ionomycin (Sigma 10634) adjusted to $\mathrm{pH} 7.4$ using $\mathrm{NaOH}$ ). One minute after the $\mathrm{R}_{\text {min }}$ injection, when the $R_{\text {min }}$ buffer exposure was maximal, the flow was stopped for 15 min to allow the cells to become fully calcium ion depleted. Next, the flow was restarted replacing the $R_{\min }$ buffer with the $\mathrm{C} 1$ buffer and cells were equilibrated again in the $\mathrm{C} 1$ buffer for $10 \mathrm{~min}$. Subsequently, $300 \mu$ l of $\mathrm{R}_{\max }$ buffer ( $\mathrm{C} 1$ buffer with instead $5 \mathrm{mM} \mathrm{CaCl}_{2}$ and $5 \mu \mathrm{M}$ lonomycin) was injected into the flow and the flow was again stopped after $1 \mathrm{~min}$. Cells were then again left for 10 min to become fully calcium saturated allowing the measurement of $R_{\max }$.

Calcium affinity curves were calculated using a Hill coefficient calculation modified from [208]:

$\mathrm{F}(\mathrm{S})=\mathrm{R}_{\text {min }}+\left(\Delta_{\max } * \mathrm{~S}^{\mathrm{n}}\right) /\left(\mathrm{K}_{\mathrm{d}}{ }^{\mathrm{N}} * \mathrm{~S}^{\mathrm{n}}\right)$

$\mathrm{R}_{\text {min }}=$ minimal YFP/CFP ratio

$\Delta \max =$ difference between $R_{\text {min }}$ and $R_{\text {max }}$

$\mathrm{S}=$ calcium concentration $\left[\mathrm{Ca}^{2+}\right]$

$\mathrm{n}=$ Hill coefficient

$\mathrm{K}_{\mathrm{d}}=\mathrm{EC}_{50}$ calcium value of the sensors

Hill coefficient and $K_{d}$ values were used from literature sources. Twitch2B has a $K_{d}$ of 200 $\mathrm{nM}$ and Hill coefficient of 1.3 according to [205]. For YC3.6 conflicting values have been 
published. The initial publication states a $\mathrm{K}_{\mathrm{d}}$ of $250 \mathrm{nM}$ and a Hill coefficient of 1.7 [131]. However, a more thorough study performed by Horikawa et al. [204] showed a bi-phasic calcium affinity curve for YC3.6 with a high affinity range of $K_{d}$ of $215 \mathrm{nM}$ and Hill coefficient of 3.6, and a low affinity range of $\mathrm{K}_{\mathrm{d}}$ of $780 \mathrm{nM}$ and Hill coefficient of 1.2. Here, we used the high affinity range of $\mathrm{K}_{\mathrm{d}}$ of $215 \mathrm{nM}$ and Hill coefficient of 3.6 to plot a comparative curve.

\section{Data analysis}

The analysis of data from the receptor cell arrays was performed as described in Wehrens et al [105]. In short, FRET images for the CFP and YFP channels were recorded and converted, using the CellProfiler software package [185], into raw CFP and YFP intensity values. Spots with less than 15 fluorescent pixels and spot types with fewer than 5 replicates were removed from the data analysis. After smoothing and interpolation to remove the differences in timing between the CFP and YFP measurements, spot signals were calculated as the ratio of the CFP and YFP values. These signal peak heights associated with individual injections were calculated as the difference between start and maximal ratio value in the spot signal within a time window of 30 cycles (just under two minutes). A representative example is shown in the supplementary data to Wehrens et al [105]. These signal peak heights, after log-scaling were then used in a mixed model with the injection type as fixed variable, and spot number as random variable. This greatly improves statistical power, since the between-spot variation is eliminated. Results presented in the figures consist of treatmentversus-control contrasts, with the blank injection as the control, after back-transformation to the original scales. This leads to coefficients that should be interpreted as multiplicative effects: a value of 1.1 should be interpreted as a $10 \%$ increase in response compared to the reference. A value of 1.0 indicates no difference to the reference. Depending on the goal or characteristics of the experiments, different choices for reference injections are possible: like blank injection(s) or a sample contrast as in Wehrens et al. 2019.

\section{Results}

\section{Effect of sensor type and relative sensor concentration}

On a receptor cell array, three sensor types (YC3.6, Nano140 and Twitch2B) and two receptor types (TAS2R8 and TAS2R14) were combined in different configurations. The sensor plasmids were printed at three concentrations (8, 16 and $25 \mathrm{ng} / \mu \mathrm{l})$, while keeping the receptor coding DNA (25 ng/ $\mu$ l) concentration constant. Empty vector DNA was added to maintain the same total DNA concentration $(50 \mathrm{ng} / \mu \mathrm{l})$. The receptor cell array was exposed to increasing concentrations of a mixture of chloramphenicol and picrotoxinin, selective ligands for TAS2R8 and TAS2R14, respectively. The experiment was repeated twice using different cell arrays. Figure 1 shows an example of the raw traces of an injection series for TAS2R8 and TAS2R14, representing all three sensor types at a concentration of $16 \mathrm{ng} / \mu \mathrm{l}$. 

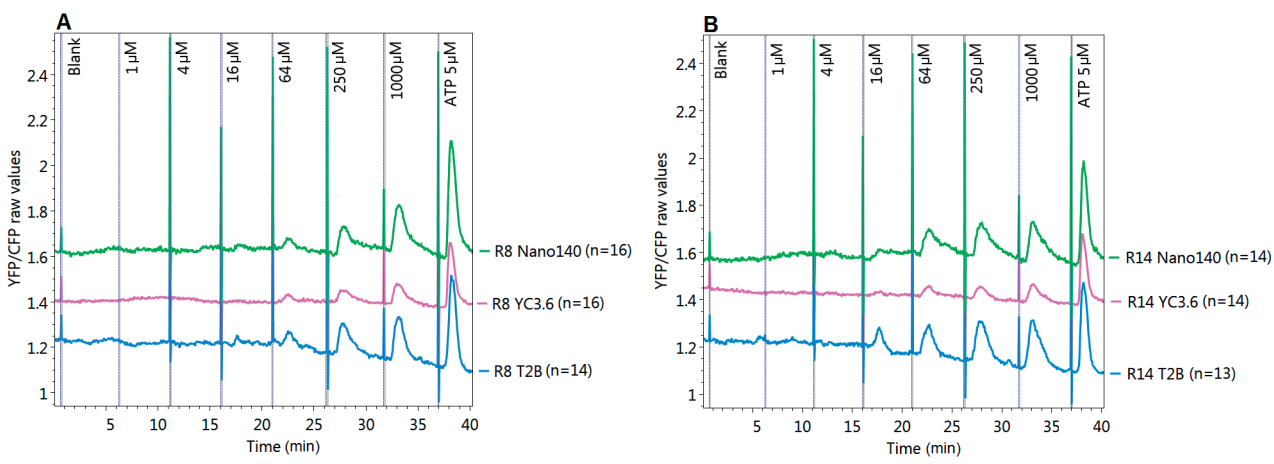

Figure 1, Averaged raw traces of TAS2R8 (A) and TAS2R14 (B) in an injection series of increasing chloramphenicol and picrotoxinin concentrations (array 2). Samples were injected sequentially at intervals of 5 minutes and with an exposure time of approx. 30 seconds. The ligand concentrations were as indicated in the chart. The lines represent averages of 13-16 replicated spots as indicated in the legends of the charts. In this example the three sensors were printed at a concentration of $16 \mathrm{ng} /$ $\mu$ I DNA.

After the blank injection, response peaks increased with increasing ligand concentrations for all three sensor types and for both bitter taste receptors. The final injection was a positive control injection with ATP at $5 \mu \mathrm{M}$. The purinergic receptors, endogenously expressed in the HEK293 host cell, are activated by ATP showing a clear positive control peak for all spot types. An additional 12 curves were obtained in parallel for the other sensor concentrations. The results are summarized in 18 replicated dose-response curves derived from the two arrays in $2 \times 40$ min (Figure 2). These response curves, including $95 \%$ confidence intervals, are shown in suppl. Figure 1.

The curve values represent estimates of treatment-versus-control contrasts with respect to the $1 \mu \mathrm{M}$ agonist injection, the lowest sample dilution which does not evoke a specific response signal. In this case, the injection with the lowest concentration was chosen as the reference since the blank injection in one of the arrays gave an artefact response, probably due to inadequate pre-washing of the auto sampler. Spot-based contrasts were determined to reduce the variation between the spots. The red and blue curves represent replicated experiments using two different arrays. The results for the two arrays are in good agreement, taking into account the width of the confidence intervals shown in Suppl. Figure 1. In both experiments with TAS2R8, the Twitch2B sensor at a gene dose of $8 \mathrm{ng} /$ $\mu \mathrm{l}$ gave the highest signal and sensitivity. For TAS2R14, with responses double those of TAS2R8, the effects were less pronounced but nevertheless still visible.

The effects of sensor type and sensor concentration are summarized in Table 1. Responses are based on treatment-versus-control contrasts compared to a non-response or blank treatment ( $1 \mu \mathrm{M}$ agonist in this case): a value of 1.1 should be interpreted as a ten percent increase in response compared to the reference, and a value of 1.0 indicates no difference. 

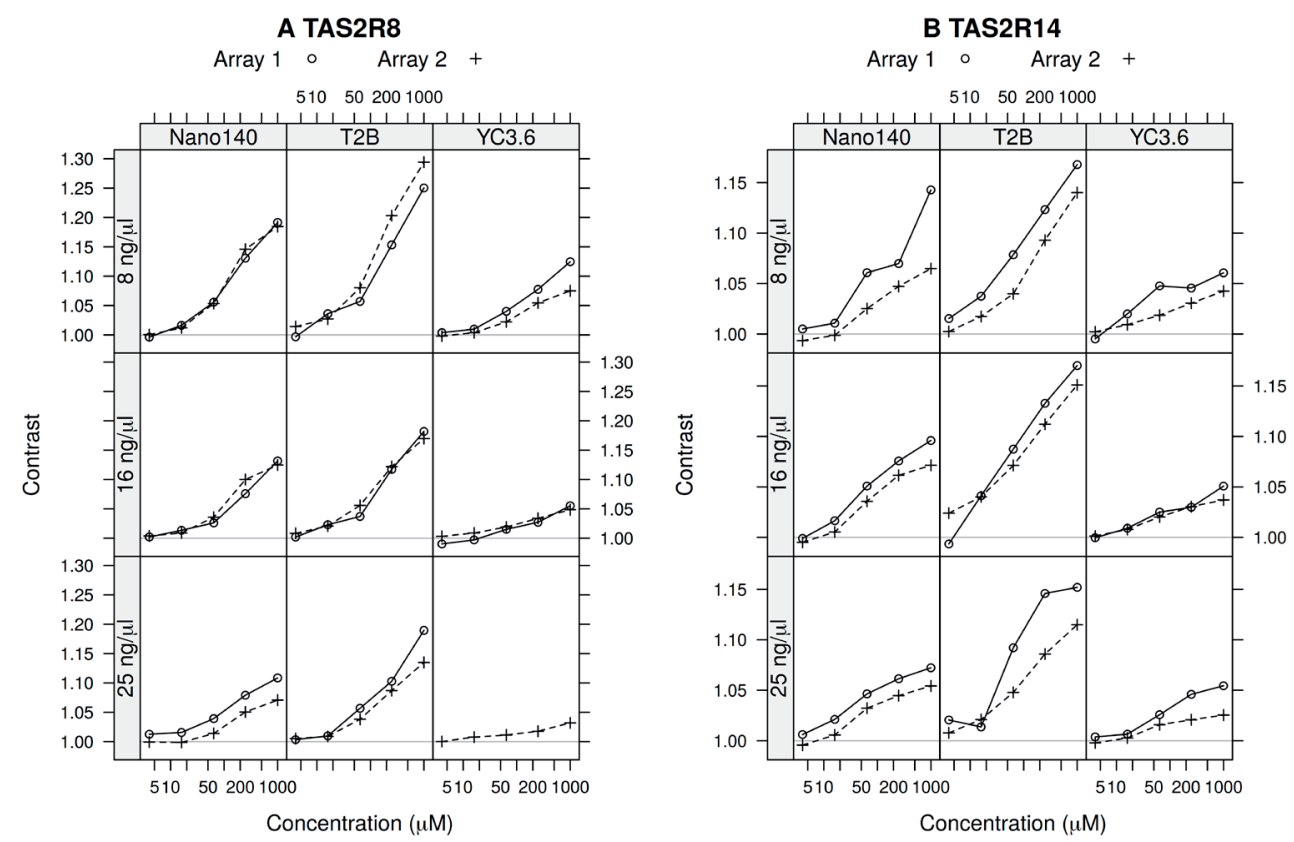

Figure 2, Response curves of TAS2R8 (A) and TAS2R14 (B) for stimulation with agonist mixture (chloramphenicol and picrotoxinin). (A) and (B) show agonist dose responses for the three sensor types at sensor gene dose of 8,16 and $25 \mathrm{ng} / \mu \mathrm{l}$. The two series represent two independent experiments. Response values represent estimates of treatment-versus-control contrasts with respect to the $1 \mu \mathrm{M}$ injection. T2B, Twitch2B.

Looking at a combined effect of sensor type and concentration, YC3.6 at $25 \mathrm{ng} / \mu \mathrm{l}$ (standard in [100]) compared to Twitch2B at $8 \mathrm{ng} / \mu \mathrm{l}$, when exposed to a $250 \mu \mathrm{M}$ ligand concentration, results in an 11-fold signal increase from 0.018 to 0.203 for TAS2R8 and a 4-5 fold increase for TAS2R14 (0.021 to 0.093). The sensitivity increase is up to 60 -fold with lowest significant detection at $16 \mu \mathrm{M}$ instead of $1000 \mu \mathrm{M}$. Splitting the sensor and concentration effects, it is clear that all probes benefit 4-15 fold from the reduced probe concentration and that Twitch2B is generally up to 4-fold more sensitive than the other two probes.

\section{Comparing YC3.6 and Twitch2B sensors}

The striking improvement in response amplitude and sensitivity for the bitter receptors TAS2R8 and TAS2R14 using Twitch2B instead of YC3.6, led us to investigate whether this also held up for other members of the bitter taste receptor family. To evaluate this, an array with 11 different bitter taste receptor genes combined with both sensors at a gene dose of $8 \mathrm{ng} / \mu \mathrm{l}$ was prepared. The array consisted of the bitter receptors TAS2R1, TAS2R3, TAS2R4 (both FVS and SLN genotypes), TAS2R8, TAS2R10, TAS2R14, TAS2R16, TAS2R38 (both PAV and AVI genotypes), TAS2R39, TAS2R43 and TAS2R46 M228L. Two controls were present on the arrays: mock transfected spots to observe and correct for any TAS2R-independent host 
Table 1, Effects of sensor type and concentration. The most optimal and least optimal conditions are marked in bold.

\begin{tabular}{|c|c|c|c|c|}
\hline & \multicolumn{2}{|c|}{ TAS2R8 } & \multicolumn{2}{|c|}{ TAS2R14 } \\
\hline & Lowest detection* & $\begin{array}{c}\text { Response at } \\
250 \mu \mathrm{M}^{* *}\end{array}$ & Lowest detection & $\begin{array}{l}\text { Response at } \\
250 \mu \mathrm{M}^{* *}\end{array}$ \\
\hline YC3.6 $8 \mathrm{ng} / \mu \mathrm{l}$ & $64 \mu \mathrm{M}$ & 1.054 & $250 / 64 \mu \mathrm{M}$ & 1.031 \\
\hline YC3.6 $16 \mathrm{ng} / \mu \mathrm{l}$ & $250 / 1000 \mu \mathrm{M}$ & 1.034 & $250 / 1000 \mu \mathrm{M}$ & 1.030 \\
\hline YC3.6 $25 \mathrm{ng} / \mu \mathrm{l}$ & $1000 \mu M$ & 1.018 & $1000 / 250 \mu M$ & 1.021 \\
\hline 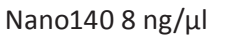 & $64 \mu \mathrm{M}$ & 1.146 & $250 / 64 \mu \mathrm{M}$ & 1.047 \\
\hline 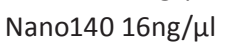 & $64 / 250 \mu \mathrm{M}$ & 1.100 & $64 \mu \mathrm{M}$ & 1.061 \\
\hline 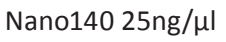 & $250 / 64 \mu \mathrm{M}$ & 1.051 & $64 \mu \mathrm{M}$ & 1.045 \\
\hline Twitch2B 8ng/ul & $16 / 64 \mu M$ & 1.203 & $64 \mu \mathrm{M}$ & 1.093 \\
\hline 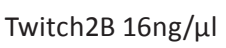 & $64 \mu \mathrm{M}$ & 1.122 & $16 \mu M$ & 1.112 \\
\hline 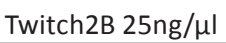 & $64 \mu \mathrm{M}$ & 1.087 & $64 \mu \mathrm{M}$ & 1.086 \\
\hline
\end{tabular}

*Detection threshold of agonist that gives a significant response in array 1 and 2, see suppl. Figure 1 . When thresholds are different both values of array 1 /array 2 are given.

**Response estimate of array 1 for exposure to $250 \mu \mathrm{M}$ agonist.

cell responses and YC- control spots. The YC- is a deletion mutant of YC3.6 ( $\triangle$ A332-S392) with fixed FRET between CFP and YFP to observe and correct for any optical effects of the samples during the fluorescence measurement. The receptors or empty vector (mock) were printed at a gene dose of $67 \mathrm{ng} / \mu$ l reaching a total DNA concentration of $75 \mathrm{ng} /$ $\mu \mathrm{l}$ that appeared optimal in a comparison of gene dosages from 50-150 ng/ $\mu \mathrm{l}$ total DNA (suppl. Figure 2). Receptor coding plasmid and total DNA concentration of the print mix was increased as a potential alternative way to improve the levels of functional receptor proteins present on the cell membrane [100]. The array contained 10 spot replicates of each bitter taste receptor, combined with either Twitch2B or YC3.6 at $8 \mathrm{ng} / \mu \mathrm{l}$.

For each array, two dose response experiments were performed by alternating agonist injections of pure compounds with increasing concentrations as shown in Figure 3 . In total, 3 arrays were used to prepare the dose response curves for 6 different agonists as presented in Figure 4. The agonist pairs were designed so that each would trigger a different receptor type. This resulted in a highly efficient throughput since in only three hours of measurement time we were able to generate 150 dose response curves and 12 mock or negative control curves (Suppl. Figure 3).

Since each array contained two separate experiments, treatment-versus-control contrasts could be calculated with respect to the same blank injection, making it possible to obtain all results in a single analysis. The mock control did not show a significant response signal to any of the agonist doses confirming that the receptor responses that were observed are bitter receptor specific. In Table 2 the results are summarized indicating a response range for each receptor-agonist combination with either Twitch2B or YC3.6 as calcium sensor. 

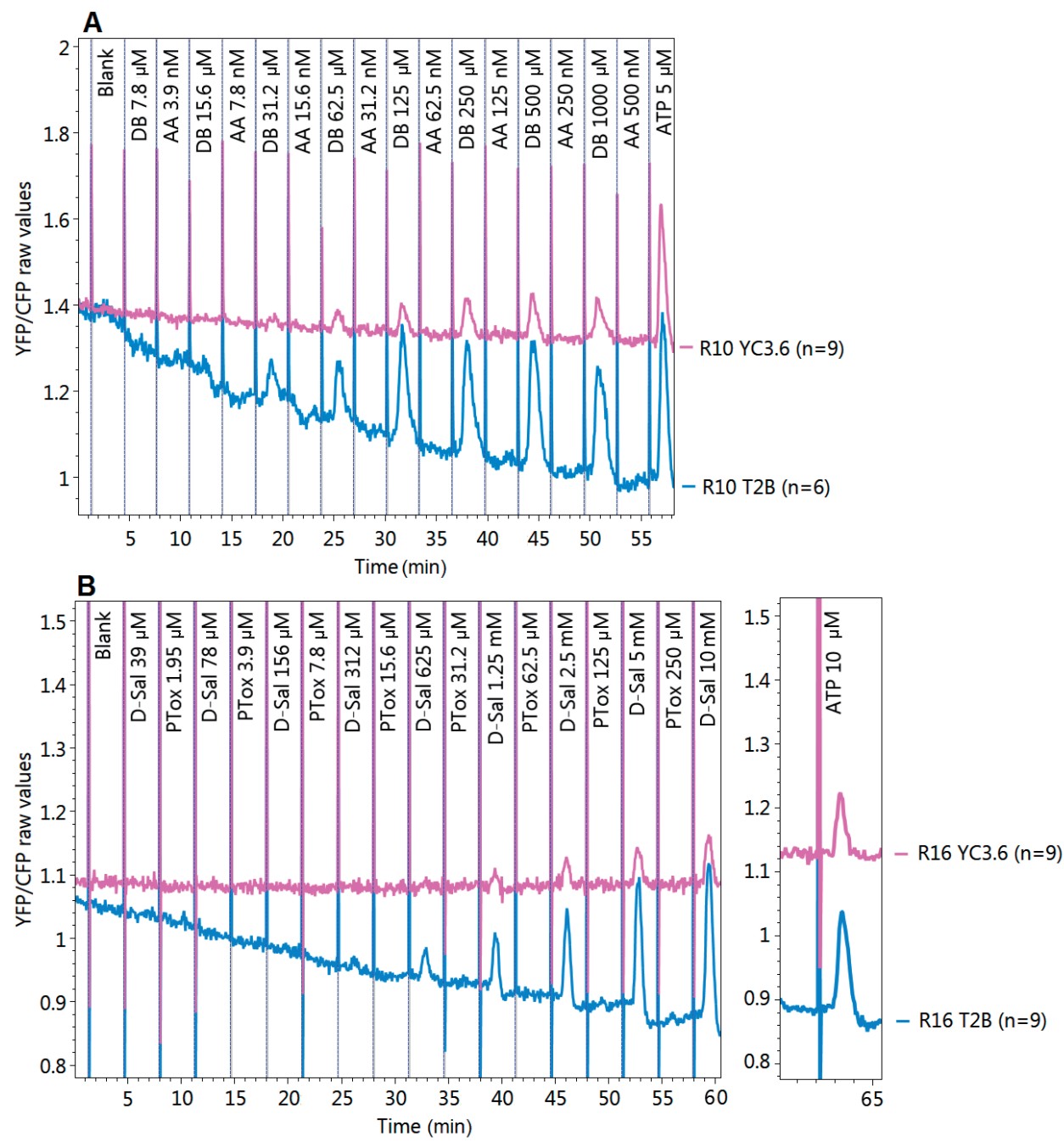

Figure 3, Averaged raw traces YFP FRET / CFP from (A) TAS2R10 and Denatonium Benzoate (DB) and (B) TAS2R16 and D-Salicin. Traces are shown for both Twitch2B (T2B) and YC3.6 sensor combination. Agonist injections were alternated every 3 minutes and agonist exposure was approx. 30 seconds. The lines represent averages of 6 to 9 replicated spots as indicated in the legends of the charts. AA, aristolochic acid, PTox, picrotoxinin, D-Sal, D-salicin, DB, denatonium benzoate.

Both sensor proteins were evaluated at similarly low DNA concentrations, but there is a 2-8 fold increase in sensitivity of Twitch2B compared to YC3.6. Some response signal exhaustion may have occurred in the series and the response peak height of the higher compound concentrations may be an underestimation. In Wehrens et al. 2019 we have shown how dosing order impacts the peak height. In general, a repeated sample injection protocol can be incorporated into the analysis model to estimate the global decline of signal. 


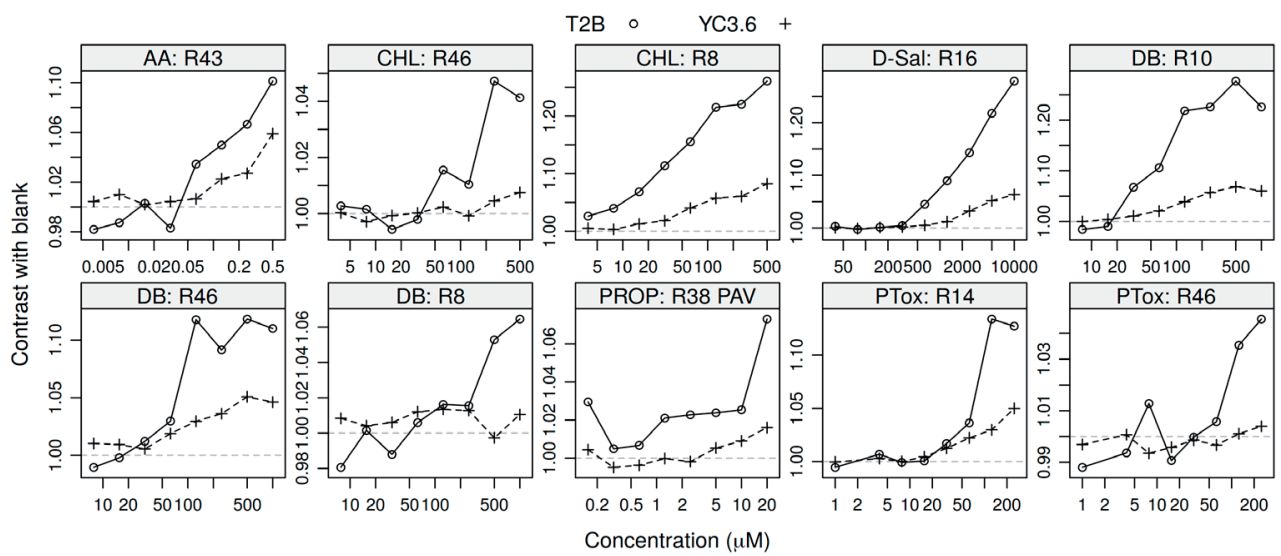

Figure 4, Response curves of Twitch2B (T2B, circles) and YC3.6 (marks) of the responding TAS2R receptor spots from the three experiments. The full set of responses are available in suppl. Figure 3. Response values represent estimates of treatment-versus-control contrasts with respect to the blank injection. AA, aristolochic acid, CHL, chloramphenicol, PTox, picrotoxinin, D-Sal, D-salicin, DB, denatonium benzoate and PROP, 6-n-propylthiouracil.

To check for metabolic exhaustion, a standard injection of ATP $5 \mu \mathrm{M}$ or $10 \mu \mathrm{M}$ was included at the end of each series. Such host cell responses triggered by ATP or Somatistatin-14 are normally used to check the vitality of the cells as they trigger the same $\mathrm{IP}_{3}$ pathway via PLC1-4 [209-211]. Any changes in ATP response may indicate negative effects of the exposure to the standard bitter compounds. Supplemental Figure 4 shows the ATP response contrasts to the blank for each spot-type on each array. Spot-types with less than 5 spots were removed from the analysis (see also Supplemental Figure 3). The spot-type specific ATP signals did not significantly differ between the arrays showing no signs of metabolic exhaustion in these experiments. It is however relevant to notice that generally each spot type displays a different ATP response level that is usually lower than the mock. This has been shown before in Wehrens et al. (2019) and suggests negative interactions between bitter receptors and calcium signalling presumably independent of specific triggers [105].

\section{Calcium response curves of YC3.6 and Twitch2B}

Twitch2B and YC3.6 have similar dissociation constants of 200 and 215-250 nM respectively, but they differ strongly in their Hill coefficient (1.3 vs 3.6). To visualize the effect of the differences in Hill coefficient in the predicted calcium response curves of YC3.6 and Twitch2B, we measured the $R_{\min }$ and $R_{\max }$ of Twitch2B and YC3.6 using the method described in [126] (Figure 5A), and used a Hill coefficient calculation [208] to estimate the response curves in relation to the calcium ion concentration (Figure $5 B$ ). The $R_{\min }$ buffer containing EGTA and ionomycin chelates all calcium ions resulting in an $R_{\min }$ ratio of 1.4 for YC3.6 and 0.8 for Twitch2B after about 15 minutes of exposure. Initially, after adding the $R_{\text {min }}$ buffer there was a spike in the ratio signal, showing the calcium depletion from the intracellular 
Table 2, Difference in sensitivity for the receptor/agonist combinations with either Twitch2B or YC3.6 sensor transfected at DNA concentration of $8 \mathrm{ng} / \mu \mathrm{l}$.

\begin{tabular}{|c|c|c|c|c|c|}
\hline Receptor & Agonist & $\begin{array}{l}\text { Response } \\
\text { range* of } \\
\text { Twitch2B }\end{array}$ & $\begin{array}{l}\text { Response } \\
\text { range* of } \\
\text { YC3.6 }\end{array}$ & $\begin{array}{l}\text { Fold increase } \\
\text { in sensitivity }\end{array}$ & $\begin{array}{l}\text { Reference EC50 } \\
\text { values }\end{array}$ \\
\hline \multirow[t]{2}{*}{ TAS2R8 } & chloramphenicol & 3.9-125 $\mu \mathrm{M}$ & $31.2-500 \mu \mathrm{M}$ & 8 & n.d. \\
\hline & denatonium benzoate & $250-1000 \mu \mathrm{M}$ & - & & n.d. \\
\hline TAS2R10 & denatonium benzoate & $15.6-125 \mu \mathrm{M}$ & $125-500 \mu \mathrm{M}$ & 8 & $59 \mu \mathrm{M} \pm 30[212]$ \\
\hline TAS2R14 & picrotoxinin & $31.2-250 \mu \mathrm{M}$ & $62.5-250 \mu \mathrm{M}$ & 2 & $18 \mu \mathrm{M}$ [213] \\
\hline TAS2R16 & D-salicin & $0.312-10 \mathrm{mM}$ & $1.25-10 \mathrm{mM}$ & 4 & $\begin{array}{l}1.2 \mathrm{mM}[214], 1.1 \\
\mathrm{mM} \pm 0.3[215]\end{array}$ \\
\hline $\begin{array}{l}\text { TAS2R38- } \\
\text { PAV }\end{array}$ & $\begin{array}{l}\text { PROP (6- } n \text {-propylthio- } \\
\text { uracil) }\end{array}$ & $10-20 \mu \mathrm{M}$ & - & & $2.1 \mu \mathrm{M}[216]$ \\
\hline TAS2R43 & aristolochic acid & $62.5-500 \mathrm{nM}$ & $250-500 \mathrm{nM}$ & 4 & $\begin{array}{l}81 \mathrm{nM} \pm 0.0008 \\
{[217]}\end{array}$ \\
\hline \multirow[t]{3}{*}{ TAS2R46 } & picrotoxinin & $62.5-250 \mu \mathrm{M}$ & - & & $70 \mu \mathrm{M} \pm 5.2[218]$ \\
\hline & chloramphenicol & $125-500 \mu \mathrm{M}$ & - & & n.d. \\
\hline & denatonium benzoate & $62.5-125 \mu \mathrm{M}$ & $250-500 \mu \mathrm{M}$ & 4 & $240 \mu \mathrm{M} \pm 192[218]$ \\
\hline
\end{tabular}

*Concentration range in which an increasing response trend is observed n.d. EC50 not determined, but activation published in [33]
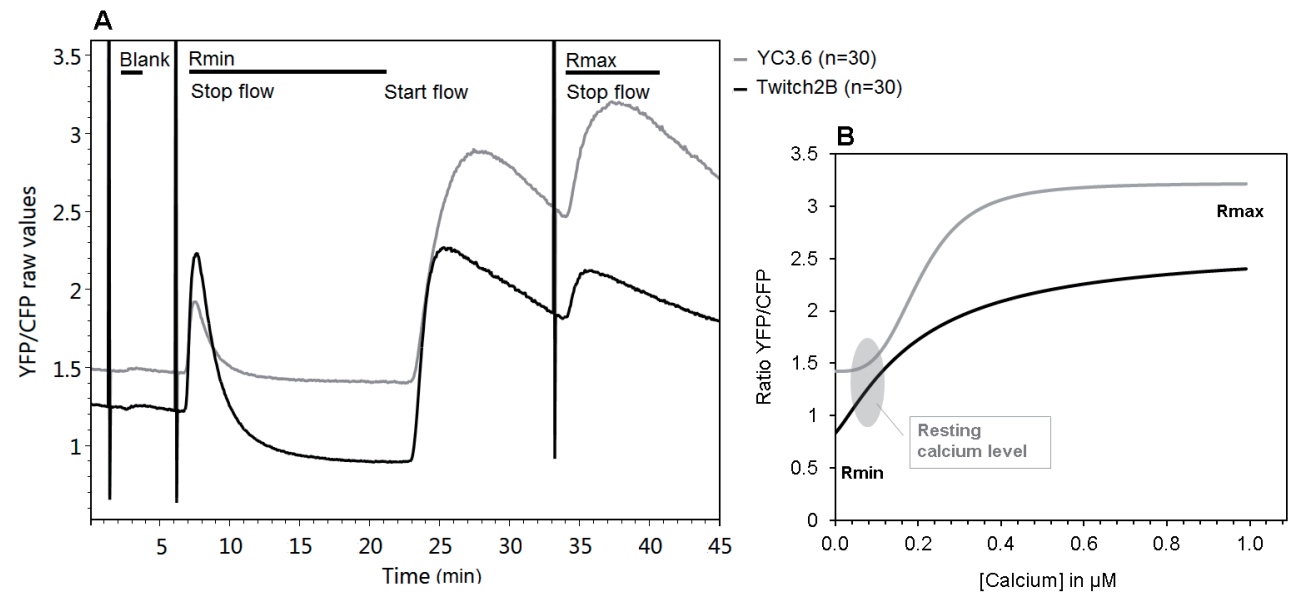

Figure 5, (A) Raw traces YFP FRET / CFP from spots expressing either Twitch2B or YC3.6. The flow was set at $200 \mu \mathrm{l} / \mathrm{min} \mathrm{C} 1$ buffer. After a blank injection, the $\mathrm{R}_{\min }$ buffer ( $\mathrm{C} 1$ buffer without $\mathrm{CaCl}_{2}$ but supplemented with $3 \mathrm{mM}$ EGTA and $5 \mu \mathrm{M}$ ionomycin) was injected. The flow was stopped at full $R_{\min }$ exposure and incubated for 15 minutes. Next the flow was started again replacing the $R_{\text {min }}$ buffer with the $\mathrm{C} 1$ buffer. Approx. 10 minutes later the $\mathrm{R}_{\max }$ buffer ( $\mathrm{C} 1$ buffer with $5 \mathrm{mM} \mathrm{CaCl}_{2}$ and $5 \mu \mathrm{M}$ lonomycin) was injected and the flow was stopped at full $R_{\text {max }}$ exposure. (B), Calculated theoretical calcium binding curves for YC3.6 and Twitch2B based on $R_{\min }$ and $R_{\max }$ measurements, the known dissociation constants for calcium and the Hill equation (See materials and methods section). 
calcium stores. When the flow was started again and the $\mathrm{R}_{\min }$ buffer was replaced by $\mathrm{C} 1$ buffer containing $2 \mathrm{mM} \mathrm{CaCl}_{2}$, there was a large increase in the ratio signal of both sensors because of calcium re-entering the cells. Twitch2B reached an $R_{\max }$ ratio of 2.6 and YC3.6 an $\mathrm{R}_{\max }$ of 3.2 when exposed to the $\mathrm{R}_{\max }$ buffer containing $5 \mathrm{mM} \mathrm{CaCl}_{2}$ and $5 \mu \mathrm{M}$ ionomycin. The dynamic range between $R_{\text {min }}$ and $R_{\text {max }}$ is quite comparable for both sensors, however, only Twitch2B clearly has an $R_{\text {min }}$ well below the cells' resting level (around $100 \mathrm{nM}$, [206]). Only Twitch2B can therefore be used to record very small changes in calcium ion concentration both above and below the resting level. Also the Hill curves based on the respective Hill coefficients for Twitch2B and YC3.6 (Figure 5B) confirm this.

\section{Discussion}

In this study we have evaluated the effectiveness of different genetically encoded calcium sensors, at a range of concentrations, for their ability to measure the comparatively low responses of bitter receptors. We observed strong response differences (4-5 fold) depending on sensor type and found that sensor Twitch2B yielded the strongest and most sensitive responses for eight of the evaluated bitter receptors. When compared to YC3.6, sensor Nano140 also yielded an improved calcium sensing, but Twitch2B appeared the best choice for bitter taste receptor arrays. In addition, we found a remarkably strong effect of the calcium sensor concentration on the sensitivity of the response. Lowering the gene dose 4 fold to $8 \mathrm{ng} / \mu \mathrm{l}$ resulted in about 2-3 fold lower protein concentrations [100], but improved the sensitivity for all sensors $4-15$ fold. Doses lower than $8 \mathrm{ng} / \mu \mathrm{l}$ sensor DNA in the print mix yielded arrays with poorly detectable baseline fluorescence (data not shown), and therefore $8 \mathrm{ng} / \mu \mathrm{l}$ sensor DNA was determined as optimal in our setup. The observed gene dose effects are a clear example of calcium buffering in our reverse transfected cell arrays. Endogenous calcium sensors, naturally present in cells, regulate calcium levels or transduce signals in response to changes in calcium concentration [219, 220]. These endogenous sensors bind and buffer part of the calcium which enters the cytosol. The remaining free calcium ions are available to the fluorescent calcium sensor. This calcium sensor also binds and buffers a fraction of the free calcium. This buffering by fluorescent calcium sensors is problematic when the calcium binding or buffering capacity of the sensor is in the same range or exceeding the calcium buffering capacity of the cell itself [221]. An effective way of reducing the contribution of this buffering effect and thereby measuring true calcium responses, is to lower the fluorescent calcium sensor concentration as much as possible [221]. Unfortunately, fluorescence based calcium sensors cannot be diluted too much due to fluorescence noise levels and limitations in detector sensitivity. As argued by McMahon et al. this means that for most genetically encoded and synthetic calcium fluorophores, the observed calcium dynamics are quenched even under our more optimal low probe conditions [221]. A future solution would be to switch to bioluminescence-based 
detection platforms for the measurement of cell calcium ion levels [222]. Photodetectors are generally regarded as 10-1000 times more sensitive than fluorescence detectors [223], allowing a further reduction in the amount of sensor needed for calcium detection and minimizing the calcium buffering.

Using optimal sensor concentrations, the two sensor types Twitch2B and YC3.6 were compared on sensitivity performance for a range of bitter taste receptors. The sensitivity was benchmarked against the EC50 values obtained from the literature studies employing calcium dyes, for each receptor-agonist pair. Except for the combinations TAS2R14/TAS2R46 with picrotoxinin and TAS2R38PAV with PROP, the published EC50 values are comparable to the response range obtained with receptor-Twitch2B combinations. The TAS2R46 response range to denatonium benzoate was lower than published. Deviations from the published values may relate to SNP variant differences (Suppl. Table 1) or to differences in the quality of the ligand stock solutions. Also the sensitivity of our specific imaging set up (filter, lens and camera) may play a role in missing very small effects. We rule out that it relates to lack of sensitivity of the Twitch2B probe because we show it is capable of following the modulation (lowering) of the resting concentration of cytosolic calcium (Figure 4).

The observed effects of the sensor type for sensors with similar dissociation constants can be explained by differences in the cooperative binding of calcium. Sensors with a Hill coefficient $>1$ have smaller ratio changes at calcium levels below their $K_{d}$ because of the sigmoidal shape of the calcium binding curve. We have confirmed this by comparing the calcium affinity curves of Twitch2B and YC3.6. The $R_{\min }$ of Twitch2B was found to be well below the resting calcium level of the HEK293 cells, while the YC3.6 sensor did not show a clear decrease in $\mathrm{R}_{\min }$ after chelating all calcium ions. With a sensitivity range that includes the resting values, the Twitch2B sensor is sensitive to minor changes in calcium. This makes it well suited for relatively weak responses to receptor agonists or inverse agonists as in case of bitter receptors. Furthermore, it has a more linear ratio increase so that it is less biased for a certain concentration range.

Twitch2B may be less suitable for detecting calcium dynamics above $300 \mathrm{nM}$ (as observed for some hormone receptors or ion channels) because, in that range, the sensor will become saturated (Figure 5 and [205]). YC3.6 may not be a good alternative for such receptors either, but other probes might be considered with a $K_{d}$ in the range of $>400 \mathrm{nM}$ $[91,224,225]$. Twitch2B may also be less suitable for application in ion-channel arrays since "leaky" calcium ion-channels can increase the resting levels of calcium within the cells [226]. Consequently, depending on the expected calcium signalling range, a wellconsidered choice of calcium sensor type has the potential to improve the sensitivity of the measurements although the linearity of the response from the resting level up (or down) is always an issue that should be taken into account. 


\section{Conclusion}

Overall, we conclude that by choosing sensor Twitch2B in a bitter receptor array, we could enhance the sensitivity of bitter taste receptor arrays up to 60 -fold compared to the more standard YC3.6. The response height increased 5-10 fold when the sensor concentration was also optimized. The receptor cell array contained 300 spots and could be exposed to a series of 18 injections within one hour. A triplicate experiment lasting three hours represents a high throughput analysis comprising 16,200 measurements and thus makes receptomics using flow cells a viable alternative to microtiter plate platforms. These optimised sensor conditions, together with a flow-through microfluidics format will benefit future receptomics experiments involving receptor cell arrays.

\section{Conflict of interests}

All authors declare no conflicts of interest. The founding sponsors had no role in the design of the study; in the collection, analyses, or interpretation of data; in the writing of the manuscript, and in the decision to publish the results.

\section{Funding}

This work was supported by the Dutch Topsector Horticulture and Propagation Materials, project "HTP phenotyping tomato flavour" KV 1409-025

\section{Acknowledgements}

Imaging facilities were offered by the Cell Biology and Immunology Group of Animal Sciences at WUR. Data analysis and visualization software was written by Marco van Lenthe from BU Biometris, Wageningen University and Research.

\section{Author contributions}

Conceptualization: MR, MAJ

Data Curation: MR

Data Analysis: MR, RW

Funding Acquisition: MAJ

Gene cloning: MGLH, MR

Writing \{ Original Draft Preparation: MR, RW, MAJ

Writing \{ Review \& Editing: MR, MAJ, RW, MGLH, RDH, RFW 


\section{Supplemental Figures and Tables}

\section{Array 1: R8}

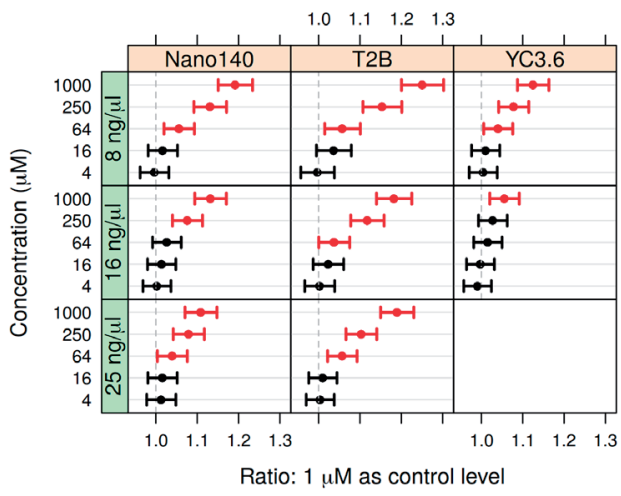

Array 2: R8

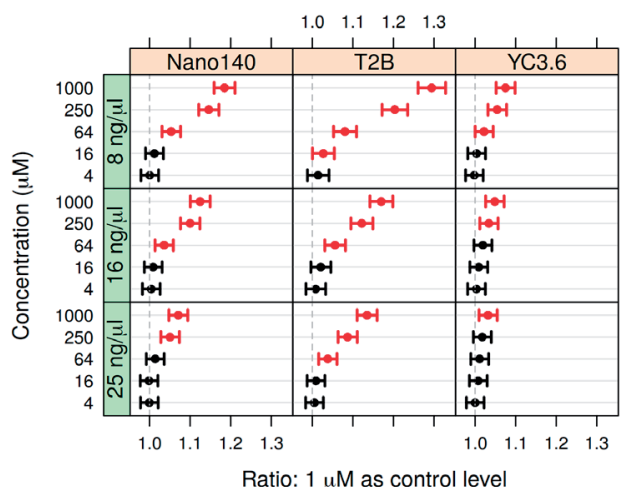

Array 1: R14

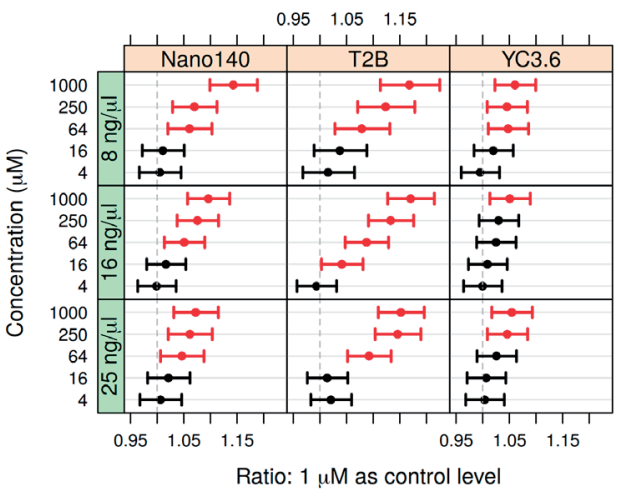

Array 2: R14

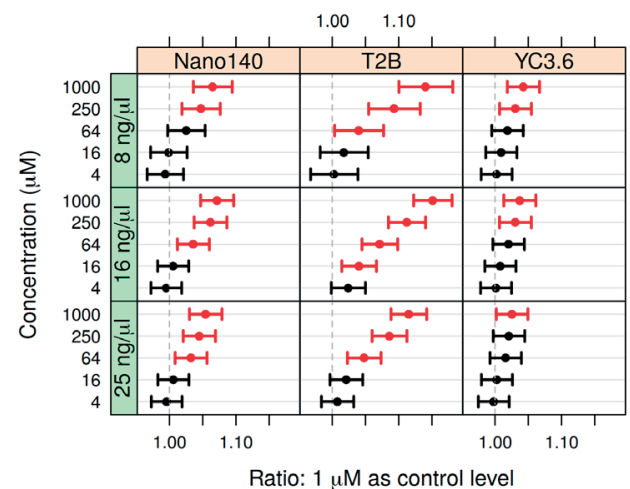

Supplemental Figure 1, Response values of TAS2R8 and TAS2R14 for stimulation with agonist mixture (chloramphenicol and picrotoxinin) for both arrays at sensor gene dose of 8, 16 and $25 \mathrm{ng} /$ $\mu \mathrm{l}$. Error bars present the $95 \%$ confidence interval of the response value. Response values represent estimates of treatment-versus-control contrasts with respect to the $1 \mu \mathrm{M}$ injection. T2B, Twitch2B. 


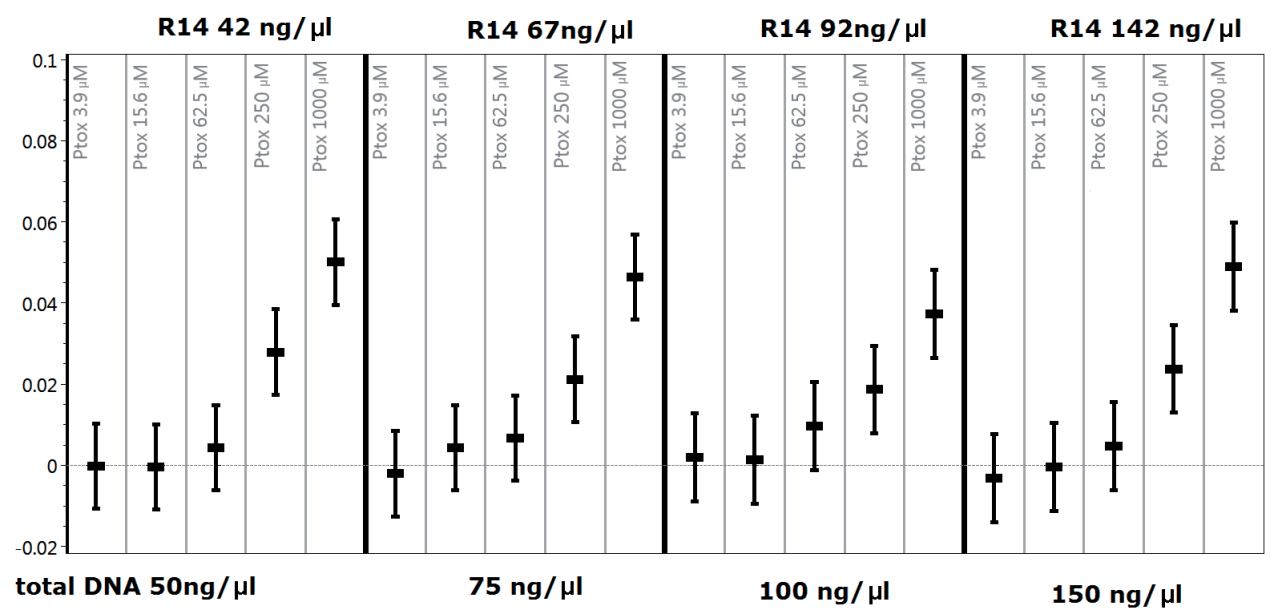

Supplemental Figure 2, Relation between total DNA and receptor (TAS2R14) coding DNA in the print mix. The calcium sensor Twitch2B was added to the mix at $8 \mathrm{ng} / \mu \mathrm{l}$. PTox, picrotoxinin. 
Series 1: D-Sal

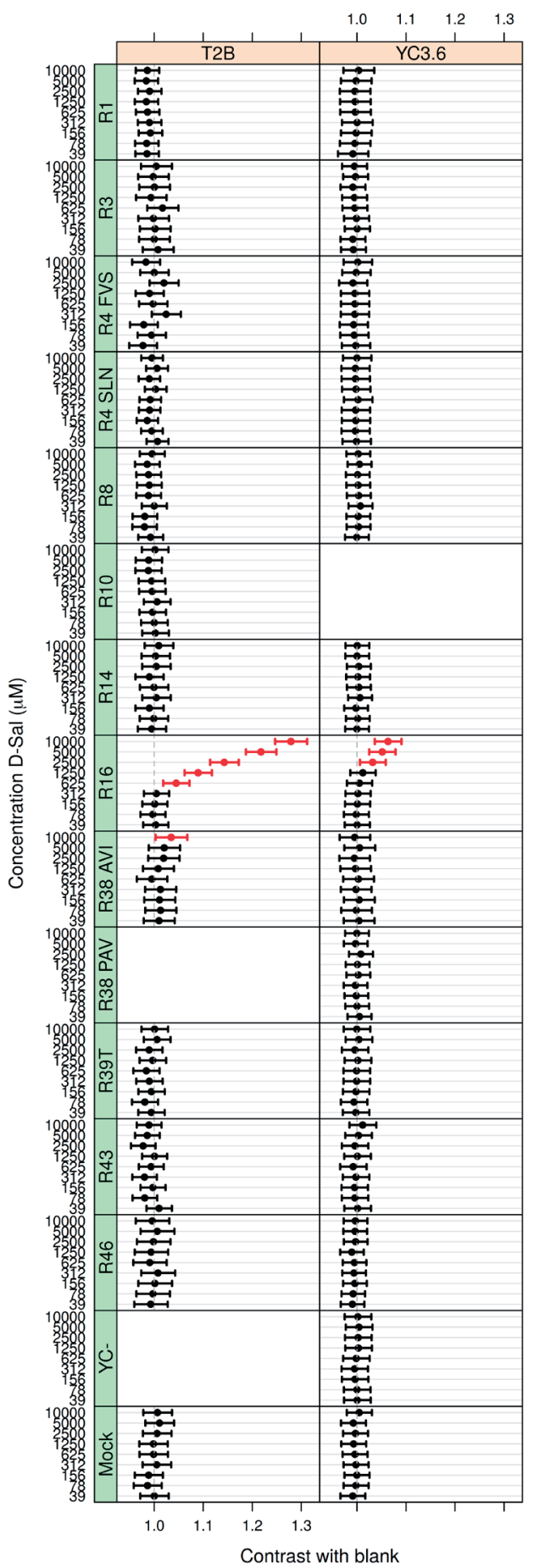

Series 1: PTox

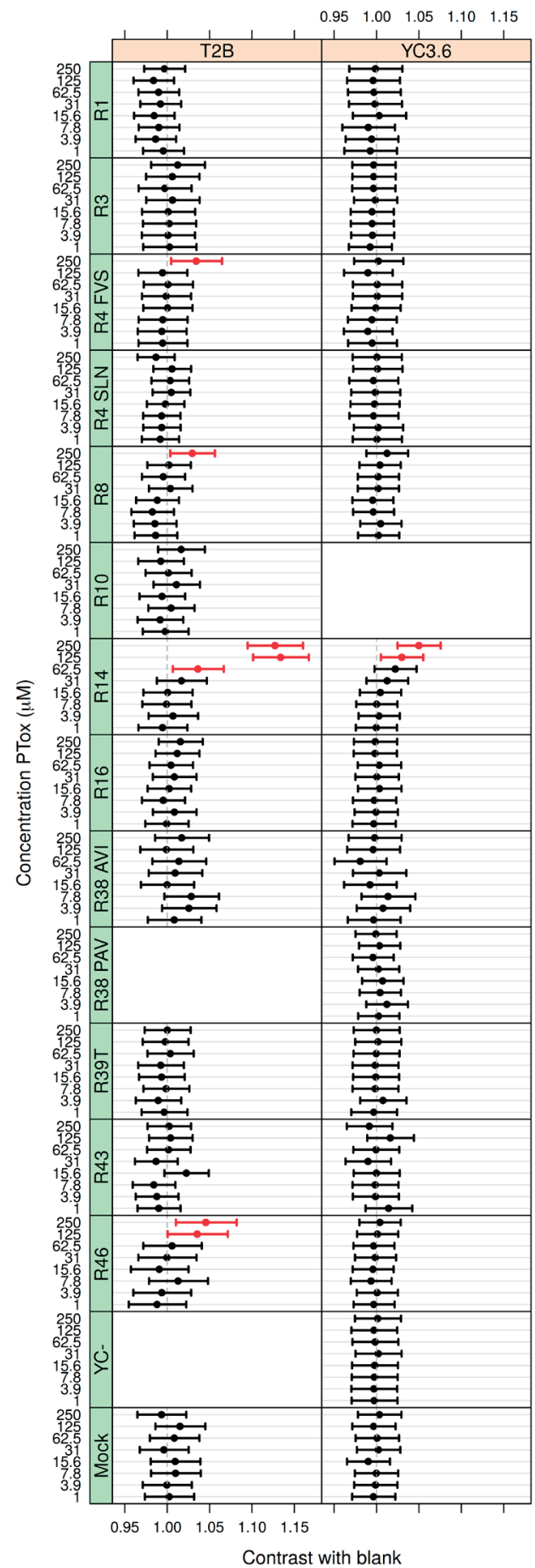


Series 2: CHL

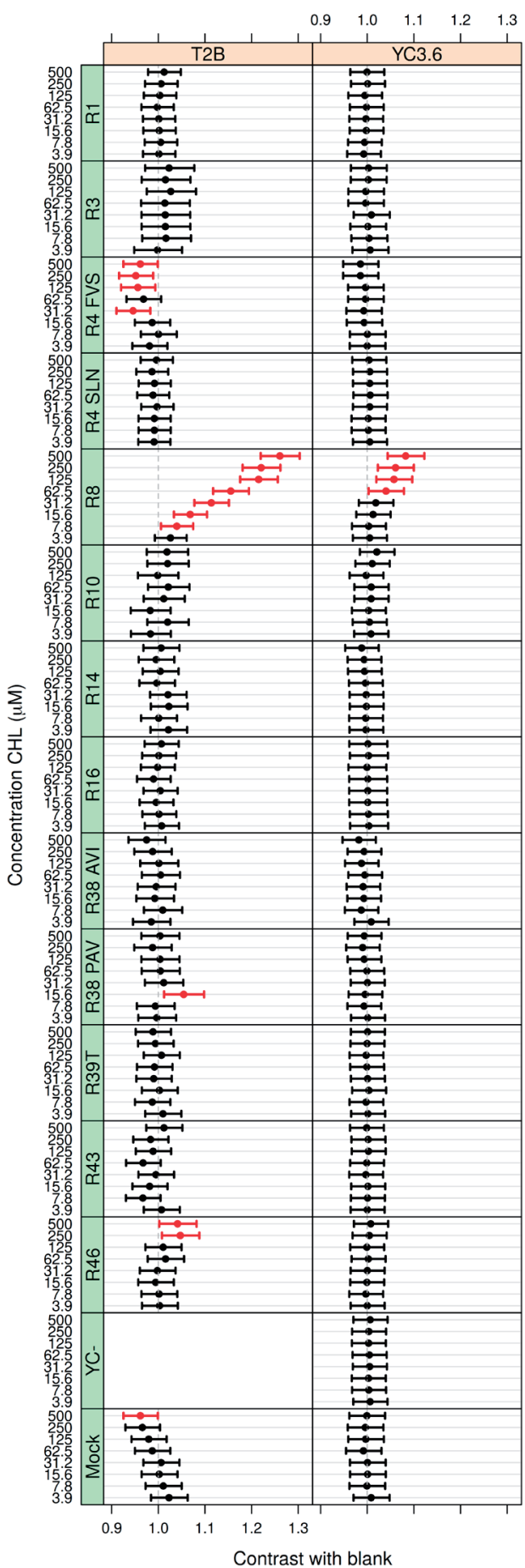

Series 2: PROP

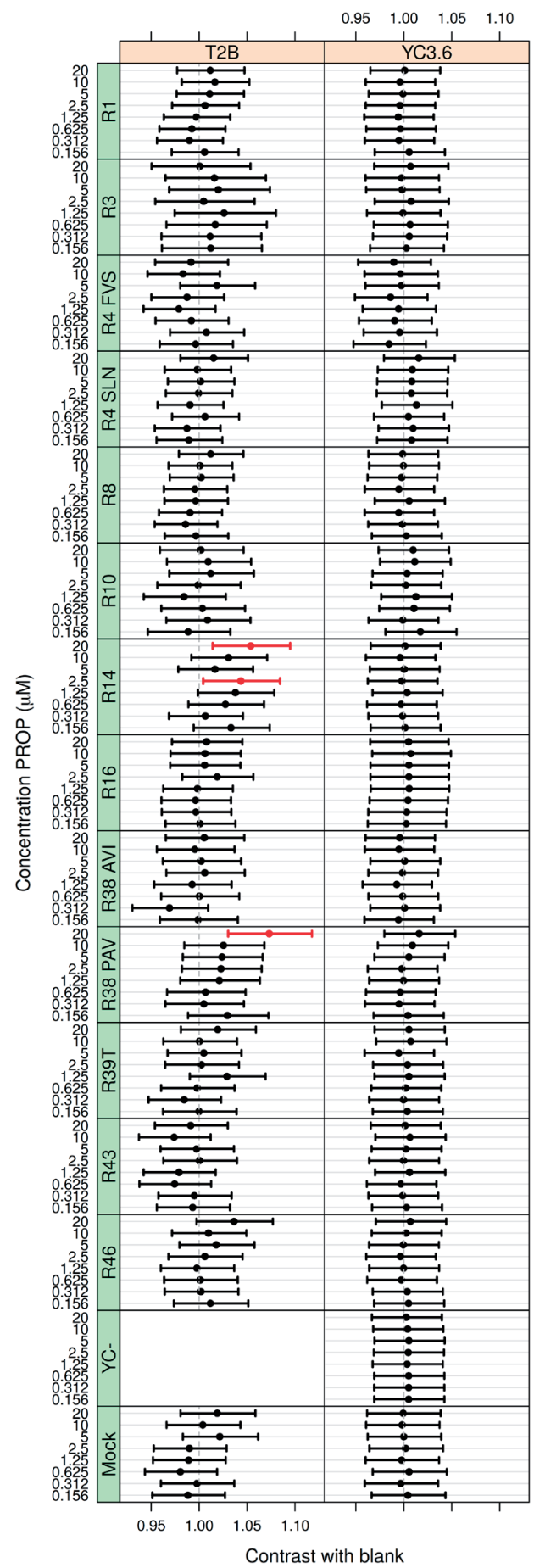


Series 3: AA

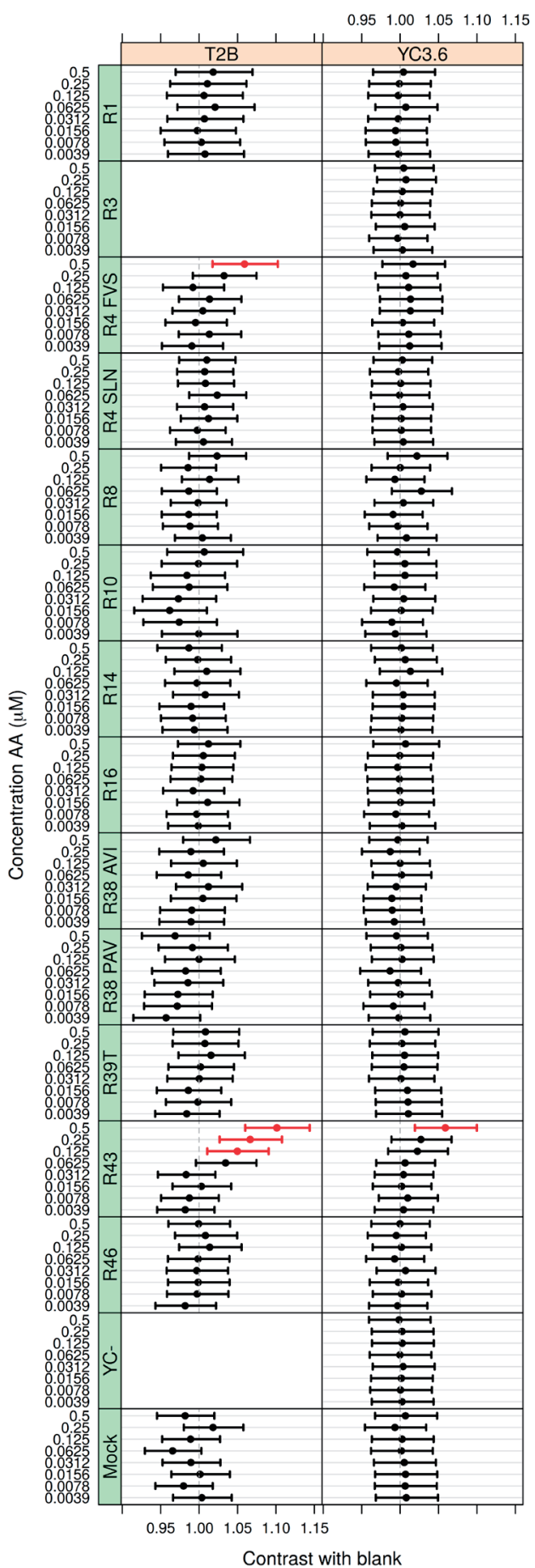

Series 3: DB

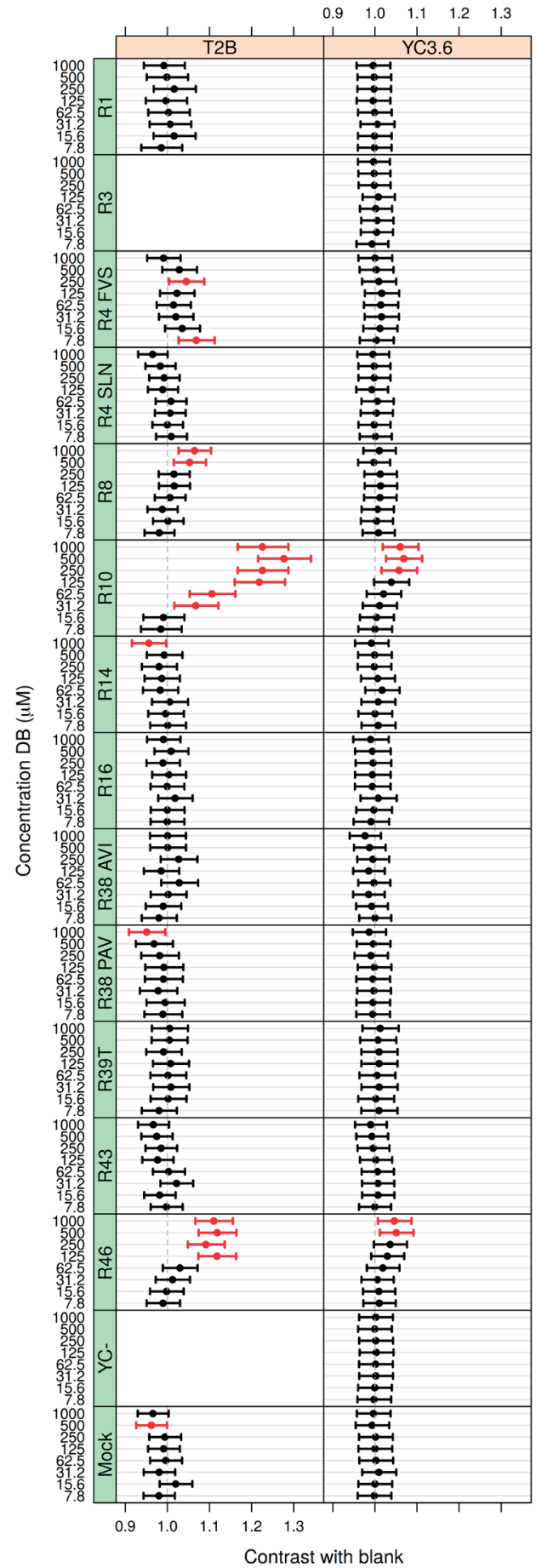



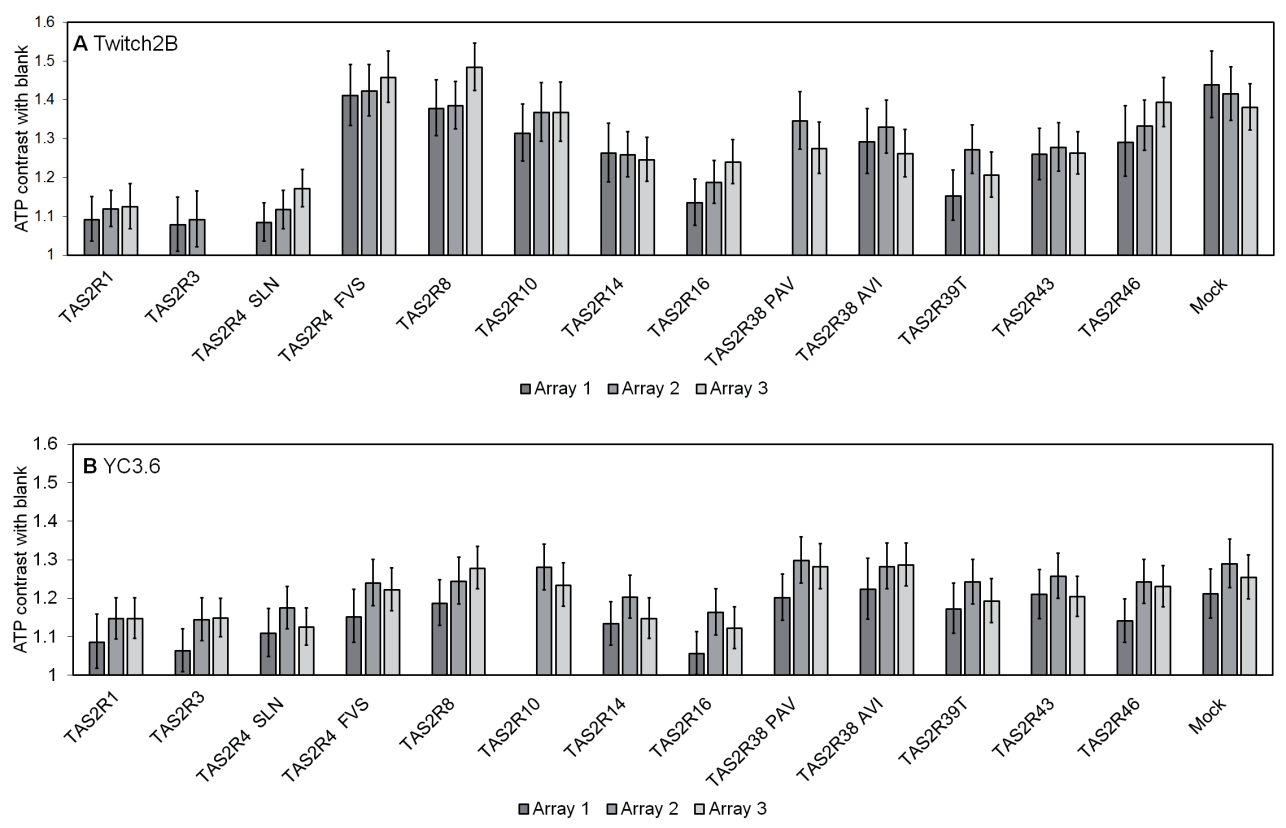

Supplemental Figure 4, Metabolic exhaustion measurement by ATP exposure at the end of each injection series. ATP responses were compared to the blank for all bitter receptor spot types combined with either Twitch2B (A) or YC3.6 (B) for the three arrays. Array 1 was exposed to $10 \mu \mathrm{M}$ ATP for and Array 2 and 3 to $5 \mu \mathrm{M}$ ATP. Array 1 featured alternating injections of D-salicin and picrotoxinin which activated TAS2R16 and TAS2R14/TAS2R46. Array 2 featured alternating injections of chloramphenicol and PROP which activated TAS2R8/TAS2R46 and TAS2R38PAV. Array 3 featured alternating injections of Aristocholic acid and denatonium benzoate which activated TAS2R43 and TAS2R8/TAS2R10/TAS2R46. Error bars show a 95\% confidence interval for the response estimates.

\footnotetext{
4 Supplemental Figure 3, Response values of TAS2R and mock spots stimulated with alternating injected samples. Each series is measured on a separate array. Error bars present the $95 \%$ confidence interval of the response value. Response values represent estimates of treatment-versus-control contrasts with respect to the blank injection. Sets with less than 5 spots were excluded. YC- control for optical changes (air bubbles, auto-fluorescence etc.) is a deletion mutant of YC3.6 ( $\triangle$ A332-S392) with fixed FRET between CFP and YFP. T2B, Twitch2B, AA, aristolochic acid, CHL, chloramphenicol, PTox, picrotoxinin, D-Sal, D-salicin, DB, denatonium benzoate and PROP, 6-n-propylthiouracil.
} 
Supplemental Table 1, Bitter taste receptor genes

\begin{tabular}{|c|c|c|c|c|c|}
\hline $\begin{array}{l}\text { Bitter } \\
\text { receptor }\end{array}$ & $\begin{array}{l}\text { Alter- } \\
\text { native } \\
\text { name }\end{array}$ & $\begin{array}{l}\text { Uniprot } \\
\text { listed } \\
\text { natural } \\
\text { variants }\end{array}$ & $\begin{array}{l}\text { Uniprot } \\
\text { code* }\end{array}$ & $\begin{array}{l}\text { Variant } \\
\text { used } \\
\text { in this } \\
\text { study }\end{array}$ & $\begin{array}{l}\text { Amino acid sequence used in this study } \\
\text { with variant positions highlighted** }\end{array}$ \\
\hline TAS2R1 & TRB7 & $\begin{array}{l}\text { R111H, } \\
\text { C141Y, } \\
\text { R206W }\end{array}$ & Q9NYW7 & $\begin{array}{l}111 R, \\
141 C, \\
206 R\end{array}$ & $\begin{array}{l}\text { MLESHLIIYFLLAVIQFLLGIFTNGIIV } \\
\text { VVNGIDLIKHRKMAPLDLLLSCLAVS } \\
\text { RIFLQLFIFYVNVIVIFFIEFIMC } \\
\text { SANCAILLFINELELWLATWLGVFY } \\
\text { CAKVASVRHPLFIWLKMRISKLVPWMILG } \\
\text { SLLYVSMICVFHSKYAGFMVPYFLRK } \\
\text { FFSQNATIQKEDTLAIQIFSFVAEFSVPL } \\
\text { LIFLFAVLLLIFSLGRHTRQMRNTVAGS } \\
\text { RVPGRGAPISALLSILSFLILYFSHC } \\
\text { MIKVFLSSLKFHIRRFIFLFFILVIGIYPS } \\
\text { GHSLILILGNPKLKQNAKKFLLHSKCCQ }\end{array}$ \\
\hline TAS2R3 & & & Q9NYW6 & & $\begin{array}{l}\text { MMGLTEGVFLILSGTQFTLGIL } \\
\text { VNCFIELVNGSSWFKTKRMSLSDFIIT } \\
\text { TLALLRIILLCIILTDSFLIEFSPNTHDS } \\
\text { GIIMQIIDVSWTFTNHLSIWLATCLGVL } \\
\text { YCLKIASFSHPTFLWLKWRVSRVMVWM } \\
\text { LLGALLLSCGSTASLINEFKLYSVFR } \\
\text { GIEATRNVTEHFRKKRSEYYLIHVLGTL } \\
\text { WYLPPLIVSLASYSLLIFSLGRHTRQM } \\
\text { LQNGTSSRDPTTEAHKRAIRIILSFFFLFL } \\
\text { LYFLAFLIASFGNFLPKTKMAKMIGEVMT } \\
\text { MFYPAGHSFILILGNSKLKQTFVVMLRCES } \\
\text { GHLKPGSKGPIFS }\end{array}$ \\
\hline TAS2R4 & & $\begin{array}{l}\text { R3Q, F7S, } \\
\text { F62L, } \\
\text { T74M, } \\
\text { V96L, } \\
\text { S171N, } \\
\text { I191V }\end{array}$ & Q9NYW5 & $\begin{array}{l}\text { (SLN) 3R, } \\
7 \mathrm{~S}, 62 \mathrm{~F}, \\
74 T, 96 \mathrm{~L}, \\
171 \mathrm{~N}, \\
191 \mathrm{l} \text { and }\end{array}$ & $\begin{array}{l}\text { MLRLFYSSAIIASVILNEVGIIMNLFITV } \\
\text { VNCKTWVKSHRISSSDRILFSLGITRELM } \\
\text { LGLFLVNTIYFVSSNTERSVYLSAFFV } \\
\text { LCFMFLDSSSLWFVTLLNILYCVKITN } \\
\text { FQHSVFLLLKRNISPKIPRLLLACVLIS } \\
\text { AFTTCLYITLSQASPFPELVTTRNNTSFNI } \\
\text { NEGILSLVVSLVLSSSLQFIINVTSASLLI } \\
\text { HSLRRHIQKMQKNATGFWNPQTEAHVGAM } \\
\text { KLMVYFLILYIPYSVATLVQYLPFYAGMD } \\
\text { MGTKSICLIFATLYSPGHSVLIIITHPKLK } \\
\text { TTAKKILCFKN }\end{array}$ \\
\hline TAS2R4 & & $\begin{array}{l}\text { R3Q, F7S, } \\
\text { F62L, } \\
\text { T74M, } \\
\text { V96L, } \\
\text { S171N, } \\
\text { I191V }\end{array}$ & Q9NYW5 & $\begin{array}{l}\text { (FVS) 3R, } \\
7 \mathrm{~F}, 62 \mathrm{~F}, \\
74 \mathrm{~T}, 96 \mathrm{~V}, \\
171 \mathrm{~S}, \\
191 \mathrm{I}\end{array}$ & $\begin{array}{l}\text { MLRLFYFSAIIASVILNFVGIIMNLFITV } \\
\text { VNCKTWVKSHRISSSDRILFSLGITRELM } \\
\text { LGLFLVNTIYFVSSNTERSVYLSAFEV } \\
\text { LCFMFLDSSSVWFVTLLNILYCVKITN } \\
\text { FQHSVFLLLKRNISPKIPRLLLACVLIS } \\
\text { AFTTCLYITLSQASPFPELVTTRNNTSF } \\
\text { NISEGILSLVVSLVLSSSLQFIINVTSASL } \\
\text { LIHSLRRHIQKMQKNATGFWNPQTEAH } \\
\text { VGAMKLMVYFLILYIPYSVATLVQYLP } \\
\text { FYAGMDMGTKSICLIFATLYSPGHSVLII } \\
\text { ITHPKLKTTAKKILCFKN }\end{array}$ \\
\hline
\end{tabular}


Supplemental Table 1, Continued

\begin{tabular}{|c|c|c|c|c|c|}
\hline $\begin{array}{l}\text { Bitter } \\
\text { receptor }\end{array}$ & $\begin{array}{l}\text { Alter- } \\
\text { native } \\
\text { name }\end{array}$ & $\begin{array}{l}\text { Uniprot } \\
\text { listed } \\
\text { natural } \\
\text { variants }\end{array}$ & $\begin{array}{l}\text { Uniprot } \\
\text { code* }\end{array}$ & $\begin{array}{l}\text { Variant } \\
\text { used } \\
\text { in this } \\
\text { study }\end{array}$ & $\begin{array}{l}\text { Amino acid sequence used in this study } \\
\text { with variant positions highlighted** }\end{array}$ \\
\hline TAS2R8 & TRB5 & M308V & Q9NYW2 & $308 \mathrm{M}$ & $\begin{array}{l}\text { MFSPADNIFIILITGEFILGILGNGY } \\
\text { IALVNWIDWIKKKKISTVDYILTNLVIARI } \\
\text { CLISVMVVNGIVIVLNPDVYTKNKQQIV } \\
\text { IFTFWTFANYLNMWITTCLNVFYFLKI } \\
\text { ASSSHPLFLWLKWKIDMVVHWILLG } \\
\text { CFAISLLVSLIAAIVLSCDYRFHAIAKH } \\
\text { KRNITEMFHVSKIPYFEPLTLFNLFAIVP } \\
\text { FIVSLISFFLLVRSLWRHTKQIK } \\
\text { LYATGSRDPSTEVHVRAIKTMTSFIFFFF } \\
\text { LYYISSILMTFSYLMTKYKLAVEFGEIAAI } \\
\text { LYPLGHSLILIVLNNKLRQTFVRMLTCRK } \\
\text { IACMI }\end{array}$ \\
\hline TAS2R10 & TRB2 & M156T & Q9NYW0 & $156 \mathrm{M}$ & $\begin{array}{l}\text { MLRVVEGIFIFVVVSESVFGVLGNG } \\
\text { FIGLVNCIDCAKNKLSTIGFILTGLAIS } \\
\text { RIFLIWIIITDGFIQIFSPNIYASGNLIEY } \\
\text { ISYFWVIGNQSSMWFATSLSIFYFLKIAN } \\
\text { FSNYIFLWLKSRTNMVLPFMIVFLLISSLL } \\
\text { NFAYIAKILNDYKMKNDTVWDLNMYKSEY } \\
\text { FIKQILLNLGVIFFFTLSLITCIFLIIS } \\
\text { LWRHNRQMQSNVTGLRDSNTEAHVKAM } \\
\text { KVLISFIILFILYFIGMAIEISCFTVREN } \\
\text { KLLLMFGMTTTAIYPWGHSFILILGNSK } \\
\text { LKQASLRVLQQLKCCEKRKNLRVT } \\
\end{array}$ \\
\hline TAS2R14 & TRB1 & $\begin{array}{l}\text { T86A, } \\
\text { L201F }\end{array}$ & Q9NYV8 & $\begin{array}{l}\text { 86T, } \\
\text { 201L, } \\
\text { R299K }\end{array}$ & $\begin{array}{l}\text { MGGVIKSIFTFVLIVEFIIGNLGNS } \\
\text { FIALVNCIDWVKGRKISSVDRILTALAIS } \\
\text { RISLVWLIFGSWCVSVFFPALFATEKM } \\
\text { FRMLTNIWTVINHFSVWLATGLGTFY } \\
\text { FLKIANFSNSIFLYLKWRVKKVVLV } \\
\text { LLLVTSVFLFLNIALINIHINASINGYRRN } \\
\text { KTCSSDSSNFTRFSSLIVLTSTVFIFIP } \\
\text { FTLSLAMFLLLIFSMWKHRKKMQHTVKIS } \\
\text { GDASTKAHRGVKSVITFFLLYAIFSLS } \\
\text { FFISVWTSERLEENLIILSQVMGMAYP } \\
\text { SCHSCVLILGNKKLRQASLSVLLWLKYM } \\
\text { FKDGEPSGHKEFRESS }\end{array}$ \\
\hline TAS2R16 & & $\begin{array}{l}\text { V101M, } \\
\text { I114V, } \\
\text { L116P, } \\
\text { P161S, } \\
\text { N172K, } \\
\text { Q177P, } \\
\text { N216D, } \\
\text { A221V, } \\
\text { R222H, } \\
\text { V235M, } \\
\text { F240V }\end{array}$ & Q9NYV7 & $\begin{array}{l}101 \mathrm{~V} \\
114 \mathrm{I} \\
116 \mathrm{~L} \\
161 \mathrm{P}, \\
172 \mathrm{~N}, \\
177 \mathrm{Q} \\
216 \mathrm{~N} \\
221 \mathrm{~A} \\
222 \mathrm{H} \\
235 \mathrm{~V} \\
240 \mathrm{~F}\end{array}$ & $\begin{array}{l}\text { MIPIQLTVFFMIIYVLESLTIIVQSSLIV } \\
\text { AVLGREWLQVRRLMPVDMILISLGIS } \\
\text { RFCLQWASMLNNFCSYFNLNYVLCNL } \\
\text { TITWEFFNILTFWLNSLLTVFYCIKVSS } \\
\text { FTHHIFLWLRWRILRLFPWILLGSL } \\
\text { MITCVTIIPSAIGNYIQIQLLTMEHLPRN } \\
\text { STVTDKLENFHQYQFQAHTVALVIPFIL } \\
\text { FLASTIFLMASLTKQIQHHSTGHCNPS } \\
\text { MKAHFTALRSLAVLFIVFTSYFLTIL } \\
\text { ITIIGTLFDKRCWLWVWEAFVYAFILM } \\
\text { HSTSLMLSSPTLKRILKGKC }\end{array}$ \\
\hline
\end{tabular}


Supplemental Table 1, Continued

\begin{tabular}{|c|c|c|c|c|c|}
\hline $\begin{array}{l}\text { Bitter } \\
\text { receptor }\end{array}$ & $\begin{array}{l}\text { Alter- } \\
\text { native } \\
\text { name }\end{array}$ & $\begin{array}{l}\text { Uniprot } \\
\text { listed } \\
\text { natural } \\
\text { variants }\end{array}$ & $\begin{array}{l}\text { Uniprot } \\
\text { code* }\end{array}$ & $\begin{array}{l}\text { Variant } \\
\text { used } \\
\text { in this } \\
\text { study }\end{array}$ & $\begin{array}{l}\text { Amino acid sequence used in this study } \\
\text { with variant positions highlighted** }\end{array}$ \\
\hline TAS2R38 & TAS2R61 & $\begin{array}{l}\text { A49P, } \\
\text { V262A, } \\
\text { I296V }\end{array}$ & P59533 & $\begin{array}{l}\text { (PAV)49P, } \\
262 A \\
296 V\end{array}$ & $\begin{array}{l}\text { MLTLTRIRTVSYEVRSTFLFISVLEFAVG } \\
\text { FLTNAFVFLVNFWDVVKRQPLSNSDCVLL } \\
\text { CLSISRLFLHGLLFLSAIQLTHFQKLSE } \\
\text { PLNHSYQAIIMLWMIANQANLWLAACLSL } \\
\text { LYCSKLIRFSHTFLICLASWVSRKISQM } \\
\text { LLGIILCSCICTVLCVWCFFSRPHFTVT } \\
\text { TVLFMNNNTRLNWQIKDLNLFYSFLF } \\
\text { CYLWSVPPFLLFLVSSGMLTVSLGRHMRT } \\
\text { MKVYTRNSRDPSLEAHIKALKSLVSFF } \\
\text { CFFVISSCAAFISVPLLILWRDKIGVM } \\
\text { VCVGIMAACPSGHAAVLISGNAKLRRAVM } \\
\text { TILLWAQSSLKVRADHKADSRTLC }\end{array}$ \\
\hline TAS2R38 & TAS2R61 & $\begin{array}{l}\text { A49P, } \\
\text { V262A, } \\
\text { I296V }\end{array}$ & P59533 & $\begin{array}{l}\text { (AVI) } \\
49 A, \\
262 V, \\
296 I\end{array}$ & $\begin{array}{l}\text { MLTLTRIRTVSYEVRSTFLFISVLEFAVG } \\
\text { FLTNAFVFLVNFWDVVKRQALSNSDCVLL } \\
\text { CLSISRLFLHGLLFLSAIQLTHFQKLSE } \\
\text { PLNHSYQAIIMLWMIANQANLWLAACLSL } \\
\text { LYCSKLIRFSHTFLICLASWVSRKISQM } \\
\text { LLGIILCSCICTVLCVWCFFSRPHFTVT } \\
\text { TVLFMNNNTRLNWQIKDLNLFYSFLF } \\
\text { CYLWSVPPFLLFLVSSGMLTVSLGRHMRT } \\
\text { MKVYTRNSRDPSLEAHIKALKSLVSFF } \\
\text { CFFVISSCVAFISVPLLILWRDKIGVM } \\
\text { VCVGIMAACPSGHAAILISGNAKLRRAVM } \\
\text { TILLWAQSSLKVRADHKADSRTLC }\end{array}$ \\
\hline TAS2R39 & TAS2R57 & $\begin{array}{l}\text { S193F, } \\
\text { K197E }\end{array}$ & P59534 & $\begin{array}{l}\text { 193S, } \\
\text { 197K, } \\
\text { A301T }\end{array}$ & $\begin{array}{l}\text { MLGRCFPPDTKEKQQLRMTKLCDPAE } \\
\text { SELSPFLITLILAVLLAEYLIGIIANGFI } \\
\text { MAIHAAEWVQNKAVSTSGRILVFLSVS } \\
\text { RIALQSLMMLEITISSTSLSFYSEDAVY } \\
\text { YAFKISFIFLNFCSLWFAAWLSFFYFVKI } \\
\text { ANESYPLFLKLRWRITGLIPWLLWLSVFIS } \\
\text { FSHSMFCINICTVYCNNSFPIHSSNSTKK } \\
\text { TYLSEINVVGLAFFFNLGIVTPLIMFIL } \\
\text { TATLLILSLKRHTLHMGSNATGSNDPS } \\
\text { MEAHMGAIKAISYFLILYIFNAVALFI } \\
\text { YLSNMFDINSLWNNLCQIIMTAYPASHSIL } \\
\text { LIQDNPGLRRAWKRLQLRLHLYPKEWTL }\end{array}$ \\
\hline TAS2R43 & TAS2R52 & & P59537 & $\begin{array}{l}35 S \\
212 R\end{array}$ & $\begin{array}{l}\text { MITFLPIIFSSLVVVTFVIGNFANGFI } \\
\text { ALVNSIESFKRQKISFADQILTALAVS } \\
\text { RVGLLWVLLLNWYSTVLNPAFNSVEVRT } \\
\text { TAYNIWAVINHFSNWLATTLSIFYLLKI } \\
\text { ANFSNFIFLHLKRRVKSVILVMLLGPLL } \\
\text { FLACHLFVINMNEIVRTKEFEGNMTWK } \\
\text { IKLKSAMYFSNMTVTMVANLVPFTLTLLS } \\
\text { FMLLICSLCKHLKKMQLRGKGSQDPST } \\
\text { KVHIKALQTVISFLLLCAIYFLSIMISVWS } \\
\text { FGSLENKPVFMFCKAIRFSYPSIHPFILIW } \\
\text { GNKKLKQTFLSVFWQMRYWVKGEKTSSP }\end{array}$ \\
\hline
\end{tabular}


Supplemental Table 1, Continued

\begin{tabular}{|c|c|c|c|c|c|}
\hline $\begin{array}{l}\text { Bitter } \\
\text { receptor }\end{array}$ & $\begin{array}{l}\text { Alter- } \\
\text { native } \\
\text { name }\end{array}$ & $\begin{array}{l}\text { Uniprot } \\
\text { listed } \\
\text { natural } \\
\text { variants }\end{array}$ & $\begin{array}{l}\text { Uniprot } \\
\text { code* }\end{array}$ & $\begin{array}{l}\text { Variant } \\
\text { used } \\
\text { in this } \\
\text { study }\end{array}$ & $\begin{array}{l}\text { Amino acid sequence used in this study } \\
\text { with variant positions highlighted** }\end{array}$ \\
\hline TAS2R46 & TAS2R54 & L228M & P59540 & $228 \mathrm{~L}$ & $\begin{array}{l}\text { MITFLPIIFSILIVVTFVIGNFANG } \\
\text { FIALVNSIEWEKRQKISFADQILTA } \\
\text { LAVSRVGLLWVLVLNWYATELNPAFN } \\
\text { SIEVRITAYNVWAVINHFSNWLATSLSI } \\
\text { FYLLKIANESNLIFLHLKRRVKSVVL } \\
\text { VILLGPLLFLVCHLFVINMNQIIWTKEYEG } \\
\text { NMTWKIKLRSAMYLSNTTVTILANLVP } \\
\text { FTLTLISFLLLICSLCKHLKKMQLHG } \\
\text { KGSQDPSMKVHIKALQTVTSFLLLCAI } \\
\text { YFLSIIMSVWSFESLENKPVFMFCEAIAF } \\
\text { SYPSTHPFILIWGNKKLKQTFLSVLWHVRY } \\
\text { WVKGEKPSSS }\end{array}$ \\
\hline
\end{tabular}

*https://www.uniprot.org

** variants include known and unpublished amino acid mutations (in bold) 

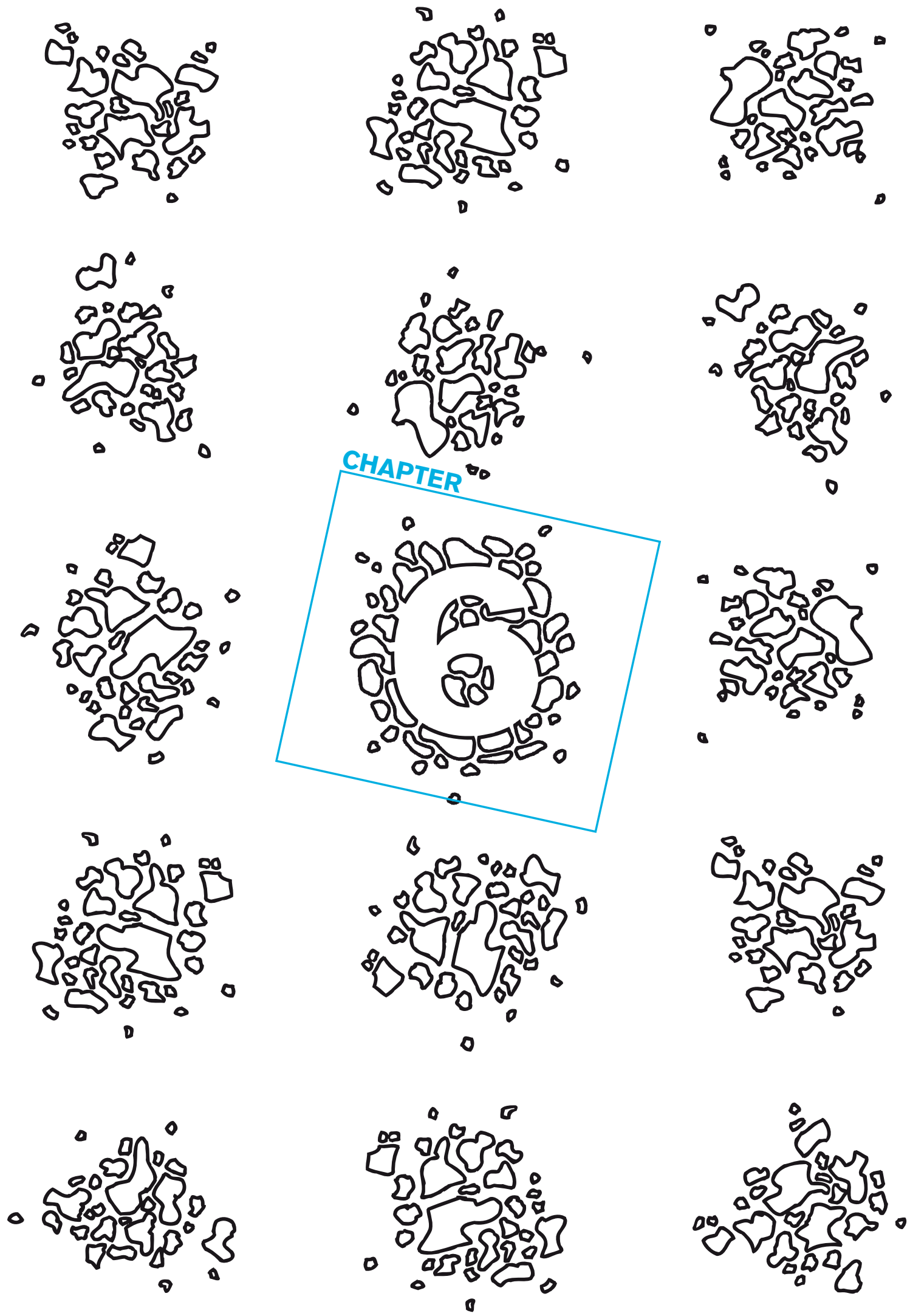


\section{Statistical models}

discriminating between complex samples measured with microfluidic receptor-cell arrays

Ron Wehrens, Margriet Roelse, Maurice G.L. Henquet, Marco van Lenthe, Paul W. Goedhart and Maarten A. Jongsma

R. Wehrens et al, PLoS ONE 2019, 14(4) 1-16

DOI: $10.1371 /$ journal.pone. 0214878 


\begin{abstract}
Data analysis for flow-based in-vitro receptomics array, like a tongue-on-a-chip, is complicated by the relatively large variability within and between arrays, transfected DNA types, spots, and cells within spots. Simply averaging responses of spots of the same type would lead to high variances and low statistical power. This paper presents an approach based on linear mixed models, allowing a quantitative and robust comparison of complex samples and indicating which receptors are responsible for any differences. These models are easily extended to take into account additional effects such as the build-up of cell stress and to combine data from replicated experiments. The increased analytical power this brings to receptomics research is discussed.
\end{abstract}




\section{Introduction}

Receptomics research with microfluidic receptor cell arrays aims to measure purely biological responses without a complicated biological system surrounding it [95, 227, 228]. For example, the human tongue can be emulated on a chip by an array containing G-protein coupled receptors (GPCRs), e.g., in the form of reconstituted receptor proteins [229] or vesicles [230]. Another possibility is formed by living cells expressing the genes coding for particular GPCRs, produced by either reverse-transfecting a generic cell line on the chip [100] or spotting pre-transfected cells [94]. Such a tongue-on-a-chip allows direct access to the original taste signal, before the signal is further transmitted via neurons, and processed and interpreted by the human brain. Thus, while a taste panellist would define a sample as bitter or sweet, a tongue-on-a-chip provides direct quantitative information on which taste receptors are triggered, and by how much. On a more general level, receptomics enables identifying compounds or extracts activating or blocking specific receptors active in taste sensation as well as in many other processes. Humans have a wide palette of receptor proteins. Even considering only GPCRs there are more than 800 receptors for the detection of hormones, neurotransmitters, tastants, odorants, and others. Since all receptors play an important role in human physiology, there is an advantage to a receptomics approach aiming at combining different receptors on a single chip, allowing the researcher to study the role of a compound or extract in a wider perspective by including all or at least the most relevant receptors.

However, data analysis for flow-based in-vitro biosensor arrays such as a tongue-on-a-chip is complicated by the relatively large variability in specific and non-specific responses within and between arrays caused by differences in the expression of the transfected receptor DNA, variability in spots and in cells within spots. Approaches based on simply averaging the response values of spots of the same type therefore lead to estimates with a large variability, allowing only the most obvious differences between samples to be detected and providing no means to correct for other effects often seen in flow-cell based approaches such as time or memory effects. Furthermore, chemically complex samples may trigger host-cell responses that vary widely between receptor types, depending on unknown interactions with the functional properties of the transfected DNA [231]. To eliminate such host-cell responses often hampering the analysis, one may dilute the sample until the hostcell response is no longer observed, but that often also means losing a large part or even all of the signal.

Here, we are focusing on microfluidic receptomics chips containing receptor cell arrays generated by reverse transfection of DNA arrays and with ectopic expression of different GPCRs and a generic calcium-ion sensor protein, Twitch2B [100, 205]. The chips were printed with plasmid DNA encoding a GPCR gene and a calcium sensor gene. Transient expression 
of the genes was achieved by reverse transfection: HEK293 cell were seeded on top of the DNA array, the DNA was taken up and the genes were expressed leading to G-proteincoupled receptors embedded in the cell membrane and calcium sensor accumulation in the cytoplasm. Upon stimulation of the receptor with a ligand, the GPCR signal transduction pathway is activated via $\mathrm{Ga}_{16 \mathrm{GUST} 44}$ [75]. Activation of this chimeric G-protein leads to a transient rise in calcium ion concentration within the cytoplasm. These calcium dynamics can be measured using ratiometric calcium sensors that are based on the FRET pair CFP and YFP connected by a calcium binding domain.

The principle of the sensor is depicted in Fig 1 . Since the array can contain hundreds of spots, it is possible to accommodate many different receptors and at the same time have a relatively large number of spots for each receptor type, leading to more precise estimates. For each spot on the array, fluorescence time series are measured at two different wavelengths which are, in a series of steps, transformed in response values for each spot upon exposure to a sample [100]. The goal of the statistical analysis, the main focus of this paper, is to be able to unambiguously and objectively discriminate between samples in terms of receptors affected.

\section{Material and Methods}

\section{Cell array experiments}

Reverse-transfected cell arrays were prepared and measured as previously described in [100]. The genes encoding bitter receptors were obtained from genomic DNA by PCR amplification and cloned into pcDNA3 containing the N-terminal sstr3 tag (gift from Dr. Wolfgang Meyerhof, German Institute of Human Nutrition Potsdam-Rehbrücke, Germany). Plasmid pcDNA3 Twitch2B (Addgene \#49531 [205]) was added to the print solution to enable ratiometric calcium detection. The arrays contained 24 individual bitter taste receptors, including SNP variants TAS2R4-SLN and -FVS, TAS2R38-PAV and -AVI, and TAS2R39-A and -T. For a complete overview, see - in the remainder of this paper, the TAS2 prefix will be deleted for clarity. These receptors were placed randomly on the array, to avoid location and neighbour effects. Each print mix contained $75 \mathrm{ng} / \mu \mathrm{l}$ receptor-coding plasmid DNA and $10 \mathrm{ng} / \mu \mathrm{l}$ calcium-sensor-coding plasmid DNA. The low level of sensor expression obtained this way prevented buffering of the calcium ions by the sensor itself. Two additional controls were printed; one control without receptor-coding DNA but an empty vector instead (Mock), and one control without receptor-coding DNA but with a modified calcium sensor protein that lacks the ability to bind calcium. This control, named YC-, has a YFP/CFP ratio independent of the intracellular calcium concentration. Any environmental influences on the spectral properties of the fluorescent calcium probe will be detected by this control, as will be fluorescence coming from the sample itself. 


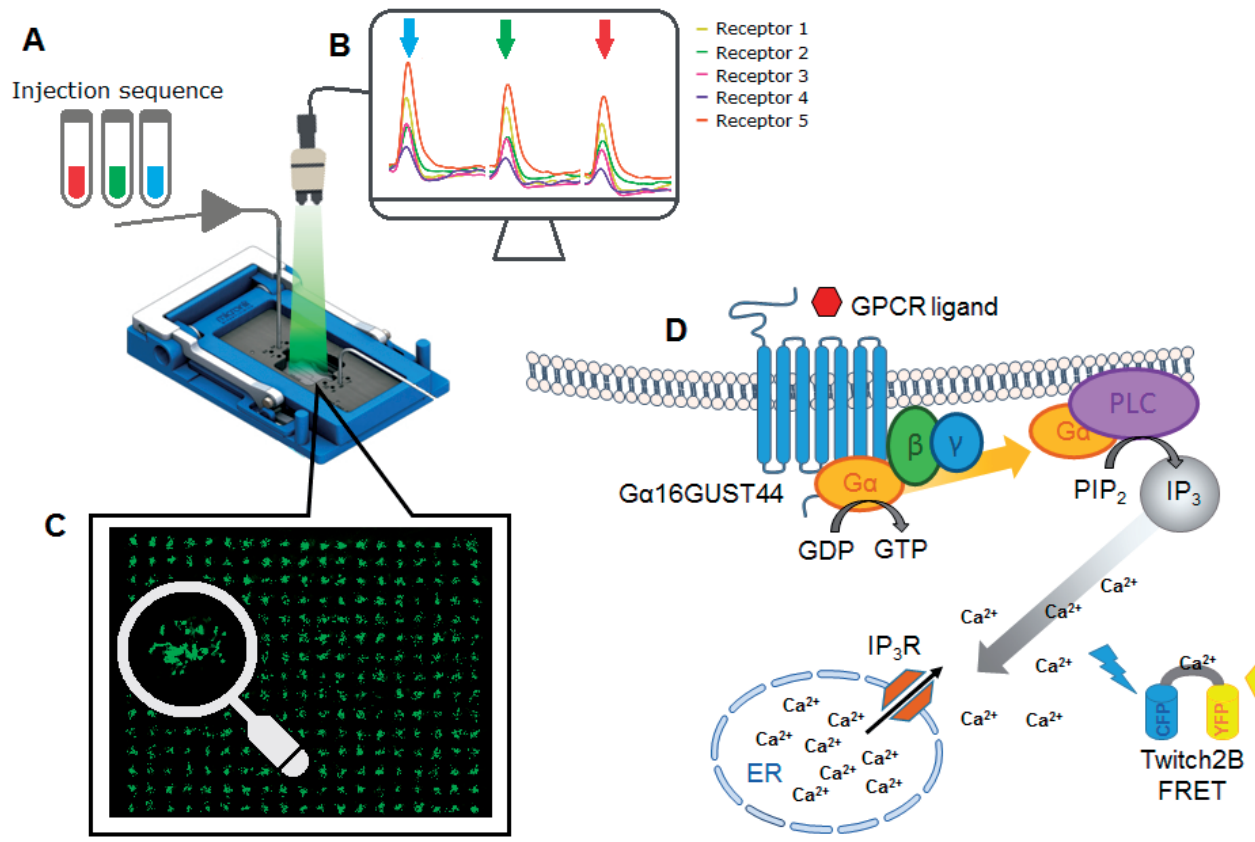

Figure 1, The receptomics principle used in this paper. The microfluidic system $A$ allows sequential injections of samples into the flowcell. The fluorescence microscope in B captures CFP- and YFPFRET images of the entire cell array that can be analysed to give average values for the YFP/CFP fluorescence ratio. $\mathbf{C}$ The spots of the array are composed of approximately 50 fluorescent cells, each transfected with a receptor-coding gene and a fluorescent calcium sensor coding gene. Both receptor and sensor are expressed in each fluorescent cell of an array spot $\mathbf{D}$. Each spot on the array expresses a different receptor type. When the receptor interacts with a ligand, the $\mathrm{G}_{16 \mathrm{GUSTA4}}$ signalling cascade is activated: the G $\alpha$ protein acts via Phospholipase $\mathrm{C}(\mathrm{PLC})$ to convert $\mathrm{PIP}_{2}$ into second Messenger $\mathbb{I P}_{3}$ which can interact with $\mathbb{P}_{3}$ receptors $\left(\mathrm{IP}_{3} \mathrm{R}\right)$ on the Endoplasmic Reticulum (ER). The $I P_{3} R$ are calcium ion channels which, when activated, transiently release calcium into the cytoplasm. The Twitch2B calcium sensor, expressed in the cytoplasm, is a fluorescent FRET probe which, upon binding of calcium ions, changes conformation and thereby increase the FRET efficiency between the CFP and YFP fluorescent proteins. These FRET changes are captured by the camera as a ratio change of CFP and YFP intensities.

Reverse-transfected cell arrays were prepared using HEK293 cells stably transfected with $\mathrm{Ga}_{16 \mathrm{GUST44}}$ (a gift from Dr. Takashi Ueda, Nagoya City University, Nagoya, Japan). At 48 hours after transfection, the cell arrays were removed from the incubator, washed and incubated in assay buffer for 1 hour prior to the measurements. All measurement series were performed using a $150 \mu \mathrm{l}$ flowcell and the flowcell holder (Micronit Microfluidics B.V., Fluidic Connect PRO Chip Holder.) The assay buffer ( $\mathrm{NaCl} 130 \mathrm{mM}, \mathrm{KCl} 5 \mathrm{mM}$, Glucose $10 \mathrm{mM}, \mathrm{CaCl}_{2} 2 \mathrm{mM}, \mathrm{HEPES} 10 \mathrm{mM}$ at $\mathrm{pH} 7.4$ ) was set to a continuous flow of $300 \mu \mathrm{l} / \mathrm{min}$ over the array. The injections were performed with a manual valve containing a $150 \mu$ l sample loop. Reagents used in the injections were Adenosine 5'-triphosphate (ATP, Sigma A6419), Chloramphenicol (Duchefa C0113.0100), Picrotoxinin (Sigma P8390), 6-propyl-2-thiouracil 
PROP (Sigma P3755) and D-Salicin (Wacko 199-00083). The time between injections was set to approximately 5 minutes, allowing the intracellular calcium levels in the solution to return to pre-injection values.

\section{Image analysis}

Upon stimulus by their respective ligands, receptors will induce a signal transduction leading to an increased calcium ion concentration in the cytosol. This can be monitored in real time by means of FRET (Förster Resonance Energy Transfer) imaging [100], here using a Leica fluorescent stereo microscope (Leica M205FA with DFC 345 FX camera). Two channels were monitored, CFP (ET CFP 10447409, excitation 436/20 and emission 480/40) and YFP (ET FRET 10450566, excitation 436/20 and emission 535/30), respectively. Note that CFP and YFP images are taken alternatingly and therefore have different time points. The CellProfiler software package [185] was used to separate signal pixels from the background to define a grid corresponding with the positions of the spots and to quantify the raw CFP and YFP signals.

\section{Pre-processing}

Processing the images leads to a data table containing, for each of the spots, the CFP and YFP signals for each measurement cycle. As a first pre-processing step, the data are corrected for fluctuations in the lamp output using a reference position outside the flowcell. Next, spots containing fewer than fifteen pixels are removed from the data. Receptor types that are represented by fewer than five spots are removed altogether. The CFP and YFP signals are then smoothed using cubic smoothing splines [232]. The signal of each spot, related to the calcium concentration in the cell, is now defined as the ratio of the interpolated CFP and YFP signals at specific time points. An example showing CFP and YFP data (after lamp correction) for one spot, as well as the derived spot signal, is shown in S1 Fig.

Next, this spot signal is used to calculate the response of a spot to a sample injection. This can be done in several different ways: since spot signals often show peak-like shapes, obvious candidates are peak height and peak area. Here, we focus on the the increase or decrease of a spot signal after the injection, given by the ratio of the extreme value of the signal (within a certain time window), and the average of the first three signal values directly after the injection. In this case, the time window is chosen to cover 30 cycles (approx. 1,5 minutes) directly after the sample introduction, corresponding to the time the cells are exposed to the sample. An example is provided by S2 Fig. In this way, a data matrix is obtained that describes the quantitative response of each spot to each sample injection, independent of the initial signal strength. 


\section{Statistical modelling}

Qualitative analysis - To obtain an easily interpretable overview of the differences between samples for which multivariate responses are available, Principal Component Analysis (PCA [233, 234]) is often used. In PCA, a high-dimensional data matrix is reduced to a much lower number of dimensions (for visualization purposes usually two) that contains the maximum amount of information. Each new dimension is a linear combination of the original variables. So-called score plots show the position of the samples in this reduced space; loading plots show the weights of the original variable on the new axes, i.e., which of the original variables are important in each new direction. Quantitative statements about significant differences between injections cannot be made with PCA. In a context where samples are compared to see whether they lead to different taste receptor responses PCA therefore is of limited value, and a statistical model is needed.

Mixed models - Here, we are interested in assessing which samples differ significantly from each other, and which receptor types are responsible for any observed differences. Our approach is based on linear mixed models [235]. In the simplest form, one fits a model describing the spot response $R$ with receptor and sample as (fixed) variables, also including the interaction between the two. Additional fixed variables could include the injection number, to account for any trends in sensitivity over time, and array number, when combining several replicated experiments in one analysis. To take account of the fact that every spot will have its own characteristics, the spots have to be part of the model, too. In order to avoid estimation of individual coefficients for all spots, which would consume valuable degrees of freedom, one can include spots as a so-called random variable. That is, one assumes that the spot effects follow a normal distribution around zero, and the only parameter that is estimated is the width of the distribution, implying that observations on the same spot are correlated rather than independent. This leads to the following model (in matrix notation):

$$
y=X \beta+Z u+\varepsilon
$$

where $y$ is the vector of spot responses to sample injections, $\beta$ contains the coefficients for all fixed variables, and $u$ contains the random effects associated with the individual spots. $X$ and $Z$ are design matrices relating the responses to the values of the independent variables: i.e. $X$ contains information about the sample type, receptor type and possibly injection number and array number, and $Z$ describes the receptor types present at all spots. Finally, $\varepsilon$ is the vector of residuals. The model is fit, as is common practice, through restricted maximum likelihood (REML) [235].

Contrasts - Once the model is obtained, it can be used to estimate the expected response of a particular type of receptor to a particular sample (so-called estimated marginal means). 
By focusing on differences of these estimates for individual spots, the differences between the spots are eliminated, removing a major source of irrelevant variation and leading to much narrower confidence intervals and increased statistical power. Such differences are called contrasts, and can be defined in a number of ways. In treatment-versus-control contrasts, for example, one of the injection types is used as a control, and the magnitude of the results of the other injection types (usually the study samples) is related to this. In this way one can quantitatively assess which receptors show different responses to the injection of different samples.

Scaling of the response variable - Although the mixed models can be fitted for the intensity ratio described above and shown in S2 Fig, it is more appropriate to use a logscaled intensity ratio as the response variable. Comparing samples then will (after backtransformation) lead to ratios of ratios: a value of 1 corresponds to no change in intensity ratio, values lower than 1 correspond to a decrease in intensity ratio, and values higher than 1 to an increase. In this way a treatment-versus-control contrast can be expressed in terms of simple ratios of the original CFP and YFP responses, which would not have been possible if we would have analysed the intensity ratio data without the log transformation. The variance-stabilizing effect of the log transform is less important here since all intensity ratios are relatively close to one; for other response variables with heteroscedastic residual variance, this could be an important reason to employ the log transform.

\section{Software}

All statistical analyses described in this paper have been performed using $\mathrm{R}$ [236], using packages $n / m e$ [237] for fitting the mixed models, emmeans [238] for obtaining estimated marginal means, contrast estimates and confidence intervals, and lattice [239] for generating plots. $\mathrm{R}$ scripts and data to reproduce the results in this paper are available in S1 Code, S1 Data, and S2 Data, respectively.

A stable version of the analysis scripts using defaults also used in this paper has been included in our "Receptomics" software, which provides an intuitive and powerful user interface allowing inspection of raw data, elimination of bad data points, choice of model ("raw" effect sizes or treatment-versus-control contrasts), and inspection of the outcome of the statistical modelling. For more information about this software, see http://www. receptomics.com.

\section{Data}

Three experiments, each executed three times, serve to illustrate our approach. In the first type of experiment (A), a quality control (QC) mixture of four compounds, chosen because they are known to hit specific bitter receptors, was injected at nine different dilutions (see Table 1). The sample with the lowest concentration was injected first; each subsequent 
Table 1, QC mixture and target receptors, in order of expected sensitivity [33]. Concentrations of compounds in the A-type experiments correspond to the most concentrated sample. The concentrations of the $\mathrm{QC}$ mixture in the B experiments correspond for all compounds but D-Salicin to $Q C 2$ in the $A$ experiments.

\begin{tabular}{llll}
\hline Spike & Exp A $(\mu \mathrm{M})$ & Exp B $(\mu \mathrm{M})$ & Affected receptors \\
\hline Chloramphenicol & 500 & 250 & R8 $>$ R46 > R1, R10, R43 \\
& & & $>$ R39 \\
Picrotoxinin & 500 & 250 & R14 > R46 > R1, R10 \\
D-Salicin & 10,000 & 1,000 & R16 \\
PROP & 20 & 10 & R38 PAV \\
\hline
\end{tabular}

Table 2, Injections for experiments A and B. The numbers after " $Q C$ " in Experiment $(A)$ indicate the dilution factors: samples have been injected in order of increasing concentration.

\begin{tabular}{lll}
\hline Injection & Experiment (A) & Experiment (B) \\
\hline 1 & Blank & Blank \\
2 & QC 256 & QC \\
3 & QC 128 & ATP 2uM \\
4 & QC 64 & ATP 2uM+QC \\
5 & QC 32 & ATP 2uM \\
6 & QC 16 & ATP 2uM+QC \\
7 & QC 8 & ATP 2uM \\
8 & QC 4 & ATP 2uM+QC \\
9 & QC 2 & ATP 2uM \\
10 & QC 1 & QC \\
11 & ATP 2uM & \\
\hline
\end{tabular}

injection had a double concentration of the QC mixture. In addition, a blank injection, and a $2 \mu \mathrm{M}$ ATP injection were performed. The ATP injection elicits a host-cell response, different for each receptor type. The second type of experiment (B) was set up to compare $2 \mu \mathrm{M}$ ATP injections with injections where the ATP sample was spiked with the same QC mixture as in (A). Also here a blank injection (containing only assay buffer) was included. This experiment is a simple example of a case where a specific response should be estimated in the presence of a constant background (the host-cell response). Table 2 gives an overview of the injections in both experiment types.

\section{Results}

\section{Experiment $A$}

Experiment type $A$ investigates spot responses to exposure to a dilution series, and was executed three times, using three identical arrays. The QC 8 injection in the first replication (experiment A1) did not succeed, so data for that particular dilution are missing. The PCA score plots for the three replicated experiments are shown in Fig 2. One can clearly see 
A1

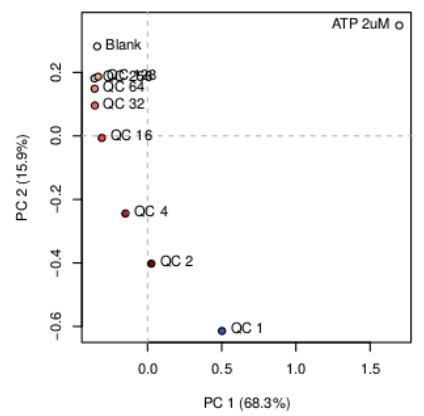

A2

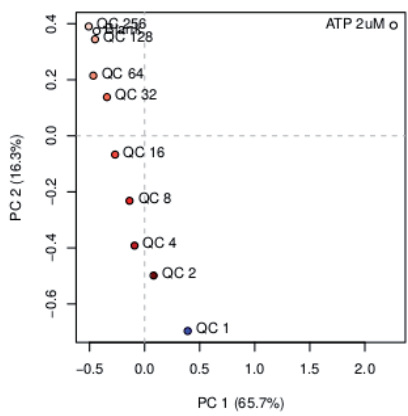

A3

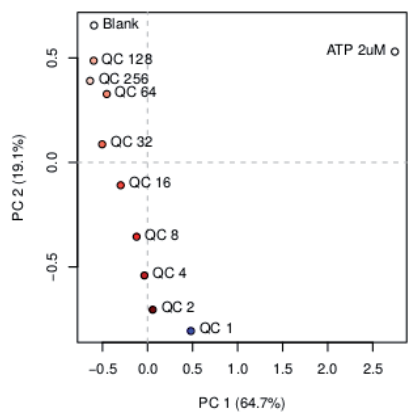

Figure 2, PCA score plots for the three replicated experiments, type A. Dots correspond to injections. In all three cases, the ATP injections lie in the top right corner, and the blank injections in the top left corner. Injections of the QC mixture move away from the blank injection with increasing concentrations.

the trend of more concentrated $\mathrm{QC}$ injections going away from the blank injection, while the ATP injection is in a completely different part of the PCA space. The corresponding loading plots, shown in S3 Fig, indicate which variables are involved in the first two PCA dimensions. Although some of the receptors, e.g., R8 and R14, seem to be important in PC2, the direction of increasing QC concentrations, the loading plots are hard to interpret because of the large number of variables and the fairly large variation, also within spots of the same receptor type.

More quantitative results are obtained with the mixed-models approach, presenting for each receptor type an estimate of the response to the different injections, including standard deviations and confidence intervals. By considering the treatment-versus-control contrasts, variability between spots is eliminated. These contrasts, estimated from the model combining the three arrays in one single analysis, are shown in Fig 3. The Blank injection is used as the control. In this figure, significant effects, not including the value of 1 in the confidence interval, are shown in red. In total, 42 significant effects are found. Going from top to bottom in each panel, concentrations of the QC mixture increase (i.e., dilution factors become lower) leading to larger responses for several of the receptors. Although the individual arrays show some small differences, the general patterns are very similar. Very clear responses are seen for receptors R8, R14, and R16, at somewhat higher concentrations also for R10, R38 PAV and R46L. All of these receptors are present in Table 1; receptors $\mathrm{R} 1$ and $\mathrm{R} 39$, also mentioned in the table, only show a significant response at the highest concentration. Note that some other receptors not present in Table 1 seem to respond, too - this may be a genuine response, since not for all receptors it is fully known what triggers them. The full results of the analysis of the individual arrays can be found in the supplementary material, S4 Fig - S6 Fig. 
Joint analysis for experiment $\mathrm{A}$

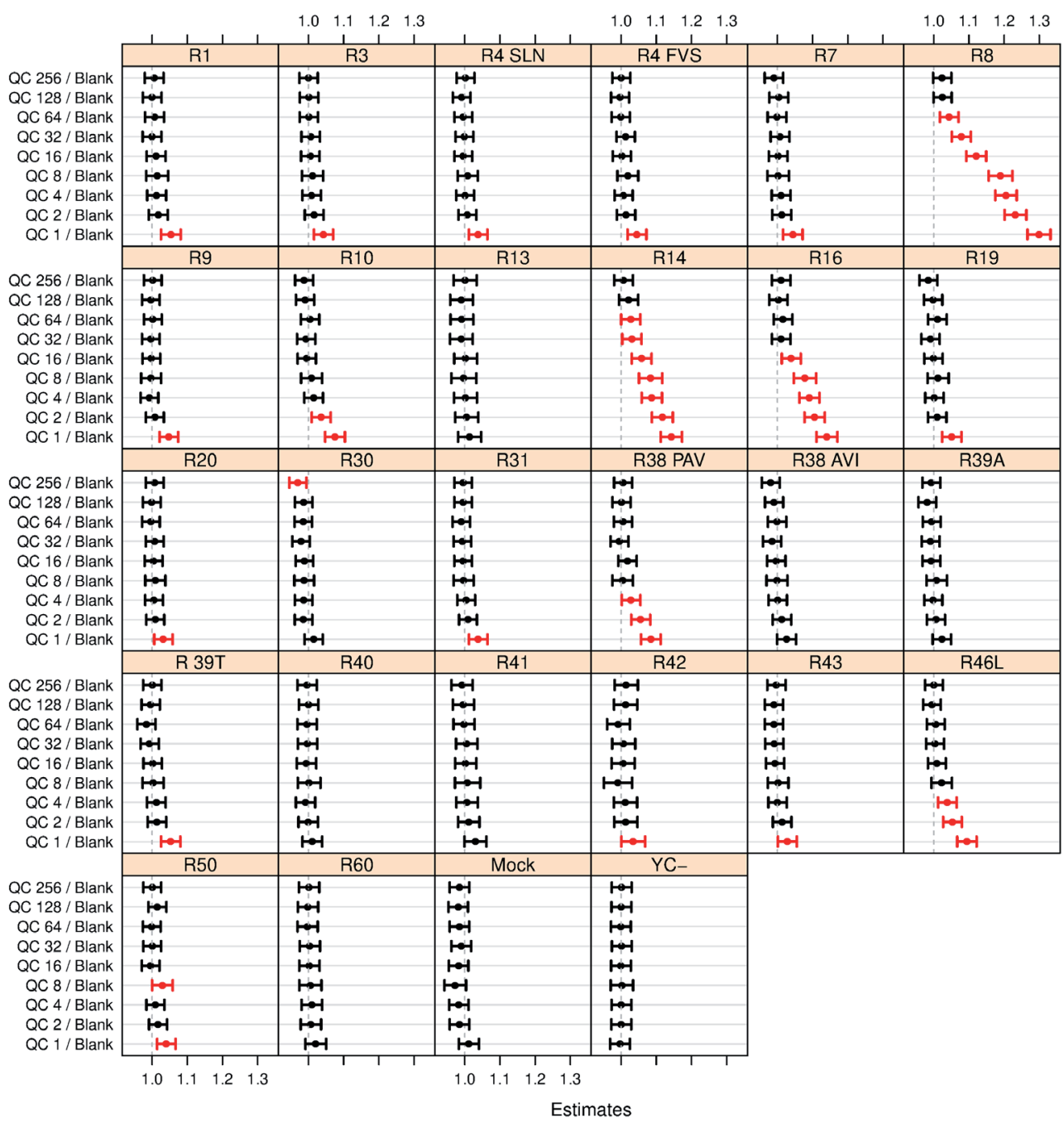

Figure 3, Control-versus-treatment contrasts from the joint analysis of the three type-A experiments. Each panel shows the estimated contrasts and associated $95 \%$ confidence intervals of a particular receptor. Contrasts are the ratio between the model response for a particular injection, and the reference injection, here the blank. Only the injections of the $Q C$ mixture are shown here to stress the pattern of increasing response with increasing concentration (from top to bottom, corresponding to decreasing dilutions indicated with the numbers on the y axis). Significant results, not including the baseline value of 1.0 in the confidence interval, are shown in red. For several receptors, e.g., R8 and R14, we see a clear relation between response and increasing concentration. 


\section{Experiment $B$}

In Experiment $B$ the goal is to distinguish between two types of injections, both containing a large host-cell response. This host cell response, independent of the transfected receptor, is set up by means of a sample containing ATP. One type of injection consists of the ATP sample; the other adds to this the QC mixture also employed in Experiment $A$, so that both a generic host-cell response and specific receptor-specific responses are elicited. The statistical model then should indicate which receptors show significant differences between the two types of injections.

The PCA score plots are shown in Fig 4. In all three replicated experiments, the four injection types (Blank, QC only, ATP only, and ATP plus QC) are located in four different quadrants. Clearly, it is possible to distinguish between the injection types: the first PC distinguishes on the basis of the presence or absence of ATP; the second PC does the same for the QC mixture. However, when looking at the loading plots (see S7 Fig) it is not at all clear which receptors are involved. The two most responsive receptors to the QC mixture, R8 and R14, are the ones with the biggest loadings on PC2, but we see appreciable differences, also within one type of receptor.

To be able to concentrate on the most relevant results only, the linear mixed models are constructed using only the ATP and ATP+QC injections, leaving out the Blank and QC injections. Peak number is included as a (numerical) variable, allowing a trend over time. This is necessary: in the score plots of Fig 4 the effect of the injection order is clearly visible. Later injections of the same type tend to have higher values on the PC2 axis.

The results for the first of the three experiments, B1, are shown in Fig 5. The two left panels show the estimated response values, basically averages of model predictions for the two different injection types, for all receptors. It is remarkable to see how the responses to the ATP injections vary widely between host cells carrying different bitter receptors. Perhaps even more surprising is the fact that the response of the Mock receptor to ATP is the strongest of all. The right panel in Fig 5 shows the treatment-versus-control contrast calculated by the mixed model, where the ATP $2 \mathrm{uM}$ injection is the reference. Since the comparisons are effectively made within each spot, the confidence intervals for the contrasts are much more narrow than the confidence intervals for the estimated responses, leading to increased statistical power. In total, 10 significant treatment-versus-control contrasts, not including the value of 1 in the confidence interval, are found here. Note that for both control receptors, Mock and YC-, the contrasts are not significant, indicating that the model has effectively eliminated the very large host-cell response component to ATP.

The estimated contrasts and confidence intervals for the three replicated $B$ experiments are shown in the top panels of Fig 6 . Even though the ATP leads to a large signal, varying 
B1

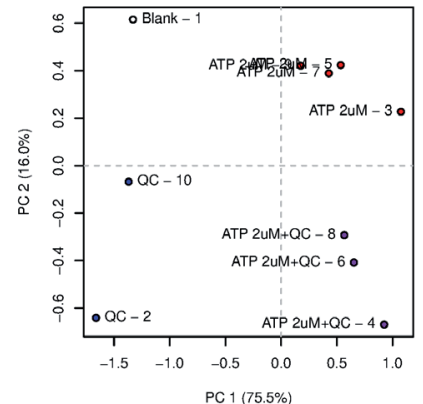

B2

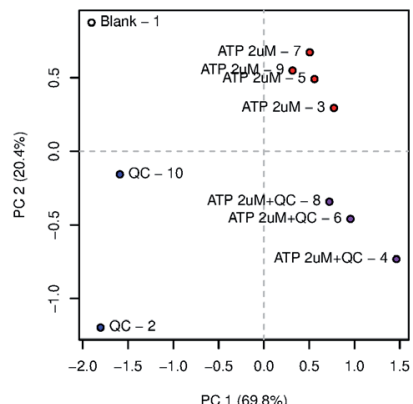

B3

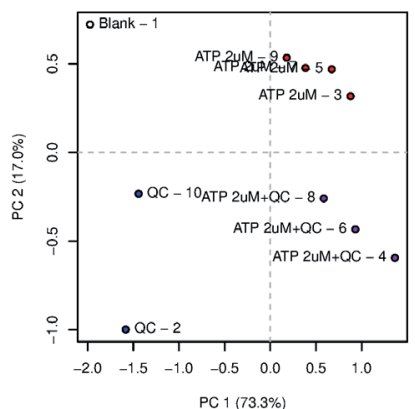

Figure 4, PCA score plots for the three replicated experiments, type B. Dots correspond to injections - the name of the injection type is followed by the injection number. In all three cases, injections without ATP are on the negative side on PC 1 ; injections without QCs are on the positive side on PC 2 . There is a clear trend where later injections are located more towards the top left corner of the plot.

B1
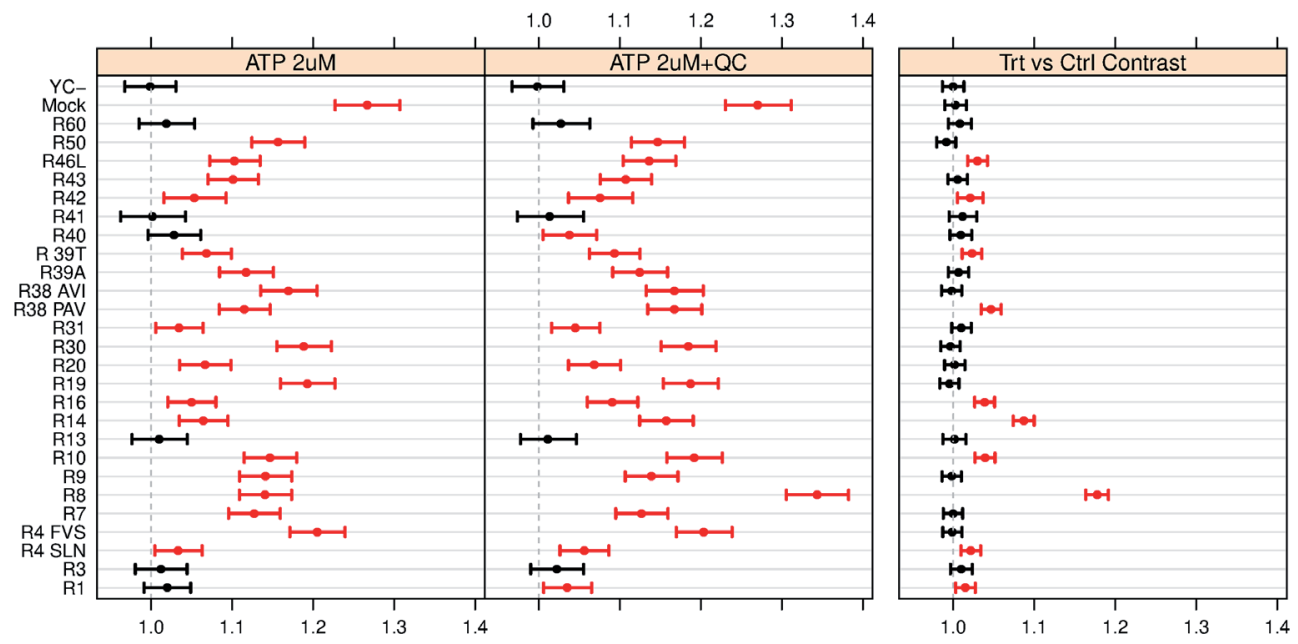

Figure 5, Estimates and 95\% confidence intervals for the first B-type experiment. The panels on the left show the estimated responses and associated confidence intervals of all receptors to injection types. The right panel shows the corresponding spot-wise ratios, the treatment-versus-control contrasts. Significant results, not including 1.0 in the confidence interval, are indicated in red. Note that the confidence intervals in the right panel are much more narrow than those in the left two panels since the between-spot variation is taken out of the equation.

in size depending on the type of the receptor, the analysis is able to identify the effects of the QC mixture on all receptors mentioned in Table 1 - six receptors, R8, R10, R14, R16, R38 PAV and R46L seem to stand out in particular. The joint analysis of the ATP-containing injections for the three experiments is shown in S8 Fig The two control receptors, Mock and YC-, overall have non-significant effects, as expected. 


\section{Experiment B - validation}

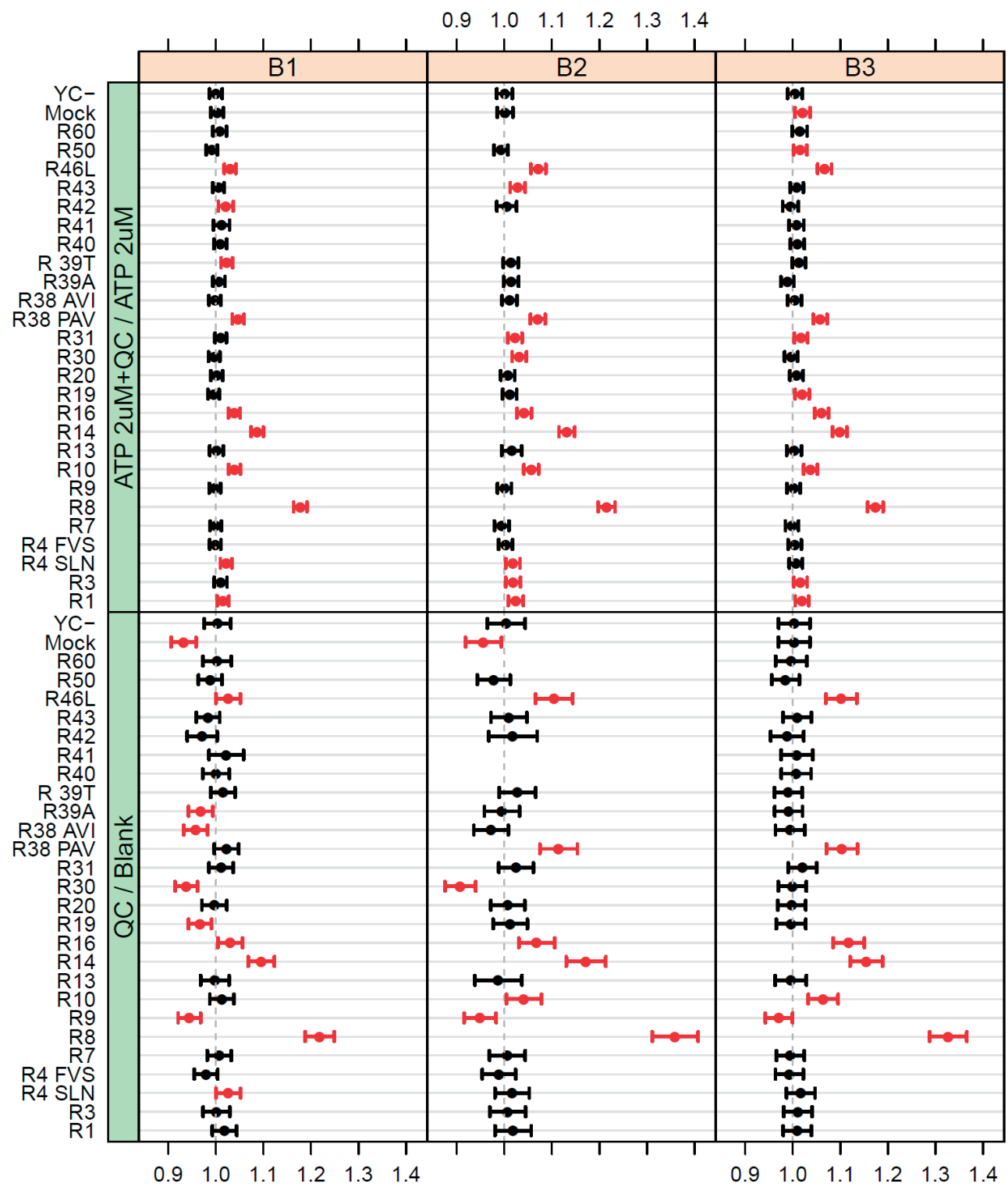

Estimates

Figure 6, Validation of mixed-model results in the presence of a strong background. The top panels show the estimated treatment-versus-control contrasts comparing spiked and non-spiked ATP injections. Even though the ATP will lead to a strong signal it is possible to pick up the receptors responding to the compounds in the QC mixture mentioned in Table 1. The bottom panels show the results of directly comparing the pure QC mixture injections with the blank, so without the ATP background signal. The top and bottom rows are generally in good agreement, even though the bottom panels show much more variation and wider confidence intervals due to the lower number of replicated injections. 
Corresponding results from the model fitted using only the Blank (the reference) and QC injections are shown in the bottom panels in Fig 6 . Even though this is supposedly a much easier task since the large ATP background is absent in this comparison, the results are much more variable: the contrast estimates themselves show large variation between repeated experiments, and the estimated confidence intervals are much wider. The main reason for this is that the replication level is lower: the blank was injected only once, and the QC mixture twice. In contrast, there are four non-spiked ATP and three spiked ATP injections. Nevertheless, this clearly shows that the mixed model is able to efficiently pick up signal also in the presence of a large background.

\section{Discussion}

Data from sensors based on live cells often lead to highly variable results. At the cell level, transfection efficiency and cell-cycle differences cause variation in protein expression, leading to variation at the spot level. This can be prominent if relatively few cells make up a signal on a receptor-cell microarray. In addition, in the analysis of complex samples both receptor-specific and generic host-cell responses are often encountered.

Here, these issues have been tackled in several ways. First of all, the design of the slide is important. The high replication of receptors of interest (typically, ten spots are printed for all receptor types) leads to more precise estimates. Perhaps even more importantly, a high replication provides a safeguard against "bad" spots - we are working with live cells so their behaviour is not always predictable. In the pre-processing phase a number of spots showing too little signal are typically eliminated. Since one of the main characteristics of the analysis pipeline proposed in this paper is to get rid of spot effects, we still should have enough spots for each receptor type to do this. For instance, for experiment B1, using only half the number of spots would on average lead to a $7 \%$ increase in the width of the confidence intervals and more variability in the contrast estimates. Note that the current array can easily accommodate all our bitter receptors and achieve high replication; it is also possible to simply print larger arrays containing more spots.

Secondly, quality control measures can be taken in the pre-processing, before the statistical analysis: removing spots represented by too few pixels in the raw images has already been mentioned. Sometimes air bubbles prevent spots from being covered by the fluid containing the sample - such spots would be flagged manually and removed from the analysis (this was not the case in the data presented in this paper). Additional quality control steps can easily be integrated if necessary. Thirdly, and the main topic of the current paper, by choosing an appropriate statistical model to describe the behaviour of the system very detailed and quantitative information can be obtained. The mixed models proposed here allow one to 
draw conclusions about whether or not two samples are different, and if so, what receptors are involved in sensing the differences between the samples. The results are given as tables including estimates and confidence intervals, and can be easily visualized. Of particular importance is the fact that the variation between spots of the same type, an important factor in the overall variability, can be separated from the relevant information by limiting the comparisons to be done within spots. In that sense, the mixed-model approach leads to more precise and more easily interpretable answers. This is true not only for the flow system described here but also in conventional methods using microtiter plates if repeated sample exposure could be achieved. Even the presence of a large generic host-cell response does not prevent the analysis from obtaining correct and precise results.

The current data-analysis set-up is very flexible and can be changed and adapted in several different ways. We can use other response variables, we can use more complicated models, or we can combine the results of the current models in a different way.

\section{Other response variables}

In this paper, the response of a spot is expressed as a ratio of the initial signal and the signal at the top of the peak. Obviously, other measures could have been chosen, such as the difference between the two signal values rather than the ratio. This would correspond to a simple measure of peak height. For the statistical analysis, however, this would lead to heteroscedastic data: high peaks would show much more variability than low peaks, necessitating more complex statistical models. A logarithmic transform of peak heights, which would at least partially alleviate the effects of such heteroscedasticity, is usually not possible since spot responses may be zero or even become lower after sample injection, leading to zero or negative peak heights. Therefore, our default is to use the log-scaled signal ratios described in this paper. Nevertheless, there may be cases where one is explicitly interested in fitting models for peak height. Also other response variables could be envisaged, such as the peak area, the degree of tailing of a peak, or the maximal steepness of the slope. Finally, in experiments where the timing of the response (early or late) is important one could, e.g., consider the time to reach the top of the peak, or to the start of the peak, as the response variable.

\section{More complicated statistical models}

The statistical model describing the behaviour of the system can be extended easily - this is one of the most attractive features of the current approach and a defining difference with the usual strategy of simply averaging spots of the same receptor type. One example of a simple extension is the linear term describing the decrease in response over a series of injections employed in the data from Experiment B. In this paper, we have used one slope to describe all spots, but one could actually fit receptor-dependent or even spotdependent slopes, or fit non-linear slopes such as an exponential decay. One could take 
into account interactions between subsequent injections - cells may respond differently to a stimulus depending on what happened in the near past. However, more complicated models obviously consume more degrees of freedom (and take more calculation time). The goal is to obtain an adequate description (using whatever definition appropriate) of the behaviour of the system with minimal resources. The set-up used in this paper takes a couple of seconds at most using simple every-day hardware, so is eminently usable in practice.

\section{More complicated comparisons}

We have shown that it is possible to obtain very good results in comparing spiked samples with non-spiked samples: the proposed methodology is able to eliminate the common background signal (triggered by ATP) from the receptor-specific signals, even though the background is the largest component by far. In more general experiments, where the differences between the injection types are more complicated than the simple addition of a spike mixture, it may not be so easy to remove host-cell responses: in pathological cases host-cell responses may completely cancel out receptor-specific responses. It is clear from the results in this paper that each receptor spot has a different host-cell response, and it is not correct to simply assume that the Mock spots provide a good estimate. We are currently exploring ways to disentangle the effects in a general way.

\section{Conclusion}

In the field of receptomics, microfluidic receptor-cell arrays are a valuable tool in investigating human responses to food and in trying to understand the relation between chemical composition and taste in food stuffs $[96,100]$. We have shown that careful experimental design, data processing and statistical analysis can lead to highly informative results, opening the way to many diverse applications. One particularly interesting possibility is to use these cell arrays as extensions of taste panels for pre-screening: in principle, many quantitative comparisons can be made in a rapid and cost-effective way. This receptomics tool can be further extended to other receptors of the GPCR gene family and ion channels, both of humans and other organisms.

Because of the complexity of the underlying biological processes, statistical analysis is non-trivial. In this paper, a consistent and robust strategy has been devised, validated and implemented in the form of R scripts to achieve maximum flexibility and transferability. On top of these scripts, a software package has been built, focusing on the models described in this paper, allowing also non-specialists to access the power of the mixed-model analyses, thus greatly reducing the time needed to analyse these complex data sets, and ensuring reproducibility of the results. 


\section{Author contributions}

Conceptualization: Ron Wehrens, Margriet Roelse, Maurice Henquet, Marco van Lenthe, Maarten A. Jongsma.

Data curation: Ron Wehrens, Margriet Roelse, Maurice Henquet.

Formal analysis: Ron Wehrens, Maarten A. Jongsma.

Funding acquisition: Maarten A. Jongsma.

Investigation: Ron Wehrens, Margriet Roelse, Maurice Henquet, Marco van Lenthe, Paul W. Goedhart, Maarten A. Jongsma.

Methodology: Ron Wehrens, Paul W. Goedhart, Maarten A. Jongsma.

Software: Ron Wehrens, Paul W. Goedhart.

Supervision: Ron Wehrens.

Validation: Ron Wehrens.

Visualization: Ron Wehrens, Marco van Lenthe.

Writing - original draft: Ron Wehrens, Margriet Roelse, Maarten A. Jongsma.

Writing - review \& editing: Ron Wehrens, Margriet Roelse, Maurice Henquet, Marco van Lenthe, Paul W. Goedhart, Maarten A. Jongsma.

\section{Data Availability Statement}

All data discussed in the paper are provided as supplementary material.

\section{Funding}

This work was supported by grant numbers Topsector Horticulture \& Propagation materials KV 1409-025 (received by MAJ). The URL to sponsors' website is https://topsectortu.nl/ $\mathrm{nl} /$ high-throughput-phenotyping-tomato-flavour. The funders had no role in study design, data collection and analysis, decision to publish, or preparation of the manuscript.

\section{Online data}

\section{S1 Code.}

scripts.R: file containing $\mathrm{R}$ scripts for reproducing the results (including figures) in this paper. https://doi.org/10.1371/journal.pone.0214878.s002

\section{S1 Data.}

ExperimentA.csv: file containing data for the three experiments of type A. https://doi. org/10.1371/journal.pone.0214878.s003

\section{S2 Data.}

ExperimentB.csv: file containing data for the three experiments of type B. https://doi. org/10.1371/journal.pone.0214878.s004 


\section{Supplemental data}

Table S1, Overview of bitter raste receptor genes used in the bitter-receptor array. Polymorphism variants are indicated with the aminoacid number of the receptor protein and the variable aminoacid letter code. In brackets the printed polymorphism is shown. The rightmost column contains the number of replications on the array.

\begin{tabular}{llll}
\hline Receptor number & Name & Polymorphisms & $\#$ \\
\hline 1 & TAS2R1 & & 10 \\
2 & TAS2R3 & F7S, V96L, S171N (FVS) & 10 \\
3 & TAS2R4 & F7S, V96L, S171N (SLN) & 10 \\
4 & TAS2R4 & & 10 \\
5 & TAS2R5 & & 10 \\
6 & TAS2R7 & 10 \\
7 & TAS2R8 & & 10 \\
8 & TAS2R9 & & 10 \\
9 & TAS2R10 & 9 \\
10 & TAS2R13 & 10 \\
11 & TAS2R14 & & 10 \\
12 & TAS2R16 & 10 \\
13 & TAS2R19 & 10 \\
14 & TAS2R20 & & 10 \\
15 & TAS2R30 & 10 \\
16 & TAS2R31 & & 10 \\
17 & TAS2R38 & & 10 \\
18 & TAS2R38 & 10 \\
19 & TAS2R39 & A49P, V262A, I296V (PAV) & 10 \\
20 & TAS2R39 & A301T (A) & 10 \\
21 & TAS2R40 & A301T (T) & 10 \\
22 & TAS2R41 & & 10 \\
23 & TAS2R42 & & 10 \\
24 & TAS2R43 & & 10 \\
25 & TAS2R46 & & 10 \\
26 & TAS2R50 & & 10 \\
27 & TAS2R60 & & 10 \\
29 & Mock & & 10 \\
\hline & YC- & & 10 \\
\hline
\end{tabular}



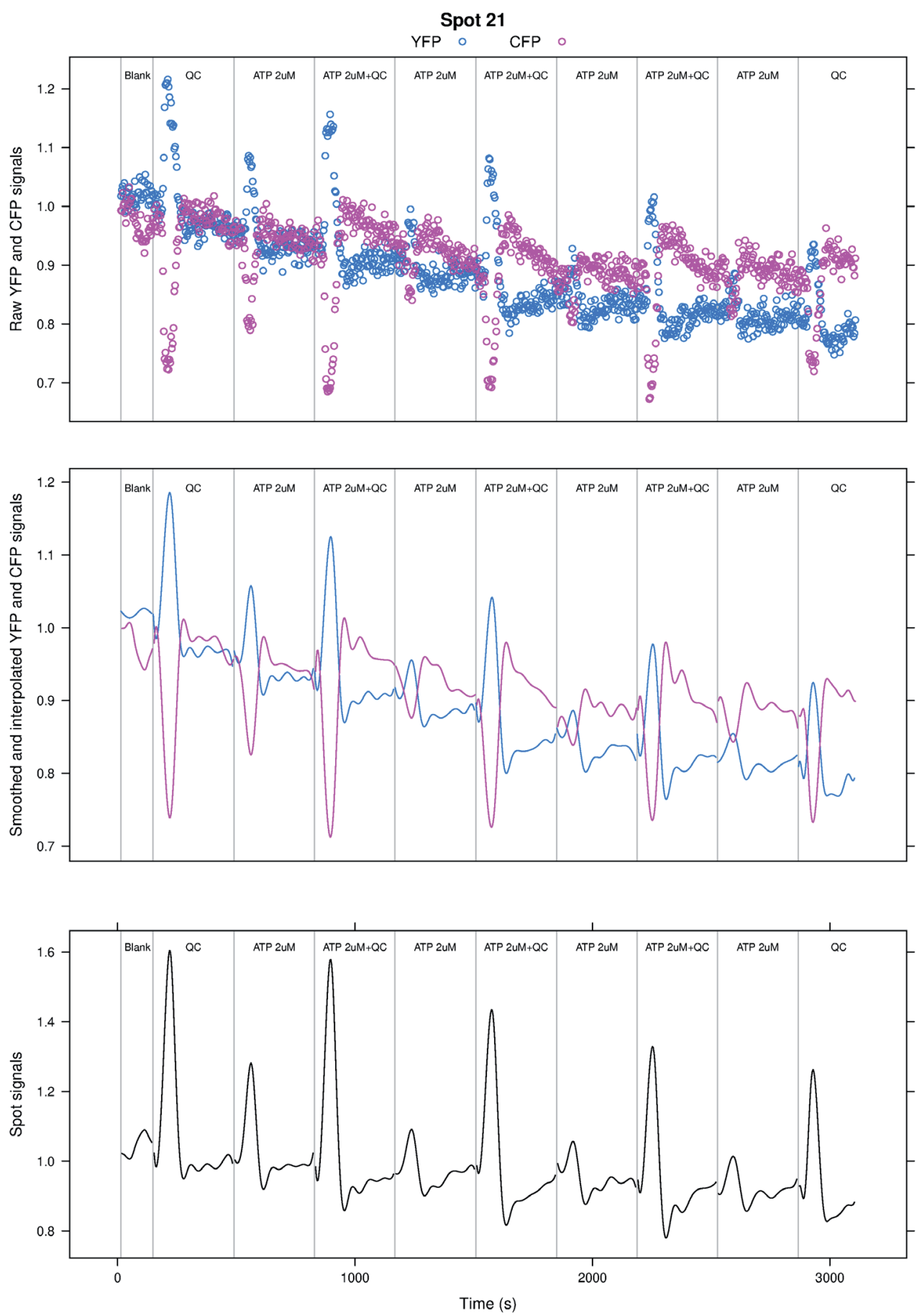

Figure S1, Data processing: from CFP and YFP signals to spot signals. The top panel shows the raw CFP and YFP signals for spot 21 from the first type-B experiment (a spot of receptor type R8). These raw signals are smoothed and interpolated to obtain values for exactly the same time points (shown in the middle panel). The final spot signal is calculated as YFP/CFP, and is shown in the bottom panel. 


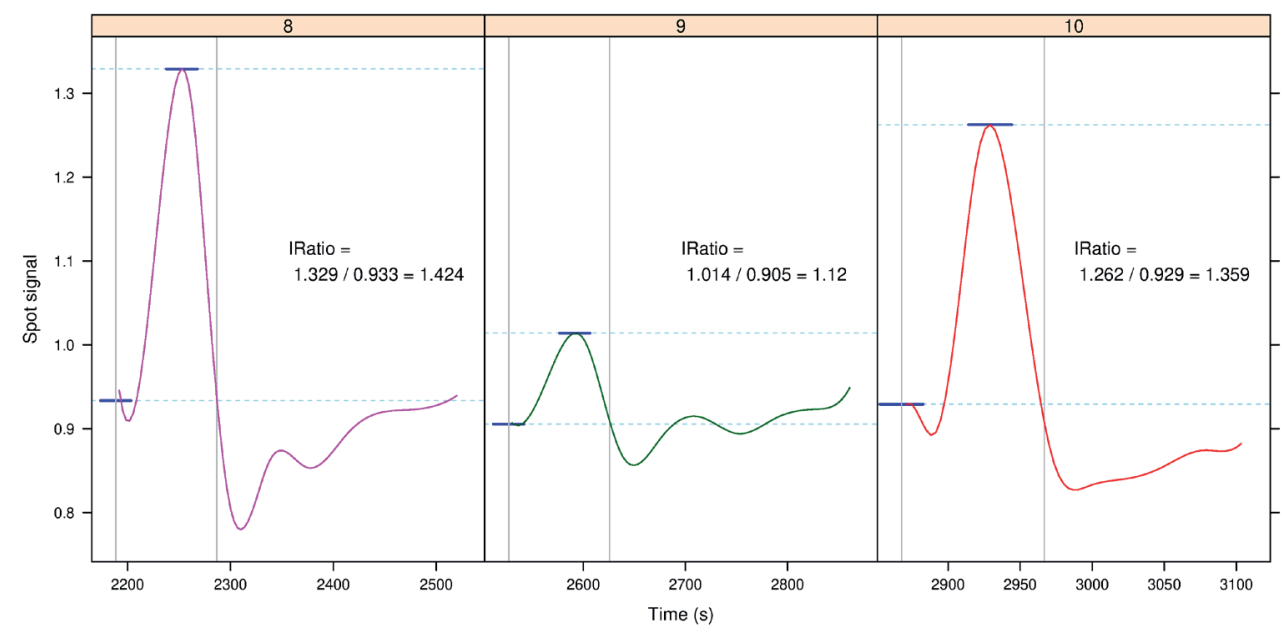

Figure S2, Definition of the magnitude of the spot response to an injection. The plots depict the last three injections of the data also shown in S1 Fig. The magnitude of the spot response is the ratio of the extreme point within a time window (here 30 cycles, indicated by the gray vertical lines), and the starting value, the average of the first three points.

A1

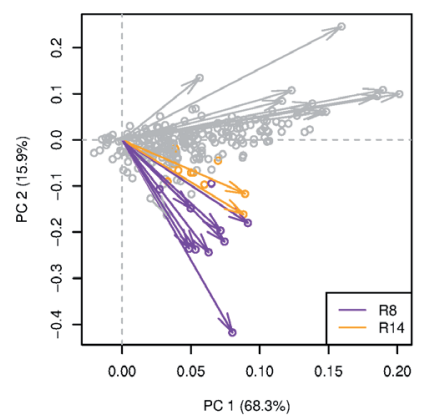

A2

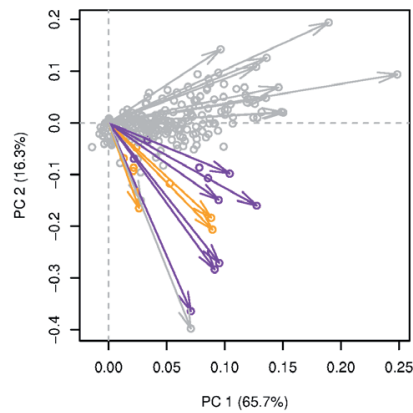

A3

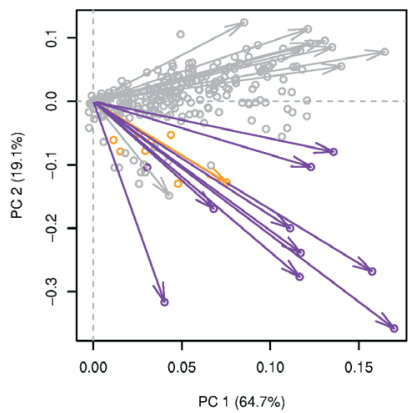

Figure S3, PCA loading plots for the three replicated experiments, type A. Spots of types R8 and R14 are highlighted to show the variability between spots of the same receptor type. For clarity, the largest loadings are shown with arrows, smaller ones are shown with dots only. 
A1

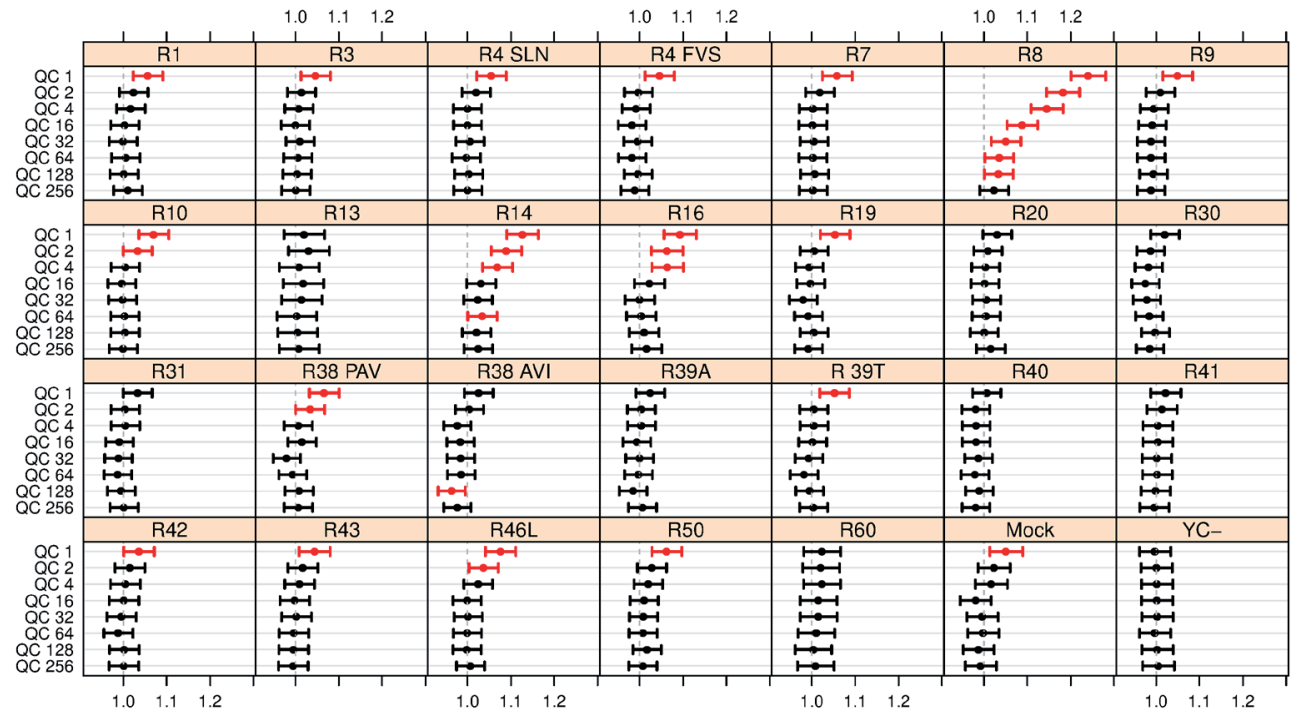

Figure S4, Estimated treatment-versus-control contrasts for individual spotmixes in Experiment A1. Each panel contains the result of one receptor type; dilutions are given at the $y$ axis, with stronger dilutions towards the bottom. Significant contrasts, not containing the value of one in the confidence interval, are indicated in red. The blank injection serves as the reference.

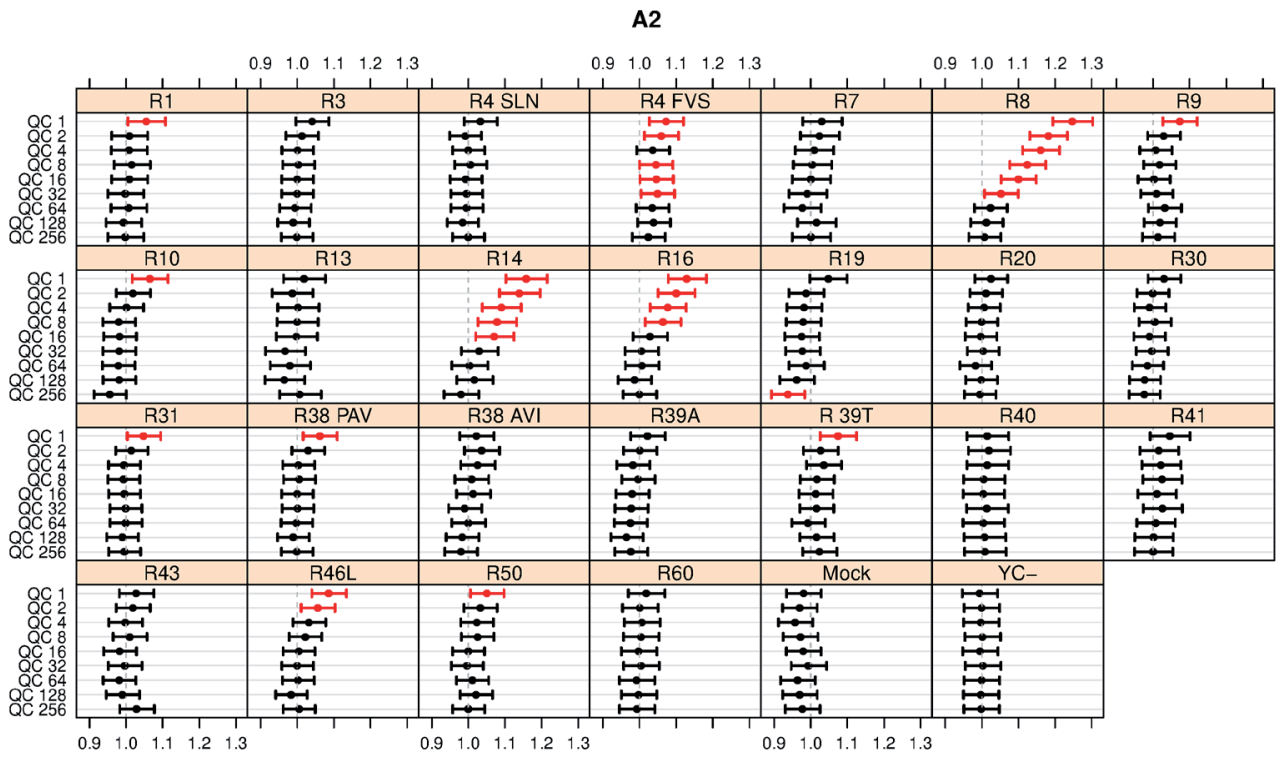

Figure S5, Estimated treatment-versus-control contrasts for individual spotmixes in Experiment A2. For explanation, see legend of S4 Fig. 


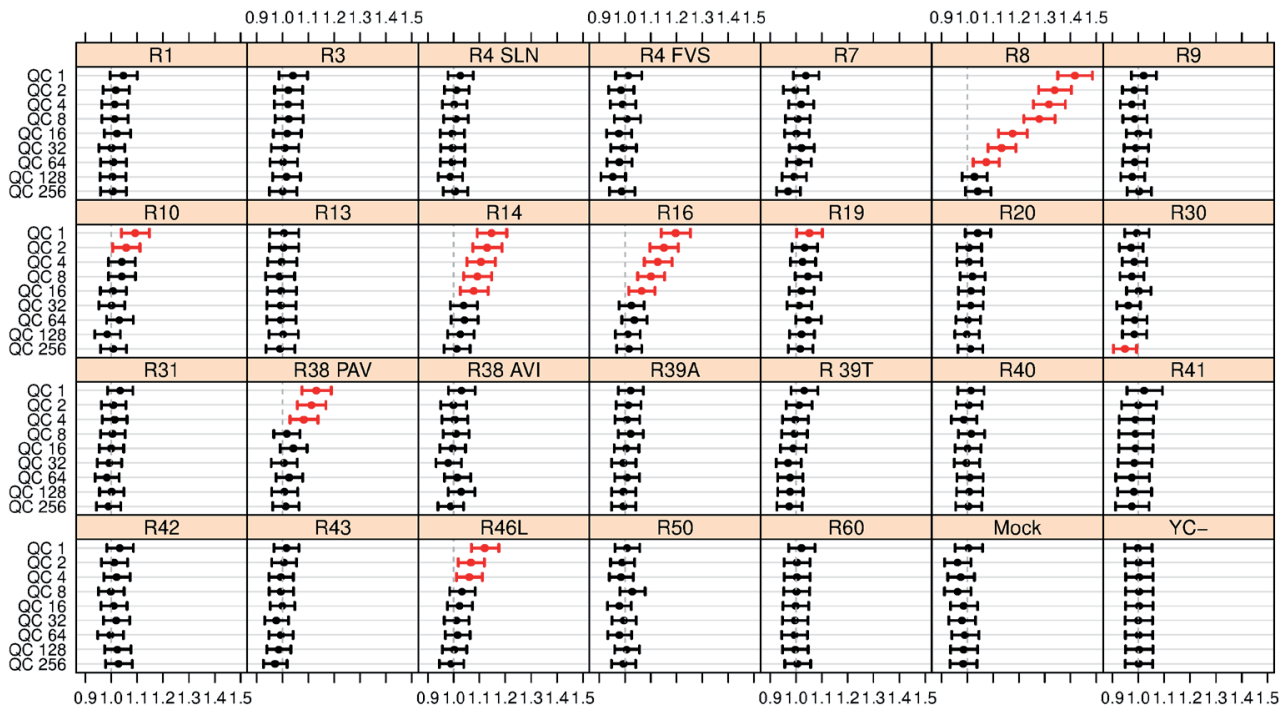

Figure S6, Estimated treatment-versus-control contrasts for individual spotmixes in Experiment A3. For explanation, see legend of S4 Fig.

B1

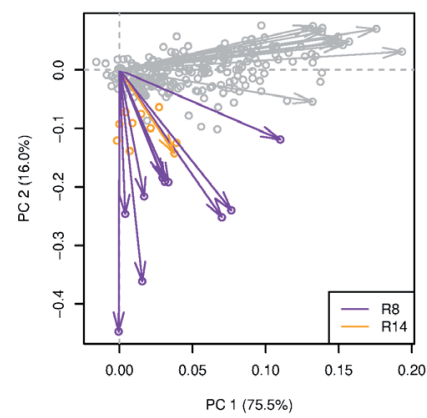

B2

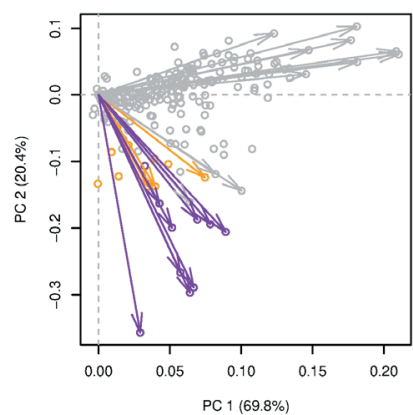

B3

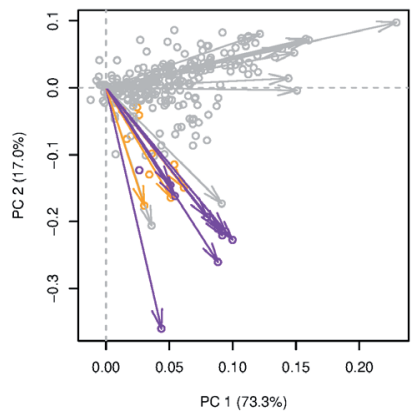

Figure S7, PCA loading plots for the three replicated experiments, type B. Spots of types R8 and R14 are highlighted to show the variability between spots of the same receptor type. For clarity, the largest loadings are shown with arrows, smaller ones are shown with dots only. 


\section{Joint analysis for experiment B}

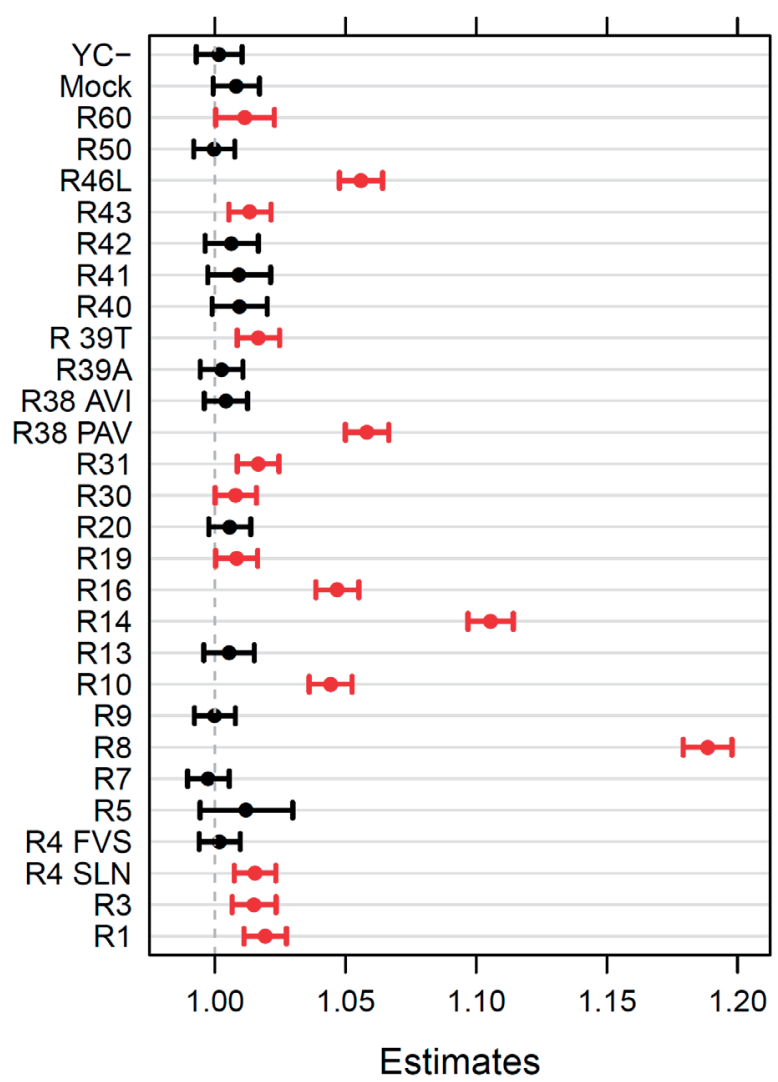

Figure S8, Joint analysis of type-B experiments. For explanation, see the caption of Figure 3 . The reference level is given by the injection type ATP $2 \mathrm{uM}$. 

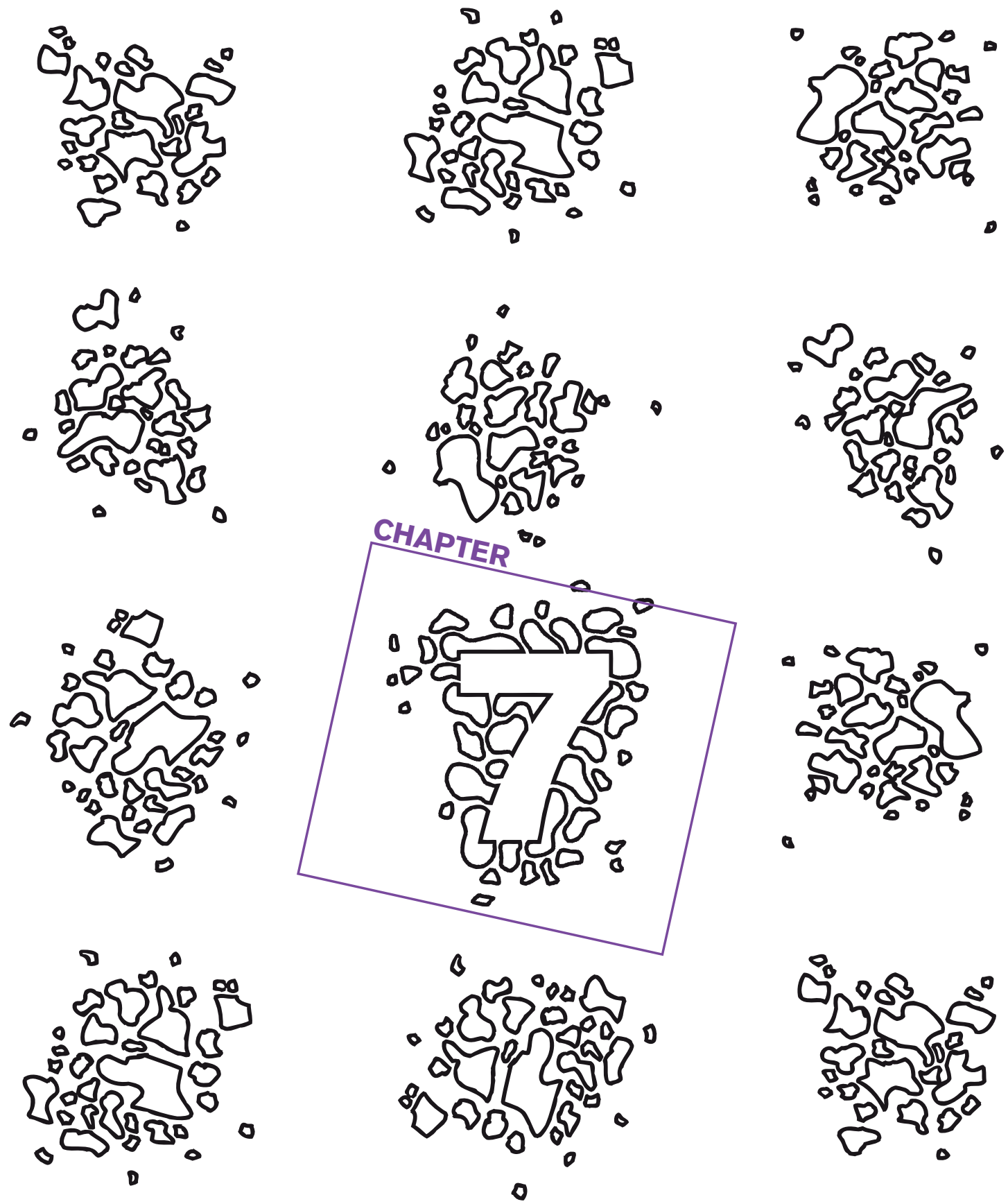

0
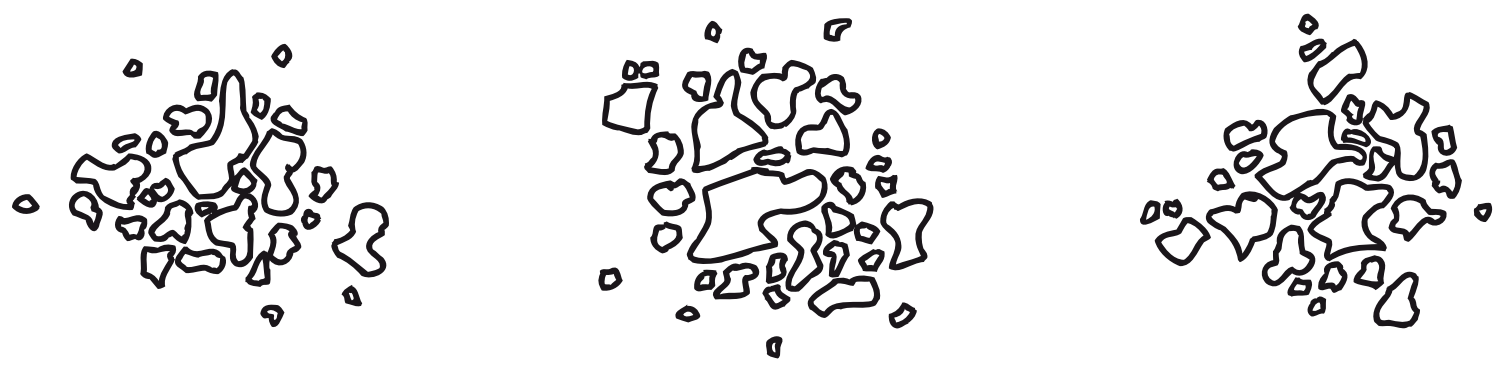
General discussion 
The receptomics concept as presented in this thesis involves a microfluidic screening platform that captures the response of a receptor cell array during sequential exposure of samples. This receptor cell array can potentially study the many different membrane receptors of an organism, hence the true -omics nature of the technology. In this last Chapter, I will elaborate on the current state and potential future development of this technology. Furthermore, I will summarize the challenges and the potential solutions to overcome these.

\section{The potential impact of receptomics}

As shown in the previous chapters, receptomics holds promise as a high-content receptor screening technology with specific advantages compared to currently used screening strategies.

Conventional receptor screening technologies generally involve end-point assays. These assay types are currently the gold standard for industrial and pharmaceutical high throughput functional receptor screening. An end-point assay can be compared to a single test-tube reaction. All ingredients are mixed and the resulting outcome is measured as a "total well" response. A typical end-point receptor assay is based on a multi-well plate containing cells, transiently transfected with a DNA construct containing a receptor-coding gene and a reporter-based read-out. After adding test samples to the wells, the wells are ideally measured in parallel for a response [90]. Such screening assays can be performed relatively quickly and are easily automated (e.g. FLIPR assays). Compound libraries are routinely screened against a limited number of molecular targets, often receptors or enzymes. Methods are well established, technically robust and often utilized by large companies in the pharma and flavour sectors [240]. Notwithstanding their obvious advantages, these conventional screening platforms carry a number of limitations. The technology described in this thesis combines a receptor cell array with a microfluidic set-up, thus enabling a dynamic assay rather than a static or end-point assay. It allows the sequential analysis of samples while studying interactions with multiple receptors in parallel. This comes along with additional benefits in terms of cost savings by reducing sample volumes and flexibility towards sample exposure and testing order.

\section{Receptomics, a microfluidic assay}

The most fundamental difference between receptomics assays and end-point assays is the implementation of a microfluidic system and sequential exposures of the receptor cell array. While in an end-point assay the reagent and test mixture remain in the assay well, the receptomics cell array, as developed in this thesis, is exposed to the samples in a controlled way. The microfluidic system which continuously washes assay buffer over the 
cell array is combined with an injection system to inject pre-determined volumes of sample into the flow thereby controlling the duration of sample exposure. Microarray technology and microfluidics are generally acknowledged as a promising combination as for example outlined by Sofia Martins in her review on GPCR-based live-cell screening assays [94]. Compared to a static, end-point assay, a receptomics assay carries, both advantages and disadvantages as outlined below.

\section{Advantages:}

1. Reduction of production and running costs. Preparation of reverse transfected cell arrays for a receptomics analysis requires 100x less DNA and transfection reagent while no fluorophore dyes are used. The slides and flowcells are reusable omitting the use of disposable plastics, while the currently-used imaging setup is more economical in terms of acquisition costs compared to that with fully automated plate readers.

2. Sample quantity needed to screen an entire receptor library can be reduced ( 10 100x) using microfluidics compared to an end-point assay. This is a significant advantage when the sample volume/quantity is limited (for example when using LCMS fractions) or costly.

3. In an end-point assay the sample remains available to the receptors after it is added to the well, and one basically studies continuous exposure of a single application. However, continuous exposure may in itself obstruct the observation of relevant aspects of the responses of some receptors, including signal lingering, baseline increase or decrease, inactivation of both the receptor and $\mathrm{G}$ protein by kinases and arrestins, secondary responses etc. By contrast, controlled and repeated exposure in a microfluidic system enables the study of such secondary effects.

4. When cytotoxicity is an issue, lowering the exposure time will cause less cell stress and still allow screening for receptor specific responses. In a multi-well assay the sample concentration can be controlled, but the exposure duration is continuous. In a microfluidics system, the exposure duration can be tightly controlled and even reduced to pulses of just a few seconds [95].

5. Microfluidics allows for sequential dosing experiments. A sequential dosing with controlled concentration, duration and frequency is better able to mimic physiological conditions at the cellular level and thus provides a more realistic setting for, for example, organ-on-a-chip (nose, tongue, gut) type platforms. For instance, the rise and fall of glucose levels, hormone secretions and immune responses all provide sensing cells with a rhythm of activations [241]. The frequency and amplitude of these activations determine the extent and mode of cellular responses which may vary from transcription regulation, hormone secretion, depolarization etc. Studies on the biochemical circuit architecture of receptors in cell lines have profited from the development of microfluidic systems that allow repeated temporal exposures [242]. 
6. Last but not least, the implementation of microfluidics allows the coupling of different compartments or modules. An example of this could involve a lab-on-a-chip platform where sample handling like mixing or gradients of samples are easily programmed. An organ-on-a-chip platform could be designed by connecting organ modules and cell array modules to sense, for example, the excretion of endogenous ligands such as hormones and cytokines. The coupling of modules could also be extended to combine different analytical platforms with the receptomics array. In Chapter 3 we propose the direct coupling of an LC-MS system with receptomics to create on online bioactivity screen. In one chromatography run, a complex extract could be separated into its components which are subsequently detected and identified by mass spectroscopy upon elution from the column, and, in parallel, continuously screened for receptor-ligand binding activity. Figure 1 gives an overview of some different microfluidic assemblies that can be envisioned.

\section{Disadvantages:}

1. Memory effects of previous exposures. Cultured cells are highly complex and continuously process many factors based on their physiological state. Exposing cells to both pure and complex matrices will therefore change conditions beyond experimental control. Confirmation from both positive and negative controls is crucial to provide context to the experiments.

2. Exhaustion of the receptor response. Exposure to saturating levels of ligands causes phosphorylation by kinases of both the receptors and the $\mathrm{G}$ proteins. As a result, the receptors are for example targeted by the beta arrestin pathway and then internalized. This leads to a loss of response signal in subsequent exposures $[42,103]$. Furthermore, different receptors differ strongly in this respect. These desensitizing effects need to be taken into account and require control injections of known agonists to monitor the receptor exhaustion dynamics $[243,244]$.

\section{Receptomics as a high content screening platform}

As mentioned above, the traditional end-point assays have evolved into highly efficient compound screening assays. Their high-throughput nature - due to advances in automation in this field - makes them very efficient for screening large libraries of compounds against a few target receptors. But also, vice versa, compounds may be screened against receptor libraries in a multi-well plate format. This type of receptor library screening is offered commercially making a receptor-omics type screening feasible also in the conventional system.

The end-point assay is also very efficient in screening for/discovery of response modulators like blockers and enhancers for target receptor-agonist combinations. In this case, a well is pre-incubated with a modulator and the agonist is then added. This method is called a 2-addition assay and may be used to study agonist, antagonist or partial agonist effects [91]. 
A

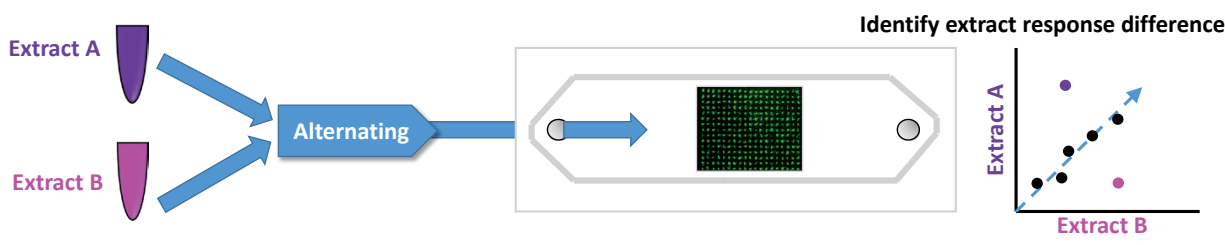

B

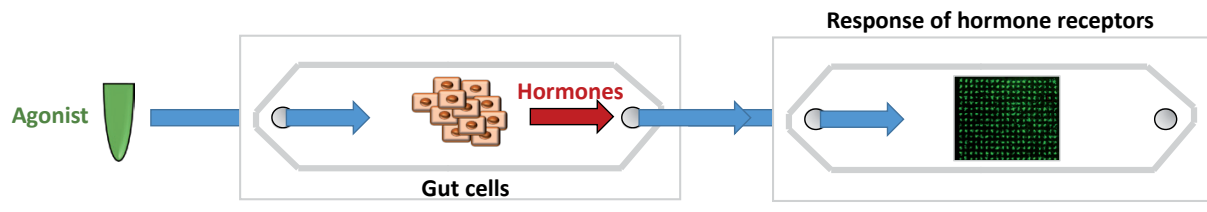

C

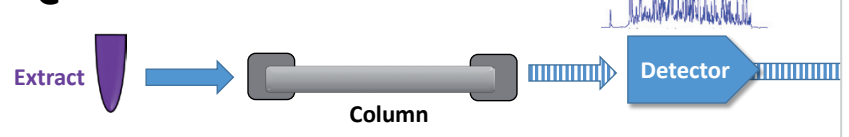

Detection of bioactivity

Figure 1, possibilities for microfluidics-based research in combination with a receptor cell array. A, By alternating injections of two unknown samples or extracts, the receptors that are responding differentially to one extract can be analyzed in a direct comparison or contrast between the extract responses. B, Coupling two flowcells in series. The first flowcell can be used to culture a specialized organ tissue and the second flowcell can containing a receptor array to measure real-time cellular responses from e.g. secreted hormones. C, Coupling of an LC-MS system to a receptor cell array and detect bioactivity in peaks as they elute from the column (see next paragraph for a more detailed perspective)

Our microfluidic assay, in combination with a cell array, offers a different perspective on the screening of response modulators. It may best be used to study the effect of known response modulators to several receptors simultaneously. For example, bitter taste modulators may have different modulating effects on different bitter receptors. Some compounds have antagonistic effects on one bitter receptor but have agonistic effects on another bitter receptor. Sequentially exposing a full array of bitter taste receptors to alternating samples of agonists and agonists combined with known modulators would yield extensive information on the modulating effects on all bitter receptors. Obtaining such insights would be much more laborious when using multi-well plate platforms.

The receptomics platform cannot compete with the existing high throughput receptor screening technology in terms of rapid screening of sometimes several thousands of potential ligands from a compound library. Instead, it offers a new paradigm; the microfluidic assay advantages as mentioned above can be employed to facilitate new directions of research.

Figure $1 \mathrm{~A}$ shows how the sequential injection nature of the receptomics assay allows the study of inter-sample differences. With several alternating and repeated injections, a spot- 
based response trend can be discovered showing these inter-sample differences based on a linear mixed model. This spot-based data analysis (see Chapter 6) considers each spot as a separate measurement. And because each spot is serially exposed, this allows us to draw conclusions about differences between samples and disregard the variability between spots. In this way we can separate the biological variability between spots of the same type from the relevant information, that is, the variation in responses between two samples. The two samples may for example be extracts of two plant varieties, a reference and a test product or an agonist in the presence of absence of a response modulator (antagonist, allosteric modulator, inverse agonist etc.). This strategy allows the receptomics system to discover sample differences even in samples that carry factors that induce a host cell response (ECRO 2018 conference poster [245]).

In Figure $1 \mathrm{~B}$ and $\mathrm{C}$ the receptomics platform is coupled to another type of platform, for example, an organ on a chip (B) or an LC-MS (C) system. The microfluidic nature of the platform allows the coupling with other microfluidic platforms, thereby potentially gaining advantages in terms of assay speed, costs and the sample quantity needed for the assay.

\section{Perspectives on the coupling of LC-MS to receptomics}

There is an increasing interest in natural extracts for use in food and medicine. Such extracts have complex compositions and the bioactive components are often unknown. The characterization of the bioactive compounds in an extract is costly and time consuming. Often, crude extracts from plants with strong bioactivity can be significantly diluted without loss of relevant receptor responses.

The receptomics technology is efficient for screening receptor libraries with small amounts of sample as in the case of LC-MS fractions (see Chapter 3), and the flow cell feature of the system creates the unique opportunity for direct coupling to other microfluidic platforms like liquid chromatography MS. Such fluidic coupling would enable simultaneous identification of the molecular mass and any specific bioactivity of compounds eluted from the column. This one step process, as shown in Figure 2, could greatly accelerate bioactive compound discovery.

To answer the question whether LC-MS-receptomics is feasible by coupling the eluent (waste) of an LC-MS directly to the receptomics platform as suggested in Chapter 3, a pilot study was performed that aimed at monitoring the cell responses under physical and chemical conditions that may be expected in such a setup. In this study we chose to focus on compatibility with acetonitrile (ACN) and formic acid (FA) in the running buffer since ACN and FA are commonly used organic solvents to elute LC columns. ACN concentrations may go as high as $60 \%$, therefore, to decrease cytotoxicity, we propose at least 100 fold dilution of the eluent. In addition, we looked at the effects of increasing the flow rate to a speed 


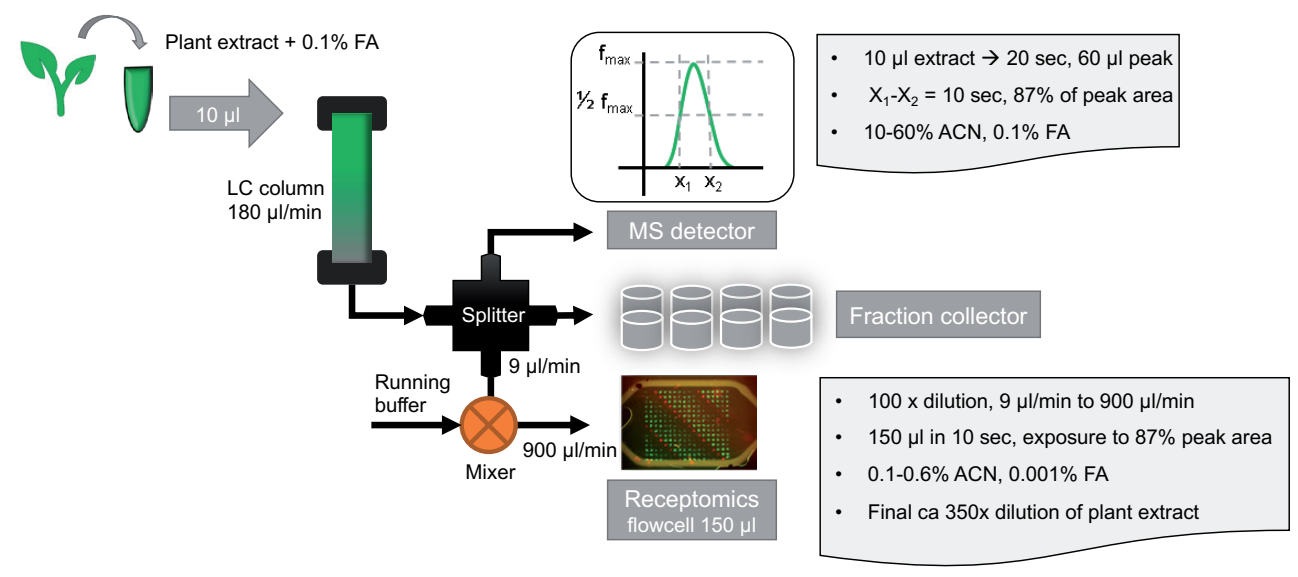

Figure 2, Process diagram of the coupling of a receptor cell array to the eluent of an LC-MS. A regular LC-MS set up with a standard column can be injected with a maximum of $10 \mu \mathrm{l}$ sample. At a standard flow rate of $180 \mu \mathrm{l} / \mathrm{min}$, this will broaden from a peak width of 3.3 seconds $(10 \mu \mathrm{l})$ to a peak width of 20 seconds and a corresponding volume of $60 \mu$ (Ric de Vos, Wageningen UR, personal communication). The most relevant high concentration part ( $87 \%$ of the peak area) is contained in a window of 10 seconds and $30 \mu \mathrm{l}$. The peak is part of a continuous flow from the LC-MS that is continuously split to the MS, the fraction collector and the receptor assay. For this step a fraction of the flow is mixed with assay buffer and diluted 100 -fold to reach sub-toxic levels of acetonitrile (ACN) and formic acid (FA). Currently we use flowcells of $150 \mu$ l volume and 300 array spots. To prevent further peak broadening, we typically aim to inject a quantity of sample equivalent to the volume of the flow cell (Chapter 1 ). To replace the flowcell volume every 10 seconds we need to split the LC-MS flow $1 / 20$, resulting in a flow of $9 \mu \mathrm{l} / \mathrm{min}$ to the flowcell. Diluting this $100 \mathrm{x}$, to $900 \mu \mathrm{l} / \mathrm{min}$, the flowcell may be filled with $87 \%$ of a peak in 10 seconds, thus representing a 345 fold dilution of the original extract in 10 seconds.

to allow peak separation within the volume of the flow cell (one peak per volume, see Figure 2). The preliminary results were encouraging, demonstrating that the receptomics assay is compatible with low concentrations $(<0.6 \%)$ of organic solvent in the running buffer. An increased flowrate is also not likely to influence the receptomics assay performance either. A remaining concern may be the effect of a shorter exposure ( $<30$ seconds) on signal strength. Shorter sample exposures have not been tested in this pilot study, but Chapter 1 reports that the response peak maximum of the NK1 receptor was reached within the first 10 seconds of exposure. It is, however, unknown how this translates to other receptor types, and, therefore, the short exposure remains a point of attention. In principle, the fluidic parameter that determines the exposure time is the LC flowrate, so the exposure time could be optimized by tuning the LC setup once a setup is realized.

\section{Envisioned benefits of coupling receptomics to LC-MS}

The extracts from plants, microbes and other organisms represent a huge reservoir of biologically active molecules that have not yet been deeply probed by industry. For the 
current 135 known drugs of plant origin for example, 60.000 plant species have been screened but only using a narrow range of receptors [246-249]. The remaining 300.000 species and the ones screened already could yield many times more novel drugs if the screening would be exhaustive in examining all potential targets in parallel as proposed for this receptomics platform. The screening could involve a pre-screening for bioactivity of the total extract at various dilutions before proceeding to LC-MS coupled receptomics analysis. Direct fluidic coupling of the receptomics assay to acetonitrile containing LC-eluent in the example of Figure 2 can be attempted if bioactivities are found in $\sim 350 x$ diluted extracts. There are many naturally occurring metabolites that have such concentrated bioactivities. A few examples: the glycoside sweeteners in the leaves of Stevia rebaudiana can vary between $4-20 \%$ of dry weight and are up to 250-fold sweeter than sugar [24]. The sweet receptor can sense sweetness from concentrations as low as $4 \mu \mathrm{M}$ stevioside [250], while an average extract may contain up to $4 \mathrm{mM}$. The pungent capsaicinoids found in pepper (Capsicum) fruits have to be diluted 100-1000 fold for a functional bioactivity screen [96, 152]. In a crop like tomato (Solanum lycopersicum), some metabolites like ATP, GABA and Glutamate, are present in 10-50 millimolar ranges [251,252], while the sensitivities of target receptors are in the micro- to nanomolar range $[28,253]$. When bioactive compounds are too low in concentration one can retroactively screen the collected fractions with an autosampler set up. The fractions may then be freeze-dried and re-absorbed in assay buffer at a higher concentrations to remove toxic solvents, similar to the methods in Chapter 3.

\section{Current limitations and opportunities for receptomics}

This section discusses the technical and experimental limitations that currently exist in the application of the receptomics platform for the analysis of various types of samples with various types of receptors and suggest solutions to overcome these limitations. A summary of the key points in this section is given in Table 1.

\section{Calcium buffering and cooperative binding}

In Chapter 5, improvements in receptor sensitivity and signal-to-noise ratio are discussed. Initially, in Chapters 2, 3 and 4, Cameleon YC3.6 was used in all our receptomics arrays because of its high quantum yield, large dynamic range of $>800 \%$ and sensitive $\mathrm{Kd}$ of $\sim 250 \mathrm{nM}$ $[131,204]$. However, two aspects had been overlooked in the initial experimental design: the negative effects of calcium buffering by high sensor concentrations $[207,221]$ and the calcium affinity curves (cooperative binding) of Cameleon YC.6 compared to other sensor types [204, 205]. Both aspects were optimized in Chapter 5 using bitter taste receptors for validation, but within the limitations of the current measurement setup. For example, the Leica stereo fluorescence microscope cannot accurately record fluorescence at expression levels below those used in Chapter 5. Lower sensor concentrations would have further 
lowered the calcium buffering and would have yielded in more sensitive and more accurate measurements. The measurement set-up, therefore, presents some physical limitations. This may be overcome, for example, to some extent by changing to a bioluminescence based setup where probe concentrations can be further reduced while acceptable signal to noise levels are maintained [254].

\section{Membrane display efficiency}

A general issue in receptor screening platforms is the efficiency of cell membrane expression of the receptors. Some GPCR's are notorious for poor cell surface expression and need additional signalling peptides and chaperones to guide the expression in the plasma membrane. One such class of GPCR's with poor cell surface expression are the olfactory receptors which need a signal peptide $[255,256]$ and several accessory proteins to enable functional expression and even then, the levels are still low [257]. There are many factors that can play a role in the trafficking of receptors to and from the cell surface that are still poorly understood. In some cases it could be beneficial to use tissue-specific cell lines instead of a generic cell line like HEK293. In this case, the transfection efficiency of those alternative cell lines needs to be optimized. One of the possible strategies could be the use of lentiviral transfection systems [167].

\section{Response variation}

A general issue in receptor screening platforms is the variable levels of protein expression due to the transient transfection process and differences in cell cycle. Inherent to transient transfection, the plasmid DNA content and transcription levels will vary from cell to cell, leading to a variable expression within one array spot (or within one well of a multi-well plate) but also between array spots and between arrays. The use of a ratiometric calcium sensor partly solves this problem because the calcium measurement is independent of indicator concentration, due to ratiometric imaging. Furthermore, the spot-based model that we introduced in Chapter 6 reduces inter-spot response variation by estimating the spot variation of contrasts of two injections per spot (e.g. sample / blank, or sample-1 / sample-2), rather than the absolute response variation of a single injection.

Next, the receptomics platform strategy of sequential sample injections has an inherent problem of receptor response desensitization [42]. This is caused by receptor signalling termination pathways (e.g. GRK-mediated phosphorylation and $\beta$-arrestin coupling) that lead to receptor internalization. Repeated receptor stimulations will therefore generally lead to a declining response amplitude and -in some instances- absence of a second response. This is shown for example for the CXCR1 and CXCR2 receptors activated by IL8. The desensitization was partly rescued by truncation of the GPCR C-terminal tail which prevents the phosphorylation and subsequent desensitization process [244]. Besides removing the $\mathrm{C}$-terminal phosphorylation sites of the receptor, receptor internalization can also be prevented by silencing the kinases/arrestins or by co-expression of other 
(decoy) receptors that reduce the $\beta$-arrestin recruitment for the target receptor [258, 259]. Another option would be to use the detection of G-protein coupling by miniG proteins and split luciferase [260]. These miniG proteins remain associated with the receptor during the agonist binding and thereby protect the receptor from GRK-mediated phosphorylation.

These solutions, however, require some molecular engineering and have not yet been applied on a larger scale for many different receptors. A more practical strategy to deal with both variation in receptor expression and response saturation is to use a quality control injection (QC) to index the spot responses. The QC sample is a combination of different agonists targeting the receptors displayed on the array. Ideally, a QC sample injection can trigger all receptor types on the array (except for the control spots that lack a recombinant receptor). A specific response to a QC characterizes the degree of functional surface expression in combination with the signalling capacity of that particular receptor of one cell to another. The height of the response may correlate to the relative surface expression/signalling capacity of the receptor within the cells of an array spot. This is shown in Chapter 4 for the expression of the NK1 receptor. Using the QC response height, one could, hypothetically, calibrate each receptor array spot separately for their response capacity towards a standard mix of agonists. By using re-injection of the QC sample several times in a measurement series one may also account for a potential gradual or complete signal loss of saturating responses. Software models can be designed to determine and correct for the response drift due to repeated exposures.

\section{Cell vitality and stress levels}

The success of a receptomics calcium assay depends on the viability and functionality of the cells. Obvious factors like temperature, $\mathrm{pH}$, osmotic or shearing stress are kept under control. But if the cells are stressed by toxic samples, by the receptor or $\mathrm{G}$ protein they express, or by depletion of the energy to pump calcium ions in and out for example, then the measurement of transient calcium dynamics from receptor signalling is impaired. Therefore, the question how long a cell array may last for reliable experiments cannot easily be answered. This depends on the number of exposures, exposure duration and concentration of the agonists, but also the cell surface expression of the receptors and toxicity of the samples etc. Our own experience has shown that, given un-saturating agonist levels, up to 8 sequential agonist doses in a time range of about 1 hour did not reduce the calcium response amplitudes (Chapter 4). Some studies use a cytotoxicity test with ATP or somatostatin to estimate the cell functionality. Somatostatin is particularly appropriate as control in bitter receptor arrays, because in HEK293 cells, the somatostatin receptor couples to the same chimeric $\mathrm{G \alpha}_{16 \mathrm{GUST} 44}$ protein as the bitter taste receptors to report a calcium signal $[209,210]$. In Chapter 6 we have used ATP to mimic a host cell response and we have observed an effect on dosing order with responses declining in time. The use of a functionality control both before and after a measurement series would indicate the level of decrease in cell viability and responsiveness and allow spot specific correction models. 
The use of a control for viability and functionality, like ATP, reports calcium responses that are triggered via similar $G \alpha_{q}-I P_{3}$ signalling pathways. However, the use of these compounds as functionality control may not always be informative because these molecules interact with different host cell receptors and may use alternative pathways or G proteins [261]. Another option to evaluate cell viability may be the implementation of a mitochondrial activity staining using for example, tetramethyl-rhodamine, methyl ester (TMRM), JC-1 or MitoTracker. Mitochondria are the providers of the cellular energy (ATP) in eukaryotic cells through oxidative phosphorylation, during which electrons are transferred from electron donors to electron acceptors such as oxygen. Maintenance of the mitochondrial membrane potential is vital for keeping up ATP production. Mitochondrial membrane potential assays have been developed, even in HTP screening format [262-264]. The use of stains that actively show the mitochondrial membrane potential during a receptomics experiment and that do not interfere with the calcium assay, could also allow us to monitor the viability of the receptor cell arrays.

\section{Monitoring all potential signalling pathways}

Even though GPCR activation can often induce a number of different downstream signalling events by coupling to different $G$ proteins (see Chapter 1) [52], our receptomics assay currently only measures events that generate changes in cytoplasmic calcium [91]. Other signalling pathways involving for example the modulation of cAMP levels or phosphorylation cascades of other proteins (RhoA and ERK1/2) are not monitored. Furthermore, G-proteinindependent signalling, such as $\beta$-arrestin recruitment, are also not detected with the current platform.

For optimal calcium signals, or to steer the response to a calcium second messenger, chimeric G-proteins, like $G \alpha_{16 G U S T 44}$ for taste receptors, are popularly used. These couple the C-terminal receptor binding domain of the $\mathrm{Ga}_{\text {Gustducin }}$ to a $\mathrm{G \alpha}_{15}$ domain which efficiently triggers the $I P_{3}$ pathway $[74,75,265,266]$. G $\alpha_{16 G \text { UST44 }}$ was used for bitter receptor arrays in Chapters 4,5 and 6 by means of a stable expression cell line. In this case, with the use of a recombinant expression system and redirected calcium pathway, any native cell context is missing. This leads to biased agonism; only agonist interactions leading to the introduced functional calcium pathway are measured. Any agonist which recruits other $G$ proteins and elicit a distinct read-out by affecting an alternate or even similar pathway are not detected $[53,267]$. These distinct $G$ proteins and read-outs are a major concern because they may be an important reason for the remaining large number of orphan receptors ( $>20$ years after identifying all members in this family). For example, the short chain free fatty acid receptor FFA2 is activated by propionate through $\mathrm{G \alpha}_{\mathrm{i}}$ and $\mathrm{G} \alpha_{\mathrm{q}} / \mathrm{G} \alpha_{11}$ pathways with distinct physiological effects. This has been confirmed by the finding of an allosteric modulator for FFA2 that enhances the $G \alpha_{i}$ pathway [268]. With our current receptomics platform the $G \alpha_{i}$ activation or modulation would not be measured leading to a considerable "blind spot" for these compounds and their physiological effects. To overcome bias in the detection of 
GPCR activation, the receptomics assay can include receptor spots with probes to measure other secondary messengers like CAMP [269]. The disadvantage of using probes other than calcium probes especially in receptor cell arrays is that the other signalling events usually take much longer to measure. Calcium rise and fall takes seconds to minutes [91], while changes in CAMP and $\beta$-arrestins may take minutes to hours. This makes them less suitable in high throughput screening methods. A solution could be to use the G-protein - receptor interaction itself as a signal for receptor activation. FRET or BRET modified G-protein and receptor systems have been developed to measure receptor and G-protein interaction [270, 271]. However, a more elegant approach may be the use of the recently developed miniG proteins $[260,272]$. These mini- or minimal $\mathrm{G} \alpha$ proteins are engineered GTPase domains of $\mathrm{G} \alpha$ subunits that are left after removal of the membrane anchor, the $\mathrm{G} \beta \gamma$ interaction domain and $\mathrm{N}$ and $\mathrm{C}$-terminal sequences. The remaining miniG protein will not be phosphorylated to its GTP state and as a result it forms a stable interaction with the receptor in its activated or agonist bound state. The miniG proteins are turned into a BRET sensor or a luminescence sensor using a split luciferase fusion to the $\mathrm{N}$-terminus of the miniG and the $\mathrm{C}$-terminus of the receptor. So far, miniG proteins have been made for $\mathrm{G} \alpha_{s^{\prime}}$ $G \alpha_{i}, G \alpha_{q}$ and $G \alpha_{12}$. By studying different receptor-miniG protein combinations in parallel the G-protein subtype biased agonists can be studied without changing the bioluminescent read-out system.

\section{Host cell responses}

As mentioned in the previous section, an advantage of the microfluidic receptomics platform is that it enables sequential injection of samples and determination of differences between samples. But when these samples are complex mixtures, extracts or even food products, there generally are additional cellular calcium responses triggered other than those from specific receptor activations. These endogenous cellular responses or host cell responses may be caused by $\mathrm{pH}$ or salt perturbations in the samples, but usually effects of these parameters can be minimized by adjusting the conditions of the assay. However, samples may also contain compounds that activate a host cell receptor or even contain toxic compounds, and then the assay will measure a host cell response in the mock transfected spots (spots without recombinant receptor). One straightforward solution is to dilute the sample until the host cell response is below threshold. In Chapter 3, a crude chilli pepper extract was measured for bioactivity with the TRPV1 ion channel. At a 300-fold dilution of the extracts, no host cell response was measured anymore in the control cells. Similarly, results with raw pulp of bitter gourd showed specific responses of a bitter taste receptor in a sample of the water phase. The bitter receptor activations were measured at 100-200fold dilutions and in the absence of a host cell response.

However, it is not always possible to dilute a sample sufficiently to dampen the host cell response, without also diluting the compounds of interest too much as well. Chapter 6 shows how a simulated host cell response - in this case a host cell response to ATP- can be 
normalized in a spot-dependent manner using the ATP injections as a reference in a two sample contrast. Bitter receptor spikes were retrieved from the sample comparison even though the host cell response size was variable between spot types. This result shows that a receptor-specific calcium response may be cumulative to a host cell response.

We, therefore, propose to use a host cell response mimic to estimate the host cell response per spot and to use the mimic to normalize host cell responses. This approach was demonstrated using tomato juice samples spiked with bitter compounds (ECRO 2018 conference poster [245]). Tomato is naturally rich in ATP [251] which likely causes the large host cell response of tomato juice samples by activating the nucleotide receptors in HEK293 [253]. In a direct contrast between 5-fold diluted tomato juice with and without a bitter compounds spike and using ATP response to normalize the responses, $50 \%$ of the bitter spikes were recovered. However, the approach of using an ATP host cell response correction only works when it accurately mimics the host cell response which can be determined in a response correlation. Other sources of intracellular calcium modulation, like opening of ion-channels for example, may introduce artefacts.

As long as a calcium read out system is used to detect receptor activation, the host cell response remains something that must always be considered. There is a need for another read-out system that is receptor-specific and not influenced by host cell activation. The earlier mentioned miniG system is such a system. The target receptor is modified with a split luciferase and starts to emit light once fused with the counterpart linked to a miniG protein. This system promises to offer an elegant way of reducing host cell response interference by keeping those responses in the dark.

\section{Auto-fluorescence}

The last limitation that will be discussed is the interference that some samples may cause with the measurement of the fluorescence signal. To monitor any effects on the fluorescence caused by the sample we have introduced a fluorescence control (YC-, Chapters 5 and 6). This is a modified version of YC3.6 where a deletion in the calmodulin and M13 domain makes the protein inactive in sensing calcium. This allows the monitoring of any sample effects on the baseline fluorescence. Samples that display auto-fluorescence at CFP excitation wavelengths usually give signals in both the CFP and YFP emission channels.

When auto-fluorescence occurs in a sample, how can we remove its effects on the calcium signal? One solution would again be to dilute the sample until auto-fluorescence effects are gone, but this will also dilute the sample's bioactivity. Current solutions are sought in computational methods to separate background from actual signal. Using the ratiometric properties of the probe it is possible to separate the auto fluorescence -a signal that increases in both CFP and YFP channels- from a signal that originates from the ratiometric calcium probe - a FRET signal that decreases in the CFP channel and increases in the YFP channel. This method is under development, but is already showing promising results in removing artificial auto-fluorescence from calcium signals (samples spiked with fluorescein) 
and removing auto-fluorescence from natural samples like coffee (unpublished data).

Another solution is based on a reference sample that mimics auto-fluorescence with a compound like fluorescein which does not evoke a calcium response. The auto-fluorescence from this sample could be used to standardize the auto-fluorescence proportional to its relative contribution in each channel. This method is being tested and its effectiveness also looks encouraging.

Another way to work with auto-fluorescent samples is not to use a fluorescence readoud system at all. As mentioned throughout this paragraph, current developments in bioluminescence are very promising and may finally provide the best solution.

Table 1. Troubleshooting receptomics. Current limitations and strategies that may be employed to solve the limitations.

\begin{tabular}{|c|c|c|}
\hline Current limitations & Proposed solutions & References \\
\hline \multicolumn{3}{|l|}{ Receptor cell arrays } \\
\hline current arrays may lack uniformity & $\begin{array}{l}\text { Add transfection agent to the spotmix for } \\
\text { a more uniform transfection }\end{array}$ & [273] \\
\hline $\begin{array}{l}\text { At higher densities spots are at risk of } \\
\text { cross-contamination }\end{array}$ & $\begin{array}{l}\text { Make use of hydrophobic spacing's } \\
\text { between the spots }\end{array}$ & [274] \\
\hline $\begin{array}{l}\text { Large expression differences between } \\
\text { individual cells }\end{array}$ & Synchronize the cell culture & [175] \\
\hline Transfection efficiency of other cell lines & $\begin{array}{l}\text { Switch to virus-based transfection } \\
\text { (Lentivirus) }\end{array}$ & [165] \\
\hline
\end{tabular}

Receptor activation read-out

Calcium buffering by the sensor- the sensor concentration cannot be lowered due to detection limits

Receptor inactivation due to kinases and arrestin recruitment

Calcium and CAMP measurements are too generic and may be induced by host cell receptors as well

Fluorescence measurements pick up auto fluorescence of the matrix

Host cell response of complex samples
Lower sensor levels can be achieved by changing from a fluorescence to a bioluminescence setup

C-terminal kinase activation sites can be removed (truncated),

Kinases/arrestins may be silenced

Co-expression of other (decoy) receptors

(muscarinic receptor)

MiniG proteins protect the activated

receptor state

Detection of G-protein coupling by miniG proteins and split luciferase is receptor specific and will not detect host cell responses

Correct using spotbased axis rotation or indexing using fluorescence standard Use luciferase based probes; CAMP GLOsensor, Calcium BRET Calflux-VTN

Dilute the sample until the host cell response is below detection limit Correlate with a host cell response mimic and eliminate by computation Use receptor specific response readout like miniG proteins
$[260,272]$

Methods are currently being developed, [222]

[105] [260] 


\begin{tabular}{|c|c|c|}
\hline Current limitations & Proposed solutions & References \\
\hline \multicolumn{3}{|l|}{ Imaging } \\
\hline $\begin{array}{l}\text { Simultaneous CFP and YFP emission } \\
\text { recordings - to eliminate time difference } \\
\text { between recordings }\end{array}$ & $\begin{array}{l}\text { Use light path with beam splitters and } \\
\text { dual read-out on two camera's or a split } \\
\text { camera } \\
\text { Switch to measuring a single wavelength }\end{array}$ & [91] \\
\hline Noise levels & $\begin{array}{l}\text { Use a stable light source } \\
\text { Use other synthetic probes like Fluo- } 4 \text { in } \\
\text { combination with RFP to obtain better } \\
\text { signals } \\
\text { Use more sensitive cameras. Eg cooled } \\
\text { camera (EmCCD or CMOS) }\end{array}$ & [91] \\
\hline Long working distance causes signal loss & $\begin{array}{l}\text { Cell array with a mini camera on top of a } \\
\text { sensor } \\
\text { Creating a lens-free system with an array } \\
\text { directly on top of a sensor chip }\end{array}$ & \\
\hline \multicolumn{3}{|l|}{ Data processing } \\
\hline Background auto fluorescence elimination & $\begin{array}{l}\text { Implementation of data processing } \\
\text { module to remove background } \\
\text { fluorescence }\end{array}$ & $\begin{array}{l}\text { Methods are } \\
\text { currently } \\
\text { being } \\
\text { developed }\end{array}$ \\
\hline $\begin{array}{l}\text { Spot based processing to pixel based; a } \\
\text { spot contains } \sim 25 \text { cells that are averaged }\end{array}$ & $\begin{array}{l}\text { Pixel based processing gives information } \\
\text { on signal distribution within a spot. }\end{array}$ & \\
\hline
\end{tabular}

\section{Receptomics: towards a plug-and-play platform}

Downsizing technology, miniaturization and the development of hand-held devices have led to a break-through in sensitivity, throughput and cost savings in sensor technology and have led to new application areas $[275,276]$. Also for biosensors, if we can downscale the biological components, such as using a cell array of $1 \mathrm{~cm}^{2}$ instead of a multi-well plate of $\sim 50 \mathrm{~cm}^{2}$, we may potentially reach higher sensitivity, higher throughput and save costs [94]. Current receptomics cell arrays contain about 300 spots composed of 25-50 cells each. The cell layer that covers a surface of about $1 \mathrm{~cm}^{2}$ contains over 1 million cells. The optical detection converts one cell into approximately one pixel (Chapter 4) making one pixel currently the smallest detection unit.

The receptomics platform has been developed with the aim in mind of non-targeted analysis of the biological function of compounds in extracts and for potentially downscaling the technology and even developing hand-held functional receptor screening assays. It was soon realized that in order to measure a $1 \mathrm{~cm}^{2}$ cell array expressing hundreds of different receptors, a different microscopy platform was needed. In Chapters 2 and 3, the Zeiss 510META confocal laser scanning microscope was used to image the calcium responses from stable receptor expressing cell lines. However, even at the lowest possible magnification, this microscope could only image $1 \mathrm{~mm}^{2}$ equivalent to maximum 9 cell array spots at the time ( $3 \times 3$ array). A larger scanning area was needed. The BD Pathway Imager system with 
its moving objective, was able to make sequential scans of the flowcell area, but the scan speed of 30-60 seconds for $1 \mathrm{~cm}^{2}$ was not fast enough to accurately capture the calcium dynamics of each spot across the array. The Leica stereo fluorescence microscope (M205 FA) did suffice but with a loss of resolution ( 1 pixel/cell). It met our criteria for acquisition speed ( 3 seconds/frame), dual read-out (CFP and YFP emission), and large scanning field $\left(\sim 1 \mathrm{~cm}^{2}\right)$. However, the disadvantages of this imaging platform were the longer working distance (loss of signal or increase of noise), the filter-based excitation and emission (allows bleed through of CFP emission light) and an unstable light source. Solutions to some shortcomings could be realised via the receptomics software. While the original purpose of the software was to sort and combine the data parameters, it was soon extended with an interpolation algorithm (smoothing) to compensate for the timing difference of the CFP and YFP images and to reduce the noise levels. This interpolation of the time difference between the images was necessary for an accurate YFP/CFP ratio of the FRET sensor. A simple marker, placed outside the flowcell, but in the field of view, was able to capture any fluctuation of the light source and allowed for auto correction of the data. Finally the mixed-model for sample comparisons was added the software (used in Chapters 4, 5 and 6).

The receptomics platform is not yet a plug-and-play platform and still needs trained personnel to culture the cells, prepare cell arrays, operate the fluidic system and fluorescence microscope. To perform a comprehensive analysis of the experimental outcome, one needs an understanding of the signalling pathways and design the experiments accordingly. If we want to continue developing the receptomics technology and eventually reach the goal of becoming a high content screening technology with applications as described in more detail in the next section, the platform will need to continue to evolve. Table 1 gives several suggestions for further development of the receptomics technology.

\section{Tailor made receptomics arrays}

Receptomics technology has the potential to become a versatile platform because it combines low costs with obtaining functional information on ligand-receptor interactions, generating novel applications. It allows assaying of a sample against endless variations and combinations of printed receptors, G-proteins, sensors, co-factors etc. in a flow context with a wide range of possibilities for pre- and post-measurement calibration.

The versatility extends to the use of different cell types. Even though the reverse transfection is best performed with an "easy" cell line like HEK293 cells, the use of other cell lines have already been reported for reverse transfection [273]. Using different cell types, different transfection approaches may be necessary like virus-based transfection systems [165]. An important consideration in the choice of cell type is the profile of native expressed receptors and signalling molecules. Studies show that these may vary [73], 
and hence have important implications for host cell response to samples. On the other hand, there are studies that utilize the unique receptor repertoires of specialized cells for functional screenings. Studies show that exposure of bitter compounds to gastric parietal cells trigger the secretion of gastric acid [277, 278]. Even without knowing which bitter receptors are involved, researchers were able to study bitter modulating compounds based on the increase or reduction of gastric acid secretion. Though no receptor-compound interactions were measured directly, these findings did give important leads to discovering new bitter modulating compounds. Our platform could be used to transfect these cells with siRNA silencing or CRISPR-Cas knock outs of such specialized cells. In this way, the direct receptor-compound relations could still be shown. Similarly, dCas9-VPR like systems could be used for (inducible) upregulation of genes without the need to clone them [279].

\section{Application areas for receptomics}

\section{Pharmaceutical and medical applications}

If a low cost receptomics chip could be produced that, for example, involves all human receptors in combination with all G proteins ( 800 GPCR-s $\times 30$ G-proteins combinations), with a suitable detection system (miniG bioluminescence system) this would offer unprecedented research opportunities to discover novel drugs and investigate modes of action. If this could then be expanded with all SNP variants it could also become a significant tool to understand genetic causes underlying human diseases.

However, one of the many hurdles, as mentioned in the previous section, is that GPCR's may employ different signalling pathways depending on their cellular context. Activation of a receptor may lead to different physiological effects depending on the 'wiring' of the receptor. An odorant receptor expressed in the epithelium of the nasal cavity has a different functionality and signalling pathway than an odorant receptor expressed on spermatozoa [280]. But even without understanding the cellular context, the knowledge that may be obtained by a receptomics wide screen of potential drug targets will at least provide leads for further, tissue specific, studies. Since about $40 \%$ of today's drugs target a GPCR [8688], the development of tools to further improve the specificity and effectiveness of these drugs is highly justified.

About 140 GPCR's are still called orphans [1, 107], meaning that their function(s) and endogenous activating ligand(s) have not yet been identified. Receptomics approaches could tap into this huge unknown and contribute to finding novel targets for drug discovery due to its potential to lower costs for the screening of huge libraries of all potentially relevant combinations of factors (receptors vs. G protein vs. co-factor x vs. ..) and screen complex biological samples [87]. This "reverse pharmacology" strategy uses an orphan receptor as bait to find novel interactions with target compound libraries [240]. 


\section{Flavour: Taste and olfaction}

The off-taste of a drug may be experienced as side-effects. A bitter taste can have serious consequences on the palatability and the acceptance of the drug by patients. Especially in paediatric medicines, tablets or oral liquids may be too bitter to swallow which makes acceptance of paediatric medicinal products of great importance to patient recovery [281]. Many active pharmaceutical ingredients have an adverse bitter taste including chloropheniramine and diphenhydramine, both potent antihistamines that work well against allergic reactions. Other examples of drugs that can activate $T 2 R s$ include antimalarial drugs like quinine and chloroquine, and antibiotics like erythromycin and ofloxacin.

Masking may be an option and there are different routes to achieve masking of the bitter taste [282]. An alternative can be modulating bitter taste at a molecular level by creating a "molecular barrier" or coating for the medicinal compounds obscuring of the taste, or at the receptor level by using sweeteners and other flavouring agents as masking agents or by removing the bitter taste by blocking the relevant bitter receptors. For many years flavourresearchers have been looking for bitter taste modulators using bitter taste receptor functional assays in order to find blockers or masks which can reduce some of the bitterness of medicines [283, 284]. Besides established multi-well techniques to characterize bitter taste modulators, the receptomics assay may be a helpful tool to detect the modulation of a dynamic response for all bitter receptors (and SNP variants) simultaneously.

Aroma and flavour are key determinants in acceptance and hence preference, of consumers for particular (new) products. However, often the bioactive molecules responsible for a certain sensory experience have remained unknown. Considering that we are now in the era of low sugar and low salt trends, both with known flavour enhancers, it has never been more important to discover which molecular ligands present in different foodstuffs play the most dominant role in our aroma and taste experience. For taste and flavour research the most promising feature of the receptomics platform is the possibility of screening and comparing complex samples (matrices). In product development or crop breeding, subtle sample differences in taste are difficult to measure using taste panels where subjectivity can be an issue. If a receptomics platform could provide a means of pre-screening samples / varieties, particularly for the presence of off-flavours, this could become a valuable tool. Preliminary results have indicated that indeed the receptomics platform is able to measure vegetable sample differences despite the complexity of the samples (host cell responses and or auto-fluorescence, unpublished data). Before the receptomics system is up to such task, interpretation algorithms may need to be developed to link receptor activation profiles to a taste perception.

\section{Technology roadmap}

A way to visualise the possible developments for a new technology is by means of a technology road map as shown below in Figure 3. 


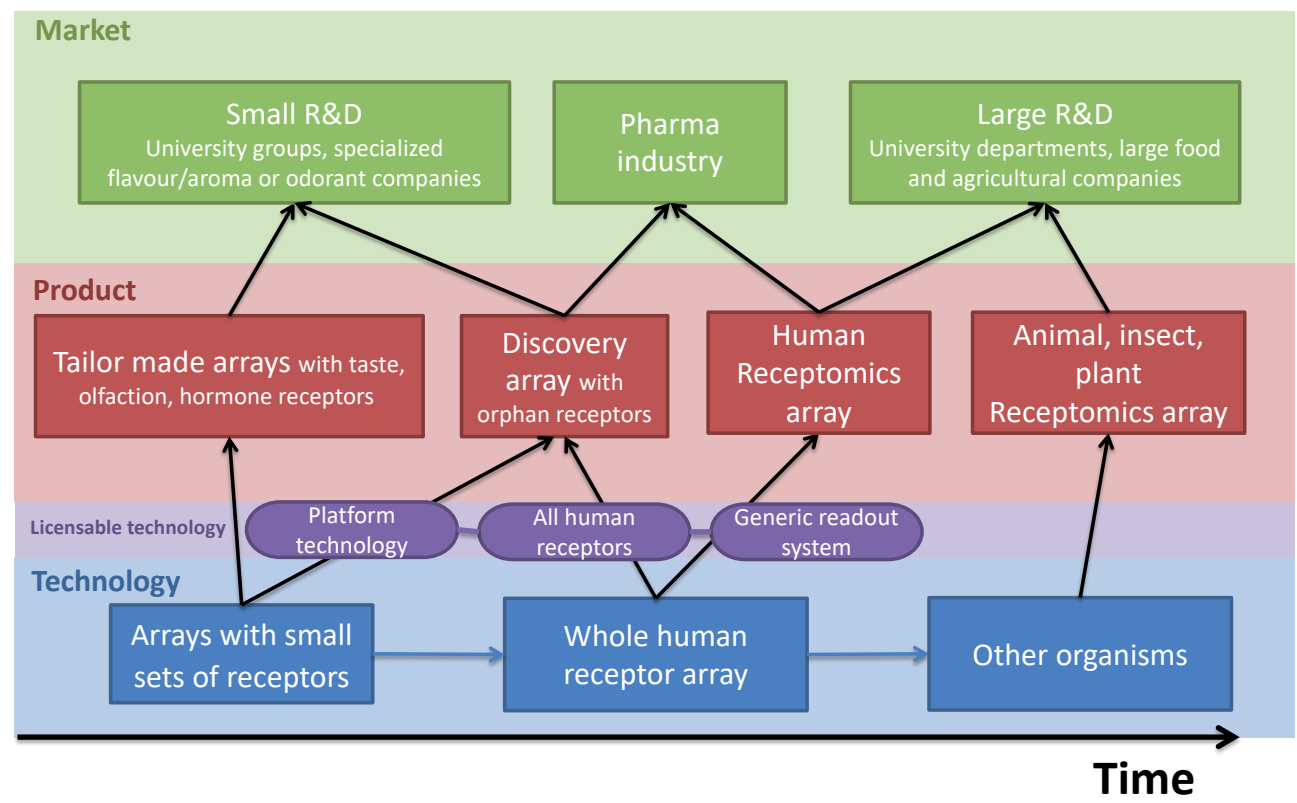

Figure 3, A technological roadmap for receptomics. The envisioned timeline is shown from left to right. From bottom to top the evolution of technology into products and markets is given.

\section{Societal impact}

After the technological and scientific aspects of the receptomics technology have been discussed above, some final words on the potential social impact of this technology. As this technology brings us a step closer to realizing a nose-on-a-chip or a tongue-on-a-chip, taste and smell may no longer be exclusive qualities of the consumer [285] as the technology aims to replicate these senses [286, 287]. Nowadays, flavour and fragrance companies rely heavily on the expertise of their taste panels and even on single individuals (the 'Nose'). With the introduction of devices that can measure taste or smell, these panels may be partially replaced, especially in the early phases of R\&D, when technology is able to properly emulate the human senses. Theoretically, a taste and smell sensor can predict the overall palatability of a product better because we could also incorporate the impact of genetic variation and rule out environmental/societal/cultural differences that lie at the base of differences in individual taste experiences [110]. So a single receptomics chip might come to represent receptors of many different panellists. But - can all taste panels be replaced? This doesn't seem te be very likely, because taste perception is not merely the sum of the receptor responses. Even if the technology is able to measure all aspects of the signal transduction there are parameters that an artificial taste sensor is not able to measure. Texture, colour and ambiance play a hedonic role in food choice and consumption [285]. They determine, together with flavour, the palatability of the food. 
Our western society is more and more based on individualism. We all want maximum choice and to be offered a wide range of products in the supermarket. We all make our own choices and every person is unique. The idea that the uniqueness of individuals can be measured by generic chips for taste and smell might, to some, be a bit disturbing. And we may even find ourselves outsmarted by these electronic tongues and noses in the future. But the technology of the future will also embrace the uniqueness of humans with a tailor-made receptomics array that carries your unique genetic make-up.

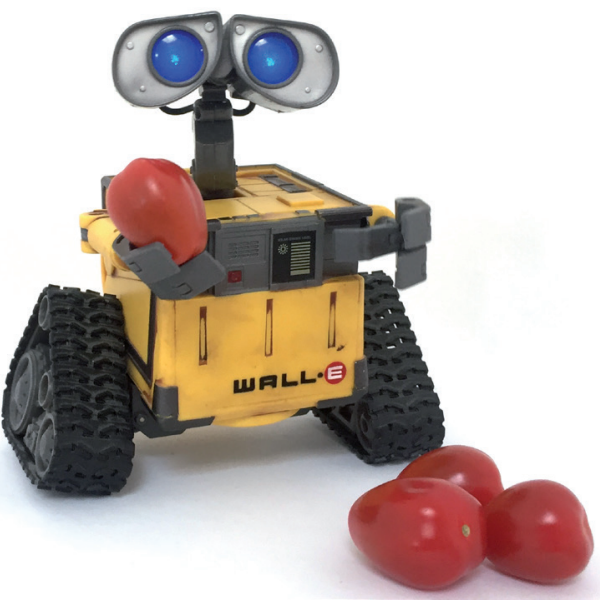

Figure 4, The electronic nose and tongue technology may be difficult to comprehend and cause naïve associations in society.

Photo by Margriet Roelse

Since individual sequencing projects are increasing in popularity - companies like 23andme (www.23andme.com) advertise that you can "meet your genes". Medical treatments will become more personalized to fit your unique biochemical make-up. A receptomics chip that allows the display of your unique set of receptors could play a role in the field of personalized foods and medicines [288-291] so that you may "meet your receptors" as well. 

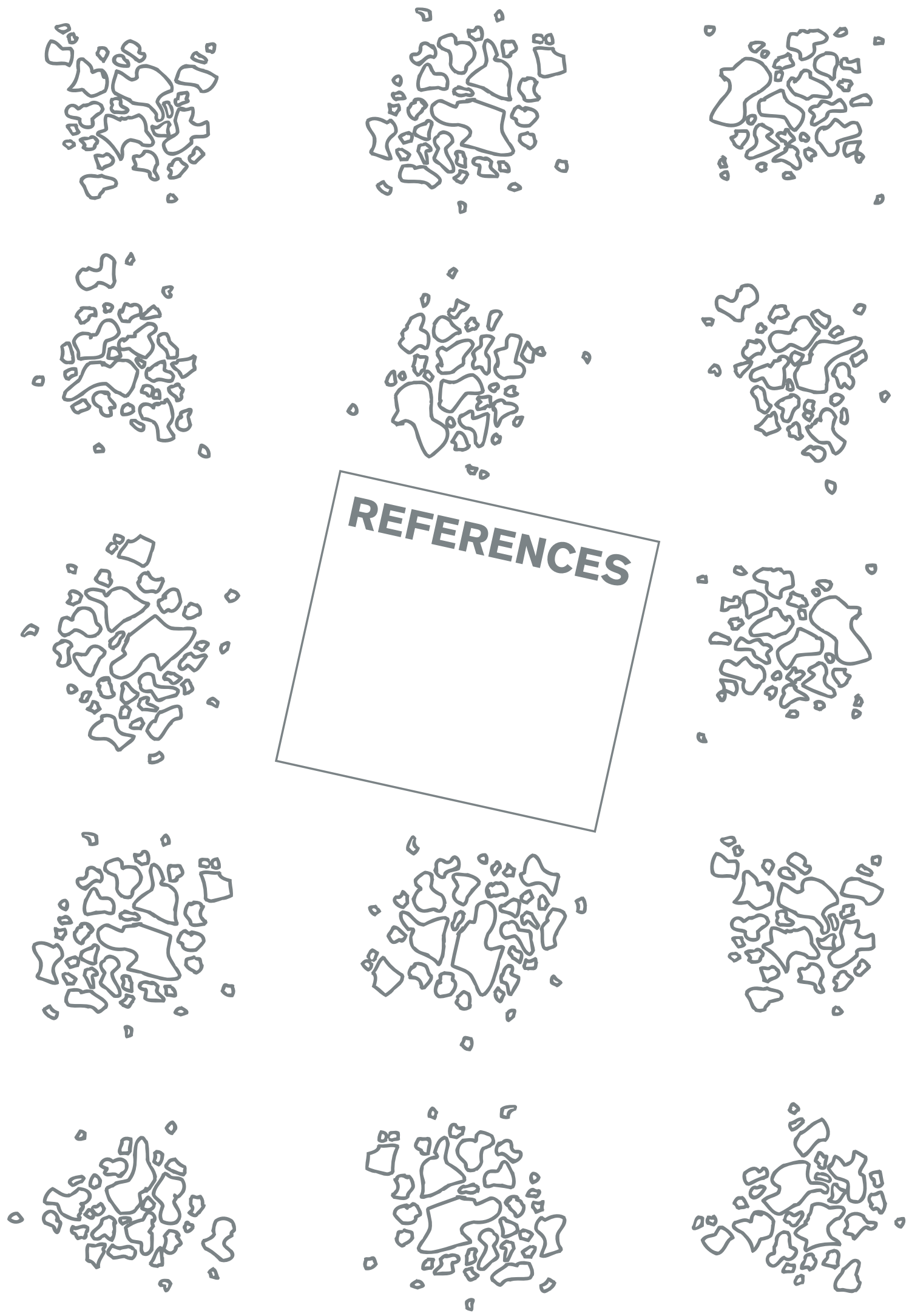
[1] T.K. Bjarnadottir, D.E. Gloriam, S.H. Hellstrand, H. Kristiansson, R. Fredriksson, H.B. Schioth, Comprehensive repertoire and phylogenetic analysis of the $\mathrm{G}$ protein-coupled receptors in human and mouse, Genomics, 88 (2006) 263-273.

[2] T.K. Attwood, J.B. Findlay, Fingerprinting G-protein-coupled receptors, Protein Eng, 7 (1994) 195203.

[3] S.M. Foord, T.I. Bonner, R.R. Neubig, E.M. Rosser, J.P. Pin, A.P. Davenport, M. Spedding, A.J. Harmar, International Union of Pharmacology. XLVI. G protein-coupled receptor list, Pharmacol Rev, 57 (2005) 279-288.

[4] H.A. Watkins, M. Au, D.L. Hay, The structure of secretin family GPCR peptide ligands: implications for receptor pharmacology and drug development, Drug discovery today, 17 (2012) 1006-1014.

[5] L. Chun, W.H. Zhang, J.F. Liu, Structure and ligand recognition of class C GPCRs, Acta Pharmacol Sin, 33 (2012) 312-323.

[6] C.G. Alvaro, J. Thorner, Heterotrimeric G Protein-coupled Receptor Signaling in Yeast Mating Pheromone Response, The Journal of biological chemistry, 291 (2016) 7788-7795.

[7] P.S. Klein, T.J. Sun, C.L. Saxe, A.R. Kimmel, R.L. Johnson, P.N. Devreotes, A chemoattractant receptor controls development in Dictyostelium discoideum, Science, 241 (1988) 1467-1472.

[8] H.C. Huang, P.S. Klein, The Frizzled family: receptors for multiple signal transduction pathways, Genome Biol, 5 (2004) 234.

[9] Y. Chen, G. Struhl, In vivo evidence that Patched and Smoothened constitute distinct binding and transducing components of a Hedgehog receptor complex, Development, 125 (1998) 4943-4948.

[10] A. de Mendoza, A. Sebe-Pedros, I. Ruiz-Trillo, The evolution of the GPCR signaling system in eukaryotes: modularity, conservation, and the transition to metazoan multicellularity, Genome Biol Evol, 6 (2014) 606-619.

[11] K.L. Pierce, R.T. Premont, R.J. Lefkowitz, Seven-transmembrane receptors, Nature reviews. Molecular cell biology, 3 (2002) 639-650.

[12] A.J. Kooistra, C. de Graaf, H. Timmerman, The Receptor Concept in 3D: From Hypothesis and Metaphor to GPCR-Ligand Structures, Neurochem Res, 39 (2014) 1850-1861.

[13] G.L. Collingridge, R.W. Olsen, J. Peters, M. Spedding, A nomenclature for ligand-gated ion channels, Neuropharmacology, 56 (2009) 2-5.

[14] H.Y. Li, TRP Channel Classification, Adv Exp Med Biol, 976 (2017) 1-8.

[15] J. Krusek, I. Dittert, T. Hendrych, P. Hnik, M. Horak, M. Petrovic, M. Sedlacek, K. Susankova, L. Svobodova, K. Tousova, E. Ujec, V. Vlachova, L. Vyklicky, F. Vyskocil, L. Vyklicky, Jr., Activation and modulation of ligand-gated ion channels, Physiol Res, 53 Suppl 1 (2004) S103-113.

[16] P. Lu, C.H. Zhang, L.M. Lifshitz, R. ZhuGe, Extraoral bitter taste receptors in health and disease, The Journal of general physiology, 149 (2017) 181-197.

[17] J. Chandrashekar, M.A. Hoon, N.J. Ryba, C.S. Zuker, The receptors and cells for mammalian taste, Nature, 444 (2006) 288-294.

[18] C.A. Running, B.A. Craig, R.D. Mattes, Oleogustus: The Unique Taste of Fat, Chemical senses, 40 (2015) 507-516.

[19] M.M. Galindo, N. Voigt, J. Stein, J. van Lengerich, J.D. Raguse, T. Hofmann, W. Meyerhof, M. Behrens, G Protein-Coupled Receptors in Human Fat Taste Perception, Chemical senses, 37 (2012) 123-139.

[20] A.L. Huang, X. Chen, M.A. Hoon, J. Chandrashekar, W. Guo, D. Trankner, N.J. Ryba, C.S. Zuker, The cells and logic for mammalian sour taste detection, Nature, 442 (2006) 934-938.

[21] Y. Ishimaru, Molecular mechanisms underlying the reception and transmission of sour taste information, Biosci Biotech Bioch, 79 (2015) 171-176.

[22] S. Roper, The taste of table salt, Pflug Arch Eur J Phy, 467 (2015) 457-463.

[23] X. Li, L. Staszewski, H. Xu, K. Durick, M. Zoller, E. Adler, Human receptors for sweet and umami taste, Proceedings of the National Academy of Sciences of the United States of America, 99 (2002) 46924696.

[24] M. Behrens, W. Meyerhof, C. Hellfritsch, T. Hofmann, Sweet and Umami Taste: Natural Products, Their Chemosensory Targets, and Beyond, Angew Chem Int Edit, 50 (2011) 2220-2242.

[25] S.K. Kim, Y. Chen, R. Abrol, W.A. Goddard, 3rd, B. Guthrie, Activation mechanism of the G proteincoupled sweet receptor heterodimer with sweeteners and allosteric agonists, Proceedings of the National Academy of Sciences of the United States of America, 114 (2017) 2568-2573. 
[26] Y. Ohtsu, Y. Nakagawa, M. Nagasawa, S. Takeda, H. Arakawa, I. Kojima, Diverse signaling systems activated by the sweet taste receptor in human GLP-1-secreting cells, Mol Cell Endocrinol, 394 (2014) 70-79.

[27] H. Xu, L. Staszewski, H.X. Tang, E. Adler, M. Zoller, X.D. Li, Different functional roles of T1R subunits in the heterorneric taste receptors, Proceedings of the National Academy of Sciences of the United States of America, 101 (2004) 14258-14263.

[28] F. Zhang, B. Klebansky, R.M. Fine, H. Xu, A. Pronin, H. Liu, C. Tachdjian, X. Li, Molecular mechanism for the umami taste synergism, Proceedings of the National Academy of Sciences of the United States of America, 105 (2008) 20930-20934.

[29] F. Zhang, B. Klebansky, R.M. Fine, H. Liu, H. Xu, G. Servant, M. Zoller, C. Tachdjian, X. Li, Molecular mechanism of the sweet taste enhancers, Proceedings of the National Academy of Sciences of the United States of America, 107 (2010) 4752-4757.

[30] G. Servant, C. Tachdjian, X.Q. Tang, S. Werner, F. Zhang, X. Li, P. Kamdar, G. Petrovic, T. Ditschun, A. Java, P. Brust, N. Brune, G.E. DuBois, M. Zoller, D.S. Karanewsky, Positive allosteric modulators of the human sweet taste receptor enhance sweet taste, Proceedings of the National Academy of Sciences of the United States of America, 107 (2010) 4746-4751.

[31] T. Yasuo, Y. Kusuhara, K. Yasumatsu, Y. Ninomiya, Multiple receptor systems for glutamate detection in the taste organ, Biol Pharm Bull, 31 (2008) 1833-1837.

[32] J. Chandrashekar, K.L. Mueller, M.A. Hoon, E. Adler, L. Feng, W. Guo, C.S. Zuker, N.J. Ryba, T2Rs function as bitter taste receptors, Cell, 100 (2000) 703-711.

[33] W. Meyerhof, C. Batram, C. Kuhn, A. Brockhoff, E. Chudoba, B. Bufe, G. Appendino, M. Behrens, The molecular receptive ranges of human TAS2R bitter taste receptors, Chemical senses, 35 (2010) 157170.

[34] M. Behrens, W. Meyerhof, Bitter taste receptors and human bitter taste perception, Cell Mol Life Sci, 63 (2006) 1501-1509.

[35] E. Rozengurt, C. Sternini, Taste receptor signaling in the mammalian gut, Curr Opin Pharmacol, 7 (2007) 557-562.

[36] N. Singh, M. Vrontakis, F. Parkinson, P. Chelikani, Functional bitter taste receptors are expressed in brain cells, Biochem Biophys Res Commun, 406 (2011) 146-151.

[37] K.I. Liszt, J.P. Ley, B. Lieder, M. Behrens, V. Stoger, A. Reiner, C.M. Hochkogler, E. Kock, A. Marchiori, J. Hans, S. Widder, G. Krammer, G.J. Sanger, M.M. Somoza, W. Meyerhof, V. Somoza, Caffeine induces gastric acid secretion via bitter taste signaling in gastric parietal cells, Proceedings of the National Academy of Sciences of the United States of America, 114 (2017) E6260-E6269.

[38] S.P. Shirazi-Beechey, K. Daly, M. Al-Rammahi, A.W. Moran, D. Bravo, Role of nutrient-sensing taste 1 receptor (T1R) family members in gastrointestinal chemosensing, Br J Nutr, 111 Suppl 1 (2014) S815.

[39] S.B. Liggett, Bitter taste receptors on airway smooth muscle as targets for novel bronchodilators, Expert Opin Ther Tar, 17 (2013) 721-731.

[40] H.M. Lee, Y. Kim, Drug Repurposing Is a New Opportunity for Developing Drugs against Neuropsychiatric Disorders, Schizophrenia research and treatment, 2016 (2016) 6378137.

[41] S.B. Gacasan, D.L. Baker, A.L. Parrill, G protein-coupled receptors: the evolution of structural insight, AIMS Biophys, 4 (2017) 491-527.

[42] W. Yang, S.H. Xia, Mechanisms of regulation and function of G-protein-coupled receptor kinases, World J Gastroentero, 12 (2006) 7753-7757.

[43] C.D. Hanlon, D.J. Andrew, Outside-in signaling--a brief review of GPCR signaling with a focus on the Drosophila GPCR family, J Cell Sci, 128 (2015) 3533-3542.

[44] N.M. Duc, H.R. Kim, K.Y. Chung, Recent Progress in Understanding the Conformational Mechanism of Heterotrimeric G Protein Activation, Biomol Ther (Seoul), 25 (2017) 4-11.

[45] V.V. Gurevich, E.V. Gurevich, The structural basis of the arrestin binding to GPCRs, Mol Cell Endocrinol, 484 (2019) 34-41.

[46] S. Urwyler, Allosteric modulation of family C G-protein-coupled receptors: from molecular insights to therapeutic perspectives, Pharmacol Rev, 63 (2011) 59-126.

[47] E.F. Grady, A.M. Garland, P.D. Gamp, M. Lovett, D.G. Payan, N.W. Bunnett, Delineation of the endocytic pathway of substance $P$ and its seven-transmembrane domain NK1 receptor, Molecular biology of the cell, 6 (1995) 509-524. 
[48] P. Seeman, Cannabidiol is a partial agonist at dopamine D2High receptors, predicting its antipsychotic clinical dose, Transl Psychiat, 6 (2016).

[49] K.J. Brown, A.S. Laun, Z.H. Song, Cannabidiol, a novel inverse agonist for GPR12, Biochem Biophys Res Commun, 493 (2017) 451-454.

[50] R.M. Snider, J.W. Constantine, J.A. Lowe, 3rd, K.P. Longo, W.S. Lebel, H.A. Woody, S.E. Drozda, M.C. Desai, F.J. Vinick, R.W. Spencer, et al., A potent nonpeptide antagonist of the substance P (NK1) receptor, Science, 251 (1991) 435-437.

[51] N.A. Guzzetta, Phenoxybenzamine in the treatment of hypoplastic left heart syndrome: a core review, Anesth Analg, 105 (2007) 312-315.

[52] Y. Liu, Y. Yang, R. Ward, S. An, X.X. Guo, W. Li, T.R. Xu, Biased signalling: the instinctive skill of the cell in the selection of appropriate signalling pathways, The Biochemical journal, 470 (2015) 155-167.

[53] J.S. Smith, R.J. Lefkowitz, S. Rajagopal, Biased signalling: from simple switches to allosteric microprocessors, Nature reviews. Drug discovery, 17 (2018) 243-260.

[54] G.T. Wong, K.S. Gannon, R.F. Margolskee, Transduction of bitter and sweet taste by gustducin, Nature, 381 (1996) 796-800.

[55] M. Ozeck, P. Brust, H. Xu, G. Servant, Receptors for bitter, sweet and umami taste couple to inhibitory G protein signaling pathways, European journal of pharmacology, 489 (2004) 139-149.

[56] A. Caicedo, E. Pereira, R.F. Margolskee, S.D. Roper, Role of the G-protein subunit alpha-gustducin in taste cell responses to bitter stimuli, Journal of Neuroscience, 23 (2003) 9947-9952.

[57] A. Tromelin, Odour perception: A review of an intricate signalling pathway, Flavour Frag J, 31 (2016) 107-119.

[58] M. Arimont, S.L. Sun, R. Leurs, M. Smit, I.J.P. de Esch, C. de Graaf, Structural Analysis of Chemokine Receptor-Ligand Interactions, J Med Chem, 60 (2017) 4735-4779.

[59] K. Zavala, M.W. Vandewege, F.G. Hoffmann, J.C. Opazo, Evolution of the beta-adrenoreceptors in vertebrates, Gen Comp Endocrinol, 240 (2017) 129-137.

[60] H.A. Cespedes, K. Zavala, M.W. Vandewege, J.C. Opazo, Evolution of the alpha2-adrenoreceptors in vertebrates: ADRA2D is absent in mammals and crocodiles, Gen Comp Endocrinol, 250 (2017) 8594.

[61] J.D. McCorvy, B.L. Roth, Structure and function of serotonin G protein-coupled receptors, Pharmacol Ther, 150 (2015) 129-142.

[62] I. Obara, V. Telezhkin, I. Alrashdi, P.L. Chazot, Histamine, histamine receptors, and neuropathic pain relief, Br J Pharmacol, (2019).

[63] J.M. Beaulieu, R.R. Gainetdinov, The physiology, signaling, and pharmacology of dopamine receptors, Pharmacol Rev, 63 (2011) 182-217.

[64] A.I. Spielman, Gustducin and its role in taste, J Dent Res, 77 (1998) 539-544.

[65] S.K. Mclaughlin, P.J. Mckinnon, R.F. Margolskee, Gustducin Is a Taste-Cell-Specific G-Protein Closely Related to the Transducins, Nature, 357 (1992) 563-569.

[66] R.F. Margolskee, Molecular mechanisms of bitter and sweet taste transduction, The Journal of biological chemistry, 277 (2002) 1-4.

[67] J. Meshki, S.D. Douglas, J.P. Lai, L. Schwartz, L.E. Kilpatrick, F. Tuluc, Neurokinin 1 receptor mediates membrane blebbing in HEK293 cells through a Rho/Rho-associated coiled-coil kinase-dependent mechanism, The Journal of biological chemistry, 284 (2009) 9280-9289.

[68] F. Giannone, G. Malpeli, V. Lisi, S. Grasso, P. Shukla, D. Ramarli, S. Sartoris, V. Monsurro, M. Krampera, E. Amato, G. Tridente, M. Colombatti, M. Parenti, G. Innamorati, The puzzling uniqueness of the heterotrimeric G15 protein and its potential beyond hematopoiesis, J Mol Endocrinol, 44 (2010) 259-269.

[69] M.P. Strathmann, M.I. Simon, G alpha 12 and $G$ alpha 13 subunits define a fourth class of $G$ protein alpha subunits, Proceedings of the National Academy of Sciences of the United States of America, 88 (1991) 5582-5586.

[70] P. Mombaerts, Genes and ligands for odorant, vomeronasal and taste receptors, Nat Rev Neurosci, 5 (2004) 263-278.

[71] D. Kamato, P. Mitra, F. Davis, N. Osman, R. Chaplin, P.J. Cabot, R. Afroz, W. Thomas, W. Zheng, H. Kaur, M. Brimble, P.J. Little, Gaq proteins: molecular pharmacology and therapeutic potential, Cell Mol Life Sci, 74 (2017) 1379-1390. 
[72] R.M. Eglen, A. Gilchrist, T. Reisine, The use of immortalized cell lines in GPCR screening: The good, bad and ugly, Combinatorial chemistry \& high throughput screening, 11 (2008) 560-565.

[73] B.K. Atwood, J. Lopez, J. Wager-Miller, K. Mackie, A. Straiker, Expression of G protein-coupled receptors and related proteins in HEK293, AtT20, BV2, and N18 cell lines as revealed by microarray analysis, BMC genomics, 12 (2011) 14.

[74] S. Offermanns, M.I. Simon, G alpha 15 and G alpha 16 couple a wide variety of receptors to phospholipase C, The Journal of biological chemistry, 270 (1995) 15175-15180.

[75] T. Ueda, S. Ugawa, H. Yamamura, Y. Imaizumi, S. Shimada, Functional interaction between T2R taste receptors and G-protein alpha subunits expressed in taste receptor cells, The Journal of neuroscience: the official journal of the Society for Neuroscience, 23 (2003) 7376-7380.

[76] H. Zhuang, H. Matsunami, Synergism of accessory factors in functional expression of mammalian odorant receptors, The Journal of biological chemistry, 282 (2007) 15284-15293.

[77] Z. Zhang, Z. Zhao, R. Margolskee, E. Liman, The transduction channel TRPM5 is gated by intracellular calcium in taste cells, The Journal of neuroscience : the official journal of the Society for Neuroscience, 27 (2007) 5777-5786.

[78] J. Ziauddin, D.M. Sabatini, Microarrays of cells expressing defined cDNAs, Nature, 411 (2001) 107110.

[79] E. Palmer, Cell-based microarrays: overview, Methods in molecular biology, 706 (2011) 1-12.

[80] J.K. Rantala, R. Makela, A.R. Aaltola, P. Laasola, J.P. Mpindi, M. Nees, P. Saviranta, O. Kallioniemi, A cell spot microarray method for production of high density siRNA transfection microarrays, BMC genomics, 12 (2011) 162.

[81] Y.M. Mishina, C.J. Wilson, L. Bruett, J.J. Smith, C. Stoop-Myer, S. Jong, L.P. Amaral, R. Pedersen, S.K. Lyman, V.E. Myer, B.L. Kreider, C.M. Thompson, Multiplex GPCR assay in reverse transfection cell microarrays, Journal of biomolecular screening, 9 (2004) 196-207.

[82] S. Fujita, R. Onuki-Nagasaki, J. Fukuda, J. Enomoto, S. Yamaguchi, M. Miyake, Development of superdense transfected cell microarrays generated by piezoelectric inkjet printing, Lab on a chip, 13 (2013) 77-80.

[83] Z.T. Yu, K. Kamei, H. Takahashi, C.J. Shu, X. Wang, G.W. He, R. Silverman, C.G. Radu, O.N. Witte, K.B. Lee, H.R. Tseng, Integrated microfluidic devices for combinatorial cell-based assays, Biomedical microdevices, 11 (2009) 547-555.

[84] K. Woodruff, S.J. Maerkl, A High-Throughput Microfluidic Platform for Mammalian Cell Transfection and Culturing, Scientific reports, 6 (2016) 23937.

[85] E.H. Oh, S.H. Lee, S.H. Lee, H.J. Ko, T.H. Park, Cell-based high-throughput odorant screening system through visualization on a microwell array, Biosensors \& bioelectronics, 53 (2014) 18-25.

[86] R.M. Eglen, Functional G protein-coupled receptor assays for primary and secondary screening, Combinatorial chemistry \& high throughput screening, 8 (2005) 311-318.

[87] S. Wilson, D. Bergsma, Orphan G-protein coupled receptors: novel drug targets for the pharmaceutical industry, Drug design and discovery, 17 (2000) 105-114.

[88] A.S. Hauser, M.M. Attwood, M. Rask-Andersen, H.B. Schioth, D.E. Gloriam, Trends in GPCR drug discovery: new agents, targets and indications, Nature reviews. Drug discovery, 16 (2017) 829-842.

[89] A. Sewing, D. Cawkill, High-throughput lead finding and optimisation for GPCR targets, Ernst Schering Found Symp Proc, (2006) 249-267.

[90] K.B. Hansen, H. Brauner-Osborne, FLIPR assays of intracellular calcium in GPCR drug discovery, Methods in molecular biology, 552 (2009) 269-278.

[91] Q. Ma, L. Ye, H. Liu, Y. Shi, N. Zhou, An overview of Ca(2+) mobilization assays in GPCR drug discovery, Expert opinion on drug discovery, 12 (2017) 511-523.

[92] W.K. Kroeze, M.F. Sassano, X.P. Huang, K. Lansu, J.D. McCorvy, P.M. Giguere, N. Sciaky, B.L. Roth, PRESTO-Tango as an open-source resource for interrogation of the druggable human GPCRome, Nat Struct Mol Biol, 22 (2015) 362-U328.

[93] Y.M. Chen, Z.Y. Xu, D. Wu, J. Li, C. Song, W.Q. Lu, J. Huang, Luciferase Reporter Gene Assay on Human 5-HT Receptor: Which Response Element Should Be Chosen?, Scientific reports, 5 (2015).

[94] S.A. Martins, J.R. Trabuco, G.A. Monteiro, V. Chu, J.P. Conde, D.M. Prazeres, Towards the miniaturization of GPCR-based live-cell screening assays, Trends in biotechnology, 30 (2012) 566574. 
[95] M. Roelse, N.C. de Ruijter, E.X. Vrouwe, M.A. Jongsma, A generic microfluidic biosensor of G proteincoupled receptor activation-monitoring cytoplasmic $[\mathrm{Ca}(2+)]$ changes in human HEK293 cells, Biosensors \& bioelectronics, 47 (2013) 436-444.

[96] M.G. Henquet, M. Roelse, R.C. de Vos, A. Schipper, G. Polder, N.C. de Ruijter, R.D. Hall, M.A. Jongsma, Metabolomics meets functional assays: coupling LC-MS and microfluidic cell-based receptor-ligand analyses, Metabolomics : Official journal of the Metabolomic Society, 12 (2016) 115.

[97] T. Hart, A. Zhao, A. Garg, S. Bolusani, E.M. Marcotte, Human cell chips: adapting DNA microarray spotting technology to cell-based imaging assays, PloS one, 4 (2009) e7088.

[98] M.L. Yarmush, K.R. King, Living-cell microarrays, Annual review of biomedical engineering, 11 (2009) 235-257.

[99] M.H. Wu, S.B. Huang, G.B. Lee, Microfluidic cell culture systems for drug research, Lab on a chip, 10 (2010) 939-956.

[100] M. Roelse, M.G.L. Henquet, H.A. Verhoeven, N.C.A. de Ruijter, R. Wehrens, M.S. van Lenthe, R.F. Witkamp, R.D. Hall, M.A. Jongsma, Calcium Imaging of GPCR Activation Using Arrays of Reverse Transfected HEK293 Cells in a Microfluidic System, Sensors, 18 (2018).

[101] B.L. Roth, E. Lopez, S. Beischel, R.B. Westkaemper, J.M. Evans, Screening the receptorome to discover the molecular targets for plant-derived psychoactive compounds: a novel approach for CNS drug discovery, Pharmacol Ther, 102 (2004) 99-110.

[102] W.K. Kroeze, B.L. Roth, Screening the receptorome, J Psychopharmacol, 20 (2006) 41-46.

[103] X.R. Bao, I.D. Fraser, E.A. Wall, S.R. Quake, M.I. Simon, Variability in G-protein-coupled signaling studied with microfluidic devices, Biophysical journal, 99 (2010) 2414-2422.

[104] X. Zhang, H. Yin, J.M. Cooper, S.J. Haswell, A microfluidic-based system for analysis of single cells based on Ca2+ flux, Electrophoresis, 27 (2006) 5093-5100.

[105] R. Wehrens, M. Roelse, M. Henquet, M. van Lenthe, P.W. Goedhart, M.A. Jongsma, Statistical models discriminating between complex samples measured with microfluidic receptor-cell arrays, PloS one, 14 (2019) e0214878.

[106] M. Roelse, R. Wehrens, M.G.L. Henquet, R.F. Witkamp, R.D. Hall, M.A. Jongsma, The effect of calcium buffering and calcium sensor type on the sensitivity of an array-based bitter receptor screening assay., Chemical Senses (accepted), (2019).

[107] J.A. Stockert, L.A. Devi, Advancements in therapeutically targeting orphan GPCRs, Front Pharmacol, 6 (2015) 100.

[108] A. lida, S. Saito, A. Sekine, Y. Kataoka, W. Tabei, Y. Nakamura, Catalog of 300 SNPs in 23 genes encoding G-protein coupled receptors, J Hum Genet, 49 (2004) 194-208.

[109] A. lida, Y. Nakamura, Identification of 156 novel SNPs in 29 genes encoding G-protein coupled receptors, J Hum Genet, 50 (2005) 182-191.

[110] J.E. Hayes, E.L. Feeney, A.L. Allen, Do polymorphisms in chemosensory genes matter for human ingestive behavior?, Food quality and preference, 30 (2013) 202-216.

[111] R.a.M. Lappano, M., G protein-coupled receptors: novel targets for drug discovery in cancer, Nat. Rev. Drug Discov., 10 (2011) 47-60.

[112] D.M.e.a. Rosenbaum, The structure and function of G-protein- coupled receptors, Nature, 459 (2009) 356-363.

[113] I.L. Beets, M.; Janssen, T.; Verleyen, P., Deorphanizing G Protein-Coupled Receptors by a Calcium Mobilization Assay, in: A. Merighi (Ed.) Neuropeptides: Methods and Protocols, Methods in Molecular Biology, Springer Science2011.

[114] D.A. Yarmolinsky, C.S. Zuker, N.J.P. Ryba, Common Sense about Taste: From Mammals to Insects, Cell, 139 (2009) 234-244.

[115] H. Saito, Q.Y. Chi, H.Y. Zhuang, H. Matsunami, J.D. Mainland, Odor Coding by a Mammalian Receptor Repertoire, science signaling, 2 (2009).

[116] S.A.M.T. Martins, J.R.C.; Monteiro, G.A., Chu, V.; Conde, J.P.; Prazeres, D.M.F., Towards the miniturization of GPCR-based live-cell screening assays, Trends in Biotechnology, 989 (2012) 1-9.

[117] M.L.a.K. Yarmush, K.R., Living-cell microarrays, Annu. Rev. Biomed. Eng, 11 (2009) 235-257.

[118] M.H.H. Wu, S.B.; Lee, G.B., Microfluidic cell culture systems for drug research, Lab Chip, 10 (2010) 939-956. 
[119] K.J. Wieder, K.R. King, D.M. Thompson, C. Zia, M.L. Yarmush, A. Jayaraman, Optimization of Reporter Cells for Expression Profilingin a Microfluidic Device, Biomedical microdevices, 7:3 (2005) 213-222.

[120] J.A. Allen, B.L. Roth, Strategies to Discover Unexpected Targets for Drugs Active at G ProteinCoupled Receptors, Annu Rev Pharmacol, 51 (2011) 117-144.

[121] M.J.B. Berridge, M.D. and Roderick, L.H, Calcium signalling: dynamics, homeostasis and remodelling, Nature reviews, 4 (2003) 517-529.

[122] R.M.E. Paredes, J.C.; Watts, L.T.; Zheng, W; Leichleiter, J.D, Chemical calcium indicators, Elsevier methods, 46 (2008) 143-151.

[123] R. Yu, P.M. Hinkle, Rapid turnover of calcium in the endoplasmic reticulum during signaling. Studies with cameleon calcium indicators, The Journal of biological chemistry, 275 (2000) 23648-23653.

[124] E. Kostenis, M. Waelbroeck, G. Milligan, Techniques: Promiscuous G alpha proteins in basic research and drug discovery, Trends in Pharmacological Sciences, 26 (2005) 595-602.

[125] A. Miyawaki, J. Llopis, R. Heim, J.M. McCaffery, J.A. Adams, M. Ikura, R.Y. Tsien, Fluorescent indicators for Ca2+ based on green fluorescent proteins and calmodulin, Nature, 388 (1997) 882887.

[126] J.E. McCombs, A.E. Palmer, Measuring calcium dynamics in living cells with genetically encodable calcium indicators, Methods, 46 (2008) 152-159.

[127] A.E. Palmer, R.Y. Tsien, Measuring calcium signaling using genetically targetable fluorescent indicators, Nature protocols, 1 (2006) 1057-1065.

[128] A.E.Q. Palmer, Y.; Park, J.G.; McCombs, J.E., Design and application of genetically encoded biosensors, Trends in Biotechnology, 29 (2011) 144-152.

[129] Y.X. Zhao, S. Araki, W.H. Jiahui, T. Teramoto, Y.F. Chang, M. Nakano, A.S. Abdelfattah, M. Fujiwara, T. Ishihara, T. Nagai, R.E. Campbell, An Expanded Palette of Genetically Encoded Ca2+ Indicators, Science, 333 (2011) 1888-1891.

[130] J.W. Borst, S.P. Laptenok, A.H. Westphal, R. Kuhnemuth, H. Hornen, N.V. Visser, S. Kalinin, J. Aker, A. van Hoek, C.A. Seidel, A.J. Visser, Structural changes of yellow Cameleon domains observed by quantitative FRET analysis and polarized fluorescence correlation spectroscopy, Biophysical journal, 95 (2008) 5399-5411.

[131] T. Nagai, S. Yamada, T. Tominaga, M. Ichikawa, A. Miyawaki, Expanded dynamic range of fluorescent indicators for $\mathrm{Ca}(2+)$ by circularly permuted yellow fluorescent proteins, Proceedings of the National Academy of Sciences of the United States of America, 101 (2004) 10554-10559.

[132] P. de Felipe, G.A. Luke, L.E. Hughes, D. Gani, C. Halpin, M.D. Ryan, E unum pluribus: multiple proteins from a self-processing polyprotein, Trends in biotechnology, 24 (2006) 68-75.

[133] T. Hu, Q. Fu, P. Chen, K. Zhang, D. Guo, Generation of a stable mammalian cell line for simultaneous expression of multiple genes by using 2A peptide-based lentiviral vector, Biotechnology letters, 31 (2009) 353-359.

[134] J.v.W. Goedhart, L.; Adjobo-Hermans, M.J.W.; Elzenaar, I.; Hink, M.A.; Gadella, T.W.J, Quantitative co-expression of proteins ar the single cell level - application to a multimeric FRET sensor, PLos ONE, 6 (2011) 1-7.

[135] X.L. Zhang, H.B. Yin, J.M. Cooper, S.J. Haswell, A microfluidic-based system for analysis of single cells based on Ca2+ flux, Electrophoresis, 27 (2006) 5093-5100.

[136] X. Feng, J. Castracane, N. Tokranova, A. Gracias, G. Lnenicka, B.G. Szaro, , A living cell-based biosensor utilizing G-protein coupled receptors:

Principles and detection methods, Biosensors and Bioelectronics 22 (2007) 3230-3237.

[137] C.A. Maggi, The mammalian tachykinin receptors, General pharmacology, 26 (1995) 911-944.

[138] A.L. Szymczak, C.J. Workman, Y. Wang, K.M. Vignali, S. Dilioglou, E.F. Vanin, D.A. Vignali, Correction of multi-gene deficiency in vivo using a single 'self-cleaving' $2 \mathrm{~A}$ peptide-based retroviral vector, Nature biotechnology, 22 (2004) 589-594.

[139] V.J. Bennett, M.A. Simmons, Analysis of fluorescently labeled substance P analogs: binding, imaging and receptor activation, BMC chemical biology, 1 (2001) 1.

[140] E.S. Park, A.C. Brown, M.A. DiFeo, T.H. Barker, H. Lu, Continuously perfused, non-cross-contaminating microfluidic chamber array for studying cellular responses to orthogonal combinations of matrix and soluble signals, Lab on a Chip, 10 (2010) 571-580. 
[141] D.F. Gomez-Casati, M.I. Zanor, M.V. Busi, Metabolomics in plants and humans: applications in the prevention and diagnosis of diseases, Biomed Res Int, 2013 (2013) 792527.

[142] J. Tang, Microbial metabolomics, Current genomics, 12 (2011) 391-403.

[143] D.J. Creek, W.B. Dunn, O. Fiehn, J.L. Griffin, R.D. Hall, Z.T. Lei, R. Mistrik, S. Neumann, E.L. Schymanski, L.W. Sumner, R. Trengove, J.L. Wolfender, Metabolite identification: are you sure? And how do your peers gauge your confidence?, Metabolomics : Official journal of the Metabolomic Society, 10 (2014) 350-353.

[144] R.D. Hall, D.S. Wishart, U. Roessner, Metabolomics and the move towards biology, Metabolomics : Official journal of the Metabolomic Society, 7 (2011) 454-456.

[145] J.B. Regard, I.T. Sato, S.R. Coughlin, Anatomical profiling of G protein-coupled receptor expression, Cell, 135 (2008) 561-571.

[146] D.K. Vassilatis, J.G. Hohmann, H. Zeng, F. Li, J.E. Ranchalis, M.T. Mortrud, A. Brown, S.S. Rodriguez, J.R. Weller, A.C. Wright, J.E. Bergmann, G.A. Gaitanaris, The G protein-coupled receptor repertoires of human and mouse, Proceedings of the National Academy of Sciences of the United States of America, 100 (2003) 4903-4908.

[147] P. Tammela, T. Wennberg, H. Vuorela, P. Vuorela, HPLC micro-fractionation coupled to a cellbased assay for automated on-line primary screening of calcium antagonistic components in plant extracts, Anal Bioanal Chem, 380 (2004) 614-618.

[148] M.S. Butler, F. Fontaine, M.A. Cooper, Natural Product Libraries: Assembly, Maintenance, and Screening, Planta Med, 80 (2014) 1161-1170.

[149] T.A. Johnson, J. Sohn, W.D. Inman, S.A. Estee, S.T. Loveridge, H.C. Vervoort, K. Tenney, J.K. Liu, K.K.H. Ang, J. Ratnam, W.M. Bray, N.C. Gassner, Y.Y. Shen, R.S. Lokey, J.H. McKerrow, K. Boundy-Mills, A. Nukanto, A. Kanti, H. Julistiono, L.B.S. Kardono, L.F. Bjeldanes, P. Crews, Natural Product Libraries to Accelerate the High-Throughput Discovery of Therapeutic Leads, Journal of natural products, 74 (2011) 2545-2555.

[150] Y. Tu, C. Jeffries, H. Ruan, C. Nelson, D. Smithson, A.A. Shelat, K.M. Brown, X.C. Li, J.P. Hester, T. Smillie, I.A. Khan, L. Walker, K. Guy, B. Yan, Automated high-throughput system to fractionate plant natural products for drug discovery, J Nat Prod, 73 (2010) 751-754.

[151] M.K. Chung, A.D. Guler, M.J. Caterina, TRPV1 shows dynamic ionic selectivity during agonist stimulation, Nature neuroscience, 11 (2008) 555-564.

[152] Y. Wahyuni, A.R. Ballester, E. Sudarmonowati, R.J. Bino, A.G. Bovy, Metabolite biodiversity in pepper (Capsicum) fruits of thirty-two diverse accessions: variation in health-related compounds and implications for breeding, Phytochemistry, 72 (2011) 1358-1370.

[153] J.J. van der Hooft, R.C. de Vos, V. Mihaleva, R.J. Bino, L. Ridder, N. de Roo, D.M. Jacobs, J.P. van Duynhoven, J. Vervoort, Structural elucidation and quantification of phenolic conjugates present in human urine after tea intake, Anal Chem, 84 (2012) 7263-7271.

[154] Y. Wahyuni, A.R. Ballester, Y. Tikunov, R.C. de Vos, K.T. Pelgrom, A. Maharijaya, E. Sudarmonowati, R.J. Bino, A.G. Bovy, Metabolomics and molecular marker analysis to explore pepper (Capsicum sp.) biodiversity, Metabolomics : Official journal of the Metabolomic Society, 9 (2013) 130-144.

[155] K. Novakova-Tousova, L. Vyklicky, K. Susankova, J. Benedikt, A. Samad, J. Teisinger, V. Vlachova, Functional changes in the vanilloid receptor subtype 1 channel during and after acute desensitization, Neuroscience, 149 (2007) 144-154.

[156] F. Touska, L. Marsakova, J. Teisinger, V. Vlachova, A "cute" desensitization of TRPV1, Curr Pharm Biotechnol, 12 (2011) 122-129.

[157] J. Jung, S.W. Hwang, J. Kwak, S.Y. Lee, C.J. Kang, W.B. Kim, D. Kim, U. Oh, Capsaicin binds to the intracellular domain of the capsaicin-activated ion channel, The Journal of neuroscience : the official journal of the Society for Neuroscience, 19 (1999) 529-538.

[158] K. Hui, B. Liu, F. Qin, Capsaicin activation of the pain receptor, VR1: multiple open states from both partial and full binding, Biophysical journal, 84 (2003) 2957-2968.

[159] L. Vyklicky, A. Lyfenko, D.P. Kuffler, V. Vlachova, Vanilloid receptor TRPV1 is not activated by vanilloids applied intracellularly, Neuroreport, 14 (2003) 1061-1065.

[160] O. Bountagkidou, E.J.C. van der Klift, M.Z. Tsimidou, S.A. Ordoudi, T.A. van Beek, An on-line high performance liquid chromatography-crocin bleaching assay for detection of antioxidants, J Chromatogr A, 1237 (2012) 80-85. 
[161] H.A. Niederlander, T.A. van Beek, A. Bartasiute, Koleva, II, Antioxidant activity assays on-line with liquid chromatography, J Chromatogr A, 1210 (2008) 121-134.

[162] J. Beekwilder, R.D. Hall, C.H. de Vos, Identification and dietary relevance of antioxidants from raspberry, Biofactors, 23 (2005) 197-205.

[163] J.W. Schoonen, P. Vulto, N. de Roo, J. van Duynhoven, H. van der Linden, T. Hankemeier, Solvent exchange module for LC-NMR hyphenation using machine vision-controlled droplet evaporation, Analytical chemistry, 85 (2013) 5734-5739.

[164] S.N. Bailey, D.M. Sabatini, B.R. Stockwell, Microarrays of small molecules embedded in biodegradable polymers for use in mammalian cell-based screens, Proceedings of the National Academy of Sciences of the United States of America, 101 (2004) 16144-16149.

[165] S.N. Bailey, S.M. Ali, A.E. Carpenter, C.O. Higgins, D.M. Sabatini, Microarrays of lentiviruses for gene function screens in immortalized and primary cells, Nature methods, 3 (2006) 117-122.

[166] S. Rajan, H. Djambazian, H.C. Dang, R. Sladek, T.J. Hudson, The living microarray: a high-throughput platform for measuring transcription dynamics in single cells, BMC genomics, 12 (2011) 115.

[167] J. Tian, S. Alimperti, P. Lei, S.T. Andreadis, Lentiviral microarrays for real-time monitoring of gene expression dynamics, Lab on a chip, 10 (2010) 1967-1975.

[168] R. Onuki-Nagasaki, A. Nagasaki, K. Hakamada, T.Q. Uyeda, S. Fujita, M. Miyake, J. Miyake, On-chip screening method for cell migration genes based on a transfection microarray, Lab on a chip, 8 (2008) 1502-1506.

[169] S. Fujita, R. Onuki-Nagasaki, K. Ikuta, Y. Hara, A simple method for producing multiple copies of controlled release small molecule microarrays for cell-based screening, Biofabrication, 9 (2016) 011001.

[170] T. Niidome, K. Yakumaru, A. Shiotani, S. Yamashita, Y. Katayama, Gene Transfer into Cells from Solid Surfaces and Its Application to In Vivo Systems, Chem Lett, 38 (2009) 36-37.

[171] M. Miyake, T. Yoshikawa, S. Fujita, J. Miyake, Transfection microarray and the applications, Molecular bioSystems, 5 (2009) 444-449.

[172] C. Kneuer, C. Ehrhardt, H. Bakowsky, M.N. Kumar, V. Oberle, C.M. Lehr, D. Hoekstra, U. Bakowsky, The influence of physicochemical parameters on the efficacy of non-viral DNA transfection complexes: a comparative study, Journal of nanoscience and nanotechnology, 6 (2006) 2776-2782.

[173] Z. Bengali, J.C. Rea, R.F. Gibly, L.D. Shea, Efficacy of immobilized polyplexes and lipoplexes for substrate-mediated gene delivery, Biotechnology and bioengineering, 102 (2009) 1679-1691.

[174] P.L. Felgner, T.R. Gadek, M. Holm, R. Roman, H.W. Chan, M. Wenz, J.P. Northrop, G.M. Ringold, M. Danielsen, Lipofection: a highly efficient, lipid-mediated DNA-transfection procedure, Proceedings of the National Academy of Sciences of the United States of America, 84 (1987) 7413-7417.

[175] S. Brunner, T. Sauer, S. Carotta, M. Cotten, M. Saltik, E. Wagner, Cell cycle dependence of gene transfer by lipoplex, polyplex and recombinant adenovirus, Gene therapy, 7 (2000) 401-407.

[176] D. Lechardeur, K.J. Sohn, M. Haardt, P.B. Joshi, M. Monck, R.W. Graham, B. Beatty, J. Squire, H. O'Brodovich, G.L. Lukacs, Metabolic instability of plasmid DNA in the cytosol: a potential barrier to gene transfer, Gene therapy, 6 (1999) 482-497.

[177] R.N. Cohen, M.A. van der Aa, N. Macaraeg, A.P. Lee, F.C. Szoka, Jr., Quantification of plasmid DNA copies in the nucleus after lipoplex and polyplex transfection, Journal of controlled release : official journal of the Controlled Release Society, 135 (2009) 166-174.

[178] L. Fliedl, F. Kast, J. Grillari, M. Wieser, R. Grillari-Voglauer, Optimization of a quantitative PCR based method for plasmid copy number determination in human cell lines, New biotechnology, 32 (2015) 716-719.

[179] M. Soni, F. Lai, Cell-based co-transfection microarrays for use with HEK293T cells on a poly D-lysinecoated polystyrene microplate, Methods in molecular biology, 706 (2011) 13-25.

[180] J. De La Vega, B.T. Braak, A.R. Azzoni, G.A. Monteiro, D.M. Prazeres, Impact of plasmid quality on lipoplex-mediated transfection, Journal of pharmaceutical sciences, 102 (2013) 3932-3941.

[181] J. Enomoto, R. Takagi, R. Onuki-Nagasaki, S. Fujita, J. Fukuda, Reverse transfection in microchamber arrays for cell migration assays, Sensor Actuat B-Chem, 190 (2014) 896-899.

[182] T. Jain, R. McBride, S. Head, E. Saez, Highly parallel introduction of nucleic acids into mammalian cells grown in microwell arrays, Lab on a chip, 9 (2009) 3557-3566. 
[183] B.R. Schudel, B. Harmon, V.V. Abhyankar, B.W. Pruitt, O.A. Negrete, A.K. Singh, Microfluidic platforms for RNA interference screening of virus-host interactions, Lab on a chip, 13 (2013) 811-817.

[184] A.E. Palmer, Y. Qin, J.G. Park, J.E. McCombs, Design and application of genetically encoded biosensors, Trends in biotechnology, 29 (2011) 144-152.

[185] L. Kamentsky, T.R. Jones, A. Fraser, M.A. Bray, D.J. Logan, K.L. Madden, V. Ljosa, C. Rueden, K.W. Eliceiri, A.E. Carpenter, Improved structure, function and compatibility for CellProfiler: modular high-throughput image analysis software, Bioinformatics, 27 (2011) 1179-1180.

[186] S.M. Haghparast, T. Kihara, J. Miyake, Distinct mechanical behavior of HEK293 cells in adherent and suspended states, PeerJ, 3 (2015) e1131.

[187] L. Erb, G.A. Weisman, Coupling of P2Y receptors to G proteins and other signaling pathways, Wiley interdisciplinary reviews. Membrane transport and signaling, 1 (2012) 789-803.

[188] J. Zabner, A.J. Fasbender, T. Moninger, K.A. Poellinger, M.J. Welsh, Cellular and molecular barriers to gene transfer by a cationic lipid, The Journal of biological chemistry, 270 (1995) 18997-19007.

[189] W. Krek, J.A. DeCaprio, Cell synchronization, Methods in enzymology, 254 (1995) 114-124.

[190] M.G. Kauffman, S.J. Noga, T.J. Kelly, A.D. Donnenberg, Isolation of cell cycle fractions by counterflow centrifugal elutriation, Analytical biochemistry, 191 (1990) 41-46.

[191] G.F. Ross, M.D. Bruno, M. Uyeda, K. Suzuki, K. Nagao, J.A. Whitsett, T.R. Korfhagen, Enhanced reporter gene expression in cells transfected in the presence of DMI-2, an acid nuclease inhibitor, Gene therapy, 5 (1998) 1244-1250.

[192] R. Moriguchi, K. Kogure, A. Iwasa, H. Akita, H. Harashima, Non-linear pharmacodynamics in a non-viral gene delivery system: positive non-linear relationship between dose and transfection efficiency, Journal of controlled release : official journal of the Controlled Release Society, 110 (2006) 605-609.

[193] A. Varrault, L. Journot, Y. Audigier, J. Bockaert, Transfection of human 5-hydroxytryptamine1A receptors in NIH-3T3 fibroblasts: effects of increasing receptor density on the coupling of 5-hydroxytryptamine1A receptors to adenylyl cyclase, Molecular pharmacology, 41 (1992) 9991007.

[194] M. Behrens, W. Meyerhof, Bitter taste receptor research comes of age: from characterization to modulation of TAS2Rs, Semin Cell Dev Biol, 24 (2013) 215-221.

[195] P. Devillier, E. Naline, S. Grassin-Delyle, The pharmacology of bitter taste receptors and their role in human airways, Pharmacol Ther, 155 (2015) 11-21.

[196] B. Bufe, E. Scholey-Pohl, D. Krautwurst, T. Hofmann, W. Meyerhof, Identification of human bitter taste receptors, Acs Sym Ser, 867 (2004) 45-59.

[197] M. Behrens, J. Bartelt, C. Reichling, M. Winnig, C. Kuhn, W. Meyerhof, Members of RTP and REEP gene families influence functional bitter taste receptor expression, The Journal of biological chemistry, 281 (2006) 20650-20659.

[198] C. Reichling, W. Meyerhof, M. Behrens, Functions of human bitter taste receptors depend on N-glycosylation, J Neurochem, 106 (2008) 1138-1148.

[199] C. Kuhn, B. Bufe, C. Batram, W. Meyerhof, Oligomerization of TAS2R bitter taste receptors, Chemical senses, 35 (2010) 395-406.

[200] A. Miyawaki, T. Nagai, H. Mizuno, Imaging intracellular free Ca2+ concentration using yellow cameleons, Cold Spring Harbor protocols, 2013 (2013).

[201] Y. Zhao, S. Araki, J. Wu, T. Teramoto, Y.F. Chang, M. Nakano, A.S. Abdelfattah, M. Fujiwara, T. Ishihara, T. Nagai, R.E. Campbell, An expanded palette of genetically encoded $\mathrm{Ca}(2)(+)$ indicators, Science, 333 (2011) 1888-1891.

[202] M. Mank, O. Griesbeck, Genetically encoded calcium indicators, Chem Rev, 108 (2008) 1550-1564.

[203] J. Goedhart, D. von Stetten, M. Noirclerc-Savoye, M. Lelimousin, L. Joosen, M.A. Hink, L. van Weeren, T.W. Gadella, Jr., A. Royant, Structure-guided evolution of cyan fluorescent proteins towards a quantum yield of 93\%, Nature communications, 3 (2012) 751.

[204] K. Horikawa, Y. Yamada, T. Matsuda, K. Kobayashi, M. Hashimoto, T. Matsu-ura, A. Miyawaki, T. Michikawa, K. Mikoshiba, T. Nagai, Spontaneous network activity visualized by ultrasensitive $\mathrm{Ca}(2+)$ indicators, yellow Cameleon-Nano, Nature methods, 7 (2010) 729-732. 
[205] T. Thestrup, J. Litzlbauer, I. Bartholomaus, M. Mues, L. Russo, H. Dana, Y. Kovalchuk, Y. Liang, G. Kalamakis, Y. Laukat, S. Becker, G. Witte, A. Geiger, T. Allen, L.C. Rome, T.W. Chen, D.S. Kim, O. Garaschuk, C. Griesinger, O. Griesbeck, Optimized ratiometric calcium sensors for functional in vivo imaging of neurons and T lymphocytes, Nature methods, 11 (2014) 175-182.

[206] J. Tong, T.V. McCarthy, D.H. MacLennan, Measurement of resting cytosolic Ca2+ concentrations and Ca2+ store size in HEK-293 cells transfected with malignant hyperthermia or central core disease mutant Ca2+ release channels, The Journal of biological chemistry, 274 (1999) 693-702.

[207] T. Rose, P.M. Goltstein, R. Portugues, O. Griesbeck, Putting a finishing touch on GECls, Frontiers in molecular neuroscience, 7 (2014) 88.

[208] S.R. Gadagkar, G.B. Call, Computational tools for fitting the Hill equation to dose-response curves, J Pharmacol Toxicol Methods, 71 (2015) 68-76.

[209] K. Lossow, S. Hubner, N. Roudnitzky, J.P. Slack, F. Pollastro, M. Behrens, W. Meyerhof, Comprehensive Analysis of Mouse Bitter Taste Receptors Reveals Different Molecular Receptive Ranges for Orthologous Receptors in Mice and Humans, The Journal of biological chemistry, 291 (2016) 1535815377.

[210] M. Narukawa, C. Noga, Y. Ueno, T. Sato, T. Misaka, T. Watanabe, Evaluation of the bitterness of green tea catechins by a cell-based assay with the human bitter taste receptor hTAS2R39, Biochem Biophys Res Commun, 405 (2011) 620-625.

[211] N. Wettschureck, S. Offermanns, Mammalian G proteins and their cell type specific functions, Physiol Rev, 85 (2005) 1159-1204.

[212] S. Born, A. Levit, M.Y. Niv, W. Meyerhof, M. Behrens, The Human Bitter Taste Receptor TAS2R10 Is Tailored to Accommodate Numerous Diverse Ligands, Journal of Neuroscience, 33 (2013) 201-213.

[213] M. Behrens, A. Brockhoff, C. Kuhn, B. Bufe, M. Winnig, W. Meyerhof, The human taste receptor hTAS2R14 responds to a variety of different bitter compounds, Biochem Biophys Res Commun, 319 (2004) 479-485.

[214] T.A. Greene, S. Alarcon, A. Thomas, E. Berdougo, B.J. Doranz, P.A. Breslin, J.B. Rucker, Probenecid inhibits the human bitter taste receptor TAS2R16 and suppresses bitter perception of salicin, PloS one, 6 (2011) e20123.

[215] B. Bufe, T. Hofmann, D. Krautwurst, J.D. Raguse, W. Meyerhof, The human TAS2R16 receptor mediates bitter taste in response to beta-glucopyranosides, Nat Genet, 32 (2002) 397-401.

[216] W. Meyerhof, S. Born, A. Brockhoff, M. Behrens, Molecular biology of mammalian bitter taste receptors. A review, Flavour Frag J, 26 (2011) 260-268.

[217] C. Kuhn, B. Bufe, M. Winnig, T. Hofmann, O. Frank, M. Behrens, T. Lewtschenko, J.P. Slack, C.D. Ward, W. Meyerhof, Bitter taste receptors for saccharin and acesulfame K, Journal of Neuroscience, 24 (2004) 10260-10265.

[218] A. Brockhoff, M. Behrens, A. Massarotti, G. Appendino, W. Meyerhof, Broad tuning of the human bitter taste receptor hTAS2R46 to various sesquiterpene lactones, clerodane and labdane diterpenoids, strychnine, and denatonium, Journal of agricultural and food chemistry, 55 (2007) 6236-6243.

[219] B. Schwaller, Cytosolic Ca2+ buffers, Cold Spring Harb Perspect Biol, 2 (2010) a004051.

[220] D.E. Clapham, Calcium signaling, Cell, 131 (2007) 1047-1058.

[221] S.M. McMahon, M.B. Jackson, An Inconvenient Truth: Calcium Sensors Are Calcium Buffers, Trends Neurosci, 41 (2018) 880-884.

[222] J. Yang, D. Cumberbatch, S. Centanni, S.Q. Shi, D. Winder, D. Webb, C.H. Johnson, Coupling optogenetic stimulation with NanoLuc-based luminescence (BRET) $\mathrm{Ca}(++)$ sensing, Nature communications, 7 (2016) 13268.

[223] S.B. Kim, Bioluminescence: Methods and Protocols, Springer Science+Business Media New York, New York, 2016.

[224] Q. Ni, S. Mehta, J. Zhang, Live-cell imaging of cell signaling using genetically encoded fluorescent reporters, FEBS J, 285 (2018) 203-219.

[225] L. Lindenburg, M. Merkx, Engineering genetically encoded FRET sensors, Sensors, 14 (2014) 1169111713.

[226] E. Nishihara, T.Y. Hiyama, M. Noda, Osmosensitivity of Transient Receptor Potential Vanilloid 1 Is Synergistically Enhanced by Distinct Activating Stimuli Such as Temperature and Protons, PloS one, 6 (2011). 
[227] M. Bauer, K. Kim, Y.L. Qiu, B. Calpe, A. Khadeinhosseini, R.L. Liao, I. Wheeldon, Spot Identification and Quality Control in Cell-Based Microarrays, Acs Comb Sci, 14 (2012) 471-477.

[228] C.S. Wu, Y.W. Du, L.Q. Huang, Y.B. Galeczki, A. Dagan-Wiener, M. Naim, M.Y. Niv, P. Wang, Biomimetic Sensors for the Senses: Towards Better Understanding of Taste and Odor Sensation, Sensors, 17 (2017).

[229] M. Lee, H. Yang, D. Kim, M. Yang, T.H. Park, S. Hong, Human-like smelling of a rose scent using an olfactory receptor nanodisc-based bioelectronic nose, Scientific reports, 8 (2018).

[230] H.S. Song, H.J. Jin, S.R. Ahn, D. Kim, S.H. Lee, U.K. Kim, C.T. Simons, S. Hong, T.H. Park, Bioelectronic Tongue Using Heterodimeric Human Taste Receptor for the Discrimination of Sweeteners with Human-like Performance, Acs Nano, 8 (2014) 9781-9789.

[231] M.S. Butler, F. Fontaine, M.A. Cooper, Natural product libraries: assembly, maintenance, and screening, Planta Med, 80 (2014) 1161-1170.

[232] J. Chambers, T. Hastie, Statistical Models in S, Chapman and Hall/CRC1991.

[233] J. Jackson, A User's Guide to Principal Components, Wiley-Blackwell1991.

[234] R. Wehrens, Chemometrics with R - Multivariate Data Analysis in the Natural Sciences and Life Sciences, Springer2011.

[235] J. Pinheiro, D. Bates, Mixed-Effects Models in S and S-PLUS. Statistics and Computing, Springer 2006.

[236] R-Core-team, R: A language and environment for statistical computing, R Foundation for Statistical Computing, Vienna, Austria, 2014.

[237] J. Pinheiro, D. Bates, S. DebRoy, D. Sarkar, R.C. Team, nlme: Linear and Nonlinear Mixed Effects Models., 2019.

[238] R. Lenth, emmeans: Estimated Marginal Means, aka Least-Squares Means, 2018.

[239] D. Sarkar, Multivariate Data Visualization with R. , Springer2008.

[240] R.T. Strachan, G. Ferrara, B.L. Roth, Screening the receptorome: an efficient approach for drug discovery and target validation, Drug discovery today, 11 (2006) 708-716.

[241] M. Sumit, S. Takayama, J.J. Linderman, New insights into mammalian signaling pathways using microfluidic pulsatile inputs and mathematical modeling, Integrative biology : quantitative biosciences from nano to macro, 9 (2017) 6-21.

[242] A. Jovic, B. Howell, M. Cote, S.M. Wade, K. Mehta, A. Miyawaki, R.R. Neubig, J.J. Linderman, S. Takayama, Phase-locked signals elucidate circuit architecture of an oscillatory pathway, PLoS computational biology, 6 (2010) e1001040.

[243] M.C. Hendriks-Balk, S.L. Peters, M.C. Michel, A.E. Alewijnse, Regulation of G protein-coupled receptor signalling: focus on the cardiovascular system and regulator of $G$ protein signalling proteins, European journal of pharmacology, 585 (2008) 278-291.

[244] R.M. Richardson, R.J. Marjoram, L.S. Barak, R. Snyderman, Role of the cytoplasmic tails of CXCR1 and CXCR2 in mediating leukocyte migration, activation, and regulation, J Immunol, 170 (2003) 2904-2911.

[245] M. Roelse, M. Henquet, M. Jongsma, Receptomics: Calcium imaging of sensory receptor cell arrays in a microfluidic system and novel applications for food screening, Chemical senses, 44 (2019) E33-E34.

[246] B. David, J.L. Wolfender, D.A. Dias, The pharmaceutical industry and natural products: historical status and new trends, Phytochem Rev, 14 (2015) 299-315.

[247] P. Norman, The Changing Role of Natural Products in Drug Discovery, Business Insights, Business Insights, 2011, pp. 1-125.

[248] J.D. Phillipson, Phytochemistry and pharmacognosy, Phytochemistry, 68 (2007) 2960-2972.

[249] F.E. Koehn, G.T. Carter, The evolving role of natural products in drug discovery, Nature Reviews Drug Discovery, 4 (2005) 206-220.

[250] C. Hellfritsch, A. Brockhoff, F. Stahler, W. Meyerhof, T. Hofmann, Human psychometric and taste receptor responses to steviol glycosides, Journal of agricultural and food chemistry, 60 (2012) 67826793.

[251] D.J. Chalmers, K.S. Rowan, The climacteric in ripening tomato fruit, Plant Physiol, 48 (1971) 235-240.

[252] A. Sorrequieta, G. Ferraro, S.B. Boggio, E.M. Valle, Free amino acid production during tomato fruit ripening: a focus on L-glutamate, Amino Acids, 38 (2010) 1523-1532. 
[253] K.A. Jacobson, A.A. Ivanov, S. de Castro, T.K. Harden, H. Ko, Development of selective agonists and antagonists of P2Y receptors, Purinergic Signal, 5 (2009) 75-89.

[254] A. Salahpour, S. Espinoza, B. Masri, V. Lam, L.S. Barak, R.R. Gainetdinov, BRET biosensors to study GPCR biology, pharmacology, and signal transduction, Frontiers in endocrinology, 3 (2012) 105.

[255] B.D. Shepard, N. Natarajan, R.J. Protzko, O.W. Acres, J.L. Pluznick, A cleavable N-terminal signal peptide promotes widespread olfactory receptor surface expression in HEK293T cells, PloS one, 8 (2013) e68758.

[256] F. Noe, T. Frey, J. Fiedler, C. Geithe, B. Nowak, D. Krautwurst, IL-6-HaloTag ${ }^{\circledR}$ enables live-cell plasma membrane staining, flow cytometry, functional expression, and de-orphaning of recombinant odorant receptors, J Biol Methods, 4(4):e81. (2017).

[257] Z. Peterlin, S. Firestein, M.E. Rogers, The state of the art of odorant receptor deorphanization: a report from the orphanage, The Journal of general physiology, 143 (2014) 527-542.

[258] Y.R. Li, H. Matsunami, Activation state of the M3 muscarinic acetylcholine receptor modulates mammalian odorant receptor signaling, Science signaling, 4 (2011) ra1.

[259] Y. Jiang, Y.R. Li, H. Tian, M. Ma, H. Matsunami, Muscarinic acetylcholine receptor M3 modulates odorant receptor activity via inhibition of beta-arrestin-2 recruitment, Nature communications, 6 (2015) 6448.

[260] Q. Wan, N. Okashah, A. Inoue, R. Nehme, B. Carpenter, C.G. Tate, N.A. Lambert, Mini G protein probes for active $\mathrm{G}$ protein-coupled receptors (GPCRs) in live cells, The Journal of biological chemistry, 293 (2018) 7466-7473.

[261] S.C. Kinnamon, T.E. Finger, A taste for ATP: neurotransmission in taste buds, Front Cell Neurosci, 7 (2013) 264.

[262] S.G. Huang, Development of a high throughput screening assay for mitochondrial membrane potential in living cells, Journal of biomolecular screening, 7 (2002) 383-389.

[263] A. Vongs, K.J. Solly, L. Kiss, D.J. Macneil, C.I. Rosenblum, A miniaturized homogenous assay of mitochondrial membrane potential, Assay and drug development technologies, 9 (2011) 373-381.

[264] S. Sakamuru, X. Li, M.S. Attene-Ramos, R. Huang, J. Lu, L. Shou, M. Shen, R.R. Tice, C.P. Austin, M. Xia, Application of a homogenous membrane potential assay to assess mitochondrial function, Physiological genomics, 44 (2012) 495-503.

[265] P. Coward, S.D. Chan, H.G. Wada, G.M. Humphries, B.R. Conklin, Chimeric G proteins allow a highthroughput signaling assay of Gi-coupled receptors, Analytical biochemistry, 270 (1999) 242-248.

[266] E. Kostenis, G proteins in drug screening: from analysis of receptor-G protein specificity to manipulation of GPCR-mediated signalling pathways, Current pharmaceutical design, 12 (2006) 1703-1715.

[267] Z. Chilmonczyk, A.J. Bojarski, I. Sylte, Ligand-directed trafficking of receptor stimulus, Pharmacological reports : PR, 66 (2014) 1011-1021.

[268] D. Bolognini, C.E. Moss, K. Nilsson, A.U. Petersson, I. Donnelly, E. Sergeev, G.M. Konig, E. Kostenis, M. Kurowska-Stolarska, A. Miller, N. Dekker, A.B. Tobin, G. Milligan, A Novel Allosteric Activator of Free Fatty Acid 2 Receptor Displays Unique Gi-functional Bias, The Journal of biological chemistry, 291 (2016) 18915-18931.

[269] J. Klarenbeek, J. Goedhart, A. van Batenburg, D. Groenewald, K. Jalink, Fourth-generation epacbased FRET sensors for CAMP feature exceptional brightness, photostability and dynamic range: characterization of dedicated sensors for FLIM, for ratiometry and with high affinity, PloS one, 10 (2015) e0122513.

[270] M. Maziarz, M. Garcia-Marcos, Rapid kinetic BRET measurements to monitor G protein activation by GPCR and non-GPCR proteins, Methods Cell Biol, 142 (2017) 145-157.

[271] J. Goedhart, L. van Weeren, M.J. Adjobo-Hermans, I. Elzenaar, M.A. Hink, T.W. Gadella, Jr., Quantitative co-expression of proteins at the single cell level--application to a multimeric FRET sensor, PloS one, 6 (2011) e27321.

[272] R. Nehme, B. Carpenter, A. Singhal, A. Strege, P.C. Edwards, C.F. White, H. Du, R. Grisshammer, C.G. Tate, Mini-G proteins: Novel tools for studying GPCRs in their active conformation, PloS one, 12 (2017) e0175642.

[273] H. Erfle, B. Neumann, U. Liebel, P. Rogers, M. Held, T. Walter, J. Ellenberg, R. Pepperkok, Reverse transfection on cell arrays for high content screening microscopy, Nature protocols, 2 (2007) 392399. 
[274] E. Ueda, P.A. Levkin, Emerging Applications of Superhydrophilic-Superhydrophobic Micropatterns, Adv Mater, 25 (2013) 1234-1247.

[275] D. Zhang, Q. Liu, Biosensors and bioelectronics on smartphone for portable biochemical detection, Biosensors \& bioelectronics, 75 (2016) 273-284.

[276] A. Roda, E. Michelini, M. Zangheri, M. Di Fusco, D. Calabria, P. Simoni, Smartphone-based biosensors: A critical review and perspectives, Trac-Trend Anal Chem, 79 (2016) 317-325.

[277] K.I. Liszt, J.P. Ley, B. Rohm, V. Stoeger, E. Koeck, A. Stuebler, C.M. Hochkogler, M.M. Somoza, S. Widder, J. Hans, V. Somoza, Caffeine-induced activation of oral and gastric bitter taste receptors regulates gastric acid secretion, Chemical senses, 40 (2015) 602-603.

[278] K.I. Liszt, J. Hans, J.P. Ley, E. Kock, V. Somoza, Characterization of Bitter Compounds via Modulation of Proton Secretion in Human Gastric Parietal Cells in Culture, Journal of agricultural and food chemistry, 66 (2018) 2295-2300.

[279] A. Chavez, J. Scheiman, S. Vora, B.W. Pruitt, M. Tuttle, P.R.I. E, S. Lin, S. Kiani, C.D. Guzman, D.J. Wiegand, D. Ter-Ovanesyan, J.L. Braff, N. Davidsohn, B.E. Housden, N. Perrimon, R. Weiss, J. Aach, J.J. Collins, G.M. Church, Highly efficient Cas9-mediated transcriptional programming, Nature methods, 12 (2015) 326-328.

[280] M. Spehr, G. Gisselmann, A. Poplawski, J.A. Riffell, C.H. Wetzel, R.K. Zimmer, H. Hatt, Identification of a testicular odorant receptor mediating human sperm chemotaxis, Science, 299 (2003) 20542058.

[281] J. Walsh, A. Cram, K. Woertz, J. Breitkreutz, G. Winzenburg, R. Turner, C. Tuleu, I. European Formulation, Playing hide and seek with poorly tasting paediatric medicines: do not forget the excipients, Advanced drug delivery reviews, 73 (2014) 14-33.

[282] H. Sohi, Y. Sultana, R.K. Khar, Taste masking technologies in oral pharmaceuticals: recent developments and approaches, Drug development and industrial pharmacy, 30 (2004) 429-448.

[283] J.P. Ley, Masking Bitter Taste by Molecules, Chemosens Percept, 1 (2008) 58-77.

[284] J.P. Ley, G. Krammer, G. Reinders, I.L. Gatfield, H.J. Bertram, Evaluation of bitter masking flavanones from Herba Santa (Eriodictyon californicum (H. and A.) Torr., Hydrophyllaceae), Journal of agricultural and food chemistry, 53 (2005) 6061-6066.

[285] L.M. Bartoshuk, H.J. Klee, Better Fruits and Vegetables through Sensory Analysis, Curr Biol, 23 (2013) R374-R378.

[286] M. Son, D. Kim, H.J. Ko, S. Hong, T.H. Park, A portable and multiplexed bioelectronic sensor using human olfactory and taste receptors, Biosensors \& bioelectronics, 87 (2017) 901-907.

[287] M. Son, T.H. Park, The bioelectronic nose and tongue using olfactory and taste receptors: Analytical tools for food quality and safety assessment, Biotechnol Adv, 36 (2018) 371-379.

[288] C. Trimmer, A. Keller, N.R. Murphy, L.L. Snyder, J.R. Willer, M.H. Nagai, N. Katsanis, L.B. Vosshall, H. Matsunami, J.D. Mainland, Genetic variation across the human olfactory receptor repertoire alters odor perception, Proceedings of the National Academy of Sciences of the United States of America, 116 (2019) 9475-9480.

[289] T. Hofmann, Identification of the key bitter compounds in our daily diet is a prerequisite for the understanding of the hTAS2R gene polymorphisms affecting food choice, Ann N Y Acad Sci, 1170 (2009) 116-125.

[290] M. Di Sanzo, L. Cipolloni, M. Borro, R. La Russa, A. Santurro, M. Scopetti, M. Simmaco, P. Frati, Clinical Applications of Personalized Medicine: A New Paradigm and Challenge, Curr Pharm Biotechnol, 18 (2017) 194-203.

[291] A. Middleton, Society and personal genome data, Hum Mol Genet, 27 (2018) R8-R13. 

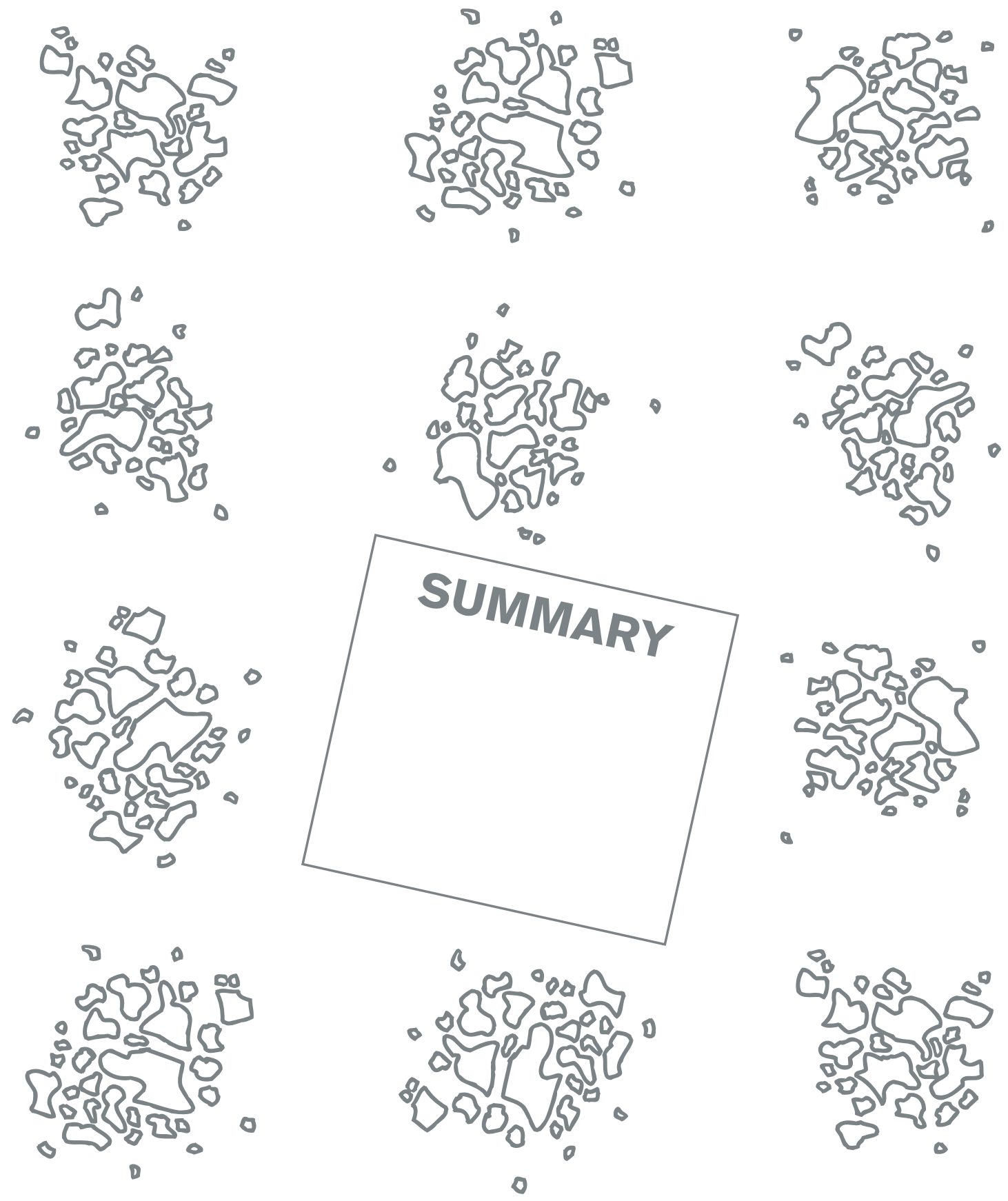

a
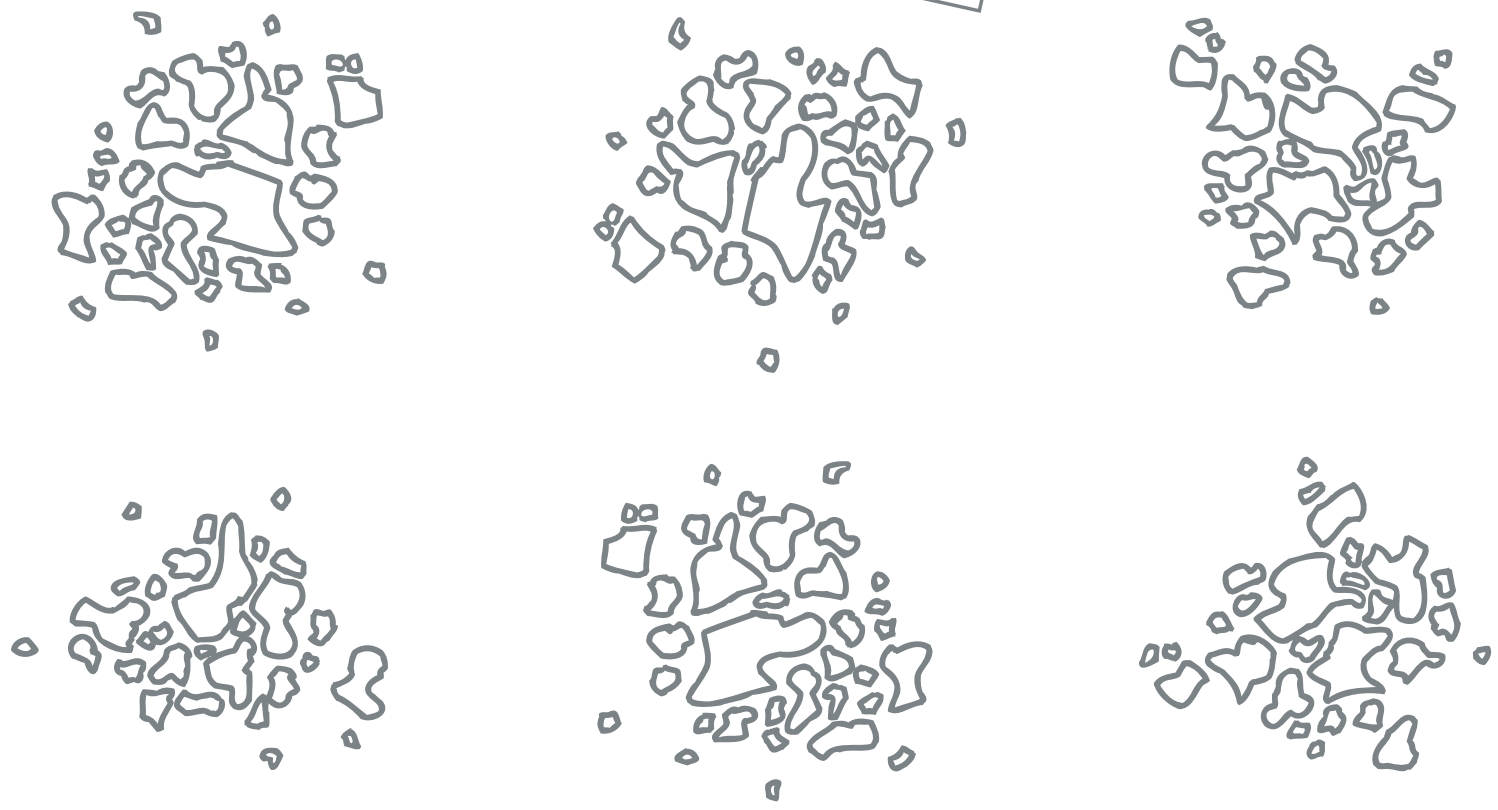
This thesis describes the development of a G Protein-Coupled Receptor (GPCR) screening technology that combines a receptor cell array ( $\sim 300$ spots) with microfluidics. This technology was developed for the purpose of sensing the taste of, or active components in complex samples. GPCR activation was monitored using a genetically encoded calcium indicator (GECI) which was based on a change in Förster Resonance Energy Transfer (FRET) between two fluorescent proteins linked by a calcium binding domain which, upon binding of calcium, induces a conformational change between the fluorophores. The receptor cell arrays were created by reverse transfection of printed plasmid DNA. The arrays were assembled in a flowcell, connected to a microfluidic system, and mounted on a stereo fluorescence microscope. This setup allowed for controlled and importantly, repeated sample exposure while monitoring the changes in intracellular calcium in real-time.

GPCRs play an important role in many physiological or disease-related processes. These membrane proteins have evolved to sense a wide range of molecules that can be of either exogenous or endogenous origin. Their sensing mechanisms are complex and potentially involve many cellular signalling events depending the cell type. The introductory chapter of this thesis presents a brief overview of the GPCR types and their signalling pathways with a focus on taste signalling. This chapter also places the microfluidic receptomics technology within the framework of existing receptor screening technologies.

The second chapter explores the general principles, setup and characterization of the microfluidic biosensor to measure GPCR activation via imaging of $\left[\mathrm{Ca}^{2+}\right]$ changes in recombinant human HEK293 cells. These cells expressed a combination of the Neurokinin 1-receptor and Cameleon YC3.6 protein as calcium indicator. Here, a stable cell line was employed for robust expression with little variation

Next to GPCRs, the system was also used for the detection of transient receptor potential channel Vanilloid 1 (TRPV1) ion channel activation by means of the Cameleon YC3.6 calcium sensor as is reported in Chapter 3. This assay was performed with LC-MS fractions and whole extracts of chilli pepper fruits which led to the identification of new ion channel agonists. This chapter also discusses the possibility of coupling the receptomics assay directly to an LC-MS as an additional on-line bioactivity detector. The general discussion of this thesis (Chapter 7) elaborates on this topic with additional perspectives on the feasibility of coupling the two systems.

Chapter 4 provides an extensive technical characterization of the preparation and measurement of reverse transfected cell arrays using fluorescent proteins. The response of the Neurokinin 1-receptor in relation to its gene dose in reverse transfection was studied, as well as response reproducibility during repeated activations. 
These results led to a study of bitter taste receptors in relation to sensitivity-determining parameters such as sensor type and calcium buffering (Chapter 5). This chapter aimed to enhance the sensitivity and robustness of the receptor assay and showed proof of concept with bitter receptor arrays that performed in the same range as existing state-of-the-art platforms. Such bitter taste receptor arrays may be employed for future screenings of new bitter taste agonists or modulators and the identification of bitter principles in foods.

Development of software and statistical models -the linear mixed model, as presented in Chapter 6- to analyse this new type of data showed that a spot-based comparison of sequentially-tested samples yielded the most reliable data and largely eliminated inter-spot differences in signal strength. The method could also visualize receptor specific differences between samples in the presence of a simulated host cell response. A host cell response, induced by ATP, was used to show that specific bitter receptor responses from compound spikes were cumulative to the host cell response and can be retrieved from a host cell response signal by means of comparative analysis.

The general discussion (Chapter 7) critically discusses the advantages and limitations of this new micro-fluidics approach and details which additional developments are needed to advance the technology further. The receptomics technology as described in this thesis is argued to be complementary to microplate screening technologies and represents a new analytical paradigm. The microfluidics aspect and overall assay size reduction are more cost efficient and allow both a high content dynamics analysis as well as the development of novel applications such as direct identification of bioactive compounds by coupling of LC-MS to receptomics.

All in all, this thesis presents an enabling receptor screening technology that is based on new design principles. This receptomics technology offers novel applications and has potential in the bioactivity screening of crude extracts. 

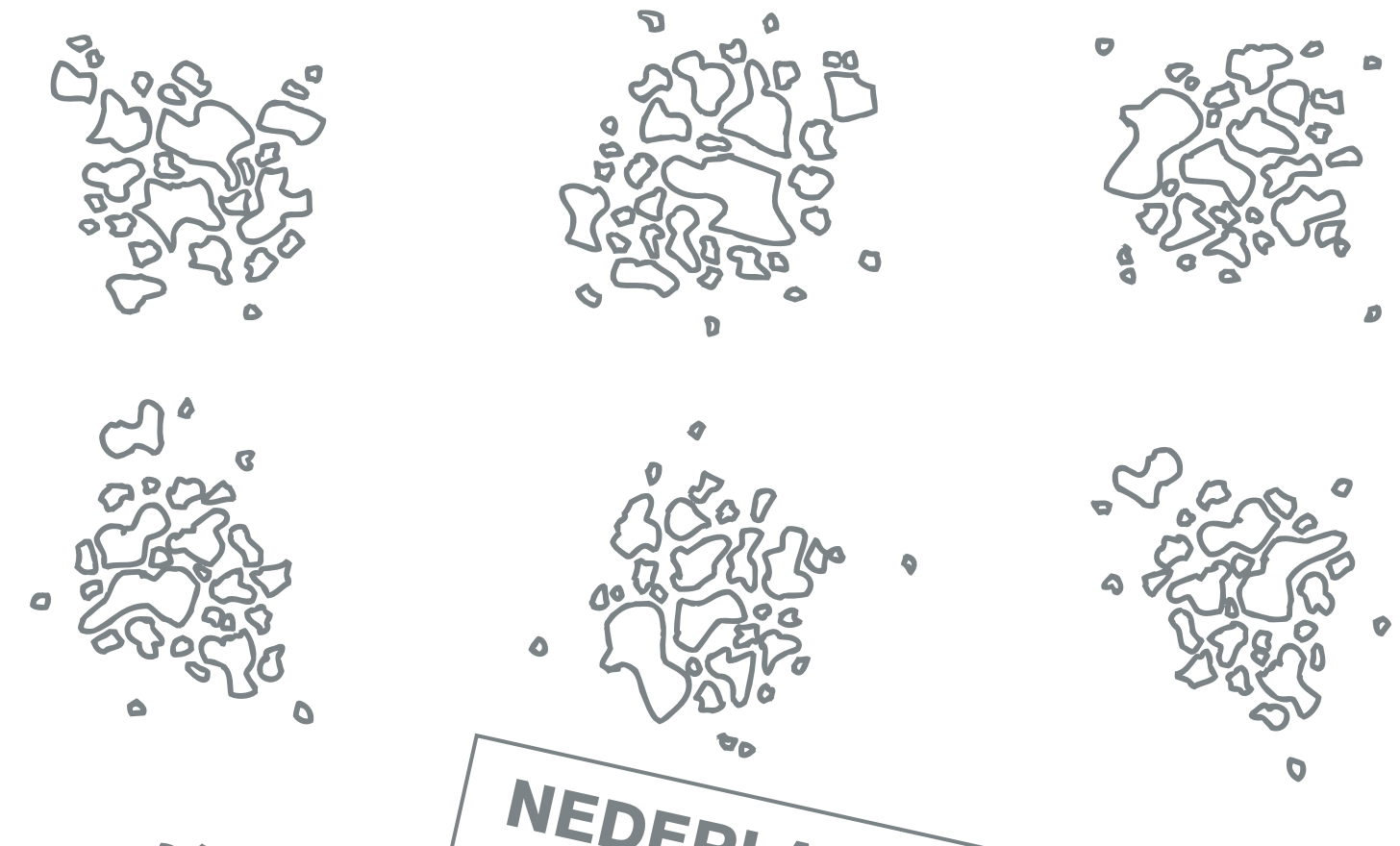

o

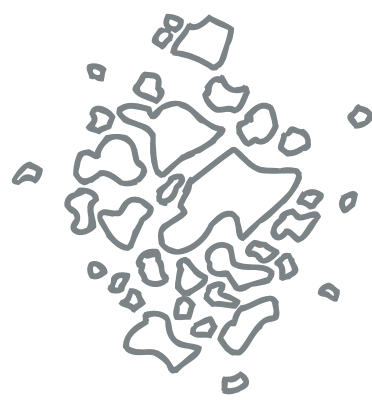

SAMENLANDSE
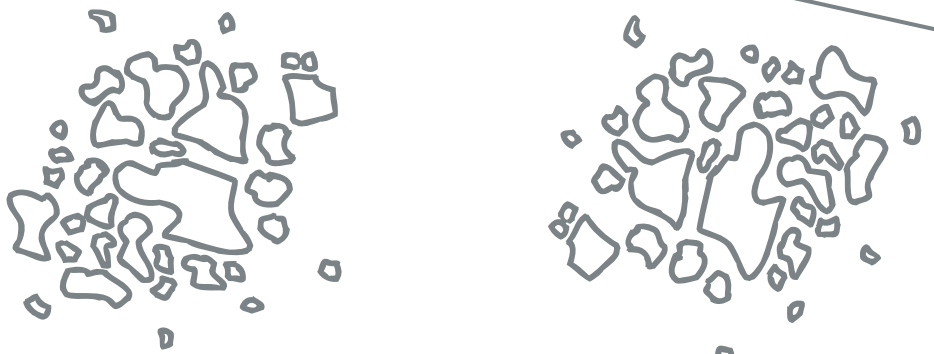

0
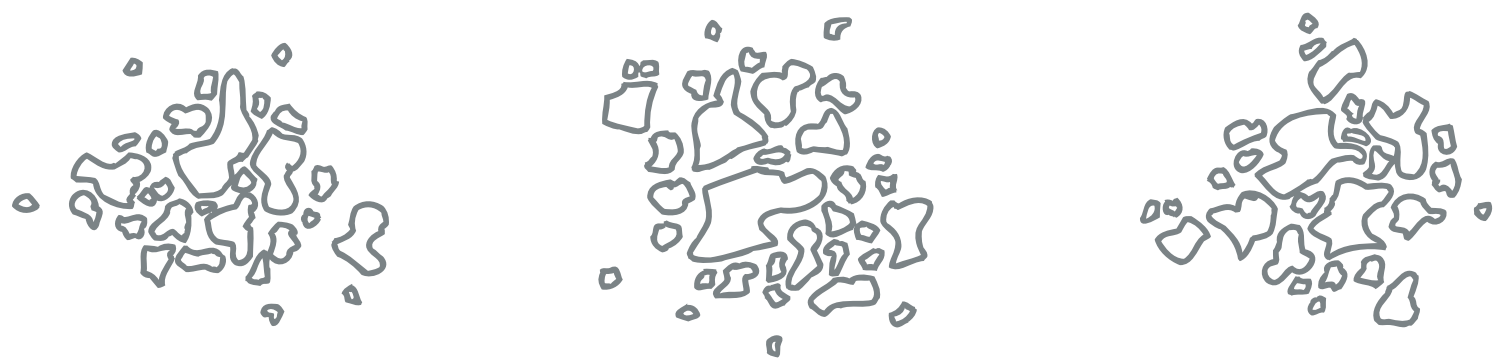
Dit proefschrift beschrijft de ontwikkeling van een technologie om $\mathrm{G}$ eiwit gekoppelde receptoren (GPCR's) te onderzoeken door middel van een gerangschikt raster (array) van receptor producerende cellen ( 300 spots) omsloten in een microfluidisch systeem. Deze technologie is ontwikkeld met het doel om smaak en/of de actieve ingrediënten in complexe monsters te bepalen. De activatie van de GPCR's wordt gemeten met behulp van een genetisch gecodeerde calcium indicator ( $\mathrm{GECI}$ ). Deze indicator is gebaseerd op het principe van FRET (Förster Resonance Energy Transfer) tussen twee fluorescente eiwitten welke gekoppeld zijn door middel van een calcium bindend domein. Wanneer deze indicator calcium bindt, dan verandert de conformatie tussen de fluoroforen. Het cel-array van verschillende receptoren is gemaakt door middel van een omgekeerde transfectie techniek waarbij geprint plasmide DNA opgenomen wordt door de cellen. De rasters worden vervolgens geassembleerd in een vloeistof kamer (flowcell), welke aangesloten wordt op een microfluidisch systeem, en geplaatst onder een stereo fluorescentie microscoop. Met deze opzet is het mogelijk om het cel-array gecontroleerd en herhaaldelijk bloot te stellen aan monsters en de cellulaire reacties door middel van de calcium indicator te meten.

GPCR's spelen een belangrijke rol in veel fysiologische en ziekte gerelateerde processen. Deze membraan eiwitten zijn geëvolueerd om een breed scala aan moleculen te herkennen, van zowel exogene of endogene oorsprong. Het signaal mechanisme is complex en heeft de potentie veel verschillende signaleringsstappen te omvatten, afhankelijk van het cel type. Het hoofdstuk "Introduction" van dit proefschrift geeft een kort overzicht van de verschillende GPCR types en hun mogelijke signaleringsstappen, met een focus op de smaak signalering. Dit hoofdstuk laat ook zien waar deze microfluidische techniek staat binnen het bestaande GPCR onderzoek veld.

Het tweede hoofdstuk onderzoekt de algemene principes, de opzet en de karakteristieken van het microfluidisch systeem om GPCR activatie te meten door middel van calcium veranderingen in HEK293 cellen. Deze cellen produceerden een combinatie van de Neurokinine-1 receptor en het Cameleon YC3.6 eiwit als calcium indicator. Er is een stabiele cel lijn gebruikt welke zorgt voor een robuuste productie met weinig variatie.

Naast GPCR's is het systeem ook gebruikt om activatie te meten van calcium ion kanaal TRPV1 met behulp van Cameleon YC3.6 zoals te lezen is in hoofdstuk 3. Deze analyse, uitgevoerd met LCMS fracties en volledige extracten van chili peper vruchten, leidde tot de ontdekking van nieuwe TRPV1 agonisten. Dit hoofdstuk bespreekt tevens de mogelijkheid om de receptomics techniek direct te koppelen aan de LCMS en te gebruiken als een gekoppelde bio-activiteit meter. De algemene discussie van dit proefschrift (hoofdstuk 7) gaat hier verder in op met verdere perspectieven over de haalbaarheid van de koppeling van deze twee systemen. 
Hoofdstuk 4 geeft een uitgebreide technische karakterisatie van de vervaardiging en de meting van omgekeerd getransfecteerde cel-arrays met gebruik van fluorescente eiwitten. Het signaal van de Neurokinine-1 receptor in relatie tot de gen dosis tijdens de transfectie is bestudeerd en daarbij ook de reproduceerbaarheid van reacties gedurende herhaaldelijke blootstellingen.

Deze resultaten leidden tot een studie naar de gevoeligheid van de bittere smaak receptoren en gevoeligheidsbepalende factoren zoals sensor type en calcium buffering (hoofdstuk 5). Het doel van dit hoofdstuk was om de gevoeligheid en robuustheid van de receptor meet techniek te verhogen en leverde een proof-of-concept dat arrays met bittere smaak receptoren kunnen presteren met vergelijkbare gevoeligheid als bestaande technieken. Deze bittere smaak receptor arrays kunnen gebruikt worden voor toekomstige onderzoeken naar nieuwe bittere smaak moleculen of modulatoren van bittere smaak en de identificatie van bitterheid voeding.

De ontwikkeling van software en statistische modellen -het lineair mixed model, zoals gepresenteerd in hoofdstuk 6- om deze nieuwe data te analyseren, toonde aan dat een analyse gebaseerd op herhaaldelijke blootstellingen van een enkele spot de meest betrouwbare gegevens opleverde en de verschillen in signaalsterkte tussen de stipjes grotendeels uitschakelde. Deze methode kon ook receptor specifieke verschillen tussen monsters aantonen in de aanwezigheid van een gesimuleerd moeder cel signaal. Een moeder cel signaal, geïnduceerd door ATP, is gebruikt om aan te tonen dat een specifieke bitter receptor signaal door toevoeging van een bittere stof een cumulatief signaal opleverde boven op het moeder cel signaal en dat deze onttrokken kon worden van het moeder signaal door middel van vergelijkend onderzoek.

De algemene discussie (hoofdstuk 7) bevat een kritische bespreking van de voordelen en beperkingen van deze nieuwe microfluidische benadering en gaat verder in op aanvullende ontwikkelingen die nodig zijn om de technologie vooruit te helpen. De receptomics technologie zoals beschreven in dit proefschrift wordt als complementair beschreven aan de bestaande receptor onderzoeks methoden en vertegenwoordigt een nieuw analytisch paradigma. Het microfluidisch aspect en algemene afname van de afmetingen van een proefopzet zijn kosten efficiënter en maken het mogelijk om zowel een hoog inhoudelijke analyse uit te voeren en nieuwe toepassingen te ontwikkelen zoals de directe identificatie van bioactieve stoffen door de koppeling van LCMS aan de receptomics.

Welbeschouwd presenteert dit proefschrift een techniek voor receptor onderzoek gebaseerd op nieuwe ontwerp principes. Deze receptomics techniek biedt nieuwe toepassingen en heeft potentieel om bio-activiteit te meten in ruwe extracten. 

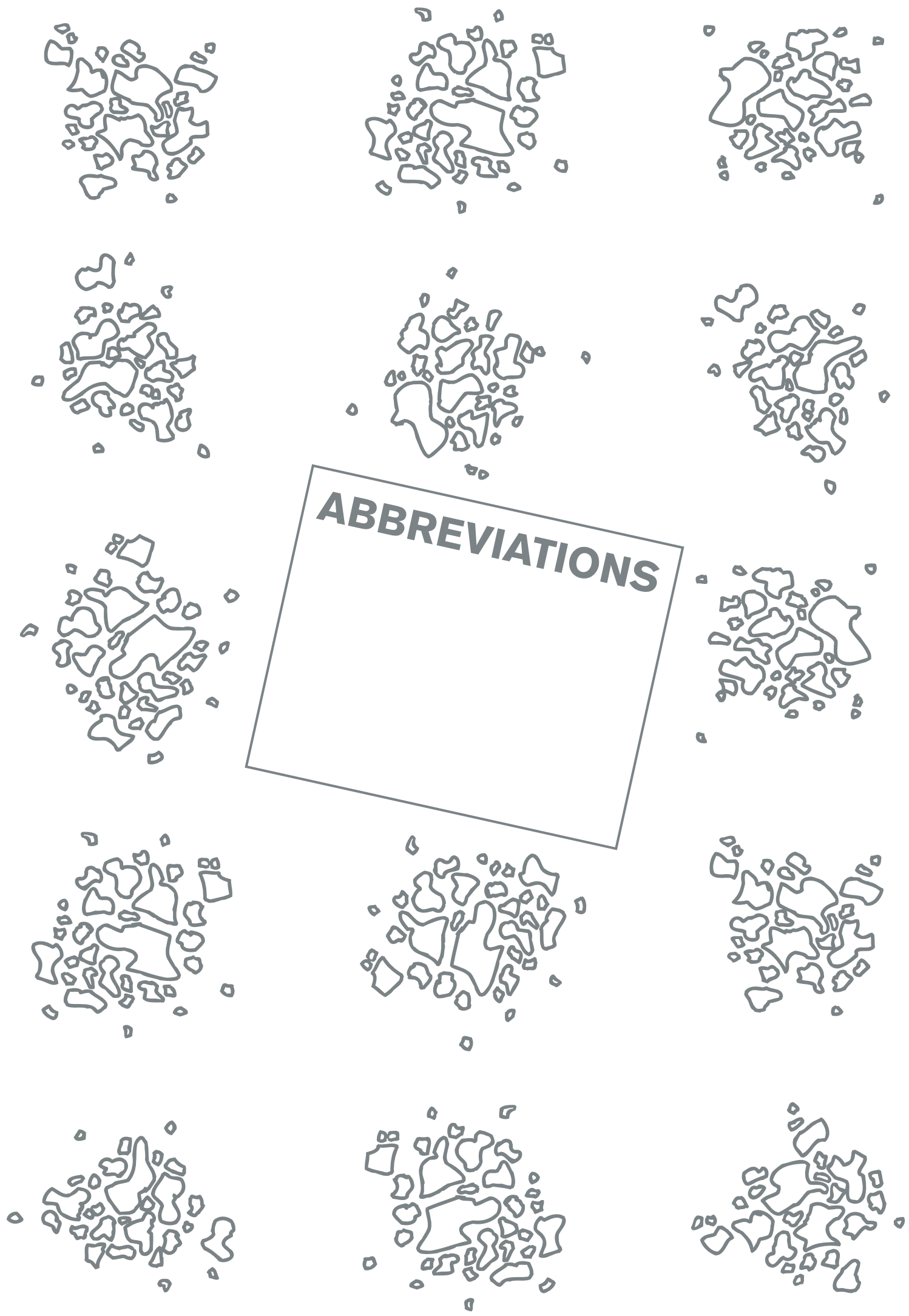


\begin{tabular}{|c|c|}
\hline$A A$ & Aristolochic acid \\
\hline$A C$ & Adenylate Cyclase \\
\hline $\mathrm{ACN}$ & Acetonitrile \\
\hline AMP & Adenosine Monophosphate \\
\hline ATP & Adenosine triphosphate \\
\hline$A U$ & Airy Unit \\
\hline BRET & Bioluminescence Resonance Energy Transfer \\
\hline CAM & Calmodulin \\
\hline CAMP & Cyclic Adenosine Monophosphate \\
\hline CFP & Cyan Fluorescent Protein \\
\hline cGMP & cyclic Guanosine Monophosphate \\
\hline $\mathrm{CHL}$ & Chloramphenicol \\
\hline CNG & Cyclic Nucleotide Gated \\
\hline CSO & Centrum voor Smaakonderzoek \\
\hline DAG & Diacylglycerol \\
\hline DB & Denatonium benzoate \\
\hline DMEM & Dulbecco's Modified Eagle Medium \\
\hline DMSO & Dimethylsulfoxide \\
\hline D-Sal & D-Salicin \\
\hline ECRO & European Chemoreception Research Organization \\
\hline EGTA & Ethylene glycol-bis(ß-aminoethyl ether)- $\mathrm{N}, \mathrm{N}, \mathrm{N}^{\prime}, \mathrm{N}^{\prime}$-tetraacetic acid) \\
\hline ER & Endoplasmic Reticulum \\
\hline FA & Formic Acid \\
\hline FBS & Foetal Bovine Serum \\
\hline FLIPR & Fluorescent Imaging Plate Reader \\
\hline FRET & Förster Resonance Energy Transfer \\
\hline FU & Fluorescence Units \\
\hline FW & Fresh Weight \\
\hline FWHM & Full Width at Half Maximum \\
\hline GDP & Guanosine Diphosphate \\
\hline $\mathrm{GECl}$ & Genetically Encoded Calcium Indicators \\
\hline GECO & Genetically-Encoded Ca2+ Indicator for Optical imaging \\
\hline GFP & Green Fluorescent Protein \\
\hline GLP-1 & Glucagon-Like Peptide 1 \\
\hline GPCR & G-Protein-Coupled Receptors \\
\hline GRK & G-Protein-Coupled Receptor Kinase \\
\hline GTP & Guanosine Triphosphate \\
\hline HEK293 & Human Embrio Kidney cells \\
\hline HPLC & High-Performance Liquid Chromatography \\
\hline HRP & Horseradish Peroxidase \\
\hline
\end{tabular}




\begin{tabular}{|c|c|}
\hline HTS & High Troughput Screening \\
\hline IMP & Inosine-5'-Monophosphate \\
\hline $\mathrm{IP}_{3}$ & Inositol1, 4, 5-Trisphosphate \\
\hline$I_{3} R$ & $\mathrm{IP}_{3}$ Receptors \\
\hline LC-MS & Liquid Chromatography-Mass Spectrometry \\
\hline LGIC & Ligand Gated Ion Channels \\
\hline MCS & Multiple Cloning Site \\
\hline MSG & Mono Sodium Glutamate \\
\hline NAM & Negative Allosteric Modulators \\
\hline NK1 & Neurokinin receptor 1 \\
\hline OR & Olfactory Receptor \\
\hline PAM & Positive Allosteric Modulators \\
\hline PCA & Principal Component Analysis \\
\hline PDA & Photodiode Array detector \\
\hline PDE & Phosphodiesterase \\
\hline $\mathrm{PIP}_{2}$ & Phosphatidylinositol 4,5-Bisphosphate \\
\hline PKA & Protein Kinase A \\
\hline PKC & Protein Kinase $\mathrm{C}$ \\
\hline PLC & Phospholipase C \\
\hline PLC $\beta 2$ & Phospholipase C Beta 2 \\
\hline PROP & 6-n-propyl-2-thiouracil \\
\hline Ptox & Picrotoxinin \\
\hline QC & Quality Control \\
\hline RFP & Red Fluorescent Protein \\
\hline RGS & Regulators of G protein Signaling \\
\hline ROI & Region Of Interest \\
\hline RT & Room Temperature \\
\hline RU & Response Units \\
\hline SNP & Single Nucleotide Polymorphisms \\
\hline SP & Substance $P$ \\
\hline T2B & Twitch2B \\
\hline TRPV1 & Transient Receptor Potential Channel Vanilloid 1 \\
\hline VFT & Venus Flytrap domain \\
\hline YC3.6 & Yellow Cameleon v. 3.6 \\
\hline YFP & Yellow Fluorescent Protein \\
\hline
\end{tabular}



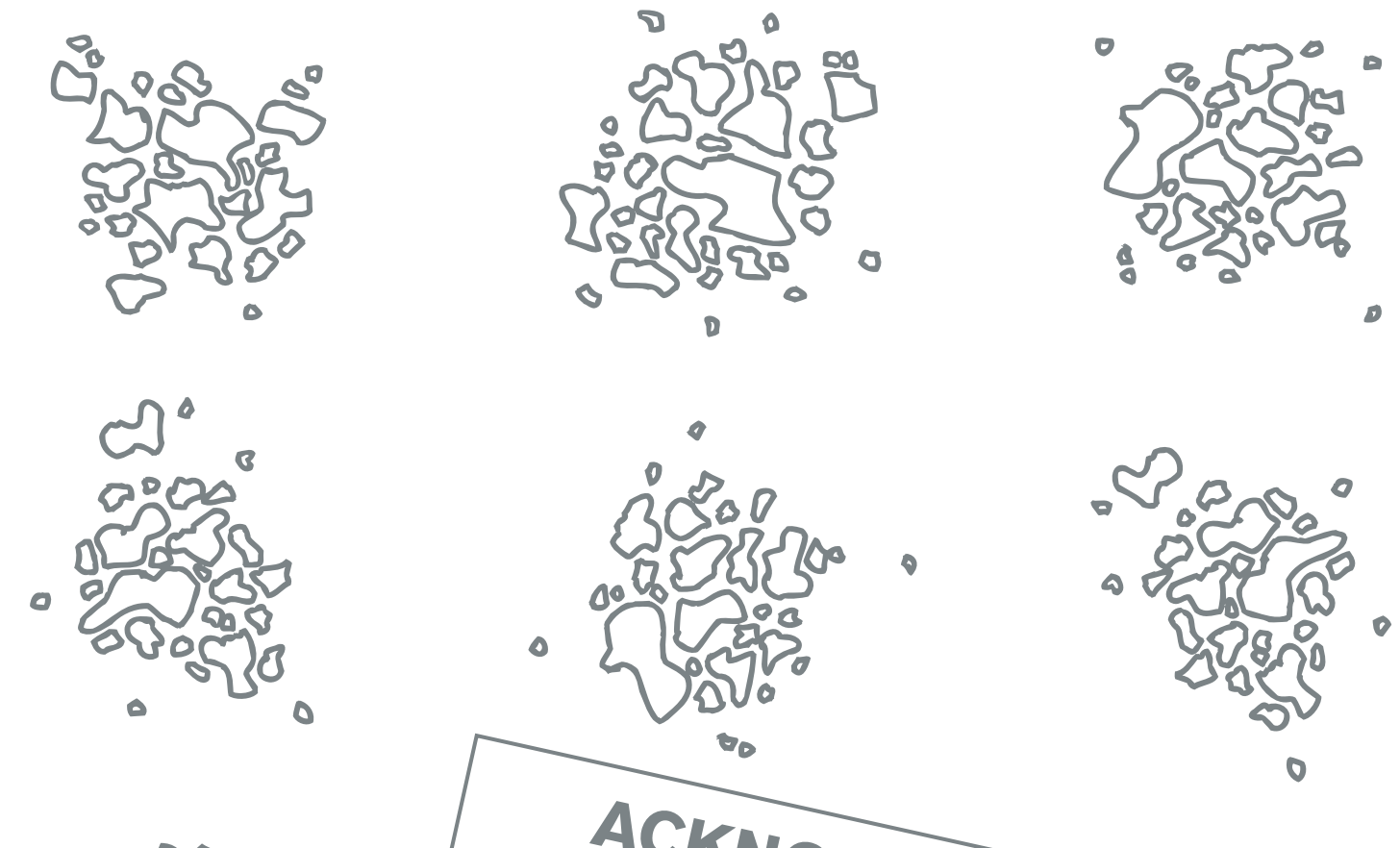

o
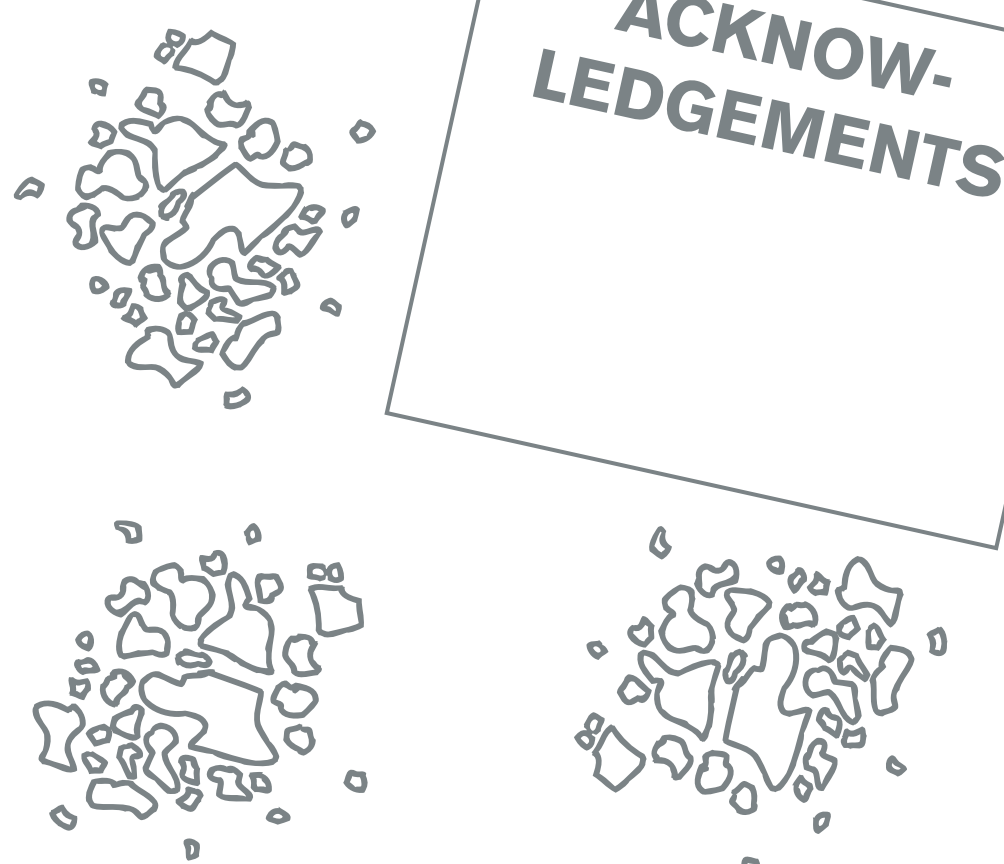

0
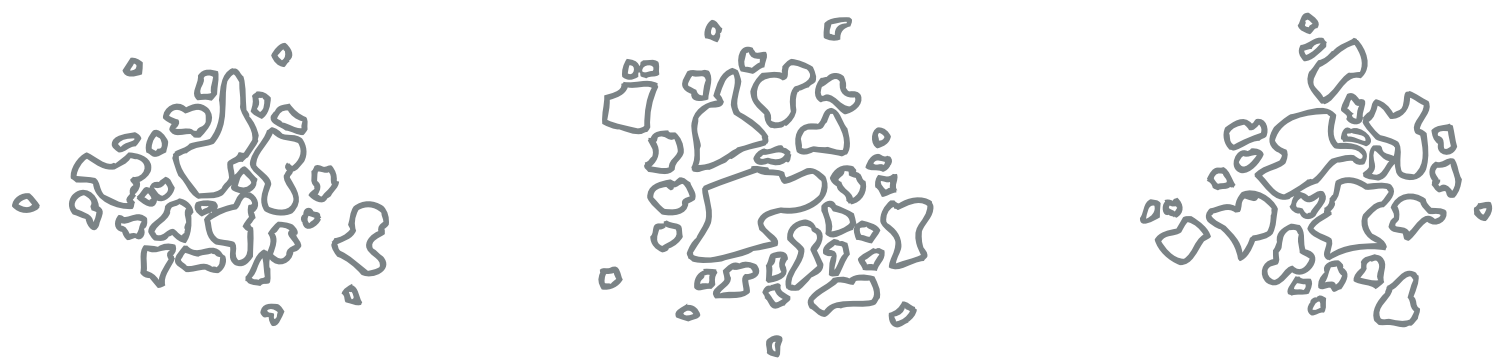
Science is a field of work that requires a lot of patience and persistence. Still we must resign ourselves to the fact that we cannot (yet) find an answer to all questions. This reminds me of a quote from my favourite astrophysicist: "The universe is under no obligation to make sense to you" (Neil deGrasse Tyson). In my efforts to make a little sense of it all, I have had the help of many colleagues, experts and friends. In this section I would like to acknowledge these people.

First I would like to thank the business unit managers of Bioscience, Ingrid van der Meer and Robert Hall, and my cluster leader Dirk Bosch for giving me the opportunity to pursue a PhD next to my position as a research assistant in the lab of Bioscience, Applied Metabolic Systems (AMS). You have shown faith in my capabilities and supported my ambitions, for which I am very grateful.

Robert Hall, as my promotor you have stood by my side and defended my position in many discussions. Keeping an eye on the goal of finishing the PhD when we were in danger of losing ourselves in many interesting side projects. You have kept a realistic view and minimized delays, thank you. Your contribution extended to your excellent writing skills as a native speaker and thereby converting many sentences from Dutch-English into English; thank you very much.

I have had the privilege of receiving support from two promotors. My second promotor being none less than Renger Witkamp from Nutritional Biology and Health, who gave me the opportunity to to add a pharmacological dimension to my project. From your former office in the old Biotechnion to the new Helix, you have inspired me with anecdotes and visions of opportunities for the receptomics technology. You have linked my work on TRPV1 work with the research projects of Jocelijn Meijerink which resulted in an interesting student project on fatty acid conjugates. Thank you for your guidance and support.

To my co-promotor, Maarten Jongsma, I like to contribute a special word of thanks. You have been both a mentor and a friend to me. Thank you for making me feel like a part of a team. Although your visionary ideas sometimes felt like "deus ex machina" events, I have enjoyed every twist and turn in the project. Besides, as the journey is more important than the destination, I feel that I have made the most interesting journey with you as my copromotor. Thank you for being my travel-guide.

To my friend, paranymph and valuable colleague, Maurice Henquet, thank you for all your team effort to develop and improve the receptomics technology. The technology would not have become what it is today without your contributions. You are co-author in almost all of my chapters, you have played a big part in shaping this thesis, thank you very much. You have often reminded me that there is more to life besides work. Your hedonic vision on 
food has led to scrumptious dialogs in the office. Thank you for your tasteful personality.

Norbert de Ruijter, my friend and paranymph, thank you for believing in me even when I had doubts whether I should commit to a PhD project. Your never-ending optimism has given me faith in a good ending. Besides your good spirit, you have also given me the advantage to profit from your microscopy expertise and cell biology knowledge. Thank you for all the inspiring talks we have had.

Harrie Verhoeven, I am grateful for your support and encouragement throughout my PhD project on both a professional and personal level. Your criticism has sparked discussions, led to revisions and resulted in a publication. I want to thank you very much for these fierce efforts. From the many conversations we have had, I remember two lessons the most: put your family at number one priority and always have a second look at your data; the data may have more stories to tell.

Leo van Griensven, you have been my guide to good cell culture practice. I have learned a lot from you in the cell culture lab, but also in the office we have shared, you have shaped my understanding of how the scientific "politics" really works. I have cherished your fatherfigure qualities and I am very grateful for the time that you have spent with us.

The receptomics bio-informatics team Ron Wehrens, Marco van Lenthe and Jasper Engel, you have done a great job of turning something difficult into routine R-scripts and intelligible software. Your efforts have been vital to the receptomics technology. Thank you very much for your valuable contributions.

My co-authors, thank you for publishing together with me. I have learned a lot discussing various aspects of the research with you. Guiding me through the writing process and reviewing the material has been a valuable experience to me. After all, I still feel that publishing is exciting and fun.

Over the years I have had the pleasure of guiding different students. Each of you helped me to develop my teaching skills and provided me with different challenges. Christa Heryanto, you were my first receptomics student and you have stayed the longest. You completed two internships and a period as a guest worker. I have enjoyed your company and cheerful attitude. I am very grateful that you have made an effort of keeping contact over the years. My hope is that, one day, you will also have a PhD behind your name. Jet Gijsbrechts, I have enjoyed your project on blood plasma receptomics. Working with human samples offered new challenges, which we learned to manage well. Emma Groot, you have done important experiments on the aforementioned TRPV1 and fatty acid conjugates. It's a pity that it's not part of this thesis, but I am still hoping for a chance to publish the results. Ana Spindler, 
you were my latest student. We learned a lot about coffee receptomics, not as easy as it may seem.

As students or guests come and go, the AMS cluster colleagues have always been there and provided me with a strong foundation of knowledge and experience. I have received help, advice, a shoulder to cry on, hands on expertise, humorous relief, support, encouragement, distractions, friendship and much, much more from these people. They deserve a place in these acknowledgments in random order: Twan, Thamara, Rumyana, Roland, Maarten, Bas, Jasper, Luud, Ric, Bert, Henriëtte, Harrie, Maurice, Jules, Katja, Adele, Dirk, Jeroen, Hanny, Hetty and Jan.

Bioscience is a great place to work! Outside of my cluster I have made warm connections with many other members of the Business Unit Bioscience and the secretary Hana NobelsSahusilawane. It would be a long list of names if I were to mention everyone. So I'd like to say a collective "thank you" to everyone for all the BU meetings, Christmas dinners, BU outings, Saint Nicolas drinks, and much more.

Dear family and friends, thank you for your interest in my work. Thank you for listening to my stories about receptors and arrays and thank you for allowing me to explain in sometimes incomprehensible words. I have the fortune of having a wide group of friends and a nursing social life involving dance evenings, shopping days, wellness visits, dinners, theatre, cinema and much more. To all my friends, thank you for being my friends and sharing your lives with me.

To my parents, Jos and Riet Roelse, I have enjoyed an unbiased upbringing in a loving environment. You have taught me valuable skills. Above and beyond the practical skills like riding a bike, you have taught me patience, kindness, compassion, endurance, perseverance, analytical thinking and a love for science fiction. My dear brother, Bas Roelse, your joy for life is an inspiration. Thank you all for being such a positive influence in my life.

Most thanks goes to Martijn Luking, for his loving support, patience and for being a devoted father and husband. Thank you for proofreading my writings, thank you for listening to my complaints and thank you for sharing my successes. I would not have started this PhD project nor would I have been able to finish it without you by my side. My children, Alice and Blake, as I try to teach you all about life, you teach me what life is all about. Thank you for bringing meaning to my life. 

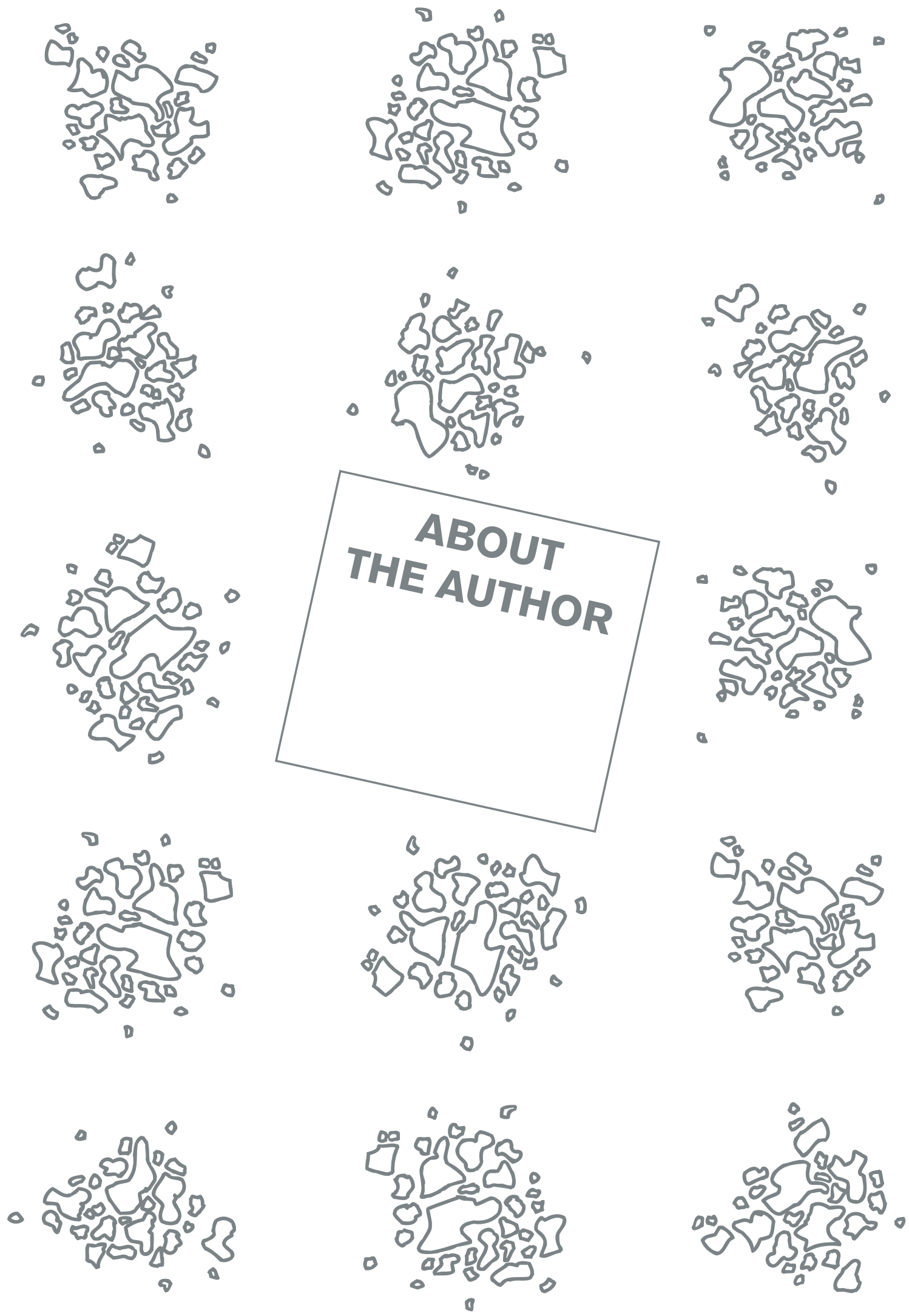
Margriet Roelse was born on November 29 1981 in Vlissingen, The Netherlands. She is married to Martijn Luking and has two children (Alice, 2010 and Blake, 2013).

Margriet graduated from high school (VWO, Scheldemond College, Vlissingen) in the year 2000 and started her bachelor study Biological and Medical laboratory research at

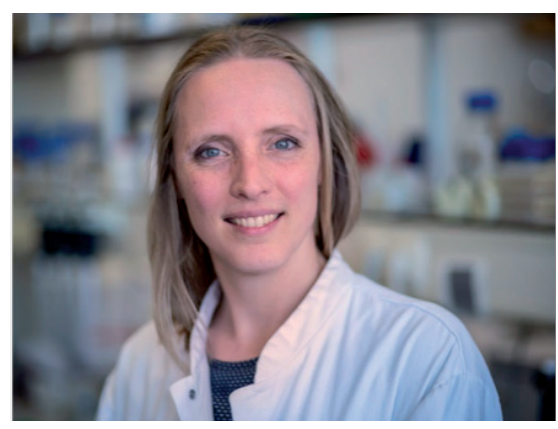
the AVANS Hogeschool in Etten-Leur, Brabant.

She completed this 4-year study in 3 years and graduated Cum Laude, after completing two internships (Erasmus MC Department of Internal Medicine and Radboud Universiteit Nijmegen Department Microbiology).

Due to her continuing interest in molecular biology she decided to follow up with a master study Molecular Life Sciences at the Wageningen University and Research. During this study she performed an internship at Catchmabs B.V. on the development of a random mutagenesis method and the application on a Llama antibody gene. And she completed her studies with a thesis project at the Laboratory of Genetics, fungal genetics group, where she studied the effect of intron alpha on the mitochondrial senescence of Podospora anserina.

After graduation as master of sciences at the Wageningen University in 2005 she was offered a job at Catchmabs B.V. as senior research assistant where she worked for 1 year as member of the product development team until the company shut down due to lack of financial investments. With her experience on Llama antibodies she next worked for one year at the University of Utrecht, department cell biology on the same topic.

Her attention was however drawn to the Wageningen University and Research department Bioscience. Where she started in 2007 as assistant to dr. Jules Beekwilder and later joined dr. Maarten Jongsma in setting up the cell culture facilities and performed preliminary research on an oligo-guided self-assembly array of cellular vesicles. This project evolved into the concept of receptomics which was awarded first prize in the PSG Business Challenge in 2012. As her experience grew within the field of receptomics, Margriet decided to write a PhD proposal which was approved in February 2015. Within the context of her PhD studies she has visited conferences to present her work, (MicroNano, NanoCity, ECRO) and participated in projects (NanoNextNL, HTP phenotyping tomato flavour) to develop the receptomics idea into the platform presented in this thesis. 

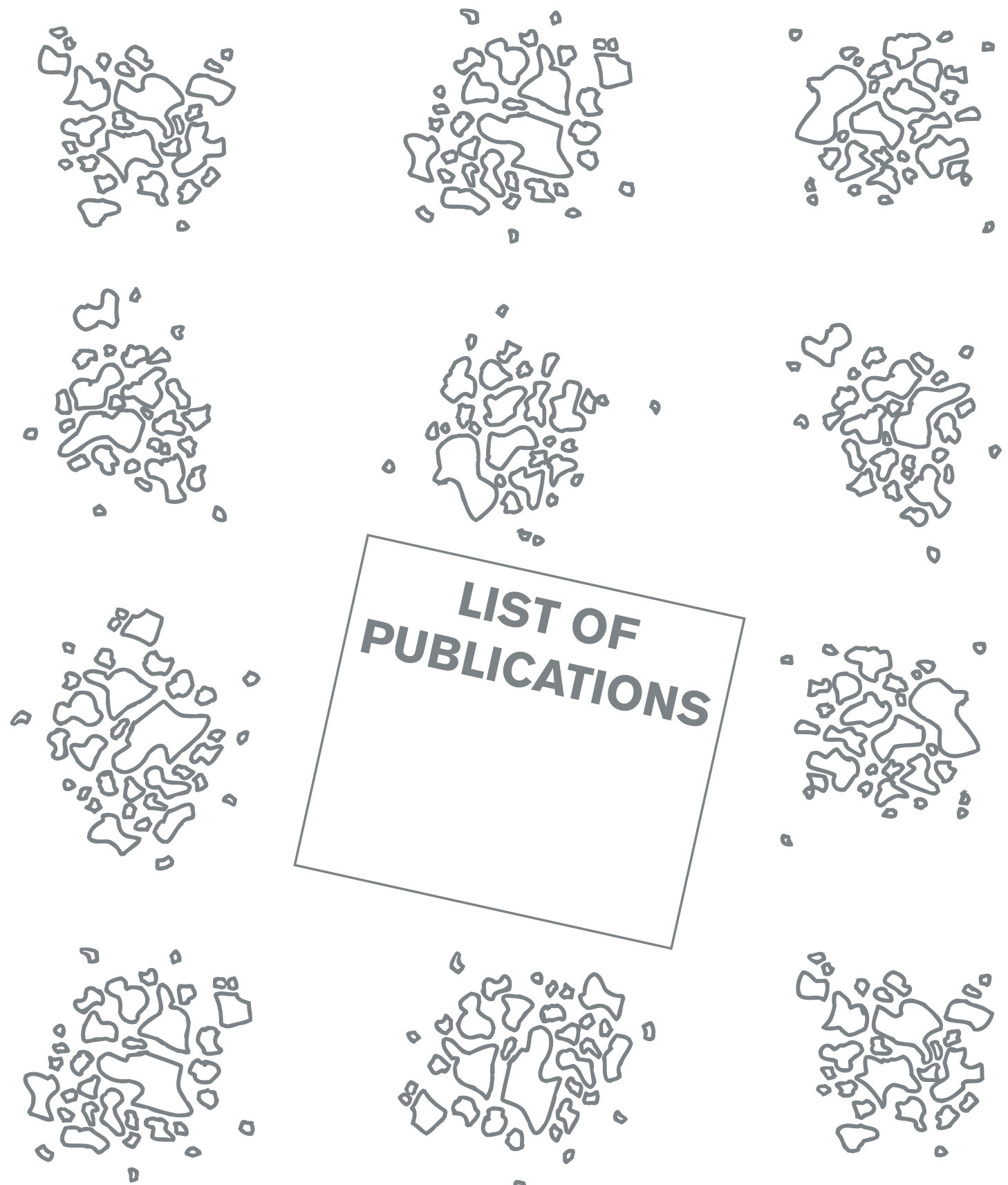

a
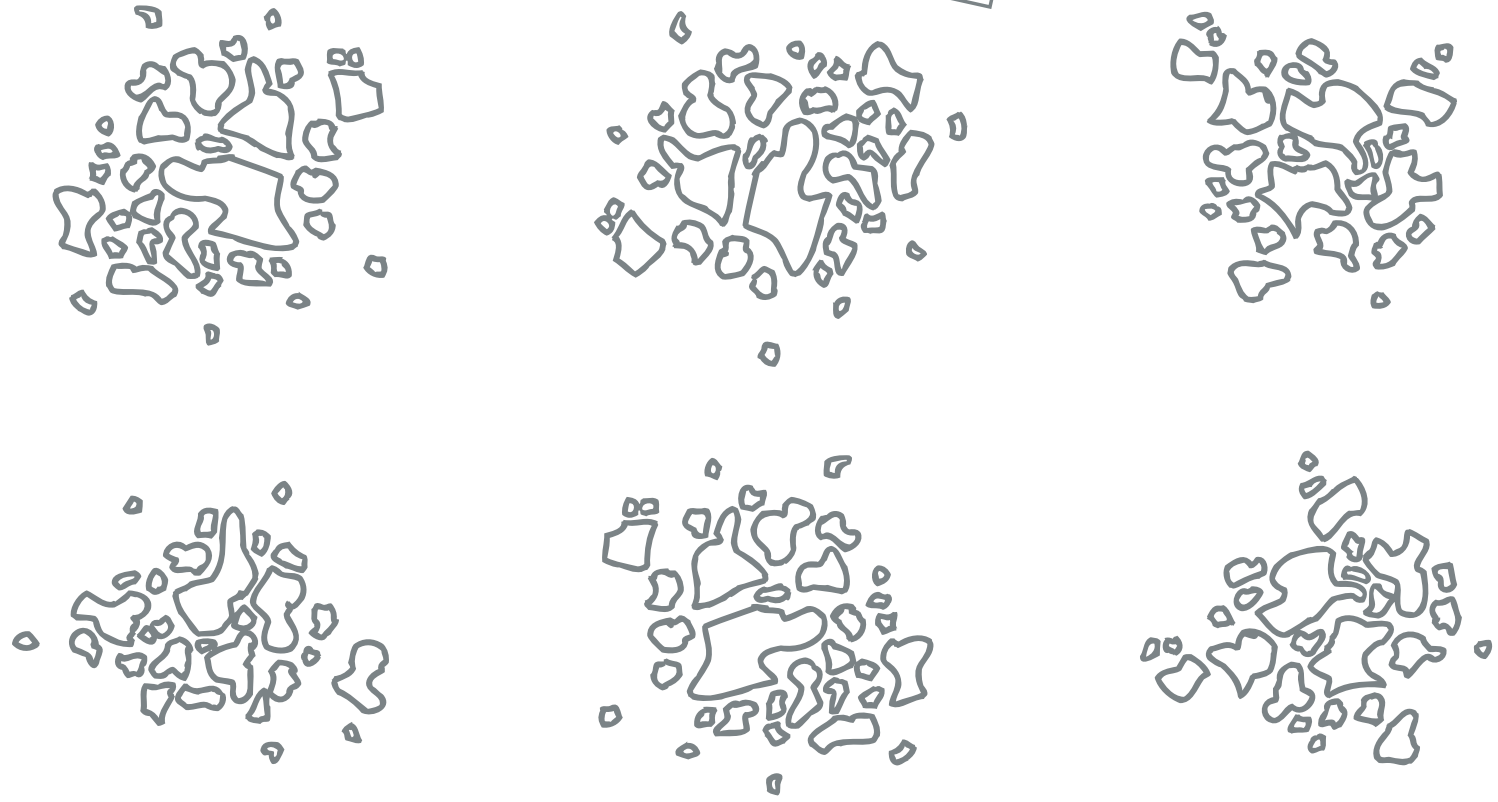
M. Roelse, N.C. de Ruijter, E.X. Vrouwe, M.A. Jongsma, A generic microfluidic biosensor of $G$ protein-coupled receptor activation-monitoring cytoplasmic $[\mathrm{Ca}(2+)]$ changes in human HEK293 cells, Biosensors \& bioelectronics, 47 (2013) 436-444.

M.G. Henquet, M. Roelse, R.C. de Vos, A. Schipper, G. Polder, N.C. de Ruijter, R.D. Hall, M.A. Jongsma, Metabolomics meets functional assays: coupling LC-MS and microfluidic cell-based receptor-ligand analyses, Metabolomics : Official journal of the Metabolomic Society, 12 (2016) 115.

M. Henquet, M. Roelse, N. de Ruijter, M. Jongsma, A generic microfluidic biosensor of G proteincoupled receptor activation, Chemical senses, 42 (2017) E33-E34.

M. Roelse, M. Henquet, R. de Vos, B. Schipper, G. Polder, N. de Ruijter, R. Hall, M. Jongsma, Functional metabolomics using a microfluidic chemoreceptor cell assay, Chemical senses, 42 (2017) E20-E20.

M. Roelse, M.G.L. Henquet, H.A. Verhoeven, N.C.A. de Ruijter, R. Wehrens, M.S. van Lenthe, R.F. Witkamp, R.D. Hall, M.A. Jongsma, Calcium Imaging of GPCR Activation Using Arrays of Reverse Transfected HEK293 Cells in a Microfluidic System, Sensors, 18 (2018).

M. Roelse, M. Henquet, M. Jongsma, Receptomics: Calcium imaging of sensory receptor cell arrays in a microfluidic system and novel applications for food screening, Chemical senses, 44 (2019) E33-E34.

R. Wehrens, M. Roelse, M. Henquet, M. van Lenthe, P.W. Goedhart, M.A. Jongsma, Statistical models discriminating between complex samples measured with microfluidic receptor-cell arrays, PloS one, 14 (2019) e0214878.

M. Roelse, R. Wehrens, M.G.L. Henquet, R.F. Witkamp, R.D. Hall, M.A. Jongsma, The effect of calcium buffering and calcium sensor type on the sensitivity of an array-based bitter receptor screening assay, Chemical senses, (2019). 
M. Van Driel, M. Koedam, C.J. Buurman, M. Roelse, H. Chiba, H.A.P. Pols, J.P.T.M. Van Leeuwen, Novel Insights into the Mechanism of Action of Vitamin D Metabolites in Bone: Evidence for 1,25-Dihydroxyvitamin D3 Synthesis by Osteoblasts and Direct Stimulation of Mineralization, Calcified Tissue Int, 74 (2004) S39-S39.

M. van Driel, M. Koedam, C.J. Buurman, M. Roelse, F. Weyts, H. Chiba, A.G. Uitterlinden, H.A. Pols, J.P. van Leeuwen, Evidence that both 1alpha,25-dihydroxyvitamin D3 and 24-hydroxylated D3 enhance human osteoblast differentiation and mineralization, J Cell Biochem, 99 (2006) 922-935.

A.K. Trilling, H. de Ronde, L. Noteboom, A. van Houwelingen, M. Roelse, S.K. Srivastava, W. Haasnoot, M.A. Jongsma, A. Kolk, H. Zuilhof, J. Beekwilder, A Broad Set of Different Llama Antibodies Specific for a 16 kDa Heat Shock Protein of Mycobacterium tuberculosis, PloS one, 6 (2011).

S.K. Srivastava, R. Ramaneti, M. Roelse, H.D. Tong, E.X. Vrouwe, A.G.M. Brinkman, L.C.P.M. de Smet, C.J.M. van Rijn, M.A. Jongsma, A generic microfluidic biosensor of G proteincoupled receptor activation - impedance measurements of reversible morphological changes of reverse transfected HEK293 cells on microelectrodes, Rsc Adv, 5 (2015) 5256352570 . 

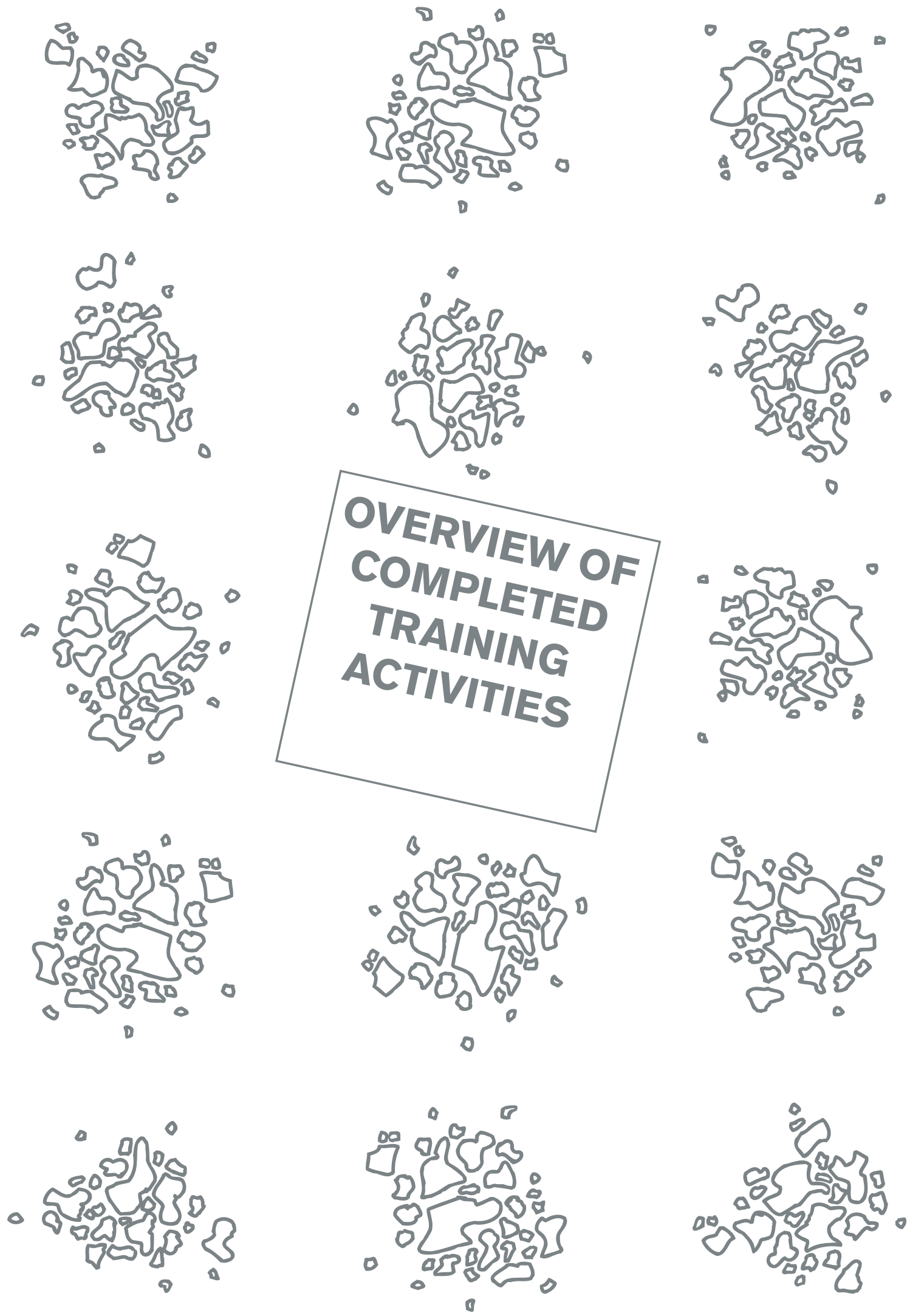
Discipline specific activities

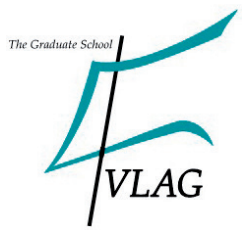

\begin{tabular}{|c|c|c|c|}
\hline Name of the course/meeting & Duration & Year & Credits \\
\hline MicroNano congress (Ede, The Netherlands) & 2 days & 2011 & 1.6 \\
\hline $\begin{array}{l}\text { Course BD Pathway microscopy system (Wageningen, } \\
\text { The Netherlands) }\end{array}$ & 3 days & 2012 & 0.9 \\
\hline MicroNano congress (Ede, The Netherlands) & 2 days & 2013 & 1.6 \\
\hline NanoCity congress (Utrecht, The Netherlands) & 2 days & 2014 & 1.6 \\
\hline RATA course and coaching (Soesterberg, The Netherlands) & 2 days & 2014 & 2 \\
\hline $\begin{array}{l}\text { Symposium From Big Data to biological solutions } \\
\text { (Wageningen, The Netherlands) }\end{array}$ & 1 day & 2015 & 0.3 \\
\hline NanoCity congress (Amersfoort, The Netherlands) & 2 days & 2015 & 1.6 \\
\hline FoodValley Expo (Ede, The Netherlands) & 1 day & 2015 & 1 \\
\hline NanoCity (Amsterdam, The Netherlands) & 1 day & 2016 & 0.3 \\
\hline ECRO congress (Athens, Greece) & 3 days & 2016 & 1.9 \\
\hline ECRO congress (Wurzburg, Germany) & 3 days & 2018 & 1.9 \\
\hline
\end{tabular}

Subtotal

\section{General courses}

\begin{tabular}{|c|c|c|c|}
\hline Name of the course & Duration & Year & Credits \\
\hline NGI venture challenge (several locations, The Netherlands) & 7 days & 2012 & 3 \\
\hline $\begin{array}{l}\text { Coaching in communication techniques by VMR (Ede, } \\
\text { The Netherlands) }\end{array}$ & n.a. & 2013 & 1 \\
\hline PhD Workshop Carousel (Wageningen, The Netherlands) & 1 day & 2015 & 0.3 \\
\hline Scientific writing (Wageningen, The Netherlands) & n.a. & 2015 & 1.8 \\
\hline Basic statistics course (Wageningen, The Netherlands) & n.a. & 2015 & 1.5 \\
\hline $\begin{array}{l}\text { Philosophy and Ethics of Food Science (Wageningen, } \\
\text { The Netherlands) }\end{array}$ & n.a. & 2018 & 1.5 \\
\hline Subto & & & 9.1 \\
\hline
\end{tabular}

\section{Optional courses and activities}

\begin{tabular}{|c|c|c|c|}
\hline Name of the course & Duration & Year & Credits \\
\hline Preparation of research proposal (Wageningen, The & & & \\
\hline Netherlands) & n.a. & 2014 & 4 \\
\hline Weekly group meetings (Wageningen, The Netherlands) & 1st - last year & n.a. & 2 \\
\hline PSG business challenge (Wageningen, The Netherlands) & 2 days & 2012 & 0.6 \\
\hline $\begin{array}{l}\text { Project meetings and presentations NanoNext NL (several } \\
\text { locations, The Netherlands) }\end{array}$ & $2014-2016$ & n.a. & 2 \\
\hline $\begin{array}{l}\text { Project meetings and presentations TKI (Wageningen, } \\
\text { The Netherlands) }\end{array}$ & $2016-2018$ & n.a. & 2 \\
\hline
\end{tabular}

Total number of credit points*

*A credit point represents a normative study load of 28 hours of study 
This work was performed at the BU Bioscience, Wageningen Plant Research and was funded by NanoNextNL, a micro- and nano-technology consortium of the government of the Netherlands and 130 partners, and Dutch Topsector Horticulture and Propagation Materials, project "HTP phenotyping tomato flavour" KV 1409-025.

Cover design by: goedinbeeld.nl

Thesis layout by: ron.nu

Printed by: proefschriftmaken.nl 

